\title{
NOVICE TEACHERS' CONCEPTIONS OF DIFFERENTIATED INSTRUCTION AND RELATED PRACTICE
}

\author{
A Dissertation \\ Presented to the Faculty of the Curry School of Education \\ University of Virginia \\ In Partial Fulfillment \\ of the Requirements for the Degree \\ Doctor of Philosophy
}

By

Hilary Dack, B.A., M.Ed., J.D.

May 2015 
(C) Copyright by Hilary Dack All Rights Reserved May 2015 


\section{EXECUTIVE SUMMARY}

\section{Dr. Carol A. Tomlinson, Advisor}

As public school classrooms in the United States grow increasingly diverse in terms of students' socio-economic status, race, and academic readiness, the expectation exists that all teachers will have the knowledge, skills, and dispositions to meet the needs of all learners in a general education setting. At the same time, state accountability systems have frequently influenced teachers to focus their efforts on teacher-centered instruction and one-size-fits-all test preparation. As a result, educational experts and professional organizations have issued calls for the personalization of curriculum and instruction to better fit the needs of individual learners. One approach to recognizing and responding to individual student differences in the general education classroom is Tomlinson's $(1999,2014)$ model of differentiated instruction.

Differentiated instruction presents a philosophy of data-driven, responsive teaching that attends to students' individual readiness, interest, learning profile, and affective needs. Although a growing body of research indicates that, when implemented with fidelity, differentiated instruction may yield significant benefits for diverse student populations, this pedagogical framework has not been implemented with fidelity in most schools, where one-size-fits-all instruction remains prevalent. The absence of the model in many American schools may be due in part to teachers' uncertainty about the nature of the model and its potential manifestations in a classroom. This uncertainty may stem from not adequately developing the knowledge, skills, and dispositions required to teach responsively as preservice teachers. No identified studies to date have examined how preservice teachers make meaning of Tomlinson's model of differentiated instruction in a 
teacher preparation course in which differentiation serves as the primary focus of instruction. Likewise, no identified studies to date have examined how novice teachers make meaning of Tomlinson's model of differentiation across both a teacher preparation course on differentiation and experiences in their early teaching careers. The present study addressed these two significant gaps in the literature.

This qualitative longitudinal multicase study examined the experiences of two participants as preservice and then first year teachers while they made meaning of differentiation as a complex philosophical approach to teaching and learning. The study occurred in two phases. Phase 1 was conducted in spring 2014 when participants were enrolled in a course on differentiating instruction taught by Tomlinson at the University of Virginia ${ }^{1}$ during their final semester of coursework. This phase explored (a) participants' conceptions of differentiation at the start of the course, (b) how those conceptions changed during the course, and (c) how factors external to the course were related to their developing conceptions. Data for Phase 1 were gathered through four one-on-one interviews spread throughout the semester; informal observations during all class meetings; and participants' course assignments, including reflections.

Phase 2 was conducted in fall 2014 after participants had graduated from their teacher preparation program and entered their own elementary classrooms. This phase explored (a) participants' conceptions of differentiation at the start of the fall, (b) how those conceptions changed during the fall, (c) the relationship between their conceptions of differentiation and their teaching practice, and (d) the relationship between contextual factors and aspects of their teaching practice related to differentiation. Data for Phase 2 were gathered through five one-on-one interviews with participants spread throughout the

\footnotetext{
${ }^{1}$ I obtained participant permission and IRB approval for the identification of the Phase 1 research site.
} 
fall; one one-on-one interview with the participant's principal or mentor; four classroom observations spread throughout the fall; and classroom artifacts. Data were collected and analyzed and conclusions were drawn through a theoretical lens informed by the situative perspective of teacher cognition and symbolic interactionism. Data analysis was conducted using the multicase study analytic approach and techniques of constant comparison supported by analytic memoing.

Key cross-case findings from Phase 1 involved the deepening of participants' complex conceptions of differentiation throughout the Differentiating Instruction course and participants' conclusions at the end of the course that differentiation would be important for their own professional success and their future students' academic success. Key cross-case findings from Phase 2 indicated that, as new teachers, both participants routinely differentiated instruction, particularly in language arts and math through modification of instruction based on student readiness, and that they recognized numerous ways in which they hoped to improve their practice related to differentiation. Participants attributed much of the knowledge, skills, and dispositions that supported their work with differentiation to their experiences in the Differentiating Instruction course. Findings suggested four assertions regarding (a) the relationship between participants' preservice coursework and their developing conceptions of differentiation, (b) characteristics of schools of employment that supported participants' implementation of differentiation as new teachers, (c) the relationship between seeing differentiation modeled and participants' conceptions of the model, and (d) the role of mindset (Dweck, 2007) in how participants made meaning of differentiation. Implications for teacher education are considered. 


\section{DEDICATION}

For

Gail H. Dack,

Christopher E. H. Dack, \&

Shannon K. Suderman

WITH APPRECIATION TO

Carol A. Tomlinson,

Catherine M. Brighton,

Nancy L. Deutsch,

Stephanie D. van Hover,

Peter Youngs, \&

my participants

iv 
TABLE OF CONTENTS

Page

LIST OF TABLES ................................................................

LIST OF FIGURES ............................................................. vii ELEMENTS

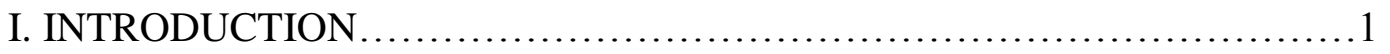

II. REVIEW OF LITERATURE........................................ 12

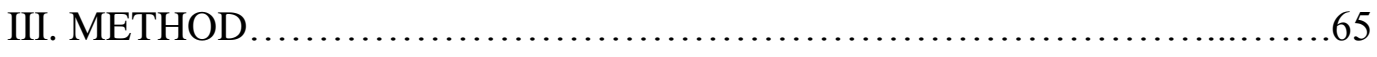

IV. FINDINGS FOR FIRST PARTICIPANT ...............................91

V. FINDINGS FOR SECOND PARTICIPANT ............................ 173

VI. CROSS-CASE THEMES, ASSERTIONS, \& IMPLICATIONS............253

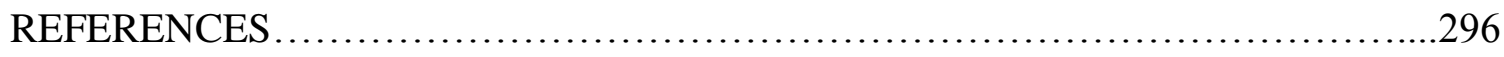

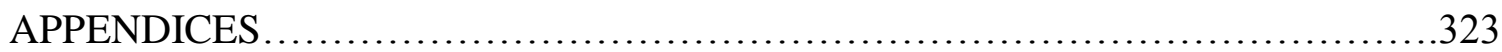




\section{LIST OF TABLES}

TABLE Page

1. Synthesis of Literature on Preservice Teachers' Beliefs and Conceptions of

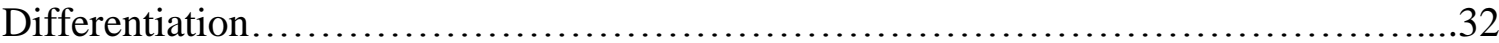

2. Synthesis of Literature on New Teacher Socialization and Implementation of Preservice Practices....................................................... 53

3. Focus Research Questions with Corresponding Data Sources......................75

4. Karen Interview Dates and Classroom Observation Dates.........................83

5. Nicole Interview Dates and Classroom Observation Dates..........................83 


\section{LIST OF FIGURES}

FIGURE Page

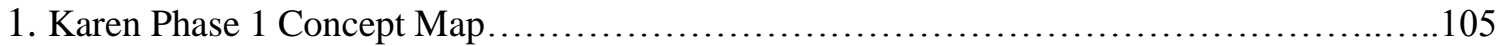

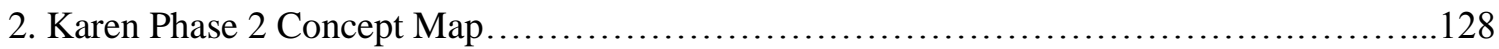

3. Nicole Phase 1 Concept Map........................................................ 188

4. Karen Phase 2 Concept Map..........................................................221 


\section{CHAPTER ONE INTRODUCTION}

Public school classrooms in the United States continue to grow increasingly diverse in terms of students' socio-economic status, race, and academic readiness (Center on Education Policy, 2007). Approximately 55 million children attend K-12 public schools, and within this population, $36 \%$ qualify for free or reduced lunch, almost $20 \%$ have parents who are immigrants, and 10\% are English language learners (Center on Education Policy, 2006). Nearly $15 \%$ of students have been identified to receive special education services, and the vast majority of these spend at least half of their day in the general education classroom (Center on Education Policy, 2006). Along with the substantial diversity represented in America's public schools, an achievement gap between Caucasian students and some minority populations persists (Berliner, 2005; Oakes, 2005). This gap has continued to serve, in part, as the impetus for educational policy reform including the No Child Left Behind Act of 2001 (NCLB; U.S. Congress, 2002). Such reforms reflect an expectation that all teachers will have the knowledge, skills, and dispositions to meet the needs of all learners in a general education setting.

Following the passage of NCLB, state accountability systems have frequently influenced teachers to focus their efforts on teacher-centered instruction, one-size-fits-all test preparation, and students who are most likely to score well on state tests if given individualized attention (Center on Education Policy, 2009; Moon, Brighton, \& Callahan, 
2003; Moon, Brighton, Jarvis, \& Hall, 2007). In response, educational experts and professional organizations have issued calls for the personalization of curriculum and instruction to better fit the needs of individual learners (National Association for the Education of Young Children, 2003; National Council for Teachers of English \& The International Reading Association, 2000; National Council of Teachers of Mathematics, 2006; Siegel \& Shaughnessy, 1994). One approach to recognizing and responding to individual student differences in the general education classroom is Tomlinson's (1999, 2014) model of differentiated instruction.

\section{Differentiated Instruction}

Differentiated instruction, or differentiation, presents a philosophy of data-driven, responsive teaching that attends to students' individual readiness, interest, learning profile, and affective needs (Tomlinson 1999, 2014). This pedagogical framework calls upon teachers to adjust curricular content, resources, instructional methods, learning activities, student products, and aspects of the learning environment in light of student differences. Its goal is to maximize the capacity of all learners by proactively designing learning experiences in response to identified needs (Tomlinson, 2005). Tomlinson (2014) identifies five interdependent principles that guide the implementation of the model, calling upon the teacher to (a) build a supportive learning environment; (b) flexibly manage the classroom; (c) develop high quality curriculum; (d) integrate ongoing formal and informal assessment to gauge student proximity to clearly articulated learning goals and to provide students with meaningful feedback on their progress; and (e) modify instruction based on learner readiness, interest, or learning profile. 
A growing body of research indicates that, when implemented with fidelity, differentiated instruction may yield significant benefits for diverse student populations (Beecher \& Sweeny, 2008; Brighton, Hertberg, Moon, Tomlinson, \& Callahan, 2005; Geisler, Hessler, Gardner, \& Lovelace, 2009; Hellman, 2007; Lou et al., 1996; Marulanda, Giraldo, \& Lopez, 2006; Reis, McCoach, Little, Muller, \& Kaniskan, 2011; Tieso, 2002; Tomlinson et al., 2003; Tomlinson, Brimijoin, \& Narvaez, 2008). Despite widespread support of differentiation, this pedagogical framework has not been implemented with fidelity in most schools, where one-size-fits-all instruction remains prevalent (Brighton et al., 2005; Kerry \& Kerry, 1997; Moon, Tomlinson, \& Callahan, 1995; Schumm et al.,1995; Stodolsky \& Grossman, 2000). The absence of the model in many American schools may be due in part to teachers' uncertainty about the nature of the model and its potential manifestations in a classroom (Santangelo \& Tomlinson, 2012). Because contemporary classrooms demand that educators, including those just entering the profession, respond effectively to the diverse needs of all students, new teachers must fully develop the knowledge, skills, and dispositions required to teach responsively upon entering the classroom.

\section{Preparing Novice Teachers}

Notwithstanding the extensive literature on learning to teach and teacher education, significant disagreement about the exact nature and extent of teacher education's influence on teacher learning persists (Glickman \& Bey, 1990; Grossman et al., 2000; Zeichner \& Tabachnick, 1981; Zeichner, Tabachnick, \& Densmore, 1987); however, the content and structure of teacher education programs have generally been found to influence preservice teachers' levels of preparation for the classroom (Darling- 
Hammond, 2006). The importance of adequate preparation of new teachers is compounded by their prevalence in American classrooms. In 2007-2008, U.S. public schools had more teachers in their first year than at any other experience level, and more than one-fifth of all teachers were in their first four years in the classroom (Carroll \& Foster, 2010).

The learning to teach is an ongoing developmental process that occurs in multiple contexts over multiple years, extending well beyond the completion of a teacher education program. These contexts include teacher education program coursework; field placements in K-12 schools; and ultimately, the teacher's own classroom in a school of employment, as well as the community, district, state, and nation in which it is located (Nolen, Horn, Ward, \& Childers, 2011). There is no guarantee that the varied contexts in which a teacher learns to teach will reflect the same or compatible philosophies of teaching and learning. The absence of this alignment may present beginning teachers with additional confusion, frustration, and other challenges beyond those typically encountered through the process of learning to teach (Liston, Whitcomb, \& Borko, 2006). New teachers' responses to these challenges carry significant implications for practice and policy, since teacher turnover costs an estimated $\$ 2.6$ billion annually (Alliance for Excellent Education, 2004).

In light of increasing student diversity in the general education classroom, the prevalence of novice teachers in American schools, and the significant costs of teacher turnover, the stakes are high when it comes to the field developing knowledge surrounding how best to prepare teachers to perform effectively in their early careers. Yet despite a widely accepted conception of learning to teach as a process of continued 
development extending from preservice education to induction and beyond (FeimanNemser, 2001), limited research has investigated novice teachers' trajectories across their preservice and early career experiences, prompting a widespread call for more longitudinal designs (Ball, 1988; Borko \& Putnam, 1996; Bryan and Atwater, 2004; Nolen et al., 2011; Thompson, Windschitl, \& Braaten, 2013; Wideen, Mayer-Smith, \& Moon, 1998). Nolen, Horn, Ward, and Childers (2011) assert: "This paucity of research makes it difficult to design professional learning programs that are responsive to the dilemmas faced by novice teachers as they negotiate meaning and form of instructional, assessment, and general pedagogical practices in new settings" (p. 89). Additionally, the limited studies that do trace the development of novice teachers' beliefs and skills across contexts and time do not adequately explain the significant variability in the teaching practice of new teachers who received the same training (Thompson et al., 2013).

\section{Preparing Novice Teachers to Differentiate Instruction}

Despite a growing need for new teachers to enter the classroom prepared to respond to academic diversity, preservice teachers typically leave preparation programs unable to do so effectively (e.g., Sands \& Barker, 2004). Some preservice teachers appear to exit programs without even recognizing a prevailing need to respond to student differences (Dee, 2011).

Many preservice teachers do leave teacher education programs with the knowledge that academic diversity exists and the belief that it warrants a response, but they lack clarity as to how to respond. For example, Tomlinson et al. (1994, 1995, 1997) conducted a study of preservice teachers at multiple schools of education involving both a control group that received no specialized training or coursework in differentiated 
instruction and treatment groups that did. In a qualitative study of only the control group, Tomlinson et al. (1994) found that participants did state beliefs in the existence and importance of differences in student needs; however, they felt that some groups of students would always be let down during instruction since addressing all student needs in one lesson was not possible. Researchers also found that these preservice teachers lacked skill in identifying academic differences, while their conceptualizations of differentiation were vague and often evident only in reactive responses to issues with individual students when one-size-fits-all planning failed (Tomlinson et al., 1994). Tomlinson et al.'s (1994) findings have been echoed by several other studies in which preservice teachers recognized academic diversity and the importance of responding to it, but reported a lack of the knowledge, skill, or dispositions needed to translate those beliefs into practice (McCray \& McHatton, 2011; Wertheim \& Leyser, 2002).

\section{Preservice Teachers' Conceptions of Differentiation}

Only three identified studies to date have examined preservice teachers' conceptions of Tomlinson's $(1999,2014)$ model of differentiation or identified factors appearing to influence student teachers' instructional practices related to differentiation (Goodnough, 2010; Tomlinson et al., 1994; Tricario \& Yendol-Hoppey, 2012).

Tomlinson et al.'s (1994) findings demonstrated preservice teachers' conceptions of differentiation when they received no training in the model, while Goodnough's (2010) contrasting findings illustrated how students' conceptions evolved over time as they engaged with the model through some explicit instruction and reflection. Tricario and Yendol-Hoppey (2012) and Tomlinson et al. (1994) identified numerous conditions that facilitated or inhibited the implementation of differentiation for student teachers. 
Goodnough's (2010) and Tricario and Yendol-Hoppey's (2012) research designs have limitations, however, and none of the three studies examined how preservice teachers make meaning of Tomlinson's model of differentiation in a teacher preparation course in which the model serves as the primary focus of instruction.

Calls have been made for teacher education programs to improve efforts to help preservice teachers acquire the knowledge, skill, and dispositions needed to address increasing diversity in the classroom through differentiated instruction (e.g., Holloway, 2000). Effectively preparing preservice teachers to differentiate as new teachers requires that well-informed instructors teach the model as a framework of "unifying principles" (Sherman, 2009, p. 57) rather than a set of strategies. Although university instructors have been called upon to integrate and model in their own teaching the philosophies and practices preservice teachers should eventually use in the field in response to academic diversity (Elksmin, 2001; Whitaker, 2011), many teacher educators do not implement a comprehensive model of differentiation in their instruction of preservice teachers (Santangelo \& Tomlinson, 2012).

\section{New Teachers' Conceptions and Implementation of Differentiation}

While both longitudinal research examining novice teachers' learning across preservice through early career experiences and research examining how preservice teachers make meaning of differentiation are scarce, longitudinal research examining new teachers' beliefs and practices involving Tomlinson's model of differentiation is almost nonexistent. Only one identified study (Tomlinson et al., 1994, 1995, 1997) with the primary goal of exploring novice teachers' beliefs and practices related to Tomlinson's 
model of differentiation across experiences in both preparation programs and teachers' early careers has been conducted to date.

Tomlinson et al.'s (1995) larger mixed methods study examined the experiences of 85 preservice teachers at six universities. None of the teacher preparation programs involved in the study required preservice teachers to take an entire course devoted to differentiation. In the first phases of the study, some preservice teachers received no treatment beyond the teacher preparation coursework presented by their universities, while others were randomly assigned to treatment groups that either participated in a oneday workshop on differentiation or participated in a one-day workshop and received curriculum coaching during student teaching (Tomlinson et al., 1994, 1995). In a subsequent phase of the study, researchers followed 10 participants from both control and treatment groups into the classroom during their first year of teaching (Tomlinson et al., 1995, 1997). Findings suggested a lack of consistency between teachers' conceptions of the model and their practices and identified a number of contextual factors that influenced differentiation in the participants' classrooms.

While Tomlinson et al.’s $(1994,1995,1997)$ findings may begin to shed light on how preservice teachers develop understandings of differentiation and how those understandings may change or be reflected in practice once inside the classroom, participants in the treatment group received only a limited introduction to differentiation during a one-day workshop lasting six hours, while researchers noted that the curriculum coaches had no notable effects on preservice teacher learning, perhaps providing "more 'noise' than clarification [in the].... already cluttered lives of novice teachers" (Tomlinson et al., 1997, p. 275). 


\section{Present Study}

No identified studies to date have examined how preservice teachers make meaning of Tomlinson's $(1999,2014)$ model of differentiated instruction in a teacher preparation course in which differentiation serves as the primary focus of instruction, allowing students to consider the model in depth. Likewise, no identified studies to date have examined how novice teachers make meaning of Tomlinson's model of differentiation across both a teacher preparation course in which differentiation serves as the primary focus of instruction and experiences in their early teaching careers. The present study addressed these two significant gaps in the literature.

\section{Research Design}

To adequately capture the complexity of teachers' conceptions of teaching and learning, qualitative methodologies must be used (Bryan \& Atwater, 2002). Bryan and Atwater (2002) note: "Research on teacher thinking has repeatedly called for understanding the interaction between teacher beliefs, actions, and the process of learning to teach, specifically through the use of multiple-year, longitudinal case studies" (p. 834). As the present study seeks to explore the interaction between novice teachers' understandings and practices as they learn to differentiate instruction, a longitudinal qualitative design spanning their preservice experiences in a teacher education program and their first year in the classroom was used. This multiphase multicase study of two participants examined their preservice experiences in a course on differentiation during spring 2014 and their in-service experiences as new teachers in fall 2014. The theories of symbolic interactionism and situative perspective informed the study's theoretical framework. 


\section{Phase 1 Research Site}

Even within teacher education programs that claim to instruct preservice teachers in differentiated instruction, teacher educators may not present Tomlinson's $(1999,2014)$ model comprehensively through their direct instruction about the model or indirect modeling of the model (Sands \& Barker, 2004; Santangelo \& Tomlinson, 2012). Rather than presenting differentiation as "representations of deeper, more fundamental philosophical orientations of worthy teaching" (Sherman, 2009, p. 44), the model may be misrepresented as a set of techniques offering a "quick fix" (Sherman, 2009, p. 51).

To ensure that preservice teachers in the study would be taught Tomlinson's $(1999,2014)$ model of differentiation comprehensively as a philosophy of teaching and learning through extensive modeling, I selected as the research site for Phase 1 a graduate-level course on differentiated instruction taught by Tomlinson at the University of Virginia. ${ }^{2}$

\section{Research Questions}

The purpose of this study was to examine how novice teachers made meaning of Tomlinson's $(1999,2014)$ model of differentiated instruction across preservice coursework specifically focused on the model and their early teaching careers. Data collection techniques including interviews, observations, and document analysis were used to answer two general qualitative questions reflecting the study's two phases and five more focused questions:

1. How do preservice teachers enrolled in a course on differentiated instruction in a teacher preparation setting make meaning of Tomlinson's $(1999,2014)$ model of differentiation?

\footnotetext{
${ }^{2}$ I obtained participant permission and IRB approval for the identification of the Phase 1 research site.
} 
(a) What are their conceptions of the model when they first enter the course, if any, and how do their conceptions change during the course, if at all?

(b) How do factors external to the course relate to their conceptions of the model?

2. How do teachers who completed a course on differentiated instruction in a teacher preparation setting make meaning of Tomlinson's $(1999,2014)$ model of differentiation during the fall of their first year in the classroom?

(a) What are their conceptions of the model as they first enter the classroom, and how do their conceptions change during the fall, if at all?

(b) What is the relationship between their conceptions of the model and their teaching practice?

(c) How do contextual factors relate to their conceptions of the model and their teaching practice?

Based on the findings, implications for teacher educators are identified. While the findings may also suggest some implications for school administrators, developing implications for that audience is not the immediate intent of the present study. Instead, its goal is to inform the field of teacher education about preparing preservice teachers to respond to diverse student needs in their future classrooms through differentiated instruction. 
CHAPTER TWO

\section{REVIEW OF LITERATURE}

The following review of literature serves as the foundation for the present study, which examined how novice teachers made meaning of Tomlinson's $(1999,2014)$ model of differentiated instruction across preservice coursework specifically focused on the model and their early teaching careers. It reviews extant research on novice teachers' beliefs and occupational socialization, as well as their application of the knowledge and skills learned in a teacher education setting to their teaching practice. It also provides an overview of the theories of symbolic interactionism and situative perspective, which inform the study's theoretical framework. It concludes by presenting the present study's research questions. This review anchors the single case findings, cross-case assertions, and implications for teacher education presented in subsequent chapters.

\section{Key Constructs Defined}

This study examined novice teachers' conceptions of differentiated instruction. Differentiation presents a philosophy of responsive teaching that attends to students' readiness, interest, learning profile, and affective needs (Tomlinson 1999, 2014). Tomlinson (2014) identifies five interdependent elements of a differentiated classroom: (a) a supportive learning environment; (b) high quality curriculum; (c) the modification of instruction based on learner readiness, interest, or learning profile; (d) ongoing formal and informal assessment; and (e) flexible classroom leadership and management. 


\section{Principles of Differentiated Instruction}

In a differentiated classroom, a teacher establishes a welcoming learning environment in which students feel comfortable working on tasks that may be different than their peers' assignments (Tomlinson, 2003). This environment is founded on the teacher's personal connections with students as individuals and a supportive classroom community. High quality curriculum that engages students anchors learning experiences in a differentiated classroom (Tomlinson \& McTighe, 2006). Curricular planning begins with identifying clear learning goals including the facts students will know (Ks), the skills students will be able to do (Ds), and the "essential understandings" (Us) (Wiggins \& McTighe, 2005) students will explore. Effective differentiated curriculum "teaches up" (Tomlinson \& Javius, 2012), or begins with a rich, rigorous learning activity and then scaffolds the greatest possible number of students to complete it successfully, rather than watering down material for lower readiness learners.

Modifying instruction based on learner needs is differentiation's core element. Instruction may be modified by adjusting the content students explore, how they process that content, or the products they develop to demonstrate their learning based on their readiness, interest, or learning profile (Tomlinson, 1999, 2014). Readiness is not synonymous with ability. It is a student's present level of mastery of facts and skills, or comprehension of an essential understanding. It is a flexible, not fixed, state that is different from lesson to lesson based on student proximity to specific learning goals (Tomlinson, 2014). Learning profile refers to individual characteristics that have an effect on students' preferred approaches to learning. Tomlinson (2014) suggested these 
traits may include gender, culture, learning styles, and “intelligence preferences," or strengths in different intelligence areas that can be developed (Sternberg, 1985). In responding to aspects of student learning profile, a teacher aims not to assign labels to students but to offer varied pathways to learning content or modes of expressing understanding.

In a differentiated classroom, ongoing assessment allows a teacher to identify students' needs, especially in terms of readiness. Instructional decisions are informed by assessment data. To do this, the teacher may identify patterns of need reflected in the data, temporarily group students based on those patterns, and create multiple versions of a learning experience in response to patterns of needs (Tomlinson \& Moon, 2013).

Last, the teacher leads the students and flexibly manages the processes of the classroom. To lead a differentiated classroom effectively, the teacher communicates her vision of how this philosophy of teaching and learning will be put into practice in her classroom and invites students to partner with her in enacting that vision (Tomlinson \& Imbeau, 2010).

\section{Mindset}

Developing the type of learning environment called for by Tomlinson's (1999, 2014) model of differentiated instruction requires the establishment of a supportive community among teacher and students informed by a growth, rather than fixed, mindset (Dweck, 2007). Through a large body of research (see Dweck, 2000, for an overview), Dweck and colleagues have identified two implicit theories of intelligence that Dweck term "mindsets" (Dweck, 2007). Those with a "growth mindset" believe that individuals have some control over their intelligence; they believe that, with studying and learning, a 
person's intelligence level can be increased (Bandura \& Dweck, 1985; Dweck, 2007; Dweck \& Leggett, 1988). In contrast, those with a "fixed mindset" believe individuals are born with a pre-determined level of intelligence dictated by genetics; they view intelligence as a static trait that cannot be changed (Bandura \& Dweck, 1985; Dweck, 2007; Dweck \& Leggett, 1988). Those with growth mindsets view mistakes as problems to be solved and opportunities for growth, while those with fixed mindsets see mistakes as threats to their intelligence or competence (Dweck, 2007).

This has significant implications for teaching and learning. If a teacher has a growth mindset, when she recognizes that a student lacks particular knowledge and skills, she does not view this as tantamount to the student's limited potential for learning. As Dweck (2000) explains, with a growth mindset, "currently low skills do not preclude future high skills" (p. 84). A teacher who views intelligence as a fixed trait, however, is likely to characterize each student as having a certain level of intelligence, which in turn dictates what that student can and cannot learn. A fixed mindset can significantly influence what and how a teacher teaches (Brown, Palincsar, \& Purcell, 1984; Howard, 1995).

As a teacher subscribes to a theory of intelligence, so too do her students. Children often learn a mindset from the attitudes of the adults with whom they interact, and which mindset a student adopts often predicts how he will respond to failure (Dweck, 1975). If a student who faces failure sees intelligence as fixed, he is likely to respond by viewing the situation as out of his control and beyond improvement (Dweck, 1975). In contrast, a student with a growth mindset who is faced with failure does not immediately give up, remaining focused on potential mastery of the task despite current obstacles 
(Diener \& Dweck, 1978, 1980). When presented with challenges, fixed mindset students have much greater difficulty than growth mindset students maintaining their confidence (Dweck, 2000). Students with growth mindsets are generally more academically motivated than their fixed mindset peers, and they tend to perform at higher levels (Blackwell, Trzesniewski, \& Dweck, 2007; Dweck, 2000). Teachers' conceptions of intelligence can influence students' beliefs about intelligence (Oakes, Wells, Jones, \& Datnow, 1997; Watanabe, 2006), which in turn can influence student achievement and motivation (Dweck, 2000).

The primary goal of a teacher in a differentiated classroom is to help all students take their next step forward in learning, recognizing that each student's entry point to learning may be at a different level of mastery, proficiency, or understanding. The belief that all students have the capability to succeed, to grow beyond where they began, is a cornerstone of the philosophy of differentiation (Tomlinson, 2014).

\section{Additional Constructs Defined}

The term "novice teachers" is defined to include both preservice teachers, the focus of Phase 1 of the present study, and teachers in the induction stage of their career, the focus of Phase 2. Novice teachers in the induction stage are referred to here as "new teachers" or "beginning teachers." These definitions are grounded in Feiman-Nemser's (2001) learning-to-teach continuum that outlines the tasks of professional learning at three stages: teacher preparation, new teacher induction, and continuing professional development. Feiman-Nemser (2001) suggests that the second stage of new teacher induction occurs during the first three years of teaching. According to this framework of professional learning, the central tasks of the teacher at each stage are different. The 
tasks of the preservice teacher include a critical examination of beliefs relating to visions of good teaching and developing an understanding of issues of diversity, while the tasks of a new teacher in the induction stage include learning the context in which they teach and designing responsive instruction (Feiman-Nemser, 2001).

The construct "conception" is operationalized to encompass knowledge and beliefs (Steele \& Widman, 1997). While some have argued that the line separating knowledge and beliefs is blurred (e.g., Fenstermacher, 1994), they are generally treated as distinct constructs in the literature (e.g., Nespor, 1987). The definition of teacher knowledge used in the present study reflects constructs developed by Shulman and colleagues (Shulman 1986, 1987; Grossman, 1990) and focuses primarily on three forms: (a) general pedagogical knowledge, which transcends a particular subject area; (b) subject matter knowledge, which reflects a teacher's understanding of the conceptual structure of a discipline and particular disciplinary topics; and (c) pedagogical content knowledge of discipline-specific strategies for representing topics to students.

In contrast, beliefs are both cognitive and affective, reflecting personal values, perspectives, and assumptions (Steele \& Widman, 1997). They are "psychologically held understandings, premises, or propositions about the world that are felt to be true" (Richardson, 1996, p. 103) and are part of a larger belief system (Nespor, 1987, Pajares, 1992). Beliefs are generally fixed. When they do change, this occurs not as a result of argument or reason, but due to a form of conversion or "gestalt shift" (Nespor, 1987, p. 321). Teachers' beliefs about teaching and learning are significant determinants of how they teach, including their approaches to curriculum, instruction, assessment, and interacting with students (Nespor, 1987; Pajares, 1992; Richardson, 1996). Compared to 
knowledge, beliefs have a greater influence on how people frame problems and are stronger predictors of behavior (Nespor, 1987).

\section{Preservice Teachers' Knowledge}

Improved understandings of how experts structure and access knowledge for performance and problem-solving (e.g., Bransford, Brown, Cocking, \& National Research Council, 1999) have led researchers to focus greater attention on the nature and organization of common teacher knowledge. Multiple systems of categorizing teacher knowledge have been suggested (e.g. Carter \& Doyle, 1987; Clandinin, 1986; Grossman, 1990; Shulman, 1986). Although it has been noted that any such categorization is somewhat arbitrary (Borko \& Putnam, 1996) and the boundaries between categories are necessarily blurred due to the interrelated nature of knowledge (Marks, 1990), classification systems nevertheless provide useful heuristic devices for considering the forms of knowledge potentially involved in learning to teach.

\section{Categories of Knowledge}

Shulman (1986) is largely credited with calling the field's attention to the development of teacher knowledge and its role in "the transition from expert student to novice teacher" (p. 8). Initially, Shulman (1986) categorized teacher knowledge into (a) subject matter content knowledge, involving the structure of the discipline's concepts and principles forming an organizational system for its facts; (b) pedagogical content knowledge, including knowledge of specific topics within a discipline and of the most powerful representations or illustrations of those topics for students; and (c) curricular knowledge, encompassing knowledge of instructional materials and connections between topics of study and other disciplinary and interdisciplinary topics. In this classification 
system, scholarship in the content disciplines is the source of subject matter content knowledge, while the other two forms of knowledge are unique to teaching. Shulman and colleagues later came to identify four categories of subject matter content knowledge: knowledge of content, including concepts, procedures, and facts; beliefs about the discipline; knowledge of substantive structures; and knowledge of syntactic structures (Grossman, 1989a).

Shulman (1987) subsequently expanded the initial, three-pronged organizational system to include four additional categories: knowledge of learners, knowledge of educational aims, knowledge of educational contexts, and general pedagogical knowledge. The author defined general pedagogical knowledge as principles and techniques of classroom organization, management, and instruction that apply to pedagogy broadly and are not limited to a given topic or discipline (Shulman, 1987).

Grossman (1990) later re-conceptualized Shulman's (1986, 1987) definition by characterizing pedagogical content knowledge to encompass its original definition of knowledge of representations and strategies for teaching particular topics, as well as subject matter content knowledge, curricular knowledge, and knowledge of students' common understandings and misconceptions about the subject area.

Additional scholars have put forth numerous other constructs that attempt to reflect the multi-faceted nature of teacher knowledge (see, e.g., Calderhead, 1996). However, categorizations based on teachers' (a) general pedagogical knowledge that transcends a particular subject area; (b) subject matter knowledge that reflects an understanding of the conceptual structure of a discipline and knowledge of particular disciplinary topics; and (c) pedagogical content knowledge of discipline-specific 
techniques of representing topics to students remain commonly used heuristics in the field (e.g., Borko \& Putnam, 1996) and will be used to organize the following review of relevant research.

\section{Research on Preservice Teachers' Knowledge Gained through Teacher Preparation}

Research on the relationship between knowledge gained from teacher preparation and new teachers' performance is limited and, to some extent, ambiguous, contributing to the heated debate about the potential influence of teacher education on teacher effectiveness (e.g., Ballou \& Podgursky, 2000; Darling-Hammond, 2000a; DarlingHammond \& Youngs, 2002; U.S. Department of Education, 2002).

Research on general pedagogical knowledge. The limited research on teacher preparation has not focused on the relationship between specific aspects of general pedagogical knowledge acquired by preservice teachers and teacher behavior or student learning. Instead, empirical studies in this area have often looked at the impact of teacher education programs as a whole by comparing certified and uncertified teachers or considering the value added by teacher education coursework (Wilson, Floden, \& FerriniMundy, 2002). Research on potential associations between teacher certification and student achievement (e.g., Darling-Hammond, 2000b; Felter, 1999; Goldhaber \& Brewer, 2000; Grossman, 1989b) has yielded inconsistent results and has not shed light on which knowledge gained during certification coursework may have influenced outcomes. Likewise, while studies comparing the potential effects of teachers with more or less education coursework versus subject area coursework on student achievement (e.g., Adams \& Krockover, 1997; Ferguson \& Womack, 1993; Gess-Newsome \& Lederman, 1993; Grossman et al., 2000; Monk, 1994; Valli \& Agostinelli, 1993) suggest some value 
is added by education coursework, these studies fail to identify which knowledge prospective teachers learned from the coursework may be influencing their teaching practice.

Research on subject matter knowledge. Teacher preparation research has not focused on evaluating the association between preservice teachers' subject area preparation and student achievement by directly assessing participants' subject matter knowledge. Instead, subject matter knowledge has been measured through proxies such as subject area course counts, self-reports on undergraduate majors, and scores on the National Teachers Examination. The nature and volume of subject matter knowledge preservice teachers gain from disciplinary courses or from methods courses that address subject area content remain unclear. Results of the limited research on novice teachers' subject matter knowledge and student achievement (e.g., Darling-Hammond, 2000b; Ferguson \& Womack, 1993; Goldhaber \& Brewer, 2000; Guyton \& Farokhi, 1987; Hawk, Coble, \& Swanson, 1985; Monk, 1994; Rowan, Chiang, \& Miller, 1997) are contradictory and have called into question the popular belief that the greater the number of subject matter courses taken by a preservice teacher, the larger the effect on student achievement. While some studies have found positive relationships between subject matter preparation level and student achievement, a threshold effect, or a level of preparation beyond which additional preparation appears to have no effect, may be present (e.g., Monk, 1994). A number of studies have also indicated that preservice teachers frequently fail to develop the deep conceptual knowledge of a discipline that would facilitate teaching beyond basic facts and skills to include problem solving and inquiry (e.g., Adams, 1998; Ball 1990a, 1990b; Borko et al., 1992; McDiarmid \& Wilson, 
1991; Simon, 1993; Stoddart, Connell, Stofflett, \& Peck, 1993; M. Wilson, 1994; S. Wilson \& Wineburg, 1988).

Research on pedagogical content knowledge. Preservice teachers' pedagogical content knowledge remains relatively undeveloped during teacher preparation experiences (Borko \& Putnam, 1996), and educational researchers have not focused much attention on examining the ways in which students may begin to construct this knowledge during teacher preparation coursework, field experiences, or schools of employment. While field experiences in particular may be powerful contexts in which preservice teachers develop pedagogical content knowledge, research on student teaching has generally explored changes in beliefs instead. Wilson, Floden, and Ferrini-Mundy (2002) argue: "Although it is important to know how teachers feel about the benefits of field experiences, attitude surveys do not answer questions about what prospective teachers actually learn" (pp. 196-197).

Limited research has examined novice teachers' instructional representations, or decisions about how to support student understanding of a topic through explanations, examples, models, analogies, or demonstrations (e.g., McDiarmid, Ball, \& Anderson, 1989), or teachers' facility with adapting representations to the needs of particular learners. Wilson, Shulman, and Richert (1987) conducted an early study of pedagogical content knowledge in novice teachers' practice. The researchers noted in particular the challenges participants faced in considering how they would communicate their personal understanding of a topic to their students through various transformations of the content. Participants appeared to develop pedagogical content knowledge as a new form of knowledge as they shifted their thinking to focus on their students' understanding rather 
than their own. Feiman-Nemser and Buchmann (1985) have described this aspect of pedagogical content knowledge as "how to build bridges between one's own thinking and that of one's students" (p. 1). Wilson et al. (1987) noted that participants' developing pedagogical content knowledge was also enriched by their general pedagogical knowledge and their knowledge of learners, educational contexts, and curriculum, underscoring the interrelated nature of forms of teacher knowledge.

While some level of general pedagogical, subject matter, and pedagogical content knowledge must be in place for any complex instructional approach to be implemented in the classroom, knowledge is not enough. Ultimately, for a multi-faceted instructional approach requiring involved effort to be put into practice over the long term, a teacher must also believe in the power of the approach. Much of the recent literature on new teachers' conceptions and implementation of ambitious teaching practices focuses on teachers' beliefs and how to change them (Feiman-Nemser, 2001), rather than the influence of existing knowledge on practice.

\section{Preservice Teachers' Beliefs}

Preservice teachers initially approach their professional education with a host of beliefs and assumptions about teaching and learning that are already well-established (Borko \& Putnam, 1996; Feiman-Nemser, 2001; Pajares, 1992; Wideen et al., 1998). Images of teaching formed during elementary and secondary school through the long “apprenticeship of observation” (Lortie, 1975, p. 61) provide an initial lens through which preservice teachers interpret ideas encountered during teacher preparation. Thus, established beliefs shape what preservice teachers are able to learn from their teacher education programs (Feiman-Nemser, 2001). 
These beliefs can mislead preservice teachers into thinking they are more knowledgeable about teaching than they actually are and make it more challenging to form new ideas (Feiman-Nemser, 2001). Frequently, these existing assumptions are incompatible with the views of teaching and learning advocated by teacher education programs. Preservice teachers' prior beliefs frequently remain implicit and serve as filters that screen out incompatible ideas they encounter in teacher preparation settings, including new instructional approaches (Borko \& Putnam, 1996; Feiman-Nemser, 2001). As such, these beliefs often function as obstacles to change by narrowing the range of ideas preservice teachers are willing to entertain (Feiman-Nemser, 2001). Because those who enter the teaching profession have usually been comfortable and successful with traditional educational models in the past, they may find it more difficult to accept conflicting models of teaching and learning (Parajes, 1992).

These prior beliefs are robust and can be highly resistant to change (Ball, 1989; Borko et al., 1992; Weinstein, 1990). Some have suggested that preservice teachers' beliefs about teaching and learning do not change significantly as a result of their teacher preparation experiences (Kagan, 1992; Tabachnick \& Zeichner, 1984). In their research review, Stofflett and Stoddart (1992) even concluded that preservice teachers do not typically adopt new beliefs, but simply become more skillful at defending the beliefs with which they entered the profession.

Yet others maintain that these prior beliefs may not be as unyielding as suggested. A growing body of research (e.g., Ensor, 2001; Steele \& Widman, 1997) has indicated that at least some preservice teachers are open to changing their views of teaching. This is more likely to occur in teacher preparation settings where a consistent philosophy of 
teaching and learning is presented to preservice teachers throughout all facets of the program (Wideen et al., 1998) and preservice teachers are repeatedly called upon to examine their own beliefs and assumptions (Feiman-Nemser \& Buchmann, 1986).

Although it may present a challenging task for researchers (Wideen et al., 1998), understanding teachers' beliefs is essential to improving practice on a broad scale (Parajes, 1992). When a teacher attempts to implement a complex pedagogical approach that may conflict with a traditional model of schooling, the nature and depth of the teacher's beliefs about the importance and efficacy of the approach are critical and must therefore be examined. Kaplan (1991) has distinguished teachers' surface beliefs from deep beliefs, or beliefs that encompass "a personal philosophy of education to which a teacher is both intellectually and affectively committed. This kind of belief would be strongly defended if challenged, not easily shaken even in the face of discorroborating [sic] evidence... [and] embedded in a...system of related beliefs" (p. 120). Significant changes in teaching practice require changes in deep, rather than surface, beliefs (Kaplan, 1991).

The power of deep beliefs and their role in teachers' personal and professional identities should not be underestimated. Rex and Nelson (2004), who have related teachers' deep beliefs to a "powerful personal commitment" (p. 1288) to teaching, noted: "Who teachers are as professionals is so intricately tied to who they are as people that to think of teaching as a job that can be performed separately from what one believes to be important is to dehumanize the role of teacher" (p. 1321). It is the "personal sense of honor" (Rex \& Nelson, 2004, p. 1320) of a teacher with a powerful personal commitment to teaching that guides his pedagogical choices in the face of conflict, constraint, or 
ambiguity. Thus, understanding teachers' deeply held beliefs is critical to understanding their implementation of a complex approach to pedagogy — or lack thereof, particularly when a teaching setting causes dissonance or uncertainty.

The role of preservice teachers' knowledge and beliefs in learning to teach is multi-faceted. Recognizing this complexity is critical for teacher educators, as Borko and Putnam (1996) describe:

On the one hand, the knowledge and beliefs that prospective...teachers hold serve as filters through which their learning takes place. It is through these existing conceptions that teachers come to understand recommended new practices. On the other hand, knowledge and beliefs themselves are critical targets of change. Because teachers' knowledge and beliefs — about teaching, about subject matter, about learners - are major determinants of what they do in the classroom, any efforts to help teachers make significant changes in their teaching practices must help them acquire new knowledge and beliefs. Thus, a teacher's knowledge and beliefs are both the objects or targets of change and important influences on change. (p. 675)

\section{Preservice Teachers' Conceptions of Differentiated Instruction}

A review of the literature yielded three qualitative studies (Goodnough, 2010;

Tomlinson et al., 1994; Tricario \& Yendol-Hoppey, 2012) that have examined preservice teachers' conceptions of Tomlinson's $(1999,2014)$ model of differentiation. The participants in studies conducted by Goodnough (2010) and Tricario and Yendol-Hoppey (2012) all received some form of explicit training in the principles of differentiated instruction, while participants in Tomlinson et al.'s (1994) case study did not. Additionally, Goodnough's (2010) study involved students enrolled in a content methods course, while Tricario and Yendol-Hoppey (2012) and Tomlinson et al. (1994) examined the experiences of preservice teachers while student teaching.

\section{How Preservice Teachers Make Meaning of the Model: Goodnough (2010)}


Goodnough (2010) examined preservice teachers' developing conceptions of differentiated instruction in an advanced, one-semester secondary science methods course. The qualitative study used the work of 32 students in the course, including journal entries and group products, and one round of interviews with four students conducted after the completion of the course as data sources.

Key findings regarding students' developing realizations about differentiation included their (a) having little to no understanding of the philosophy before the course, (b) gradually recognizing it is a framework of guiding principles for teaching and learning rather than a set of strategies, (c) exhibiting different respective levels of understanding of its key principles, (d) distinguishing it from developing and implementing individual educational plans, (e) appreciating the importance of clear learning objectives, (f) identifying different ways of responding to variance in student learning styles, interests, and challenge level while reaching the same outcomes, $(\mathrm{g})$ making connections to what they had learned in other courses about the need to accommodate differences in student "learning styles and ability levels" (p. 250), and (h) recognizing the importance of student choice in learning activities and assessments (Goodnough, 2010). Preservice teachers in the study also anticipated six factors which might challenge the implementation of strategies that support differentiation in the classroom: "the time and energy required in the planning of DI lessons, the degree and nature of the differentiation to be used with students, the knowledge of curriculum and student abilities needed to use the approach, the equitable treatment of students, the availability of curriculum supports and supplementary resources, and the need for teacher flexibility in terms of assessment and instruction” (Goodnough, 2010, p. 255). 


\section{Supportive Conditions for Differentiating in Student Teaching: Tricario and Yendol-Hoppey (2012)}

Tricario and Yendol-Hoppey (2012) conducted a case study of three student teachers participating in an alternative certification elementary education program in which "differentiation was chosen as the focus for a major thread of their professional development workshops" (p. 143). Participants attended three of these workshops, each lasting three hours, spread throughout the year (Triacario \& Yendol-Hoppey, 2012). Data were gathered through document analysis of three differentiated lesson plans and corresponding reflections, and through participant observation on three occasions when preservice teachers were observed teaching those lesson plans.

The researchers concluded that five interdependent learning conditions supported participants' efforts to differentiate instruction during student teaching: (a) establishing collegial relationships with colleagues, leading to open communication about their teaching practice and related challenges; (b) consistent classroom management; (c) facility with the process of unpacking a standard and connecting lesson objectives, activities, and assessments to it; (d) facility with planning lessons that responded to student readiness, interest, or learning style needs through varied assignments; and (e) openness to receiving feedback and applying it in future lessons (Tricario \& YendolHoppey, 2012). Findings also suggested that the student teachers who "grew most quickly in their ability to differentiate instruction seemed to naturally embrace selfregulation as part of their professional orientation" (Tricario \& Yendol-Hoppey, 2012, p. 153), with self-regulation being defined as autonomy in monitoring and regulating actions toward the goals of expanding expertise. 


\section{Untrained Confusion about Differentiation and Barriers to Implementation in Student Teaching: Tomlinson et al. (1994)}

Tomlinson et al. (1994) reported findings from the first phase of a larger study (Tomlinson et al., 1995; Tomlinson et al., 1997) exploring the experiences of preservice teachers at multiple universities, none of which required preservice teachers to take a full course devoted to differentiation. In the initial phases of the study, some preservice teachers received no treatment beyond the teacher preparation coursework offered by their universities, while others were randomly assigned to treatment groups that either participated in a one-day workshop on differentiation or participated in the one-day workshop and received curriculum coaching during student teaching (Tomlinson et al., 1995). Tomlinson et al. (1994) presented findings from a qualitative study of six preservice teachers who received no treatment during their student teaching (Tomlinson et al., 1997). Data were gathered through three classroom observations and three interviews spread throughout the student teaching experience.

The authors found that these preservice teachers who had received no specialized training in differentiated instruction still stated beliefs in the existence and importance of differences in student needs, but felt that some groups of students would always be let down during instruction since addressing all student needs in one lesson was not possible (Tomlinson et al., 1994). Participants lacked skill in identifying academic differences, often equating behavioral compliance with academic readiness, successfully completing discrete school tasks with high ability, and struggles to learn specific content with a general inability to learn. Additionally, these preservice teachers' conceptions of differentiation were vague and were often evident only in reactive responses to issues 
with individual students when one-size-fits-all planning failed. The strategies participants used to differentiate instruction while student teaching were limited, with frequent use of cooperative learning in which more advanced students often served as teachers for less advanced learners (Tomlinson et al., 1994).

Tomlinson et al. (1994) also identified four barriers to addressing academic diversity common among the student teachers: (a) classroom management; (b) lingering notions of traditional images of teacher as knowledge-dispenser; (c) a lack of understanding and use of assessment strategies; and (d) a perceived lack of support of differentiation from cooperating teachers, university supervisors, and teacher preparation programs.

\section{Conclusions}

While Tomlinson et al.'s (1994) findings demonstrate preservice teachers' conceptions of differentiation when they received no training in the model, Goodnough's (2010) contrasting findings illustrate how students' conceptions may evolve over time as they engage with the model through some explicit instruction and reflection. Between their studies, Tricario and Yendol-Hoppey (2012) and Tomlinson et al. (1994) identify numerous conditions that may facilitate or inhibit the implementation of differentiation for student teachers. These factors may be equally influential with the experiences of new teachers.

Although these studies highlight a number of components of and influences on preservice teachers' conceptions of differentiation, they also present significant limitations. Goodnough's (2010) study, which sought to examine preservice teachers' conceptions of differentiated instruction in depth as those conceptions developed over a 
semester, did not use ongoing interviews as a data source. Four of the 32 participants were interviewed just once after the conclusion of the course. Thus, the researcher could not dialogue with students to probe their thinking for shifts in their conceptions of differentiation over time as they encountered new concepts. Similarly, Tricario and Yendol-Hoppey (2012) did not formally interview students throughout their program to probe their thinking directly, which is critical to developing a rich understanding of a participant's developing conceptions of a complex philosophy.

The literature reviewed in the Preservice Teachers' Beliefs and Preservice Teachers' Conceptions of Differentiated Instruction sections of this chapter is synthesized in Table 1. Two sets of key themes are presented in the table. The first reflects general themes involving preservice teachers' beliefs germane to the present study. The second addresses preservice teachers' conceptions of differentiation. The latter group of themes reflects both how these conceptions may develop as preservice teachers consider the model over time and the kinds of challenges or supports preservice teachers may anticipate encountering or needing in their future classrooms as they consider differentiation within the contexts of their coursework or field placement experiences. 
Table 1

Synthesis of Literature on Preservice Teachers' Beliefs and Conceptions of Differentiation

Preservice Teachers' Beliefs Themes $\frac{\text { Preservice Teachers' Beliefs }}{\frac{\text { Sources Synthesized }}{2}}$

- While preservice teachers' beliefs can be highly resistant to change, change is possible as students encounter new ideas in teacher preparation settings, especially if these ideas are presented consistently and preservice teachers are repeatedly asked to Ball, 1989; Borko et al, 1992;

Ensor, 2001;

Feiman-Nemser \& Buchmann, 1986; Steele \& Widman, 1997; examine their own beliefs.

- Changes in teacher practice require changes in deep, Kaplan, 1991 not surface, beliefs.

- Researchers must consider teachers' deep beliefs

Kaplan, 1991; Parajes, 1992 to understand their implementation, or lack of implementation, of a complex pedagogical approach.

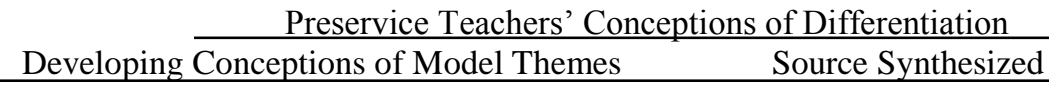

As their conceptions of the model deepen,

Goodnough (2010)

preservice teachers may:

- Correct misconceptions, such as viewing the model as a set of strategies or the equivalent of an IEP for every student.

- Make connections to topics involving the need to accommodate academic diversity studied in other courses.

- Gain knowledge of specific practices that support the model, such as the development of clear learning objectives, strategies for modifying instruction, or offering choices in assessments and activities.

- Demonstrate varied respective levels of understanding of the model's principles.

Anticipated Challenges or Necessary Supports Themes

Preservice teachers may anticipate potential challenges or supports needed when attempting to implement the model in their future classrooms:

- Time and energy for planning.

- Availability of supporting resources.

- Flexibility in instructional decision-making.

- Knowledge of content and curriculum development.

- Knowledge of strategies for instructional modifications.

- Knowledge of effective use of ongoing assessment.

- Classroom management.

- Support of colleagues and administrators.
Sources Synthesized

Goodnough (2010);

Tomlinson et al. (1994);

Tricario \& Yendol-Hoppey (2012)

\section{None of these studies examined how preservice teachers make meaning of}

Tomlinson's $(1999,2014)$ model of differentiated instruction in a teacher preparation 
course in which differentiation serves as the primary focus of instruction, allowing students to consider the philosophy in significant depth. Additionally, no identified studies to date have examined preservice teachers' conceptions of differentiation after completing general methods coursework, content methods coursework, and field experiences including student teaching, but before beginning their careers in the classroom.

\section{New Teacher Socialization}

"New teachers have two jobs - they have to teach and they have to learn to teach" (Feiman-Nemser, 2001, p. 1026). The transition from preservice preparation to practice is complex and unique (Flores, 2006). A shift in roles "from student of teaching to teacher of students" (Feiman-Nemser, 2001, p. 1026) also represents an epistemological shift, from learning about teaching through structured study to learning about teaching by encountering its daily realities. As they assume full responsibility for their own classrooms for the first time, new teachers are still learning to teach through new experiences, which are often fraught with uncertainty. Thus, this early and arguably most formative period of a teacher's development presents formidable challenges.

Beginning teachers frequently transition from teacher education programs where coursework reflects a vision of reformed instruction based on ambitious teaching practices to schools where prevailing instructional methods conflict with the innovative practices they were taught (Kennedy, 1999; Zeichner \& Tabachnick, 1981). FeimanNemser and Buchmann (1983) characterized this as the "two-worlds pitfall" (p. 19). Even when their preparation settings heavily emphasized research-based conceptual frameworks for instruction, new teachers who experience the two-worlds pitfall may feel 
great uncertainty about how to enact the ideas in the classroom or may reject them outright in favor of teacher-centered instruction (Ensor, 2001; Marbach-Ad \& McGinnis, 2009). Kennedy (1999) characterized these potential outcomes when a new teacher tries to apply what was learned in a teacher education program to a school setting with a different prevailing pedagogical approach as "problems of enactment" (p. 70). Problems of enactment require new teachers to negotiate among varied norms and messages that are often conflicting across settings (Thompson et al., 2013).

Related to problems of enactment stemming from the contradictions of two worlds is the issue of new teacher socialization. Interpretivist studies of teacher socialization reflect a conception of teachers as members of a professional culture (Brown \& Borko, 1992). Thus, the process of becoming a teacher is the process of assuming membership in a distinct culture by constructing beliefs within the culture that affect behavior. Research on teacher socialization has examined the varied forces that influence teachers as they become new participants in the culture. The process of socialization is therefore seen as a complex, "constant interplay...between individual and institutional factors" (Brown \& Borko, 1992, p. 221). Both teaching contexts and personal backgrounds influence the process of learning to teach (Flores, 2006). While older studies (e.g., Lortie, 1975) often emphasized the influence of teachers' personal backgrounds on their understandings and practices, more recent research has often focused on the effects of school culture on beginning teachers (Flores, 2006). School culture is defined as prevailing local norms relating to instruction, often communicated through interactions among teachers, administrators, students, students' guardians, mandated curriculum, and mandated assessments (McGinnis, Parker, \& Graeber, 2004). 


\section{Institutional Factors Influencing New Teacher Socialization}

There is a lack of consensus regarding the influence and potency of numerous socializing agents on new teacher development. In a seminal review of research on beginning teachers conducted between 1960 and its publication, Veenman (1984) identified the most challenging perceived problems of new teachers as classroom discipline, motivating students, addressing individual differences, assessment, relationships with parents, instructional organization, insufficient resources, and addressing individual students' problems. The author noted, however, that the findings of the reviewed studies considered neither individual teacher characteristics that may have influenced these perceptions nor participants' teaching contexts to illuminate how supportive or challenging environments influenced perceived problems (Veenman, 1984). More recent interpretive studies of new teacher socialization have also pointed to the influence of interactions between new teachers and more experienced peers within the school culture, including formal interactions with official mentors (Feiman-Nemser, 2001; Youngs, 2007; Youngs, Frank, \& Pogodzinski, 2012); professional interactions with other colleagues in the school (Coburn \& Russell, 2008; Youngs et al., 2012); and exposure to instructional norms through mentors' and colleagues' actual classroom practices (Frank et al., 2008; Youngs et al., 2012). It should be noted that, through interactions with peers, new teachers may become aware of informal subcultures within schools that conflict with the officially sanctioned practices of the formal culture (Zeichner \& Tabachnick, 1985). These conflicts between official culture and subcultures must be negotiated by new teachers. 
In their research on new teacher socialization, Zeichner and Tabachnick (1985) employed the useful heuristic of Edwards's (1979) three forms of organizational control, or formal institutional mechanisms designed to increase worker productivity and encourage adherence to prescribed norms, as a way of understanding how beginning teachers learn what the school culture expects of them, how expected behaviors are reinforced, and how members are sanctioned. The three forms are direct control, bureaucratic control, and technical control (Edwards, 1979). Direct control involves personal supervision of workers' actions by a superordinate, such as a principal, to ensure compliance to norms. With bureaucratic control, impersonal rules and hierarchical relationships embedded in the workplace social structure encourage compliance. Rewards and sanctions are dictated by official policies, rather than by a superordinate. Last, technical control is achieved through the structure of the work process, including the direction of tasks and evaluation of completed work. Higher levels of technical control minimize the need for direct and bureaucratic control to ensure compliance (Edwards, 1979; Zeichner \& Tabachnick, 1985).

In their longitudinal study of novice teachers, Zeichner and Tabachnick (1985, 1987) found direct control to be almost absent from the experiences of first year teachers, while bureaucratic control played a modest role in encouraging compliance to norms. It was technical control that served as the most powerful and prevalent form of organizational constraint in participants' socialization experiences. This control occurred through mandates involving the structure of work tasks, such as a rigid daily schedule, requirements involving the pace and format of instruction, and mandated curriculum. The authors noted, however, that the most influential determinant of all in changes in 
participants' perspectives on teaching during their first year was the interaction of the formal culture and conflicting informal subcultures.

Zeichner and Tabachnick $(1985,1987)$ did not address the potential role of peer control, or the imposition of social sanctions for teaching practices viewed as unacceptable by colleagues in non-supervisory roles. The findings presented by Zeichner and Tabachnick $(1985,1987)$ that point to the significant influence of informal subcultures created by groups of individual teachers suggest that the effects of peer approval or disapproval of new teachers' practices must also be considered.

\section{Individual Factors Influencing New Teacher Socialization}

There is also a lack of consensus surrounding the influence and potency of individual factors that may influence new teacher socialization. The body of research focused on these individual factors is smaller than that focused on institutional factors. In his seminal study of teacher socialization, Lortie (1975) maintained that new teachers drew upon models of teaching that were internalized, largely unconsciously, when they were elementary and secondary students. Thus, personal school experiences may influence how new teachers interpret and respond to their initial experiences in the classroom.

While some research has suggested beginning teachers bring to their work potential capacities and beliefs that may significantly affect their enculturation (e.g., McDonald, 1980; Sprinthall \& Thies-Sprinthall, 1983), the possible role of new teachers' personal characteristics in their socialization is not well defined in the literature. Concepts such the "powerful personal commitment" (Rex \& Nelson, 2004, p. 1288) demonstrated by experienced teachers faced with mandated change have not yet been 
applied to new teacher socialization. Zeichner and Tabachnick (1985) noted that institutional factors, such as the mechanisms of technical control, were not an "irresistible pressure for teacher conformity" (p. 17) across the board in their study; rather, new teachers were able to avoid or redirect these controls if they had "personal goals and political skills to realize these" (p. 17). Additional research is needed to determine the nature and influence of such goals and skills on new teacher socialization.

\section{Teacher Preparation's Influence on New Teacher Socialization}

In addition to institutional and individual factors, varied experiences in teacher preparation programs may influence new teacher socialization. Much has been written about the potential loss of idealism in first-year teachers as they experience a dramatic form of "reality shock" (Veenman, 1984, p. 143) in their transition from preparation to the realities of practice. New teachers typically focus on survival concerns about personal adequacy, controlling the classroom, being liked by students, and supervisor approval. An encounter with the two-worlds pitfall (Feiman-Nemser \& Buchmann, 1983) may lead to problems of enactment (Kennedy, 1999) of what was learned during teacher preparation. Many studies have examined the challenges of enactment under these conditions and the role of new teachers' beliefs about teaching and learning when challenges are encountered.

Much research conducted prior to 1980 suggested that novice teachers develop increasingly progressive attitudes towards teaching and learning during preparation and then shift to opposing traditional views when faced with the realities of the classroom during student teaching and the first years of practice (e.g., Fuller \& Brown, 1975; Zeichner, 1980). Novice teachers may become more rigid and authoritarian, with 
attitudes shifting from more idealistic to more controlling and custodial (Tomlinson et al., 1994). These early studies (e.g., Fuller \& Brown, 1975; Zeichner, 1980) seemed to reflect that teacher education programs do change the beliefs of preservice teachers, but those effects are "washed out" (Zeichner \& Tabachnick, 1981, p. 7) by everyday socialization experiences in schools through institutional factors such as principals and mentors, bureaucratic norms, and the structural characteristics of teachers' work (Zeichner, 1980).

In contrast, Lortie (1975) argued that teacher socialization is largely completed before entering a teacher preparation program through the mostly unconscious internalization of traditional teaching models as a K-12 student. According to this view, while students may appear to adopt the progressive ideologies of preparation settings, this temporary flirtation with new ideas does not outweigh lingering traditional beliefs that are too strong to be significantly changed and that lie latent until novices enter the real world of teaching. This may be compounded by the fact that preservice teachers often do not see the skills and dispositions advocated by teacher education programs as necessary or relevant (Koehler, 1985). With this argument, teacher education is seen as a feeble intervention sandwiched between personal educational experiences and teacher socialization processes (Richardson, 1996).

A third hypothesis addressing a lack of change of beliefs as a result of teacher preparation is that teacher education does in fact have a significant impact on the beliefs of preservice teachers, but that it implicitly approves traditional instructional approaches (Bartholomew, 1976). According to this view, while teacher educators may use the vocabulary of progressive teaching ideologies, they do not model progressive practices in 
their own instruction (Bartholomew, 1976). When presented with this "do as I say, not as I do" scenario, it is preservice teachers' beliefs in the efficacy of traditional approaches acquired through the apprenticeship of observation (Lortie, 1975), rather than ambitious practices, that are affirmed and reinforced.

\section{Alternative Conceptualizations of New Teacher Socialization}

Much of the literature on socialization envisions the transition from student to teacher as significantly problematic (Flores, 2006). Note Zeichner and Tabachnick (1985):

On the one hand, first-year teachers are seen as prisoners of the past (either anticipatory socialization or preservice training), and on the other hand, they are seen as prisoners of the present (institutional pressures emanating from the workplace). Significantly, in neither case are beginning teachers viewed as making any substantial contributions to the quality or strength of their own induction into teaching...[N]either of these views is very helpful in understanding beginning teacher socialization. Conformity (to the past or present) is not the only outcome of induction, and even when there is conformity, it occurs in different degrees, in different forms, and has different meanings for different individual teachers and within different institutional contexts. (pp. 3-4)

When faced with institutional pressures to change, beginning teachers may demonstrate strength and resilience, with some of their beliefs proving resistant to change (Zeichner \& Tabachnick, 1985). This is not a topic to which much focus has been directed in the empirical literature; however, Lacey (1977) proposed a theory of social strategies that does reflect the possibility of new teacher resistance.

Lacey's (1977) construct of the social strategy has been used by researchers as a heuristic device to understand how and to what degree new teachers experience socialization into their roles in a new school culture. Beginning teachers use a social strategy to make purposeful decisions when faced with institutional constraints that conflict with their beliefs (Lacey, 1977). Lacey (1977) categorizes these responses into 
three strategies of internalized adjustment, strategic compliance, and strategic redefinition. When new teachers acquiesce to an authority's interpretation of a situation or wishes, and they perceive imposed constraints to somehow be beneficial, they engage in internalized adjustment. Teachers who employ this first strategy not only willingly conform their behavior to expectations; they also make a value commitment to a new idea, representing a potential adjustment in beliefs.

The strategy of strategic compliance refers to outward compliance to institutional constraints while maintaining inner reservations about the decision (Lacey, 1977).

Teachers who strategically comply do so with the awareness that they are acting in ways that conflict with their beliefs, and their actions therefore do not represent a new value commitment.

Last, strategic redefinition involves a new teacher's attempt to change a situation that demands action that conflicts with her beliefs. In this situation, the beginning teacher does not have the formal power to make such a change, yet she manages to do so using new and creative approaches to convince those who have demanded the action to broaden the range of acceptable actions (Lacey, 1977). The attempted use of strategic redefinition may be successful or unsuccessful (Zeichner \& Tabachnick, 1985). When attempts with this strategy are unsuccessful, new teachers may respond with internalized adjustment or strategic compliance.

Lacey's (1977) theory of social strategy imbues the role of the socialized new teacher with agency. Rather than victims of insurmountable institutional pressures, beginning teachers are seen as capable of contributing to and possibly controlling their own experiences as they transition between the two worlds of preparation and practice. 


\section{Research on New Teachers' Implementation of Preservice Practices}

Limited research has investigated novice teachers' trajectories across preservice and early career experiences, prompting calls for more longitudinal designs (Ball, 1988; Borko \& Putnam, 1996; Bryan and Atwater, 2002; Nolen et al., 2011; Thompson et al., 2013; Wideen et al., 1998). The few studies that do trace the development of novice teachers' beliefs and skills across contexts do not adequately explain the substantial variability in the teaching practice of new teachers who received the same training (Thompson et al., 2013).

It appears most studies examining novice teachers' trajectories across varied learning-to-teach contexts seek to determine how and to what extent beginning teachers negotiate and implement ambitious practices learned in teacher education settings in new school of employment settings (e.g., Ensor, 2001; Flores, 2006; Grossman et al., 2000; McGinnis et al., 2004; Nolen et al., 2009; Nolen et al., 2011; Prescott \& Cavanagh, 2008; Raymond, 1997; Steele, 2001; Thompson et al., 2013).

Much of this research (e.g., Cady, Meier, \& Lubinski, 2006; Cavanagh \& Prescott, 2007; Ensor, 2001; McGinnis et al., 2004; Prescott \& Cavanagh, 2008; Raymond, 1997; Steele, 2001; Thompson et al., 2013) reflects the "macroresearch agenda in the mathematics and science education research communities [that] focuses on the links between features of teacher preparation programs and the performances of beginning teachers" (Simmons et al., 1999, p. 721), although a few studies have also looked at beginning secondary language arts teachers (e.g., Grossman et al., 2000), secondary social studies teachers (e.g., Nolen et al. 2009; Nolen et al., 2011), or teachers in a variety of subject areas (e.g., Brouwer \& Korthagen, 2005; Flores, 2006). 
A review of these longitudinal studies reveals four key themes among researchers' findings and conclusions: (a) key potential influences on new teacher practice; (b) levels and types of appropriation of concepts and practices taught in preservice settings; (c) varied awareness of inconsistencies between beliefs and practices or between practices as taught in teacher education settings and practices as enacted; and (d) improvements in teachers' readiness to implement preservice practices after the first year of teaching.

\section{Key Potential Influences on New Teachers' Uses of Preservice Practices}

Longitudinal research that examines novice teachers' trajectories across varied contexts to determine how and to what extent new teachers implement ambitious practices learned in teacher education settings points to four key potential influences on this implementation: (a) school culture as a socializing force; (b) direct connections to experiences in teacher education settings; (c) individual teachers' beliefs; and (d) individual teachers' personal traits. It should be noted that, while multiple studies identified each of these as potential influences, there is no consensus in the field as to which influences are the most powerful or widely present.

Influence of school context. In the extant literature reviewed for the present study, school context was the most commonly cited influence (Brouwer \& Korthagen, 2005; Cady et al., 2006; Cavanagh \& Prescott, 2007; Flores, 2006; Grossman et al., 2000; McGinnis et al., 2004; Nolen et al., 2011; Prescott \& Cavanagh, 2008; Raymond, 1997; Steele, 2001; Thompson et al., 2013). Brouwer and Korthagen's (2005) longitudinal mixed methods study of "how far and in what ways integrative preservice programs 
counterbalance occupational socialization" (p. 210) found socialization influences based on school context to be the most numerous and powerful of the influences studied.

The culture of the school of employment may be characterized as supportive or unsupportive of a new teacher's implementation of an ambitious practice. Numerous factors that contribute to the supportiveness of a school culture as it influences a new teacher have been identified:

- relationships with colleagues or administrators and peer support (Cady et al., 2006; Flores, 2006; Prescott \& Cavanagh, 2008; Steele, 2001);

- degree of freedom with curriculum and assessment practices (Grossman et al., 2000; Prescott \& Cavanagh, 2008; Steele, 2001);

- flexibility of time use in the face of pressures to cover content (Prescott \& Cavanagh, 2008; Raymond, 1997);

- $\quad$ teaching load (Brouwer \& Korthagen, 2005; Flores, 2006);

- availability of resources (Cady et al., 2006; Flores, 2006; Prescott \& Cavanagh, 2008; Raymond, 1997);

- classroom management issues, including discipline (Brouwer \& Korthagen, 2005; Cavanagh \& Prescott, 2007; Prescott \& Cavanagh, 2008; Raymond, 1997);

- class size (Brouwer \& Korthagen, 2005; Cady et al., 2006; Raymond, 1997); and - pupil expectations (Brouwer \& Korthagen, 2005; Prescott \& Cavanagh, 2008). Nolen et al. (2011) clarified that the "culture of practice" (p. 118) into which a new teacher is enculturated may be that of a school, but it may also be that of a particular department within a secondary school or that of an entire district. 
When contextual factors create an unsupportive school culture, new teachers' initial reactions may include: experiencing dissonance when beliefs conflict with local practice norms (Raymond, 1997); becoming skeptical about or dismissing what was learned in teacher education as impractical in the real world (Brouwer \& Korthagen, 2005; Cavanagh \& Prescott, 2007); or choosing to conform to local practice norms so as to be viewed as competent by peers (Prescott \& Cavanagh, 2008).

Connections to teacher education coursework. Fewer studies (e.g., Grossman et al., 2000; Brouwer \& Korthanger, 2005) have pointed to the direct influence of teacher education coursework on new teachers' practices. While some new teachers have reported that teacher education experiences did not significantly influence their practice (Raymond, 1997), Grossman et al. (2000) found that new teachers did use pedagogical tools taken directly from teacher education experiences in their practice, although few seemed to acknowledge this influence of preservice preparation.

In their influential mixed methods study of 350 teachers from multiple teacher preparation programs in the Netherlands, Brouwer and Korthanger (2005) concluded that teacher education can make a difference in new teachers' teaching competence. Although influences from school context were found to be more numerous and more powerful than influences from teacher education, preservice experiences influenced teaching competence as well. This influence was manifested when new teachers' preservice programs helped them see the connections between learned theory and actual classroom practice through increasingly complex student teaching activities (Brouwer \& Korthanger, 2005). 
Individual teachers' beliefs. Last, some researchers have concluded that new teachers' belief systems play a significant role in how and to what extent they implement practices and retain conceptions learned during preservice experiences (Cady et al., 2006; Raymond, 1997). Cady, Meier, and Lubinski (2006) found that new teachers who developed an "internal belief system were more likely to implement, continue to develop, and sustain practices that focus on student thinking after an initial adjustment to their new surroundings than those who did not develop them" (p. 304). The researchers conceptualized this internal belief system through Perry's (1970) construct of an internal locus of authority (Cady et al., 2006). The authors continued:

[O]nce teachers with an internal locus of authority become familiar with their school routine and their environment, they focus on improving their practices and once again reflect on the practices recommended by the teacher-education program and strive to implement these practices in their own classrooms because they have found and proven to themselves that these practices promote student learning [emphasis added]. (Cady et al., 2006, p. 304)

Raymond (1997) also concluded that beliefs played a significant role in determining practice, while finding that practice likewise influenced belief within a reciprocal relationship. Using Kaplan's (1991) constructs of deep and surface beliefs, Raymond (1997) found that, when participants' deep, traditional beliefs about mathematics content developed from their own prior educational experience conflicted with their surface, reform-based beliefs about pedagogy developed as preservice teachers, their deep beliefs won out, leading to the use of traditional practices.

Affiliation. When faced with competing messages from multiple learning-toteach contexts, teachers' personal affiliations with the sources of the messages may influence their beliefs (Ensor, 2001; Thompson et al., 2013). Thompson, Windschitl, and Braaten (2013) found that new teachers developed significant affiliations with particular 
communities' beliefs. Some affiliated chiefly with the beliefs espoused in their teacher education program, some affiliated with those of their departments in their new schools, and some maintained affiliations with beliefs presented in both areas. Similarly, Ensor (2001) characterized preservice teachers who encountered reform-based mathematics practices in a methods course as either "affiliators" who affiliated with teacher education positions and wanted to acquire and practice the pedagogical repertoire presented there, or as "selective dippers" (p. 304) who did not affiliate with teacher education positions and only acquired and practiced selected aspects of the repertoire, if any.

Motivational filters. Based on their longitudinal case-based ethnography of novice teachers, Nolen et al. (2009) proposed a theory of "motivational filters" (p. 267) to explain teachers' decisions about which ideas and practices promoted during preservice preparation to use in their own classrooms. Use of these filters appears to reflect beliefbased judgments. The four motivational filters identified by the authors were: (a) relationship filters, involving the trustworthiness or expertise of the source of information about the practice; (b) interest filters, involving connections to personal interests, such as an interest in meaningfully understanding student thinking; (c) good teacher filters, involving conceptions of good teaching practice; and (d) utility filters, involving decisions about the feasibility of the practice in a real classroom and the value of the practice for achieving a specific learning goal (Nolen et al., 2009). Similarly, though Cavanagh and Prescott (2007) did not specifically name teacher beliefs as influencing practice, one of the influencing factors they noted, new teachers' perceptions of struggling students' capacities to engage in ambitious practices, does reflect underlying beliefs about learning and ability. 
Individual teachers' personal traits. When it comes to negotiating and implementing conceptions and practices learned in teacher education, a fine line exists between teachers' beliefs and personality traits. The latter have not been addressed in this body of literature in any meaningful way, although several researchers have mentioned influential personal characteristics, sometimes almost in passing. For example, Flores (2006) noted that personal factors reflecting a "sustained commitment to teaching and learning" (p. 2047) including "intrinsic motivation to become a teacher" (p. 2047) and "commitment and willingness to 'make a difference' in students' lives" (p. 2047) influenced the practice of some new teachers studied, although these characteristics were not described further. Cavanagh and Prescott (2007) and Prescott and Cavanagh (2008) noted teachers' varied willingness to try to implement a practice a second time when not executed successfully the first time, but the authors did not name a personal characteristic that might explain this. Steele (2001) concluded that, to move beyond a traditional pedagogical approach when local norms reflected it, beginning teachers had to possess “personal commitment" (p. 160) and "professional strength" (p. 161). Similarly, Thompson et al. (2013) attributed variation in how participants negotiated competing contextual discourses, in part, to whether participants were "robust enough to reject or redirect contextual pressures to teach in conservative ways" (p. 603), though this concept of robustness in the face of competing expectations was not considered in depth. Far more research is needed to identify the potential role of personal traits as influences on novice teachers' development of conceptions and practices across contexts. 


\section{Appropriation of Concepts and Practices Taught in Preservice Settings}

A second theme emerging from studies examining how and to what extent new teachers implement conceptions and practices learned in teacher education settings within their new schools of employment involves varied forms of appropriation of concepts and practices taught in preservice settings. Thompson et al. (2013) found that beginning teachers negotiated competing contextual discourses through one of three forms of appropriation. The first, integrated appropriation, occurred when teachers implemented multiple forms of ambitious practice from their teacher education preparation, which reflected clearly defined pedagogical beliefs. Compartmentalized appropriation occurred when a lack of clearly defined pedagogical beliefs meant teachers were unable to reconcile conflicting messages from multiple contexts. These teachers might attempt to use a single ambitious practice in a lesson, but kept the practice in "conceptual isolation" (Thompson et al., 2013, p. 595) from other practices. The third form of appropriation entailed appropriating the language of ambitious practices without the actual practice. These teachers reflected traditional pedagogical beliefs and did not enact ambitious practices, although they did rename their traditional practices using reform-based vocabulary (Thompson et al., 2013). The authors attributed this variation in the nature of a participant's appropriation to factors including which community of practice's beliefs the teacher affiliated with, as discussed above, and the depth of the teacher's focus on student thinking, rather than simply the execution of strategies (Thompson et al., 2013).

The theme of new teachers appropriating reform-based vocabulary to describe traditional practices has also been reflected in other studies. For example, Ensor (2001) reported that all seven study participants claimed to use practices taught in a math 
methods course, but all had actually appropriated the vocabulary of the course and ascribed the terms new meanings to describe their own practices, which were different from those taught in coursework. The author noted that the teachers described their own traditional practices with accuracy. They had simply commandeered reform-based language to do so and seemed to genuinely believe the practices they were describing to be reform-based (Ensor, 2001). In contrast, Cavanagh and Prescott (2007) found that their participants appropriated reform language, but participants' vague use of the language suggested there was no meaningful understanding beneath their words.

\section{Awareness of Inconsistencies in Practice}

A few studies described in this section reflected that participating new teachers had varying levels of awareness of the inconsistencies between their beliefs and practices or between practices as taught in teacher education settings and practices as enacted. Flores (2006) noted that some participants in her study who reflected on their practice as new teachers were able to "identify a contradiction between what it is and what it should be.... [A]ccording to the teachers themselves, the way they taught went against the ideal beliefs they initially held" (p. 2044). This illustrates the awareness that some new teachers possess surrounding perceived inconsistencies between their beliefs or aspirations and the realities of classroom practice.

Prescott and Cavanagh (2008) reported that study participants recognized the need for the reform based practices they were taught as preservice teachers and were aware they were not enacting them, while Nolen et al. (2009) observed teachers making conscious decisions not to implement some ideas and practices learned during teacher 
preparation. In contrast, as noted above, Ensor (2001) found that all of her study

participants believed they were enacting reform-based practices when they were not.

When new teachers are aware of these inconsistences reflected in their practice or of contextual pressures that might lead to these inconsistencies, they may react by acquiescing to local norms, resisting local norms, or engaging in a form of hybrid practice that incorporates both local norms and ambitious practices (Nolen et al., 2011). If resisting local norms by defending ambitious practices or challenging traditional practices is unsuccessful, new teachers may feel compelled to change schools or districts of employment (McGinnis et al., 2004), or to leave the profession.

\section{Readiness to Implement Preservice Practices after First Year}

There is consensus in the literature that new teachers' readiness to negotiate and implement concepts and practices learned in teacher preparation improves after the first year of teaching (e.g., Brouwer \& Korthagen, 2005; Cady et al., 2006; Grossman et al., 2000). In their longitudinal mixed methods study of novice teachers, for example, Brouwer and Korthagen (2005) identified an initial "latency period" (p. 212) during the first year of teaching:

Teaching behaviors practiced during preservice programs resurfaced during the second in-service year.... The indications of a latency period suggest that program effects may take a while to manifest themselves...[D]uring this period, educational ideals had to go underground, as it were, because the beginning teachers first had to accept the leading role of the teacher and had to develop more of the instructional and educative competence they needed to put their ideals into practice. (pp. 212-213)

As a result, Grossman et al. (2000) and others have argued strongly for longitudinal research on new teachers that extends beyond the first year of practice. 


\section{Conclusions}

The literature reviewed in the New Teacher Socialization and Research on New Teachers' Implementation of Preservice Practices sections of this chapter is synthesized in Table 2. First, the socialization literature is synthesized to identify key influences that may serve as socializing agents on new teacher development. Second, the new teacher implementation literature is synthesized to identify (a) key influences that may support or inhibit implementation and (b) potential responses to those influences. This synthesis reflects the overlap among the influences on new teachers identified by the two bodies of literature. 
Table 2

Synthesis of Literature on New Teacher Socialization and New Teachers' Implementation of Preservice Practices

Influences on New Teacher Socialization

Institutional Factors

May include:

- Interactions with administrators, mentor teachers, or other colleagues (Coburn \& Russell, 2008; Feiman-Nemsor, 2001; Frank et al., 2008; McGinnis et al., 2004; Youngs, 2007; Youngs et al., 2012).

- Interactions with students and parents (McGinnis, Parker, \& Graeber, 2004).

- Exposure to instructional norms through colleagues' actual classroom practices (Frank et al., 2008; Youngs et al., 2012).

- Mandated curriculum and assessments (McGinnis et al., 2004).

May be viewed as:

- Manifestations of a particular school culture (McGinnis et al., 2004) reflecting officially sanctioned practices that may conflict with informal subcultures (Zeichner \& Tabachnick, 1985).

- Forms of direct, bureaucratic, or technical formal organizational control (Edwards, 1979; Zeichner \& Tabachnick, 1985), or informal peer control.

Individual Factors

While scarcely defined in the literature on new teacher socialization, these may include:

- Beliefs, capacities, and unconsciously internalized models of teaching (Lortie, 1975; McDonald, 1980; Sprinthall \& Thies-Sprinthall, 1983).

- Deep commitment to teaching from a particular philosophical approach (Rex \& Nelson, 2004).

- Personal teaching goals and the skills needed to achieve them (Zeichner \& Tabachnick, 1985).

Teacher Preparation

Conflicting arguments about the potential influence of teacher preparation take four positions:

- Teacher education can change preservice teachers' beliefs, but the changes are washed out by socialization (Fuller \& Brown, 1975; Zeichner, 1980).

- Teacher education does not significantly change the beliefs of preservice teachers, as they enter the program with deeply-held, though possibly unconscious, beliefs in the efficacy of traditional approaches (Koehler, 1985; Lortie, 1975; Richardson, 1996).

- Teacher education influences preservice teachers' beliefs, but it does so by implicitly approving traditional approaches through the instruction teacher educators model (Bartholomew, 1976).

- Teacher education can change preservice teachers' beliefs, and some new teachers can resist pressures of socialization to use traditional approaches (Zeichner \& Tabachnick, 1985). Resistance may occur through the use of the social strategies of strategic compliance or strategic redefinition (Lacey, 1977). 
Influences on New Teachers' Implementation of Preservice Practices

School Context Influences

May include:

- Relationships with colleagues or administrators and peer support (Cady et al., 2006; Flores, 2006; Prescott \& Cavanagh, 2008; Steele, 2001).

- Degree of freedom with curriculum and assessment practices (Grossman et al., 2000; Prescott \& Cavanagh, 2008; Steele, 2001).

- Flexibility of time use in the face of pressures to cover content (Prescott \& Cavanagh, 2008; Raymond, 1997).

- Teaching load (Brouwer \& Korthagen, 2005; Flores, 2006).

- Availability of resources (Cady et al., 2006; Flores, 2006; Prescott \& Cavanagh, 2008; Raymond, 1997).

- Classroom management issues, including discipline (Brouwer \& Korthagen, 2005; Cavanagh \& Prescott, 2007; Prescott \& Cavanagh, 2008; Raymond, 1997).

- Class size (Brouwer \& Korthagen, 2005; Cady et al., 2006; Raymond, 1997).

- Pupil expectations (Brouwer \& Korthagen, 2005; Prescott \& Cavanagh, 2008).

Influence of Individual Teachers' Beliefs and Personal Traits

When faced with competing messages from multiple learning-to-teach contexts, these influences may:

- Reflect new teachers' personal affiliations with the beliefs of particular communities, such as the teacher education program, departments within their new schools, or both (Ensor, 2001; Thompson et al., 2013).

- Prompt new teachers to use motivational filters to decide which ideas and practices promoted during preparation to use in their own classrooms (Nolen et al., 2009).

Teacher Education Coursework Influences

While limited research has examined the direct influence of teacher education on new teachers' practice:

- Teacher education has been shown to influence new teachers' teaching competence when preservice programs demonstrated connections between theory and actual practice through increasingly complex student teaching activities (Brouwer \& Korthagen, 2005).

New Teachers' Potential Responses to Influences on the Implementation of Preservice Practices May include:

- Experiencing dissonance when beliefs conflict with local practice norms (Raymond, 1997).

- Becoming skeptical about or dismissing what was learned in teacher education as impractical in the real world (Brouwer \& Korthagen, 2005; Cavanagh \& Prescott, 2007).

- Choosing to conform to local practice norms so as to be viewed as competent by peers (Prescott \& Cavanagh, 2008), while possibly appropriating the language of ambitious practices without the actual practice (Thompson et al., 2013).

- Engaging in compartmentalized appropriation by using an occasional ambitious practice but keeping it in conceptual isolation from traditional practices (Thompson et al., 2013).

- Developing an awareness of the inconsistencies between their beliefs and practices (Flores, 2006; Prescott \& Cavanaugh, 2008).

- Engaging in integrated appropriation by maintaining preservice pedagogical beliefs and implementing multiple forms of ambitious practices (Thompson et al., 2013).

- Developing improved readiness to implement ambitious practices after the first year of teaching (Brouwer \& Korthagen, 2005; Cady et al., 2006; Grossman et al., 2000). 


\section{Research on New Teachers' Implementation of Differentiation}

A review of the extant literature yielded only one study (Tomlinson et al., 1994, 1995, 1997) with the primary goal of exploring novice teachers' beliefs and practices related to Tomlinson's $(1999,2014)$ model of differentiation across experiences in both preparation programs and teachers' early careers. Two additional recent studies (Bianchini \& Brenner, 2009; Griffin \& Warden, 2006) considered new teachers' beliefs and practices related to Tomlinson's model of differentiation as one of several study foci.

\section{Differentiation and an Inclusive Culture: Griffin and Warden (2006)}

Griffin and Warden (2006) sought to evaluate a partnership between a university and public school system designed to teach preservice teachers in a special education program how to collaborate with general education teachers. Differentiated instruction was the third of three pedagogical skills emphasized in the program's coursework and fieldwork, the other skills being collaboration and cooperative teaching. When researchers surveyed recent graduates of the program who were teaching in schools of employment, they found that the most variance in predicting new teachers' use of the skills taught in the program was accounted for by the nature of the school culture in which they taught (Griffin \& Warden, 2006). Cultures that were the most supportive of inclusive models included "opportunities for heterogeneous and flexible learning opportunities with differentiated instruction occurring” (Griffin \& Warden, 2006, p. 190), while barriers to inclusive cultures included general education teachers' different beliefs and values, the absence of administrative support, and the wide range of needs represented in special educators' caseloads (Griffin \& Warden, 2006). While the use of differentiated instruction did not appear to be a major focus of the researchers' survey, their findings do suggest a connection between school culture and teachers' efforts to 
meet diverse academic needs, as well as a connection between teachers' beliefs and values and these efforts.

\section{Learning Differentiation through an Induction Program: Bianchini and Brenner}

(2009)

Bianchini and Brenner's (2009) case study of two secondary math and science teachers who participated in a state-mandated induction program during their first and second years of teaching examined the teachers' use of four "equitable educational practices" (p. 173) emphasized in the program. These practices were connecting instruction to students' experiences, instruction for English language learners, differentiated instruction, and reform-based practice (Bianchini \& Brenner, 2009). The researchers found participants' attitudes towards and attempts to use differentiated instruction reflected school context. The participant whose school context was supportive of differentiation, where her mentor and colleagues helped her to plan for and implement the model, both believed differentiation to be important for achieving equity and responding to diversity and attempted to use it. The participant whose school context was unsupportive of differentiation, where her mentor did not encourage its use, did not attempt to differentiate, deciding that the ideal of meeting individual student needs that she brought from teacher education was not feasible when she "became more realistic over time" (Bianchini \& Brenner 2009, p. 186).

Significantly, the participants were not taught a consistent definition of differentiated instruction by multiple presenters during their induction program, which included eight 3-hour seminars on the topic over two years (Bianchini \& Brenner, 2009), and what they were taught did not always fully align with Tomlinson's $(1999,2014)$ 
model. The researchers found that participants did not fully understand what differentiation was as a result of the seminars. They also noted that the new teachers did not move through a sequential and predictable continuum of development when it came to appropriating conceptions and practices related to differentiation (Bianchini \& Brenner, 2009). While one of the participants became increasingly aware of diversity issues and developed deeper understandings of and proficiency with instructional strategies that responded to diversity as she gained more experience during her first two years in the classroom, the other did not. Concluded the authors, "Our examination of differentiated instruction highlights how learning to teach is not necessarily synonymous with clear growth and refinement in understanding and skill over time" (Bianchini \& Brenner, 2009, p. 190).

\section{Learning to Differentiate across Contexts: Tomlinson et al. (1994, 1995, 1997)}

As described above, Tomlinson et al.'s (1995) larger mixed methods study examined the experiences of 85 novice teachers at six universities in multiple phases. None of the teacher preparation programs involved in the study required preservice teachers to take an entire course devoted to differentiation. In the first phases of the study, some preservice teachers received no treatment beyond the teacher preparation coursework presented by their universities $(n=41)$, while others were randomly assigned to treatment groups that either participated in a one-day workshop on differentiation $(n=22)$ or participated in a one-day workshop and received curriculum coaching during student teaching $(\mathrm{n}=23)$ (Tomlinson et al., 1994, 1995). In a subsequent phase of the study, researchers followed 10 participants from both control $(n=6)$ and treatment $(n=4)$ 
groups into the classroom during their first year of teaching (Tomlinson et al., 1995, 1997).

In this final phase of the study, researchers sought to identify the conditions that facilitated or inhibited new teachers' progress towards differentiation for academically diverse learners (Tomlinson et al., 1997). The researchers concluded:

Patterns evident throughout the study's data point to the enculturation of beginning teachers into a system largely inhospitable to the needs of academically diverse learners. Thus, even when the intervention novices held firm to beliefs in a need to modify instruction in ways responsive to academic diversity, the pull to do otherwise was almost overwhelming. (Tomlinson et al., 1997, p. 276)

New teachers entered a "standardized system with implicit rules and procedures" (Tomlinson et al., 1997, p. 277). Within such an "environment of uniformity, differentiation is an oxymoron" (Tomlinson et al., 1997, p. 277). Tomlinson et al. (1997) identified a number of factors presented by the standardized system that inhibited new teachers' progress towards differentiation, including the following.

- Curriculum was viewed as an exercise in content coverage, with students seen as passive receivers and repeaters of knowledge.

- Timing and pacing were standardized for all students.

- Assessments were standardized for all students, based on facts, and lacked formative approaches.

- Grading was a standardized comparison of learners.

- Advanced and struggling students were both viewed as problematic in light of standardized content to be covered and standardized pacing.

- Classroom management supported a standardized teacher-centered, rather than student-centered, classroom. 
Concluded the authors, "Novices succumb to the undertow of standardization in the face of an overwhelming demand for new skills" (Tomlinson et al., 1997, p. 277).

Tomlinson et al. (1995) echoed Bianchini and Brenner's (2009) conclusion that it cannot be assumed new teachers will grow in their understanding of academic diversity and improve their skills in responding to this diversity over time:

Given the complexities of teaching, the difficulties novices have making sense of classroom events, and the amount of experience needed to develop competence in the classroom, it might seem unrealistic to expect preservice teachers to provide differentiated instruction. On the other hand, patterns of teaching that form early in a career may become entrenched and thus never change...[I]t can be argued that introducing novices to student-centered views of instruction and giving them practice in implementing strategies may be necessary to break the one-size-fits-all conception of teaching. (Tomlinson et al., 1995, p. x)

While Tomlinson et al.'s $(1994,1995,1997)$ findings may begin to shed light on socialization factors that influence new teachers' conceptions and practice of differentiation, it must be noted that participants in the treatment group received a limited introduction to differentiation through a one-day workshop lasting only 6 hours and through curriculum coaching that the researchers characterized as not having a significant influence on participants. The researchers did not explore the depth of students' beliefs or personal background factors that may have influenced their implementation of the model, nor did the researchers consider the potential influence of teacher education experiences beyond those immediately related to the study.

\section{Theories Relevant to the Study}

In addition to the empirical literature described above, a framework comprised of two theories undergirds the present study. The situative perspective on teacher cognition (Putnam \& Borko, 2000) was the primary theoretical lens through which I collected and analyzed data and drew conclusions. Symbolic interactionism (Blumer, 1969) served as a 
foundational theoretical stance that informed my understanding and application of the situative perspective.

\section{Symbolic Interactionism}

Blumer (1969) describes the three premises of the theory of symbolic interactionism as: (a) "human beings act toward things on the basis of meanings that the things have for them" (p. 2), (b) "the meaning of such things is derived from, or arises out of, the social interaction that one has with one's fellows" (p. 2), and (c) "these meanings are handled in, and modified through, an interpretive process used by the person dealing with the things he encounters" (p. 2). This theoretical stance views reality as socially produced and emphasizes that actors socially construct meaning within a culture by interpreting people's actions (Woods, 1992). Symbolic interactionism reflects teaching as a complex social process in which meaning is made through interactions between teachers and others. The stance "assumes teachers and students together define the situation of the classroom, constrained by their backgrounds and the physical, temporal, and organizational context in which the classroom is embedded" (Brown \& Borko, 1992). Symbolic interactionism was used in the present study due to my focus on how novice teachers made meaning of differentiated instruction across contexts through interactions with multiple influential parties.

\section{Situative Perspective on Teacher Cognition}

Novice teachers learn to teach within multiple contexts, including coursework, preservice field experiences, and schools of employment. The situative perspective on teacher cognition (Putnam \& Borko, 2000) is a framework for the study of teacher learning that recognizes that the act of learning reflects both the individual learner and the 
social systems in which the learner is embedded (Borko et al., 2000). In this view, a novice's process of learning to teach is conceptualized as a trajectory through multiple social systems (Borko et al., 2000; Peressini, Borko, Romagnano, Knuth, \& Willis, 2004). Researchers using a situative perspective focus on how varied settings of teacher learning yield different types of knowing (Putnam \& Borko, 2000).

Situative perspective defined. The situative perspective (Greeno, 1997, 2003) asserts that both the learner and what is being learned are always situated within activities occurring in varied physical and social contexts. Thus, learning is located within particular social experiences and does not exist solely in the mind of the learner (Greeno, Collins, \& Resnick, 1996). In situative theories, learning is regarded as a social activity in which learners make sense of their experiences as they increase their authentic participation in the discourses and practices of a particular community (Greeno, 1997; Lave, 1988; Lave and Wenger, 1991). An examination of learning must therefore include a consideration of both explicit instruction in a practice and the learner's enculturation into a community's beliefs about that practice (Putnam \& Borko, 2000; Resnick, 1988). Because interactions between the learner and others in the environment are significant determinants of what is learned and how learning occurs (Putnam \& Borko, 2000), these interactive systems are the key focus of a study of the learner's enculturation.

The present study's research questions align with Greeno's (2003) call for future research grounded in the situative perspective: "The research agenda...[should] include more detailed studies that combine analyses of the informational and interpersonal aspects of students' participation in learning and that identify, in detail, how students'...knowledge and understanding grow through their sustained participation in 
learning activities" (p. 327). The situative perspective is a particularly useful lens through which to consider teacher development as new teachers are enculturated to adopt a school's prevailing norms and practices by observing and interacting with colleagues (Prescott \& Cavanagh, 2008).

Recontextualization. Research grounded in traditional cognitive perspectives (e.g., Schoenfeld, 1998) considers how and to what extent knowledge gained in one setting transfers to a different setting. This notion of transfer is incompatible with a situative perspective that sees learning as contextually embedded. Instead, research framed through a situative paradigm may "ask questions about the consistency of patterns of participation across situations, conditions under which successful participation in an activity in one situation facilitates successful participation in other types of situations, and the process of recontextualizing resources and discourses in new situations" (Peressini et al., 2004, p. 70).

Recontextualization involves transforming discourses and practices learned in one social context to embed them in a different social context (Adler \& Reed, 2002; Greeno, 1997). Learning involves improving attunement to the "constraints and affordance of activity systems" (Greeno, 1997, p. 12). If, when the learner initially learned the practice, the learner was attuned to the constraints and affordances that are the same in the second context, recontextualization will occur more easily.

Longitudinal research on novice teachers conducted from a situative perspective seeks to examine how practices learned in teacher preparation programs are recontextualized in schools of employment (Peressini et al., 2004). New teachers appear to recontextualize diverse practices learned in multiple contexts through attunement to 
the constraints and affordances of those contexts (Brouwer \& Korthagen, 2005; Peressini et al., 2004). Nolen et al. (2011) argue: "By reframing transfer as recontextualization, we consider the particular norms, values, patterns of interactions, identities, and structures that afford and constrain how knowledge is created and how practices are realized" (p. 89).

\section{Research Questions}

The literature reviewed in this chapter grounds the present study's research questions. No identified studies to date have examined how preservice teachers make meaning of Tomlinson's $(1999,2014)$ model of differentiated instruction in a teacher preparation course in which differentiation serves as the primary focus of instruction, allowing students to consider the model in depth. Likewise, no identified studies to date have examined how novice teachers make meaning of Tomlinson's model of differentiation across both a teacher preparation course in which differentiation serves as the primary focus of instruction and experiences in their early teaching careers. The present study addressed these gaps in the literature through two general qualitative questions reflecting the study's two phases and five more focused questions:

\section{Phase 1}

1. How do preservice teachers enrolled in a course on differentiated instruction in a teacher preparation setting make meaning of Tomlinson's $(1999,2014)$ model of differentiation?

(a) What are their conceptions of the model when they first enter the course, if any, and how do their conceptions change during the course, if at all? 
(b) How do factors external to the course relate to their conceptions of the model?

\section{Phase 2}

2. How do teachers who completed a course on differentiated instruction in a teacher preparation setting make meaning of Tomlinson's $(1999,2014)$ model of differentiation during the fall of their first year in the classroom?

(a) What are their conceptions of the model as they first enter the classroom, and how do their conceptions change during the fall, if at all?

(b) What is the relationship between their conceptions of the model and their teaching practice?

(c) How do contextual factors relate to their conceptions of the model and their teaching practice? 


\section{CHAPTER THREE}

\section{METHOD}

This chapter provides a description of my research design rationale and participant selection process, an overview of data collection and analysis methods, an outline of the criteria for evaluating the study, and a researcher as instrument statement.

\section{Research Design Rationale}

A qualitative research design is appropriate when an issue warrants exploration to develop a complex, detailed understanding, and when the voices of individuals involved with the issue will illuminate that understanding (Creswell, 2013). The focus of the present study, how preservice teachers and first year teachers made meaning of differentiation, therefore warranted such a research design. The study's examination of novice teachers across multiple learning-to-teach contexts responded to the field's calls for more longitudinal designs (Ball, 1988; Borko \& Putnam, 1996; Bryan and Atwater, 2004; Nolen et al., 2011; Thompson et al., 2013; Wideen et al., 1998). This exploratory multicase study (Stake, 2006) was conducted in two phases.

The research site for Phase 1 was a graduate-level course on differentiated instruction offered through the Curry School of Education at the University of Virginia, a mid-sized public research university. Data collection for Phase 1 occurred between January 9 and May 9, 2014. This phase explored how preservice teachers enrolled in a course on differentiated instruction in a teacher preparation setting made meaning of the 
model. Students' conceptions of the model were examined at multiple points throughout the semester so that changes in their conceptions could be considered. How contextual factors outside of the course may have been related to their conceptions was also examined.

Data collection for Phase 2 occurred between August 25 and December 11, 2014. This phase explored how two of the participants studied in Phase 1 continued to make meaning of the model during the fall of their first year in the classroom. The use of an exploratory multicase study research design was warranted by the deep examination of how participants' conceptions of the model developed over time, what the relationship was between their conceptions of the model and their practice, and how different contextual factors were related to their conceptions and practice.

\section{Case Study Rationale}

A case study is an in-depth empirical inquiry into a complex contemporary social phenomenon in its real-world context (Yin, 2009). It presents an intensive and holistic description and analysis of a phenomenon (Merriam, 1988). Through this intensive focus on a single phenomenon, the interactions of significant factors that characterize that phenomenon are uncovered (Merriam, 1998). The case is the object of study chosen to illuminate the phenomenon. It presents a "bounded system" (Smith, 1978), or a delimited entity within a bounded context. Thus, a case researcher often attempts to identify the boundaries that separate the case from its context (Stake, 2006).

A case study research design is appropriate when a researcher intends to study a contemporary phenomenon over which she has no control (Yin, 2009). It is particularly fitting when unique contextual conditions are critical to the study of the phenomenon 
(Yin, 2009; Yin \& Davis, 2007), or when the variables influencing the phenomenon cannot be easily separated from the context (Merriam, 1998). The result is a "rich and holistic account of a phenomenon" (Merriam, 1998). Exploratory case studies are used to develop propositions for additional research and are appropriate when the existing knowledge base surrounding a phenomenon is weak (Yin, 2009), as was the case, to some extent, for the present study.

Multicase studies examine several connected cases in depth to illuminate a common phenomenon, with a focus on issues that cut across the cases and best lead to an understanding of the phenomenon (Stake, 2006). Multicase projects are an appropriate research design when the aim is to understand the phenomenon in varied contexts. Both situational uniqueness of individual cases and common characteristics across cases are therefore considered (Stake, 2006). Stake (2006) distinguishes multicase studies from comparative case studies, noting, "Comparison is a competitor to a probing study of a case. It...usually fixes attention on one or two variables. In doing so, it obscures the situationality and complex interaction of case knowledge" (p. 83). Thus, the study's aim of developing an in-depth understanding of how two novice teachers made meaning of differentiation across contexts merited an exploratory multicase study design. Each of the participants served as one case; one teacher was the unit of analysis.

\section{Research Paradigm}

My study of participants' meaning-making processes was informed by my constructivist or naturalistic paradigm (Lincoln \& Guba, 1985). This paradigm assumes multiple social realities that are apprehendable through multiple personal and social constructions (Schwandt, 2000). Knowledge is viewed as being co-constructed through 
the interaction of the researcher and the participant. These ontological tenants lead to the epistemological question: What is the meaning of the phenomenon of interest for the people engaged in that phenomenon? Multiple perspectives of the phenomenon must be considered due to the ontological belief that multiple social realities exist.

Interpretive qualitative inquiry emphasizes individuals" "sense-making" (Erickson, 1986, p. 127) considered within a wider social context. It is an appropriate approach to research when the goal is to learn more about the meaning perspectives of individuals involved in particular events (Erickson, 1986), as it is primarily focused on understanding the complex meanings of a particular phenomenon that different people construct through interaction with others or with various cultural norms (Merriam, 1998; Creswell, 2013). Interpretive studies treat phenomena holistically (Schwandt, 1994). Data are used to create new conceptual categories or to illustrate, reinforce, or challenge theoretical assumptions (Merriam, 1998). Interpretive studies are characterized by their theoretical orientations, as well as the centrality of deep and complex interpretation (Erickson, 1986). To gain access to participants' subjective knowledge, methods must include substantial interaction with them. Since the focus of my study was participant meaning-making, my data collection methods emphasized dialogue with the participants.

\section{Context and Researcher Role}

Both of the participants graduated from their degree programs in May 2014 with a Master of Teaching (MT) degree. Karen ${ }^{3}$ graduated from the two-year postgraduate master of teaching program (PG/MT), while Nicole graduated from the five-year program for undergraduates (B/MT). Preservice teachers in the PG/MT program received undergraduate degrees in fields other than education before pursuing their MT. When

\footnotetext{
${ }^{3}$ All participants, schools, and districts have been given pseudonyms.
} 
they graduated in May 2014, preservice teachers in the B/MT program had both an MT degree and a Baccalaureate degree in a field other than education conferred upon them.

Degree program requirements. Preservice teachers in the $\mathrm{B} / \mathrm{MT}$ program (BMTs) take education courses in their third, fourth, and fifth years of study and complete field placement requirements throughout that period. In their third and fourth years, BMTs spend several hours per week in their field placements, with a different placement each semester for four semesters. In the third year, they generally observe in their placements or provide individual students with one-on-one support. In the fourth year, they usually teach one short lesson to their placement classes in the fall and around five short lessons to a different placement class in the spring. BMTs student teach in the fall of their fifth year. Nicole had a split placement for her student teaching due to participation in a program to student teach abroad. Thus, Nicole graduated having had six field placements over three years, with the degree of teaching responsibility increasing with each placement.

Preservice teachers in the PG/MT program (PGMTs) take only education courses while pursuing their degrees and complete field placement requirements throughout that two-year period. In their first year, PGMTs spend several hours per week in their field placements, with two different placements each semester, for a total of four placements that first year. Each semester in the first year, PGMTs observe or provide individual students with one-on-one support in one placement, while the other placement involves teaching a handful of short lessons. PGMTs student teach in the fall of their second year. Karen did not have a split placement for her student teaching. Thus, Karen graduated 
having had five field placements over two years, with the degree of teaching responsibility increasing between first and second year placements.

Differentiating Instruction course. During their final semester of coursework after completing student teaching, University of Virginia students in the B/MT and PG/MT programs typically take a lighter course load, with limited course requirements compared to previous semesters; however, Nicole took the Differentiating Instruction course as an elective, going beyond her program requirements. Karen was required to take the course for her add-on endorsement in gifted education.

The class met once a week for two and a half hours for a total of 14 class meetings (see Appendix A). This graduate-level course presented an overview of the key principles forming the conceptual framework of differentiated instruction (Tomlinson, 2014; Tomlinson \& Imbeau, 2010), as well as key teaching practices informed by those principles. Course content was presented through lecture, whole group discussion, small group discussion, and numerous workshop sessions on specific instructional strategies. Many of the workshops were differentiated based on student readiness, interest, or preferences for working configurations, such working with a small group, with a partner, or individually. Video clips of K-12 teachers engaged in classroom instruction were also shown to illustrate core principles and practices of differentiation. Major assessments included writing a synthesis of the key principles of differentiation after reading several texts on the model; developing three differentiated lesson plans that modified instruction based on readiness, interest, or learning profile; and creating a final product of the students' choice that demonstrated their understanding of the model's key principles and related practices. Students also wrote five reflections throughout the semester in which 
they described their developing conceptions of differentiation and made connections to topics and contexts beyond the course in response to prompts (see Appendix B).

Throughout the course, great attention was paid to helping students "distinguish between 'strategies' and organizational structures associated... with differentiated instruction and the underlying educational principles that constitute them" (Sherman, 2009, p. 50), reflecting a belief that "techniques alone cannot sustain progressive educational environments" (Sherman, 2009, p. 50). Key principles and practices of differentiation were intentionally modeled for students throughout the course (Santangelo \& Tomlinson, 2012).

It should be noted that Dweck's (e.g., 2000, 2007) work on mindset was a key topic addressed by the course. A large portion of the third class meeting was devoted to a discussion of research on mindset and its implications for the learning environment in a differentiated classroom (see Appendix A). ${ }^{4}$

The course was taught by Tomlinson while I assisted with the class, attending all class meetings and serving as the lead instructor for one of those classes. I did not assign grades to assessments, but I did provide students with individualized feedback on their lesson plans and their ongoing planning for their final projects.

\section{Participant Selection}

This section describes how participants were selected for each of the study's phases.

\footnotetext{
${ }^{4}$ This was not participants' first exposure to the concept of mindset. Karen had read Dweck's (2007) book Mindset before the Differentiating Instruction course (course entrance survey, 1/13/14). At the beginning of the course, Nicole had not read any of Dweck's work and did not recall discussing it in any other coursework (personal communication, 4/14/15). However, all students in the school of education had been made aware of Dweck's work in 2012-2013 when they were invited to participate in discussion groups of Mindset and when Dweck visited the campus as an invited lecturer. Nicole began reading Mindset during spring 2014 after the third meeting of the Differentiating Instruction course and completed the book during summer 2014 (personal communication, 4/14/15).
} 


\section{Phase 1 Selection}

Of the 24 enrolled students in the Differentiating Instruction course, 20 consented to participate in the Phase 1 study. Because time limitations prevented my interviewing all 20 at multiple time points, roughly half the sample was selected for inclusion in a focal sample for Phase 1 based on the possibility of longitudinal follow-up in Phase 2. This selection was based on three criteria. The first criterion, which four participants did not meet, was that as of January 2014, they planned to teach in fall 2014. The second criterion was anticipated geographic proximity to the university in the fall. Four additional participants did not meet this criterion, as they planned to teach in other regions of the country or abroad. The third selection criterion, which one student did not meet based on apparent uneasiness with the interview process, was that participants seemed likely to serve as information-rich cases. The application of these selection criteria following first-round screening interviews with all 20 participants yielded 11 focal participants for Phase 1, including Karen and Nicole.

\section{Phase 2 Selection}

In Phase 2, purposeful sampling was used to select cases who were most likely to illuminate the research questions (Yin, 2009).

Selection criteria. First, only focal participants who secured teaching positions in the mid-Atlantic region were considered due to the necessity of geographic proximity. The second selection criterion was whether participants assumed positions in general education settings. Because the present study sought to identify how new teachers made meaning of differentiated instruction in a general education setting reflecting a broad 
range of academic diversity, the five focal participants who were hired to teach special education were not considered for Phase 2.

The application of these two selection criteria yielded six potential cases for longitudinal follow-up. In summer 2014, I invited those six individuals to participate in Phase 2. Five consented. These included two public high school social studies teachers and three elementary teachers - one in a public school (Nicole) and two (Karen and another teacher) in the same private school. During the month before school began, I arranged a brief interview with each of the five participants to screen candidate cases (Yin, 2009) based on whether all the participants still appeared likely to serve as information-rich cases, and all did. In the fall, I gained school access for every participant and conducted a screening observation in their classrooms to ensure that they were not so overwhelmed by their responsibilities that they would be unable to serve as information-rich cases. Again, no cases were removed from consideration as a result of this screening.

Ultimately, I selected Nicole, a third grade teacher in a public school, and Karen, a kindergarten teacher in a private school, as cases for the present study because I believed they would best enhance my understanding of the phenomenon of study, reflect the different contexts in which the phenomenon occurred, and provide the richest opportunities for me to learn about those contexts (Stake, 2006). It appeared that selecting either the secondary social studies participants or the elementary participants as cases for in-depth study would prove the most illuminating for cross-case analysis. Because I did not gain access to one of the secondary teachers' classrooms until October, halfway through the fall, my observation data for that participant was limited in volume 
and scope compared to that of the other participants. For this reason, I narrowed my focus to the elementary participants. It seemed that the contrast of public and private settings might provide the best opportunities to study participant meaning-making in varied contexts, so I then concluded that Nicole and one of the two teachers from the private school would serve as my cases. Last, I selected Karen as the second participant most likely to meaningfully illuminate the research questions in light of cross-case analysis. This was because, in comparison to Nicole and Karen, the instructional practice of the other participant at Karen's school may have been structured significantly differently due to potential co-teaching with the other teacher at her grade level or with the experienced certified teacher who served as her teaching assistant.

\section{Data Collection}

Data for the study were collected through interviews, observations, and documentation. This section describes data collection procedures for each phase separately. All data were stored in compliance with the University of Virginia's data protection policies. Table 3 identifies the data sources used to answer the focus research questions for each phase. 
Table 3

Focus Research Questions with Corresponding Data Sources

Focus Research Questions

Data Sources

Phase 1

1a. What are participants' conceptions of Tomlinson's $(1999,2014)$ model of differentiation when they first enter the course, if any, and how do their conceptions change during the course, if at all?

1b. How do factors external to the course relate to their conceptions of the model?

Phase 2

2a. What are participants' beliefs about the model as they first enter the classroom, and how do their beliefs change during the fall, if at all?

$2 \mathrm{~b}$. What is the relationship between their beliefs about The model and their teaching practice?

2c. How do contextual factors relate to their beliefs about the model and their teaching practice?
- Participant interviews

- Course assignments

- Informal observations
- Participant interviews

- Course assignments

- Informal observations
- Participant interviews

- Classroom artifacts

- Participant interviews

- Classroom observations

- Classroom artifacts

- Participant interviews

- Interview with administrator or mentor

- Classroom observations

- Classroom artifacts

- Publicly available school, district, and state documents

\section{Phase 1 Data Collection}

Data collection for Phase 1 occurred between January 9 and May 9, 2014. Oneon-one semi-structured interviews with participants, informal participant-observation in class, and students' course assignments were used to gather data. 
Interviews. I interviewed each of the 11 focal participants, including Karen and Nicole, four times during the course, spread evenly throughout the semester. The other nine participants were interviewed twice, participating in the first and fourth rounds. Interviews ranged in length from around 25 to 50 minutes, with the average interview lasting around 40 minutes. Interviews with Karen ranged from 25 to 35 minutes, totaling 125 minutes. Interviews with Nicole ranged from 25 to 45 minutes, totaling 155 minutes. Questions were designed to elicit information about participants' meaning-making processes surrounding their developing conceptions of differentiation.

The first round of interviews occurred in late January. In this interview, participants were asked to explain their general philosophy of teaching and learning, ideal teaching setting, and initial conceptions of differentiation. The second round occurred in late February. Questions in this interview focused on participants' conceptions of the principles of differentiation involving learning environment and quality curriculum, the two major topics addressed by that point in the course, and on their field placement experiences. The third occurred in early April. Interview questions in this round asked participants to explain their conceptions of the principles of differentiation involving ongoing assessment; the modification of instruction based on readiness, interest, and learning profile; and leading and managing a differentiated classroom, which were the three major topics addressed in the course since round two interviews. Round three questions also addressed participants' own K-12 educational experiences and personal backgrounds. The fourth round occurred in early May, after participants' final products for the course had been submitted. Fourth round interview questions asked participants to reflect on how their conceptions of differentiation evolved throughout the course and 
re-posed some of the questions asked in the first interview to examine potential changes in conceptions. As part of this final interview, I also asked participants to draw and explain a concept map illustrating the relationship among the five non-negotiable elements of a differentiated classroom: a supportive learning environment; high quality curriculum; ongoing assessment; modification of instruction by readiness, interest, or learning profile; and effective leadership of students and management of classroom details.

All interviews were conducted using semi-structured protocols (see Appendices C-F). Interviews were audio recorded, and I wrote analytic notes about the interviews within 24 hours of conducting them. I later transcribed the interviews using word processing software.

Observations. I also conducted informal participant-observation (Yin, 2009) while attending class meetings. I observed the participants as they listened and asked questions during lectures and as they worked with partners or small groups in discussions or workshops. During discussions and workshops, I often interacted with participants to answer questions or participate in discussions. While my responsibilities as the course assistant did not allow me to record formal, detailed field notes, I used word processing software to record verbatim the questions focal participants raised during whole-class lectures and to paraphrase some comments that focal participants made during small group discussions or workshops.

Documentation. Written assignments that students completed for the course served as the third source of data for Phase 1. These included the major course assessments of a synthesis of the key principles of differentiation, three lesson plans 
reflecting modified instruction, and the final product, as well as reflections in which students described their developing conceptions of key ideas and made connections to topics and contexts beyond the course (see Appendix B). Students submitted all assignments electronically through the university's online course platform, to which I had access as the course assistant. After the course concluded, I downloaded copies of the participants' assignments for analysis.

\section{Phase 2 Data Collection}

Data collection for Phase 2 occurred between August 25 and December 11, 2014. One-on-one, semi-structured interviews with participants were used to gather data throughout this period. After gaining access to participants' schools, interviews with Karen's principal and Nicole's mentor, direct observations in classrooms, classroom artifacts, and publically available documents about participants' schools, districts, and curricular standards were also used.

Interviews. I conducted five one-on-one interviews with participants during the fall, with an interview occurring around once every four weeks. Interviews with Karen ranged from 25 to 40 minutes, totaling 165 minutes. Interviews with Nicole ranged from 20 to 45 minutes, totaling 145 minutes. Interviewing participants approximately once a month allowed me to regularly examine changes in participants' conceptions and practice over time, while ensuring that the costs borne by the participants were not excessive in light of ethical considerations (Miles \& Huberman, 1994).

As discussed above, I used an initial informal interview, conducted through video conferencing software during the month before school began, to screen candidate cases (Yin, 2009) based on whether the participants still appeared likely to serve as 
information-rich cases for Phase 2. Questions during this shorter interview, around 15 to 20 minutes, addressed how summer experiences such as trainings may have influenced their thinking about differentiation, the nature of the academic diversity they anticipated encountering in their classes, general information about the school, and support for new teachers.

The remaining four interviews, conducted using a semi-structured protocol, were held face-to-face in the participants' classrooms during or after the school day immediately following observations. Questions during the first, second, and third of these interviews (see Appendix G) addressed the lesson I had just observed, the degree to which participants viewed their conceptions of differentiation as evolving, the relationship between those conceptions and their current practice, and how individual contextual factors influenced their conceptions and their practice. The semi-structured protocol included as Appendix G contains questions that I asked during the first, second, or third interviews depending on the nature of the lesson I had just observed, information the participant volunteered about her experiences since our last interview, and how much time we had to complete the interview.

During the second interview, participants were asked to engage in the same concept map activity that they completed during the fourth interview of Phase 1 (see Appendix F). After creating a new concept map reflecting their conceptions of differentiation as of October 2014, they were shown their concepts map from May 2014 and asked to identify and explain any similarities or differences between the two products (see Appendix G). 
The fourth interview targeted how contextual factors influenced participants' conceptions of differentiation and their related practice. In preparation for this interview, I conducted an initial analysis of all data gathered from both phases of the study for each participant and identified every factor that participants had suggested might have a relationship to their conceptions of the model or their attempts, or lack of attempts, to implement it. During the interview, I provided participants with a list of these factors, along with the dates on which they had made the comments (see Appendices H-I). I asked them to identify whether or not the factor had significantly influenced their conceptions or implementation of differentiation in positive or negative ways and to explain their reasoning for each factor.

I also conducted interviews with Karen's principal and Nicole's school-appointed mentor. Questions were designed to elicit information relating to contextual factors that may have influenced participants' beliefs about the model and their teaching practice. I selected Karen's principal and Nicole's mentor as the best school member to interview for the study based on the participants' recommendations after I explained the purpose of the interview in mid-fall. I asked participants which administrator, teacher leader, or peer provided them the most support as a new teacher and would be their first resource for questions about curriculum and instruction, including differentiation. Additional data from participant interviews and from interviews with these colleagues confirmed that Karen's principal and Nicole's mentor were the best sources for information about contextual factors. I used a semi-structured protocol (see Appendix J) for these interviews, which occurred at their schools during the school day and which lasted 30 minutes for Nicole's mentor and one hour for Karen's principal. 
All interviews were audio recorded, and I wrote analytic notes about the interviews within 24 hours of conducting them. I then transcribed the interviews using word processing software.

Observations. Kaplan (1991) noted the critical importance of direct observation of teaching performance when researching teacher practice or teacher belief. After gaining access to participants' schools, I also conducted four classroom observations, with one occurring around once every four weeks. I conducted interviews with participants immediately after my observations so that I could pose follow-up questions about observed events. When possible, each of my observations included at least one full lesson. Observations of Karen's classroom ranged from 45 to 100 minutes and totaled four hours and 40 minutes. Observations of Nicole's classroom ranged from 40 to 95 minutes and totaled just over four hours. During these visits, I largely assumed the role of a passive direct observer, not participating in the events being observed (Yin, 2009). Observation times were mutually agreed upon. Around one week in advance, I suggested a date and time for the next observation and interview round, which the participants generally agreed to. I scheduled visits at varied times throughout the school day and observed participants teach multiple subjects. For both participants, dates for Phase 1 and Phase 2 interviews and for Phase 2 classroom observations, along with the subjects taught during the Phase 2 observations, are listed in Tables 4 and 5.

I recorded field notes during all observations by hand. Field notes included records of verbal statements made by those present, as well as descriptions of the setting, general activity, and non-verbal interactions among those present. Within 24 hours of each observation, I expanded and analyzed my field notes in write-ups composed with a 
word-processing program.

Documents. Two classroom artifacts were collected during the fall of Phase 2, a photograph of a chart in Karen's classroom used to inform her students about daily learning station rotations for language arts and a copy of Karen's math pacing guide for the first quarter. I also consulted publically available school, district, and state documents when considering contextual factors that may have influenced participants' beliefs about the model or related practice. These included websites for Nicole's school and district, standards for third grade subjects in public schools in Nicole's state, and the kindergarten and first grade Common Core Standards for English Language Arts and Mathematics used in Karen's school. 
Table 4

Karen Interview Dates and Classroom Observation Dates with Lesson Subjects

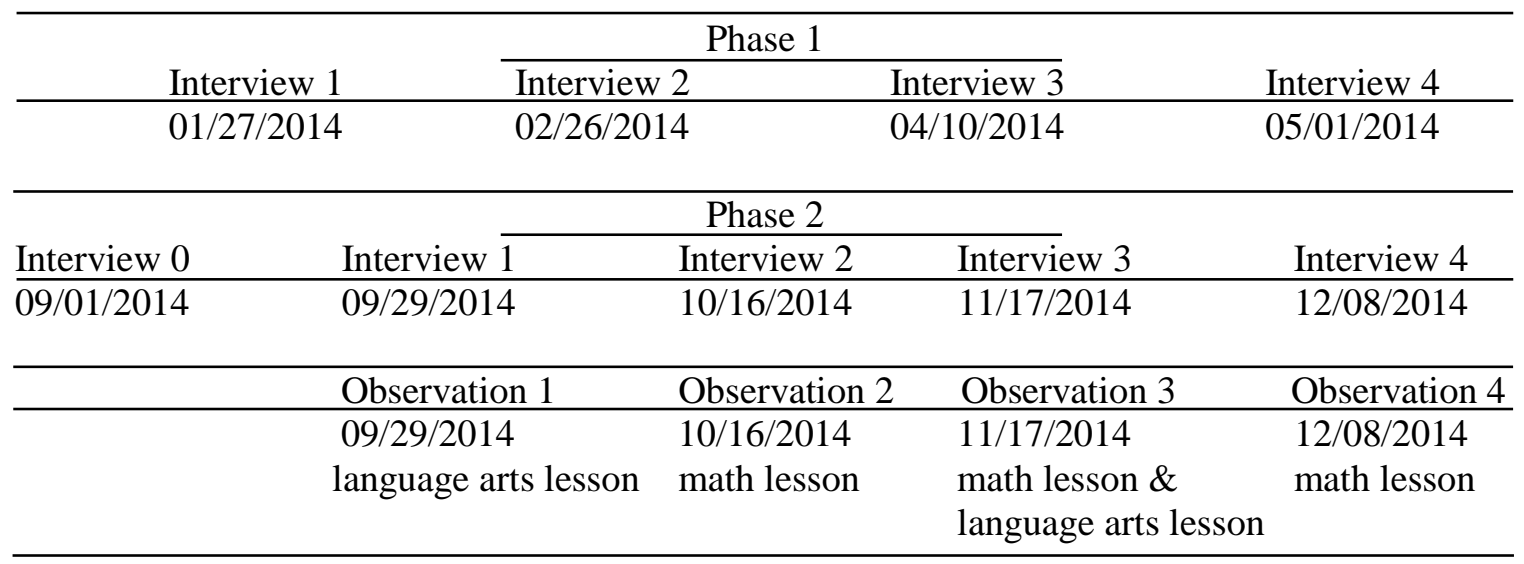

Note. Phase 2 Interview 0 was an informal 20-minute interview to screen potential Phase 2 participants.

Table 5

Nicole Interview Dates and Classroom Observation Dates with Lesson Subjects

\begin{tabular}{|c|c|c|c|c|}
\hline \multirow{2}{*}{\multicolumn{2}{|c|}{ Interview 1}} & \multicolumn{2}{|l|}{ Phase 1} & \multirow[b]{2}{*}{ Interview 4} \\
\hline & & Interview 2 & Interview 3 & \\
\hline \multicolumn{2}{|c|}{$01 / 27 / 2014$} & $02 / 24 / 2014$ & $04 / 10 / 2014$ & $05 / 01 / 2014$ \\
\hline \multirow{3}{*}{$\frac{\text { Interview } 0}{08 / 25 / 2014}$} & & \multicolumn{2}{|l|}{ Phase 2} & \multirow[b]{2}{*}{ Interview 4} \\
\hline & Interview 1 & Interview 2 & Interview 3 & \\
\hline & $09 / 30 / 2014$ & $10 / 20 / 2014$ & $11 / 14 / 2014$ & $12 / 09 / 2014$ \\
\hline \multicolumn{2}{|c|}{ Observation 1} & Observation 2 & Observation 3 & Observation 4 \\
\hline \multirow{2}{*}{\multicolumn{2}{|c|}{$\begin{array}{l}\text { 09/30/2014 } \\
\text { language arts lesson }\end{array}$}} & $10 / 20 / 2014$ & $11 / 14 / 2014$ & $12 / 09 / 2014$ \\
\hline & & $\begin{array}{l}\text { science lesson \& } \\
\text { social studies lesson }\end{array}$ & language arts lesson & language arts lesson \\
\hline
\end{tabular}

Note. Phase 2 Interview 0 was an informal 25-minute interview to screen potential Phase 2 participants. 


\section{Data Analysis}

Data analysis is an iterative process (Lincoln \& Guba, 1985). For both phases of the study, the multicase study analytic approach (Stake, 2006) was supported by techniques of constant comparison (Charmaz, 2006; Glaser \& Strauss, 1967).

\section{Analytic Memoing}

Throughout the process of data gathering, I composed analytic memos to ensure analysis occurred from the earliest stages of collection. Through this ongoing analytic memo writing, sequential comparisons of data at each level of analytic work were accomplished (Charmaz, 2006). Charmaz (2006) noted, "Memo-writing forms a space and place for exploration and discovery" (p. 93) of the data's meaning. This occurs as deep connections are made to the literature and themes emerge. I used memos to compel myself to make conceptual leaps from the raw data to abstract analysis, ultimately moving away from an examination of specific incidents to look more broadly at a case, and then away from an examination of a specific case to look more broadly at themes across cases.

\section{Unitizing and Categorizing}

I used start codes grounded in the literature to guide decisions surrounding data unitizing through deductive provisional coding (Miles \& Huberman, 1994; Miles, Huberman, \& Saldana, 2014). Units of data from interviews, observations, and documents were compared with other related data units (Lincoln \& Guba, 1985). To support this unitizing process, I coded data from both phases using NVivo qualitative analysis software. Through inductive coding, I refined my list of codes, clarifying the meanings of some and adding others (Miles, Huberman, \& Saldana, 2014). Based on 
these unit comparisons, I developed tentative categories that captured a recurring pattern across the data (Miles, Huberman, \& Saldana, 2014). These categories were repeatedly tested and refined as additional data were gathered and analyzed (Charmaz, 2006).

Conducting a multicase study necessitated both within- and cross-case analysis. When engaged in within-case analysis, I considered each case as comprehensive unto itself and sought to learn as much as possible about the contextual variables of that individual case (Merriam, 1998). After within-case analysis was completed, I then engaged in cross-case analysis to build abstractions across cases (Merriam, 1998). I avoided superficial summarization of a few themes but instead "look[ed] carefully at the complex configuration of processes within each case [and] the local dynamics...to see patterning of variables that transcended particular cases" (Miles \& Huberman, 1994, pp. 205-206). Ultimately, emerging categories led to the development of cross-case themes and assertions through the multicase study analytic approach (Stake, 2006). I gave special focus to the analytic techniques of examining of rival explanations and disconfirming evidence to strengthen findings and using time-series analysis to trace changes in participants' understandings over time (Yin, 2009).

In the next two chapters, I present findings reflecting within-case analyses for Karen and Nicole across Phases 1 and 2 of the study, while Chapter Six includes crosscase themes, cross-case assertions, and implications.

\section{Criteria for Evaluation of Findings}

Lincoln and Guba's (1985) trustworthiness criteria were used to guide the development, presentation, and interpretation of the findings. 


\section{Credibility}

The first criterion of credibility involves demonstrating that the researcher has adequately represented participants' multiple constructed realities (Lincoln \& Guba, 1985). In this study, credibility was enhanced by triangulation of data sources, member checking, and prolonged engagement (Merriam, 1998), as well as the consideration of rival explanations (Yin, 2009).

Data source triangulation. The goal of triangulation is to confirm that the right interpretations have been made of the right data (Stake, 2006). With data source triangulation, multiple sources of evidence are compared to see whether the phenomenon or case retains the same meaning when it occurs in or experiences different circumstances (Stake, 1995). The goal is to corroborate a fact or phenomenon by collecting and comparing relevant information from more than one source and achieve "converging lines of inquiry" (Yin, 2009, p. 115). The data collection strategy for the present study was informed by the goal of triangulation. While participant interviews and course assignments served as the primary data sources to address the research questions posed in Phase 1, data from informal class observations were included to support potential converging lines of inquiry. In Phase 2, interviews with Karen's principal and Nicole's mentor were included for triangulation purposes to support research question $2 \mathrm{c}$ regarding contextual factors that may relate to teachers' beliefs about the model or related practices. Likewise, classroom artifacts were considered as potential data sources for Phase 2 to support triangulation for research question $2 b$, which involves the relationship between beliefs and practice. As I engaged in data analysis, I repeatedly examined converging lines of inquiry across multiple sources. 
Member checking. Member checking invites participants to review transcripts, notes, or drafts of writing for accuracy of facts and plausibility of tentative interpretations (Lincoln \& Guba, 1985; Merriam, 1998; Stake, 1995). This process may improve data interpretation or yield new data for the study (Stake, 2006).

On at least two occasions, I asked each participant to review short sections of transcripts or field note write-ups for which I believed the accuracy of information could be improved. As discussed above, in the final interview of Phase 2, I also asked the participants to review tentative interpretations from within-case analyses addressing question $2 \mathrm{c}$ about how potential contextual factors may have influenced their conceptions of the model and their teaching practice. I offered participants the opportunity to tell me whether the factors I had identified in my analysis in their opinion had no significant influence, had a supportive influence, or had a challenging influence, or whether they were unsure about the influence it may have had. During this member checking exchange, both Karen and Nicole clarified my interpretation of several factors, saying they had little or no influence when previous data might have suggested otherwise.

Prolonged engagement and rival explanations. Additionally, prolonged engagement through repeated contact with participants during observations and interviews in both phases at multiple time points supported the study's credibility, as it provided multiple opportunities to examine the same phenomenon (Merriam, 1998). Finally, as noted above, addressing rival explanations was emphasized in data analysis.

\section{Transferability}

The second criterion of transferability involves providing adequate descriptive data about the context in which an inquiry was conducted such that readers may make 
judgments about the possibility of transfer (Lincoln \& Guba, 1985). Readers must be able to determine how closely their situations and the research situation match and thus whether findings can be transferred (Merriam, 1998). Lincoln and Guba (1985) propose that the degree of transferability is a function of "fittingness" (p. 124), or the similarity between the contexts of the research situation and the reader's situation. To enhance transferability in the present study, I reported findings, including descriptions of contextual factors influencing participants in both phases, using "thick description" (Geertz, 1973) to provide sufficient detail to assist readers in assessing fittingness.

\section{Dependability}

Dependable findings are consistent with collected data (Lincoln \& Guba, 1985). Distinguishing dependability from reliability, Merriam (1998) explains: "[R]ather than demanding that outsiders get the same results, a researcher wishes outsiders to concur that, given the data collected, the results make sense - they are consistent and dependable" (p. 206). To address dependability, an audit trail was developed. I have included in this paper detailed descriptions of data collection and key interpretive decisions (Merriam, 1998). Throughout the study, I also developed a formal database and maintained a chain of evidence that would allow an external observer to trace the derivation of evidence from research questions to final study conclusions (Yin, 2009).

\section{Confirmability}

The fourth criterion is confirmability, which refers to whether or not the data themselves are confirmable or confirmed (Lincoln \& Guba, 1985). Maintaining an audit trail and data source triangulation, discussed above, both support confirmability, as does keeping a reflexive journal (Lincoln \& Guba, 1985). I maintained a reflexive journal 
during both phases of the study to reflect on key methodological decisions and on issues that arose from my serving as the research instrument.

\section{Researcher as Instrument}

Qualitative research is inherently subjective, as the instrument through which data are perceived, gathered, and analyzed is the researcher (Lincoln \& Guba, 1985; Stake 1995). While Guba and Lincoln (1981) have argued that humans are uniquely qualified to serve as the instrument for constructivist research, the challenges posed by the researcher's assumption of this role must also be addressed. Because, as an interpretivist, I recognized it is impossible to refrain from bringing my own subjectivities into the field, I sought to maintain an awareness of how these subjectivities may have influenced my research.

Stake (2006) noted that it is common for researchers who conduct qualitative case studies to care about or believe in the case or the phenomenon of study. Case researchers often hope to find that a program works and are "disposed to see evidence of success" (Stake, 2006, p. 86). I pursued doctoral studies to study Tomlinson's (1999, 2014) model in depth and am Tomlinson's academic advisee, with a degree specialization in differentiated instruction. I have worked extensively with the model throughout my graduate studies. As a former middle school teacher, I differentiated instruction in my own classroom in response to student needs and therefore recognized its value on a personal level. As the assistant for the Differentiating Instruction course, I assumed a coaching role with the participants and grew to care about their success as educators. I also believe in the potential of differentiated instruction to significantly improve the 
learning experiences of America's K-12 students by respecting and accommodating student differences.

As a result, throughout the course of the study, I remained mindful of my own background, interests, and potential biases, and of the effects those may have had on my perceptions and interpretation of the data. Through a constant focus on reflexivity, I worked to ensure that my own beliefs about differentiation and my hopes for the participants as they began their careers did not cloud what I perceived or recorded. I maintained an awareness that, as Stake (2006) noted, qualitative studies are value-laden, and researchers often tend to underplay negative findings. This helped me avoid focusing only on data that might have led to the conclusions I anticipated or preferred. My focus remained "the specifics of action and of meaning-making perspectives" (Erickson, 1986, p. 124) of the participants, rather than meanings I might have ascribed to a phenomenon. 


\section{CHAPTER FOUR}

\section{FINDINGS FOR FIRST PARTICIPANT}

This dissertation includes findings from longitudinal case studies of two participants, Karen and Nicole, across Phases 1 and 2 of the larger study. This chapter presents findings about Karen's experiences in each phase. Findings are presented in response to each of the five focal research questions.

\section{Karen's Phase 1 Experiences}

At the beginning of the Differentiating Instruction course in January 2014, Karen was a 24 year old full-time student in the two-year postgraduate master of teaching (PG/MT) program. Karen had graduated with a bachelor's degree in another field from a different higher education institution in spring 2012 and enrolled in the University of Virginia’s PG/MT program in fall 2012. During Phase 1 of the study in spring 2014, Karen was in her last semester of coursework, having just completed a semester of fulltime student teaching in a second grade classroom in fall 2013. She sought an elementary education (K-6) endorsement as well as an add-on gifted education (K-12) endorsement. Karen is a Caucasian female.

\section{Research Question 1(a) Part One: What were Karen's conceptions of the model when she first entered the course?}

At the outset of the course, Karen described differentiation as "teaching in a way that responds to students' needs" (interview, 1/27/14). She defined student needs as 
including how they prefer to learn, what they prefer to learn, and the pace at which they prefer to learn. According to Karen, in a differentiated classroom, these needs "drive the decisions you make when you're planning what to teach and how to teach" (interview, 1/27/14).

When I asked Karen to provide a concrete example of what differentiation looks like in action, she offered a scenario in which students create different products to demonstrate their understanding of the water cycle. In this scenario, some students might write a report "if they feel more compelled to explain things through writing" (interview, $1 / 27 / 14$ ), while others might choose to write a poem or draw and explain a diagram. She described, “They're still getting at the objectives you want them to get at, but how they're showing that is based on their needs and their preferences" (interview, 1/27/14).

Karen characterized her initial conceptions of differentiated instruction as very general, saying that she had never taught a fully differentiated lesson before as a result. She explained her goal in taking the course:

I hope to have a better understanding of what differentiation is and isn't, because I think it's a term that's used a lot and thrown around when people say, "Oh, I'm differentiating," but to really know what it is, what it means, what it looks like, what it sounds like, and to be able to start thinking in a way that's more geared toward differentiation. (interview, 1/27/14)

She identified her initial understanding as "more broad than deep" (interview, 1/27/14) in that she believed her definition was correct but that she lacked enough knowledge of its specific components to know which teaching practices would or would not be considered differentiated. 
Karen's description of differentiation at the beginning of the course was consistent with Tomlinson's model of differentiation. While Karen's plans to respond to student learning needs in her future elementary classroom suggested a belief in the importance of differentiation, the primary gap she identified in her conceptions involved lack of knowledge of what the model looks like when enacted in terms of specific teaching practices.

\section{Research Question 1(a) Part Two: How did Karen's conceptions change during the course?}

As the course progressed and she gained new knowledge of differentiated instruction, Karen (a) deepened her holistic understanding of the model, (b) resolved her own misconceptions, (c) developed conceptions of specific model components, (d) recognized the interdependent relationships among model components, and (e) anticipated a role for differentiation in her future practice in light of her beliefs about its potential efficacy.

Deepening holistic understanding. By February, Karen had read three texts about Tomlinson's model of differentiated instruction and attended multiple class meetings. At this point, her evolving understanding of the model focused on her newly developed recognition of its student-centeredness, involving connections between the teacher and individual students. Karen explained, "Differentiated instruction doesn't allow teachers to view their students as just a sea of faces....Instead, it respects students as individuals. It requires teachers to know each of their students and to plan and adjust instruction according to student differences" (reflection, 2/16/14). Karen reflected on the 
importance of appreciating individual student perspectives, particularly in terms of how they responded to the demands of a given task. She explained:

To me it's almost like putting yourself in each of the students' bodies and thinking if I was learning this, how would I best learn it? How would I want to learn it? How could what I'm learning mean something to me? So when you're making decisions about how to teach, it's multi-dimensional. You're not just thinking in one dimension like, this is how I would learn it best, or this is how I think this one student's going to learn it best so I'm going to teach this way for everyone. I think it's trying to look at a set of standards or KUDs through multiple lenses, and it's putting yourself in a lot of different perspectives. (interview, 2/26/14)

She linked the idea of recognizing student perspectives to treating them respectfully, or treating them how they would like to be treated in the classroom, which goes a step beyond how the teacher would want to be treated as a student in the classroom.

Additionally, Karen viewed the Differentiating Instruction course as providing her with knowledge of the model's specific components she felt she lacked when the course began. She described this knowledge as involving the how, where, and when of differentiating:

Up until this course, I've never really known how to account for student differences in my teaching, where in the teaching process I should account for them, and when I should account for them. Differentiation provides a more structured, systematic, and specific way to make decisions that account for student differences within the planning and teaching process. The "how" is changing the process, product, content, or learning environment based on variances in student readiness, interests, and learning profiles. The "where" is in planning, proactively responding to student differences, and in teaching, reactively responding to student differences. The "when" is whenever students would benefit from the content, process, product, or learning environment being fit to student readiness, interest, or learning profile based on results from preassessments. All of these components are being guided by the principles for effective differentiation. (reflection, 2/16/14)

Thus, by February, Karen viewed her newly acquired knowledge of differentiation as reflecting a systematic approach to responding to student differences, both proactively 
and spontaneously, informed by specific information about individual student needs.

Her initial, broad definition of differentiation had been supplemented by specific details about its application in the classroom.

By early April, Karen had had the opportunity to apply this knowledge to planning several differentiated lessons meeting a wide range of student needs. She characterized this experience as demanding, and it refocused her attention on the broader philosophy of the model rather than its specific details. Karen described:

I think differentiation transforms teaching into a craft or art form. In interacting with the concepts of differentiation and applying the concepts to application tasks, I can attest to what a challenge it is to create tasks that stay true to the philosophy of differentiation.... In differentiation, every decision is thoughtful and intentional. In this way, I see that differentiation professionalizes teachers. (reflection, 4/06/14)

This comment was typical of her reflections on differentiation at this point in the course. In applying her knowledge of the principles and practices of differentiation to planning, Karen identified the pervasive intentionality inherent in this approach to instruction. She connected meeting the demands of reaching varied learners to recognizing teaching as a complex and skilled professional undertaking that, when done well, rises to the level of an art. In this way, Karen's focus seemed to shift from the acquisition of specific knowledge of differentiated instruction during the first half of the course to her beliefs about its nature and importance after having the opportunity to apply her knowledge in a planning task.

After the course's conclusion, Karen described her view of differentiation as "more complete" with "a better understanding as to what it looks like to differentiate content, process, and product by readiness, interest, and learning profile" (interview, 5/01/14). When I asked her to review a transcribed copy of the initial definition of 
differentiated instruction she had offered during her January interview, she characterized that definition as "less complete" than her current understanding, rather than inaccurate, although she noted that it did reflect several misconceptions with which she had entered the course.

Resolving misconceptions. A significant portion of Karen's explanations of her evolving conceptions of differentiation during the spring involved two key misconceptions about the model that she identified and corrected at different points in the course. The first centered on the issue of student choice.

Differentiation is not synonymous with choice. In February and March, Karen's discussion of her developing conceptions of differentiation often involved the surprise and challenge she experienced as she came to recognize that in her initial understanding of the model, she had construed differentiation solely as student choice. Before the course, Karen believed that the purpose of differentiation was to give students options that would appeal to their interests and preferred ways of learning, with the goal of increased engagement. In this view, if she provided options, she had successfully differentiated, regardless of learning outcomes. When she looked back on lessons she had planned based on this kind of student choice, she recognized that her activities had not targeted specific learning goals. She explained:

Without clear objectives, I had done nothing more than create engaging activities that led to nowhere. I have come to think of lessons like this as "fake differentiation," because they seem like creative, engaging lessons and great examples of differentiation, but only at surface-level. At closer examination, you can see that...they provide little substance in terms of learning. (reflection, 2/16/14) 
By March, Karen had come to recognize that choice is only one component of differentiation, and that the choices offered to students should align with clear learning goals, reflect quality curriculum, and be based on specific assessment data.

Differentiation is a philosophy. In April and May, Karen recognized a misconception that she characterized as even more significant than the first. She explained that she had been confused during the first few weeks of the course that several class meetings were devoted to the topic of learning environment when she had anticipated that the course would focus only on issues in curriculum and instruction. This was because, before the course, Karen had viewed differentiation to be an "instructional model" (interview, 5/01/14) that encompassed only curriculum, instruction, and assessment. For Karen, the fact that Tomlinson's model also included learning environment and leading students and managing classroom details as two key components made the approach a holistic philosophy of teaching and learning.

Karen felt that the course's structure, in which learning environment was the first of the five non-negotiables of a differentiated classroom to be addressed, helped her to recognize and address this misconception earlier than if curriculum and instruction had been the first topics discussed. Exploring issues in classroom leadership and management a bit later in the course also contributed to her "ongoing process" (reflection, 4/06/14) of conceptualizing differentiation as a philosophy. Karen explained that the significant attention paid to learning environment and leading and managing in the course had "been particularly enlightening in helping me see how the environment and management elements are infused with the curriculum, instruction, and assessment elements" (reflection, 4/06/14). 
Developing conceptions of specific model components. Tomlinson's (2014) model of differentiated instruction encompasses five components: (a) building a supportive learning environment; (b) developing high quality curriculum; (c) integrating ongoing formal and informal assessment to inform instruction; (d) modifying instruction based on learner readiness, interest, or learning profile; and (e) leading students and flexibly managing the classroom. These were the key topics addressed during class meetings of the Differentiating Instruction course and in assigned readings. As Karen encountered information about each of these topics, she considered it in light of her existing knowledge and beliefs about that component of teaching and learning.

Supportive learning environment. While Karen described her beliefs about learning environment as staying the same before and during the course, she also described her knowledge of creating a supportive learning environment as having "expanded a little bit" (interview, 2/26/14). She reported that her beliefs in the importance of having a growth mindset, making connections with individual students, and building a welcoming classroom community existed prior to the course, as well as her belief that the design of a learning environment must be proactive, intentional, and consistently sustained over time. However, during the course, she did learn "more detail" (interview, 2/26/14) about specific strategies for achieving these goals in the classroom.

High quality curriculum. Karen perceived her understanding of high quality curriculum to have "stayed mostly the same" (interview, 2/26/14) before the differentiation course and after the topic was addressed in class meetings and readings; however, she said this was because she had taken a curriculum design course with Carol Tomlinson in a previous semester as a requirement for her add-on endorsement in gifted 
education. Karen reported gaining knowledge in the previous course about writing clear learning goals, aligning other lesson components to those goals, and planning curriculum based on essential understandings.

Karen demonstrated her existing knowledge of these topics during a class meeting in which the topic of high quality curriculum was presented for the first time (observation, 2/04/14). As an introduction to the topic, Tomlinson shared examples of several activities about the planets in our solar system. Without identifying that the activities were not aligned to the same learning goals, Tomlinson asked the class to evaluate the quality of the curriculum the tasks reflected in small group discussion. I circulated among four small groups during this discussion, including Karen's. While none of the other groups identified that the activities did not align to the same learning goals, Karen pointed this fact out to her group. During the ensuing whole class discussion in which a member from several groups shared their conclusions, Karen was the only member of the class who identified this key point (observation, 2/04/14).

Karen's comments during interviews reflected a belief in the importance of high quality curriculum in a differentiated classroom and the role it played in modifying instruction:

I would say it's the foundation of differentiation because, like I said, my preconceived notions were just differentiation as choice. When you get down to it, choice without quality curriculum, choice becomes nothing more than choice.... think that the key is having the high quality curriculum that - to differentiate. Because in the end you can differentiate and differentiate flat curriculum, and it's still gonna be flat. I feel like that's - it's just the foundation. Kind of the starting point. (interview, 2/26/14)

Karen maintained this view of curriculum's foundational role in a system of differentiated instruction throughout the remainder of the semester. 
Ongoing assessment. Karen felt that the Differentiating Instruction course gave her opportunities to practice skills that did not reflect new ideas about assessment but that were applied in a new way when modifying instruction in response to learner variance. She explained:

Making sure that the assessment is purposeful and making sure that the questions you ask are really getting at your learning goal, so just that constant idea of alignment throughout, [These are] not new ideas, maybe, but new skills that I've kind of really been practicing in a way that I hadn't before. Maybe really making sure the questions you're asking on an assessment, like a pre-assessment, are really getting at the Ks or Us or Ds that you plan to differentiate upon. (interview, 4/10/14)

Karen had gained knowledge about aligning assessments to clear learning goals from past experiences, including the previous curriculum design course she had taken with Tomlinson. The Differentiating Instruction course provided opportunities to practice designing assessment questions to elicit data about particular objectives to inform the modification of instruction, reinforcing her belief about the importance of this skill.

Responsive instruction. One of the key areas in which Karen reported gaining new knowledge about differentiating instruction during the course involved specific methods of modifying the content, process, or products students work with based on differences in readiness, interest, and learning profile.

While her initial conception of differentiation before the course focused on interest and learning profile, it expanded during the spring to include student readiness as well, a concept Karen had understood previously but for which she had no name. Karen felt the course provided her with new methods reflecting a more systematic approach to responding to variance in student readiness, which she believed was a key approach to accounting for learner differences. 
Although her beliefs about the usefulness of instruction that responds to student interest or learning profile in harnessing student engagement did not change during the spring, Karen did gain new knowledge about additional student characteristics that may influence learning profile. She reported that, in the past, she had considered learning styles and intelligence preference to be key aspects of students' approaches to learning, but not culture or gender. She therefore had not considered identifying learner needs that might be attributed to those two characteristics and responding to them through some aspect of instruction before the course.

Mid-way through the course, Karen experienced some confusion in distinguishing between teaching strategies that respond to student interest or learning profile. When she thought about a learning activity in which students were able to choose the nature of the product to demonstrate their learning, she had difficulty deciding whether to consider those options as responding to interest or learning profile, feeling that the two seemed similar. She explained:

I think interest maybe refers to topics students are interested in, not so much - and maybe that's how I can kind of distinguish them in my mind...-interest more in topics versus interest in, "Oh, I want to choose create a presentation because I think it's cooler than writing an essay." Interest would be more of the topics they're interested in. Learning preferences would be more of, yeah, they're choosing to create the presentation on a computer versus writing an essay, but it's not necessarily their interest that led them to make that choice. It' $d$ probably be more of their learning style or learning preference. (interview, 4/10/14)

Karen's use of "I think," "maybe," and "probably" in this explanation suggest that, in early April, she was still working to distinguish between the two concepts with total clarity.

By the end of the course, Karen appeared to have fully clarified the idea that differentiation by interest responds to differences in interest in topics, while 
differentiation by learning profile would encompass preferences for demonstrating learning through different formats. When I reminded her of the original example of differentiation she offered during our first interview in January, involving students being given choices among different products to demonstrate their understanding of the water cycle, she said, "That's just an example of differentiating the product by learning profile" (interview, 5/01/14) with no equivocation, and immediately moved on to another topic.

Leading students and managing the classroom. Of the five elements of Tomlinson's (2014) model of differentiation, it was her conceptions of leading students and managing the classroom in which Karen reported the most significant changes during the course.

In February, Karen appeared to conceive of classroom management as part of learning environment, rather than as a discrete element of the model. She described learning environment as including not only the atmosphere and physical set-up of the classroom, but also the routines, procedures, and rules that it involves (interview 2/26/14). By April, however, Karen described learning environment, leading students, and managing the classroom as three related but distinct concepts. Karen defined leading students as:

more about the people you are teaching. It's focused on teaching up to students and not trying to...control what they do and what they say and how they act, whereas leading is more of helping them to become independent learners and independent workers. Helping them know the expectations and monitoring that themselves, and you're just kind of there more as a coach or a tutor to provide support. (interview, 4/10/14)

This conception of leading rather than controlling students raised issues involving what Karen described as "two conflicting ideas of having classroom management" (interview, 4/10/14). The type of classroom management advocated by Tomlinson's (2014) model 
of differentiated instruction challenged her to clarify her own beliefs about the type of management that would best support responsive instruction. According to Karen:

Either you have all of these strict routines and procedures for how the class is run and anchor activities that students know that you do during transition times, or you're totally flexible and don't really have implemented routines. You set expectations, but you don't have a lot of set routines and procedures for how the classroom runs. Now I can see that it's important to have a balance of both, of managing the classroom and then leading your students when managing the classroom. You do have those routines and procedures set in place of how the furniture should be arranged at different times of the day or anchor activities for students to do during transitions. You're not really managing their behaviors, you're more leading them in that way. (interview, 4/10/14)

Karen explained that her student teaching CI had not implemented many routines or transition procedures. As she learned about methods of classroom management suggested by Tomlinson's model of differentiated instruction, Karen could "look back on [her student teaching experiences], knowing this information, [and] see the value" (interview, 4/10/14) of having set expectations for the running of a classroom that still allow for flexibility and student autonomy.

Although Karen saw her natural style of classroom management as being strict since she prefers things "very structured and very routine," the knowledge she gained during the Differentiating Instruction course pushed her out of her "comfort zone" by encouraging a more flexible, less structured approach (interview, 4/10/14). She concluded that she would "pick the things...to stress out over or worry about or create routines and procedures for and what things you can kind of let go" (interview, 4/10/14).

Recognizing relationships among model components. The final aspect of Tomlinson's model of differentiated instruction that played a significant role in the evolution of Karen's conceptions of the philosophy during the course involved the 
interdependent nature of the five elements of a differentiated classroom. By April, Karen had developed an extended metaphor to reflect the connections among them, explaining:

This course has been my first encounter with a philosophy that incorporates all five elements and shows the interdependent relationship among them. The idea that these elements are interdependent is a relatively new concept for me, so I think my understanding will continue to develop throughout the rest of the course and throughout my teaching experiences. I have begun to think of the five elements as the wheels on a grocery cart (though there are only four wheels on a cart, of course). Whenever I go to the grocery store, I always seem to select the cart with one wheel that won't turn easily in a certain direction. I end up fighting with the cart as I push it throughout the store, as it veers off in a certain direction because of the broken wheel. The broken wheel seems to overpower the other wheels and leads the cart in whatever direction it's going. I think this is similar to how a classroom would be if one of the elements is absent or not fully established. Just as the cart needs all four wheels to roll, a differentiated classroom needs all five elements to function successfully. And even if all of the elements are present, a "broken" element can send the classroom off-balance and lead the teacher's efforts astray. (reflection, 4/06/14)

This vivid analogy reflects an understanding of the critical, interrelated functions of each element within a larger system reflected in Tomlinson's model of differentiation. Before the course, Karen had considered some of these elements separately, rather than as an integrated whole, and had not considered how the absence or partial establishment of one element could affect the others.

Karen's Phase 1 model concept map. At the conclusion of the course, during the final interview of Phase 1, Karen completed a concept mapping activity in which I asked participants to create a graphic representation demonstrating how the elements of a differentiated classroom function. I asked participants to include all five elements from Tomlinson's model in their representation, but I invited them to add other elements of a differentiated classroom they believed were missing. Additionally, I asked participants to think aloud as they considered how to structure their representation. Karen's Phase 1 concept map appears in Figure 1. 


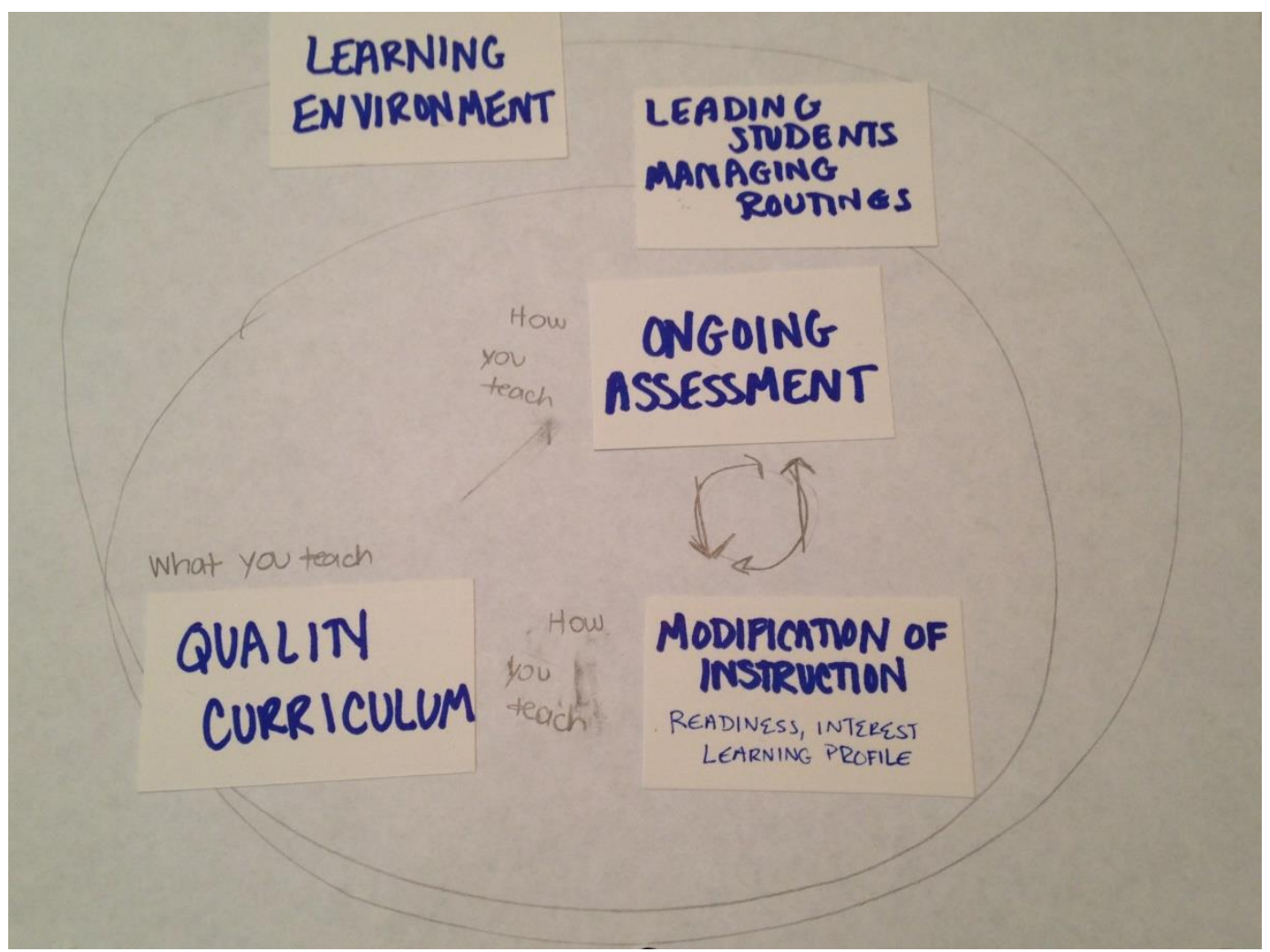

Figure 1. Karen's first concept map illustrating relationships among the five elements of a differentiated classroom. Created during Phase 1 Interview 4 on May 1, 2014.

Karen's representation included two concentric circles. The outer circle represented learning environment. Karen described:

Learning environment would be the biggest circle to encompass everything, because to me, unless you have a positive learning environment, it doesn't matter how good you are with these other four elements. I don't think any of that is really going to happen. (interview, 5/01/14)

The inner circle represented leading students and managing routines. Karen explained that this second element encompassed the remaining three elements, because it had to be in place for the others to "flow smoothly," (interview, 5/01/14). But she also viewed leading and managing as existing within the learning environment because "I don't think you can lead your students without that positive learning environment, where they trust 
you and respect you, and you trust them and respect them," already in place (interview, 5/01/14). She described a teacher's creation of a learning environment as "maybe the warm, fuzzy part of teaching" and the leading and managing element as "the practical part of teaching" (interview, 5/01/14).

Inside the inner circle representing leading students and managing routines, Karen's concept map included what she called "the actual instruction part of it — what you're teaching, how you're teaching, and how you know if what you're teaching is being learned" (interview, 5/01/14). She positioned quality curriculum, modification of instruction, and ongoing assessment in this center portion of the map and drew a small circle made of arrows in between ongoing assessment and modification of instruction (concept map document, 5/01/14). She described a relationship among these three components, with curriculum being the foundational element offering the "place to start" and then a "cyclical process" existing between ongoing assessment and modification of instruction (interview, 5/01/14). She described this cycle as involving pre-assessment, modification of instruction based on the preassessment data, assessing student learning during or after instruction, modifying instruction again based on new assessment data, and so on. She emphasized that successful differentiation depended on this ongoing, cyclical process.

Karen reflected that the knowledge she gained during the Differentiating Instruction course led her to recognize assessment and modification of instruction as being more closely connected than she had realized, since she had previously viewed assessment as existing only for the purpose of "just seeing if the students learned it" rather than to modify instruction based on patterns it revealed (interview, 5/01/14). She 
also came to recognize learning environment and leading students and managing routines as being integrally related to what she termed the "actual instruction" components of teaching for the first time (interview, 5/01/14).

Anticipating future practice in light of beliefs. Karen explained that she believed differentiating instruction would be necessary to support the academic growth of her future students (interview, 4/10/14). She saw modification of instruction based on readiness as being the most important approach for encouraging that growth and hoped that it would play a major role in her practice as a future teacher (interview, 4/10/14). Karen anticipated that two factors would pose the biggest challenges to differentiating effectively in her future practice: time and confidence. She explained:

I think it takes a lot less time to just teach what I think of as in one dimensionkind of just like this is how I would best learn it, so that's how I'm going to teach it. I think with differentiation you have to think about it on a lot of different levels and dimensions and from a lot of different perspectives. (interview, $1 / 27 / 14)$

Karen described herself as someone who needed a lot of time to process complex ideas (interview, 5/01/14). Interestingly, she felt the key challenge of not having enough time to differentiate well in her practice would occur because this approach to instruction requires careful thought on complex issues. Additionally, she believed that she would not differentiate as successfully during her first year in the classroom because she lacked confidence in her ability to do so, but she felt she would gain this confidence with practice (interview, 1/27/14).

Karen's great hope at the conclusion of the Differentiating Instruction course was that, as a new teacher, she would develop the "mindset of differentiation...the normal way I think now, not think something else first and then have to say, well then how does 
that look within differentiation?" (interview, 5/01/14). She hoped that the philosophy of differentiation would become the lens through which she viewed teaching and learning. Karen hoped the philosophy would remain consistently at the forefront of her thinking, and that doing so would allow her to generate ideas about the most effective ways to differentiate in a given situation more quickly. She concluded:

I just hope I can put into practice my philosophy, because it's one thing to be thinking, to have a philosophy, but a different thing to make it connect when you actually put it into practice. And I think it's harder to put some of that into practice than it is to just say that's what you believe. (interview, 5/01/14)

1(a) Summary. At the beginning of the course, Karen's definition of differentiation was consistent with Tomlinson's model, but she construed her understanding as more broad than deep in that she lacked knowledge of specific approaches for putting it into practice. As the course progressed and she gained new knowledge of differentiated instruction, Karen (a) deepened her holistic understanding of the model, (b) resolved her own misconceptions, (c) developed conceptions of specific model components, (d) recognized the interdependent relationships among model components, and (e) anticipated a role for differentiation in her future practice in light of her beliefs about its potential efficacy. She ended the course believing differentiated instruction would play a role in her future practice, hoping that it would become the lens through which she instinctively viewed teaching and learning.

\section{Research Question 1(b): How did factors external to the course relate to Karen's} conceptions of the model?

As Karen made meaning of the philosophy and practice of differentiated instruction over the course of the spring semester, she identified previous experiences that played a role in her developing knowledge and beliefs about the model. These 
factors involved varied experiences in her teacher education program and her own background.

Personal background factors. As Karen discussed differentiation during interviews and reflections, she referenced a number of aspects of her background that she saw as related to her evolving conceptions of the model. These factors included her existing beliefs about teaching and learning that she brought to the course, her own educational experiences in K-12 settings, and other relevant personal characteristics.

Prior beliefs about teaching and learning. Karen began the Differentiating Instruction course with a general philosophy of teaching and learning that was specific and well-defined. She described her existing beliefs as reflecting a student-first approach in which the teacher strives to teach in a way that encourages students to learn because learning feels enjoyable, relevant, and connected to their own lives. For Karen, doing this effectively involves recognizing that students are "little human beings with unique interests and talents... and little things that make them special—-that they are individuals" (interview, 1/27/14). Karen viewed getting to know students as individuals as necessary for understanding who they are as learners and adapting the classroom to meet their varied needs. She believed that, for this to occur, students need to feel welcome, trusting, and supported by the classroom community, and that all students in a heterogeneous classroom should be challenged to work as problem-solvers and creators (interview, $1 / 27 / 14)$.

After acquiring new knowledge about differentiation throughout the course, Karen noted that significant overlap existed between her personal philosophy of teaching and learning that she brought to the course and the philosophy reflected in Tomlinson's 
model. In particular, she found strong alignment between differentiation's philosophy and her beliefs that all students are capable of learning and that a teacher should respond to individual student needs in a systematic way. Karen explained how she made meaning of that alignment:

I had the underlying beliefs, and since I've always wanted to be a teacher, I've had a long time to form my beliefs about teaching and why I wanted to teach and what about teaching is so appealing to me. But I would say the course helped me define those beliefs and then reaffirmed what I had been thinking. (interview, $5 / 01 / 14)$

Karen's experiences in the Differentiating Instruction course gave her opportunities to further define her own beliefs about teaching and learning, and in turn reinforced her existing beliefs due to the alignment between them and the philosophy reflected by the model. As she considered the process of examining the alignment between her own beliefs and differentiation's philosophy throughout the course, she concluded that entering the course with her existing philosophy enhanced her developing conceptions of the model, saying that her existing beliefs ended up forming the foundation for understanding differentiation.

Prior educational experiences. As an elementary student, Karen had been identified as gifted and was taught in homogeneous classes for gifted students. She attended middle and high schools that were tracked and took mostly advanced and honors courses as a secondary student. Karen could not recall having been a student in any classrooms that she would describe as differentiated, explaining that her classes instead seemed to involve "a one-size-fits-all curriculum where all students are doing the same thing all of the time. It's not really tailored to the student. It's more tailored to however the teacher wants to teach it" (interview, 4/10/14). While she felt that this type of 
instruction "most of the time worked fine" (interview, 4/10/14) for her because she was a successful student who worked hard and was good at memorizing facts, she believed that students who were not in advanced and honors courses may have struggled more with instruction that was not responsive to their individual needs.

Interestingly, Karen viewed this lack of differentiation in her own educational experiences as a factor that enhanced rather than detracted from her understanding of the model. She explained:

I saw what it didn't look like. It was more of a non-example of differentiation, so seeing a non-example helps you know what it is....[K]nowing now what differentiation is and how it looks and seeing that was not present kind of helped me I guess see there was a need for it and something was missing from that instruction, even though it worked well or fine for me. (interview, 12/08/14)

In particular, Karen recalled an experience in a non-honors physics course in which she had no trouble memorizing formulas and applying them, while a number of her peers struggled to do so. In retrospect, she believed that her peers would have benefitted from a different approach to instruction. Karen came to see personal experiences in nondifferentiated classrooms like this as opportunities "to see what was lacking" (interview, $12 / 08 / 14)$. In this way, her prior educational experiences informed her belief in the importance of differentiation, rather than providing knowledge of how differentiation might be implemented in a $\mathrm{K}-12$ setting.

Personal characteristics. In addition to her existing philosophy of teaching and learning and her own K-12 educational experiences, Karen saw connections between some of her personality traits and her conceptions of differentiation. First, Karen characterized herself as a caring person, making her a teacher who cares about her students and has a "genuine interest to learn about them as individuals" (reflection 
2/02/14). She believed a teacher should be intentional in taking the time to do this and work to connect with them "to understand or relate to their struggles and experiences" (reflection, 2/02/14). While she was concerned that connecting with students from homelives different from her own "more privileged life growing up" (reflection, 2/02/14) might be more challenging, she believed it was still possible by communicating genuine interest in students' lives and appreciating differences.

Karen also described herself as a sensitive person (interview, 4/10/14). She connected this personal trait to experiences from her own family background and to her conceptions of differentiated instruction. Karen is the middle of three girls in her family, and during the course, she recognized some aspects of the principles of differentiation in how she had been raised by her father. She explained:

I think of how he has the same values and the same lessons that he wants to teach us, but how he does it is a lot based on our age and experiences, and also our personalities, because my older sister can take a lot more honesty and brutal feedback, whereas I'm more sensitive, and so is my younger sister. He changes his approach with how he comes to us with different issues. (interview, 4/10/14)

Recognizing a parallel between her father's approach to teaching his daughters about issues such as financial responsibility and a differentiated approach to classroom instruction supported Karen's understanding of differentiation by making it more concrete through a connection to her own life.

Mindset. Last, Karen saw a significant relationship between the personal characteristic of her mindset and her developing conceptions of differentiation. While she characterized herself as having a growth mindset, she felt that it was easier to maintain it with her students than with herself and felt she might face a challenge in keeping it as a new teacher. Karen explained: 
I don't want a fixed mindset to discourage me from trying new methods and strategies for fear of failure. I don't want my inexperience to become an excuse for teaching flat curriculum and using surface-level activities to build a learning environment....Instead of focusing on my mistakes, I will focus on what I can learn from those experiences and what I can change to make my plans more successful in the future. I will view my development as an educator along a continuum versus a path with a specific "finish line," understanding that there is always more to learn and more ways to grow. (reflection, 2/02/14)

Karen considered a teacher's growth mindset toward herself and toward her students to be critical for effective differentiation. She hoped that she would enter the classroom in the fall without a pressing fear of failure and with a patient attitude toward her own growth. She believed this would be important, not only for setting and meeting high expectations for her own professional performance, but also so that she could "model how I expect my students to think about themselves and their classmates" (reflection, 2/02/14).

Teacher education program factors. In many reflections and in every interview, Karen noted the significant relationships between her evolving conceptions of differentiated instruction and her experiences from the previous three semesters in her teacher education program, including her PG/MT coursework in the elementary education and gifted education endorsement programs and her numerous field placements.

Prior coursework. As a student in her fourth and final semester of the PG/MT program in spring 2014, Karen had completed two semesters of coursework and one semester of full-time student teaching before the Differentiating Instruction course began.

Prior coursework content. At the outset of the study in January, Karen attributed her existing knowledge of differentiation to prior experiences in her teacher education program: "We touched on it briefly in my instruction class last year...And then you hear it 
thrown around a lot—in the school I was student teaching in and in other courses, it will kind of weave its way in there" (interview, 1/27/14). She also recalled that one assigned reading for her general methods of instruction course had been a brief article about differentiation written by Tomlinson, although Karen could not recall the nature of the class discussion about the reading or her reaction to it at the time.

In late February, Karen characterized the treatment of differentiation in her previous coursework in more detail:

I think [this course has] given me a more systematic way to know how to account for student readiness. Because we're always told in classes, "Adjust your instruction for your students." No one's really told us more of a systematic, structured way of how to do that.... think that's really helped for me, just giving me more structure and more of a system to go about doing that than just generally saying, "Account for student differences." (interview, 2/26/14)

Additionally, in an April reflection on how her conceptions of differentiation had changed during the spring, Karen noted, "I have also come to know differentiation as a philosophy, rather than a curriculum model. This course has been my first encounter with a philosophy that incorporates all five elements and shows the interdependent relationship among them" (reflection, 4/06/14).

The nature of these comments, which Karen made at different points during the semester, suggests that as the course progressed, she recognized in different ways how she had and had not been exposed to the principles and practices of differentiation in previous coursework. While at the beginning of the course she viewed differentiation as having been woven throughout her coursework, in February, she related that her previous coursework had underscored the importance of accounting for student differences but had not presented a systematic approach for doing so. Karen's April comments indicate that she reached two additional conclusions during the course: First, that the philosophy of 
differentiation had not been presented in other courses, and second, that her previous coursework had not emphasized the interdependent nature of the classroom components involved in teaching and learning. Karen explained, "In my previous education courses, the five elements...have all been addressed at some point, mainly as separate elements....There was some mention of learning environment and classroom management, but these elements were not infused with discussion of instruction, curriculum, and assessment" (reflection, 4/06/14).

Prior coursework instruction. Karen also noted that, although she recognized numerous ways in which differentiated instruction had been modeled by Tomlinson during the Differentiated Instruction course (interview, 5/01/14), she had not seen the instructional approach modeled in her other coursework (interview, 4/10/14). She explained that this was an issue she had thought about throughout her program and that she believed the issue stemmed from her being enrolled in the PG/MT program (interview, 4/10/14).

Karen felt that the students in the PG/MT program brought diverse experiences to the field of teaching, with some of her peers having just graduated from undergraduate programs, others having switched fields after a long career, some having experience working with children, and others never having spent time in a classroom. She also noted that the professional and life experiences of PGMTs were often different from those of the BAMTs in her classes. In some courses, Karen felt that the instruction she experienced had not accounted for these differences in significant ways and did not reflect an understanding of individual students' varied backgrounds and needs (reflection, 3/23/14; interview, 4/10/14). Explained Karen, "Because of the wide range in student 
[readiness] variance, I feel I would've learned more from my classes if the curriculum and instruction were adjusted to account for these variances," with all students being provided "appropriate challenge and support" in light of their level of background knowledge and experience (reflection, 3/23/14).

Field experience. During the year and a half before the study began, Karen completed five field experiences. In her first year of the PG/MT program, she spent several hours per week in her field placements, with two different placements each semester for a total of four placements that year. Each semester of the first year, she observed or provided individual students with one-on-one support in one placement, while the other placement involved teaching a handful of short lessons. She was placed in kindergarten, first, third, and fourth grade classrooms for these experiences. Karen then student taught fulltime in the fall of her second year at the second grade level.

Initial placements. Karen believed that the experiences she had in the four field placements she completed during her first year of coursework were not significantly related to her conceptions of differentiated instruction (interview, 2/26/14). She said she "barely" remembered her experiences from those placements and that "It was almost too short of hours to even get to know the kids, to get to know the teacher....you go in and teach for isolated lessons and then you're out of there" (interview, 2/26/14). Karen said she never made connections between the ideas she was considering in the Differentiating Instruction course and those field experiences. She could not recall seeing any examples of a differentiated approach to instruction in those four settings. Instead, it was Karen's student teaching experience that she continuously recalled as she made meaning of the concepts involved in Tomlinson's model of differentiation. 
Student teaching. Karen characterized her student teaching experience as "really positive" and the best learning experience she had had in graduate school to date (interview, 2/26/14). She described her cooperating instructor, or CI, as generally attuned to student needs. While her CI did not use the term differentiation when discussing his instruction with Karen, his approach to teaching was focused on student autonomy and comfort in the classroom, and he frequently modified his instruction based on student interest and learning profile.

Karen explained that her CI often gave students choices in what or how they learned, such as choosing an aspect of the content to learn about in greater depth or whether to work alone or with a partner. One example of this involved a series of longterm research projects. Every Wednesday, students researched a topic of their choice and recorded information about it. Ultimately, they presented their findings to the class through a presentation format of their choice. In many cases, however, Karen felt that the choices her CI gave to students were not tied to a set of clear learning goals. With the Wednesday research projects, which the CI intended to address standards involving reading non-fiction text, Karen felt there was not enough clarity surrounding what students should know, understand, and be able to do for the teachers or, more importantly, for the students. She felt that, as a result, the products students created did not always adequately address the standards (interviews, 1/27/14, 2/26/14). As the course progressed, Karen also came to view these choice-based lessons as lacking other aspects of high quality curriculum, such as being grounded in essential understandings, and as not being adequately informed by assessment data (reflection, 3/23/14). Concluded Karen, "Without being grounded in those components of instruction, the 
choices only benefitted students by providing different options" (reflection, 3/23/14). It seems likely that Karen's initial misconceptions about differentiation being synonymous with student choice were influenced by this aspect of her CI's instruction.

Karen described her CI's classroom management style as extremely flexible, saying he "let the students sit wherever, whenever....I felt that was too relaxed for me" (interview, 2/26/12). Her CI lacked routines and structured procedures for transitions (interview, 4/10/14), which meant that the class "would often waste valuable class time trying to gather the class and ensure they were ready to learn again" (interview, 3/23/14). She connected this relaxed approach to management to the CI's belief in creating a learning environment that would offer students autonomy in the running of their classroom and would be physically comfortable for them. She concluded, "He's very big on independence and trusting that students can be independent" (interview, 2/26/14). It seems Karen's efforts to determine the right balance of flexibility and structure in classroom management during the Differentiating Instruction course may have been influenced by observing a classroom during student teaching that she considered to be managed too flexibly.

Karen could not recall seeing meaningful examples of modification of instruction by readiness during student teaching other than strategies used during language arts instruction such as Word Study. She felt that the absence of differentiation in response to student readiness was particularly significant in math class:

Though the students were grouped into different math classes based on preassessment data, there was still a wide range of variances in student readiness in regards to math. Because of these variances, students would have benefitted from having instruction differentiated according to their readiness. I often felt it was a struggle to provide adequate challenge and support for all students when they completed the same activities, and differentiating by readiness would've allowed 
students to engage in learning opportunities pitched at the appropriate level of challenge for them. (reflection, 3/23/14)

Karen felt that seeing more examples of responsive instruction, particularly based on readiness, during student teaching would have supported her in making meaning of differentiation during the course.

As Karen looked back on her own teaching practice during student teaching, she reflected that it often did not incorporate the principles of differentiation, particularly in the areas of curriculum and instruction. First, she recalled that the lessons she presented were not grounded in essential understandings and ideas that were likely to engage students. Karen explained, “As far as my practice goes and actually teaching, I had abandoned the high quality curriculum thing when — in student teaching, because I was so focused on just what the students have to know, understand, and do for the SOLs" (interview, 2/26/14). Once she took over the responsibilities of leading the classroom from her CI, Karen's main focus became addressing mandated standards, and her knowledge of high quality curriculum, which she attributed to taking Tomlinson's curriculum course before student teaching, no longer informed her planning.

Second, Karen felt that meaningful modification of instruction was lacking. The lesson plan template she was required to use by her program included a section for describing strategies for English language learners used during the lesson. Because Karen had no English language learners in her class, her university supervisor instructed her to list strategies for differentiation in that section of the template instead and provided her with examples of what to list, such as creating opportunities for students to listen to an audio version of a story or having students work with the teacher in small groups (interview, 4/10/14). Karen explained: 
What we would list under those things isn't really differentiation, but it's kind of what people wanted to see in our lesson plans. So for one example, we...would be providing visuals through the book or letting students draw the setting instead of just having to write about it, that kind of thing....Again, I think differentiation is the wrong word to use for these things. Strategies for support might be a better term for it now that I'm kind of getting into it [the Differentiating Instruction course], but we called it differentiation then. (interview, 1/27/14)

During a later interview, Karen expanded on planning for differentiation during student teaching:

I think what I did was just tapping the surface of differentiation. It was thinking about it, but it wasn't really thinking about specific students in mind, and it wasn't thinking about specific content pieces. I think...it skimmed the surface of differentiation at best....I didn't understand how to [differentiate]. That was one thing I really - they always say account for student differences, but the big question is: How? I never really knew how to account for it—what student differences we're talking about and then how to account for it. (interview, 4/10/14)

Immediately following this comment, when I asked Karen whether she had ever asked her supervisor how to account for student differences in a particular lesson, she answered:

No. Once I got the OK ratings on my rubric [used by the supervisor to evaluate the lesson plan], I just moved on....I mean, it was stuff in the lesson I was already doing anyway. It wasn't like I went back and said, "How can I differentiate this lesson?" It was like, "What can I pull out to make it appear like differentiation?" (interview, 4/10/14)

Karen's experience working with a university supervisor on differentiation did not enhance her understanding of the model. To begin with, Karen felt she lacked a deep understanding of how to identify student differences and account for them. She also had no incentive to go beyond "skimming the surface" of differentiation and therefore did not plan lessons intentionally around student differences. Instead, differentiation became a kind of afterthought for Karen — a box on a lesson plan template to be filled in, so she sought to identify aspects of lessons she had already planned that would "appear like 
differentiation" to meet what she construed as the limited expectations of what her supervisor "wanted to see."

Reactions to teacher education program factors. Karen reflected on the relationship between her previous experiences in her teacher education program and the conceptions of differentiated instruction she developed in the course during interviews in May 2014 and December 2014.

Reactions at course conclusion. Just after completing the Differentiating Instruction course, Karen characterized her experiences in the course as "retraining" her thinking, since she was "learning different things in different ways" about teaching than she had learned in her previous coursework (interview, 5/01/14). The two key areas in which Karen felt her thinking was "retrained" in the differentiation course were (a) writing clear learning goals involving essential understandings and aligning assessment and instruction to them, and (b) recognizing the connections among classroom learning environment, leading students and managing classroom routines, and instructional issues (interview, 5/01/14). Of these two areas, Karen believed the first was the more significant in terms of detracting from her understanding of differentiation. She explained that, in previous courses:

We talked about writing learning goals, but not in the same way that we write them [in this course], not with the clarity and not with leading students to deeper understandings....And there was not the necessity of clarity and alignment with your learning goals, assessment, and learning activities differentiation shows. I feel that's one of the things that's been the most difficult for me [in this course]. (interview, 5/01/14)

She also felt that not seeing many examples of effective differentiation in elementary school field placements detracted from her understanding of the model. 
Karen concluded that learning about differentiation in depth from the beginning of her teacher education experiences would have better supported her developing conceptions of the model. She explained:

That would have enhanced my understanding. I think it would have just started me off on a totally different foot, thinking about my curriculum and my instruction, and thinking about what differentiation was. But then I think I did have a better appreciation and understanding of it after being in a student teaching environment too, and having that when you're really kind of immersed in the classroom [also would have been helpful], so it's hard to say, but I wish we would have been exposed to it earlier at least. (interview, 5/01/14)

Looking back on her teacher education experiences, Karen believed that learning about and applying Tomlinson's model of differentiation, including specifics about how to identify key student differences and account for them meaningfully through instruction, during each phase of early coursework, student teaching, and coursework following student teaching would have been beneficial to her understanding.

Subsequent reactions. In December 2014, Karen had been leading her own classroom for one semester. During our December interview, I asked her to reflect on the relationship between her conceptions of differentiated instruction and many factors involving her personal characteristics and teacher education experiences that she had mentioned in previous interviews and reflections (see Appendix H). Karen considered each of these factors and classified them as enhancing the development of her conceptions of differentiation, detracting from her developing conceptions, or playing no role in her developing conceptions, or she indicated that she was uncertain as to whether it was related to her developing conceptions. After she had explained her responses to all factors, I asked Karen to consider each factor she had characterized as playing a role in the development of her conceptions, either by enhancing or detracting from it, and to 
identify the three factors that had played the most significant roles. She explained that, of all the factors related to her evolving conceptions of differentiation during the course, the three most significant factors involved her experiences in the differentiation class itself, her previous Curry coursework, and her field placements (interview, 12/08/14). She related that she believed what she learned in Tomlinson's course had the most influence on her conceptions of differentiation in that it greatly enhanced her understanding of the model's principles and practices.

The second most important factor she identified as related to her developing conceptions of differentiation involved her previous coursework, which she believed detracted from her knowledge and beliefs about differentiation. She explained:

Because I didn't have any exposure to it in my other courses, it detracted from my understanding at that time.... because that made up the majority of my time in grad school- three semesters of not having differentiation versus one semester having it. (interview, 12/08/14)

Because Karen viewed her experiences in the Differentiating Instruction course as "retraining" her thinking about key aspects of teaching and learning (interview, 5/01/14), she came to view her time in graduate school as divided into two segments. During the longer segment, reflecting three-fourths of her time in her teacher education program, she did not meaningfully understand differentiation. During the shorter segment, reflecting one-fourth of her time in the program, she did. She therefore viewed the fact that the majority of her time in graduate school was spent in the phase when she did not understand differentiation as a key detractor from her evolving knowledge and beliefs about the model.

The third most important factor she identified as related to her developing conceptions of differentiation involved her field experience, which she felt had not 
included enough examples of differentiation. Karen believed her field placements had provided:

plenty of examples of how it is not implemented or how it doesn't work, but I didn't see any of how it would work. That's what I'm struggling with in my math instruction all year is seeing how it does work. What does it look like practically speaking when you have such a wide range of students? (interview, 12/08/14)

It should be noted that it is possible preservice teachers like Karen do observe the use of differentiated strategies, structures, or approaches in their field placements which CIs do not explicitly describe as "differentiation" or talk about in depth with student teachers. Preservice teachers may encounter such examples but not have adequate knowledge or experience to recognize them as forms of differentiation. It should also be noted that Karen made this statement half a year after the conclusion of the course and more than three months into her teaching career. During our May interview, Karen mentioned briefly that it would have been helpful to have seen more examples of differentiation during her field placements but emphasized that she considered the misalignment she perceived between the differentiating instruction course and her other coursework as much more significant in terms of detracting from her developing conceptions of the model (interview, 5/01/14). By December, Karen seemed to view this aspect of her field experience as much more consequential in terms of detracting from her understanding of differentiation, largely because, once she began trying to modify instruction in her own teaching practice, she found she did not have a clear enough sense of what it should "look like" (interview, 12/08/14).

1(b) Summary. Throughout the course, as Karen made meaning of the philosophy and practice of differentiated instruction, she identified relationships between her developing knowledge and beliefs about the model and her previous experiences. 
These experiences involved her teacher education program and her own background. These personal background factors involved her existing philosophy of teaching and learning, her prior K-12 educational experiences, and personal characteristics. The overlap between Karen's personal philosophy of teaching and learning focused on meeting individual student needs and the philosophy of differentiation enhanced her developing conceptions of the model. Her own educational experiences in K-12 settings that did not include much differentiation informed her belief in its importance, as she recognized problems created by its absence. The way in which her father taught life lessons to her and her sisters in different ways in light of their needs offered Karen a model of differentiation from her own life experience, and she hoped her growth mindset would support setting high expectations for herself as a teacher and modeling a growth mindset for her students.

The second category of factors that had a relationship to Karen's developing conceptions of the model involved experiences in her teacher education program, both coursework and field placements. Although differentiation had been mentioned in some of Karen's previous coursework, it had not been modeled by course instructors. Karen characterized her experiences in the Differentiating Instruction course as retraining her thinking about responding to student differences in light of what she had learned in other courses, and she viewed this as a detraction from her understanding. Although Karen's student teaching experiences provided her with models of modifying instruction by interest and learning profile, these instances did not reflect the principles of high quality curriculum. Her cooperating instructor did not modify instruction by readiness. Karen viewed this as a factor that detracted from her conceptions of differentiation, since once 
she began trying to modify instruction in her own teaching practice, she found she did not have a clear enough understanding of what it should look like.

\section{Karen's Phase 2 Experiences}

In March 2014, Karen was hired for the 2014-2015 academic year to teach kindergarten at Newland Academy. Newland is a private school in a mid-Atlantic state with around 150 students in preschool through eighth grade programs. ${ }^{5}$ While Newland generally attracts children from middle and upper-class families, it also offers financial aid for qualified students. The school was founded by its current principal, Beth. Newland Academy's stated school philosophy centers on differentiated instruction, and a number of its grade levels other than kindergarten have multi-age classrooms that span two grades. Although most grade levels have multiple sections, Karen was hired as the school's only kindergarten teacher. Her class has 17 students, with nine boys and eight girls. Fourteen of the students are Caucasian. One is African-American, and two are of mixed racial heritage. At the beginning of the school year, all of the students were five years old. All of her students had attended preschool the previous year, with four of them having gone to preschool at Newland. Karen has one teaching assistant, Susan, who works with her class most of the day. Susan has worked as an elementary school teaching assistant for over five years but is not a licensed teacher.

\section{Research Question 2(a): What were Karen's conceptions of the model as she first entered the classroom, and how did her conceptions change during the fall, if at all?}

At the start of the school year, Karen characterized her conception of differentiation as remaining unchanged from the conclusion of the Differentiating Instruction course except for its being "reinforced," a word she used multiple times

\footnotetext{
${ }^{5}$ Student demographic information for Newland Academy is not publicly available.
} 
during the interview $(9 / 01 / 14)$. Speaking to me after meeting with her students' parents but before the first day of school, she explained:

I would say it's been reinforced, like especially thinking how I'll have one who apparently reads on a third grade level and some who are not reading. I was just telling my parents about this. I mean, you're expected to teach to both them and to be responsible for their growth, so that's reinforced why we need differentiation, and then seeing how different my kids are and going over their different strengths and areas for improvement [with their parents]. It's just been more reinforced. (interview, 9/01/14)

Thus, Karen's experiences as she was just beginning to learn about her students' differences strengthened her existing beliefs about the need for differentiation to support the growth of each student. Before the first day of school, Karen was already aware of broad variance in student readiness with reading skills, anticipating a significant need for modification of instruction in that area.

At the end of Karen's first month in the classroom, I asked her, now that she was getting to know her 17 students, whether her knowledge and beliefs about differentiation had changed at all since the start of the school year. Again, she responded by using the term "reinforced" multiple times (interview, 9/29/14):

I wouldn't say it's changed, but...just to see the wide range, it's like, oh my gosh! It just reinforces the need for differentiation. And I knew that before [the school year began], but actually having kids to say, all right, my highest kids are here, and my lowest ones are here. How in the world would that work to be teaching them the same thing? It just reinforces I think the need for it. (interview, 9/29/14)

During the first month of school, this "need for differentiation" manifested itself primarily in terms of differences in student readiness, which Karen decided to make her priority: "For me the most, what I need to do first is readiness, because they need to be challenged. They need to be engaged in what we're doing before I can kind of bring other things in" (interview, 9/29/14). Karen anticipated modifying instruction by interest 
and learning profile at a later date, seeing them as being of secondary importance compared to addressing students' readiness needs.

Karen's Phase 2 concept map. In October, Karen repeated the concept map activity she had completed during our final Phase 1 interview in May. I asked her to create a representation of the relationships among the five elements of a differentiated classroom and to think aloud as she created it. Although Karen said she could not remember her representation from May and seemed frustrated at being unable to recall her initial version of the concept map (see Figure 1), her second version created in October (see Figure 2) was almost identical, as were her explanations of the relationships among the elements (interview, 10/16/14).

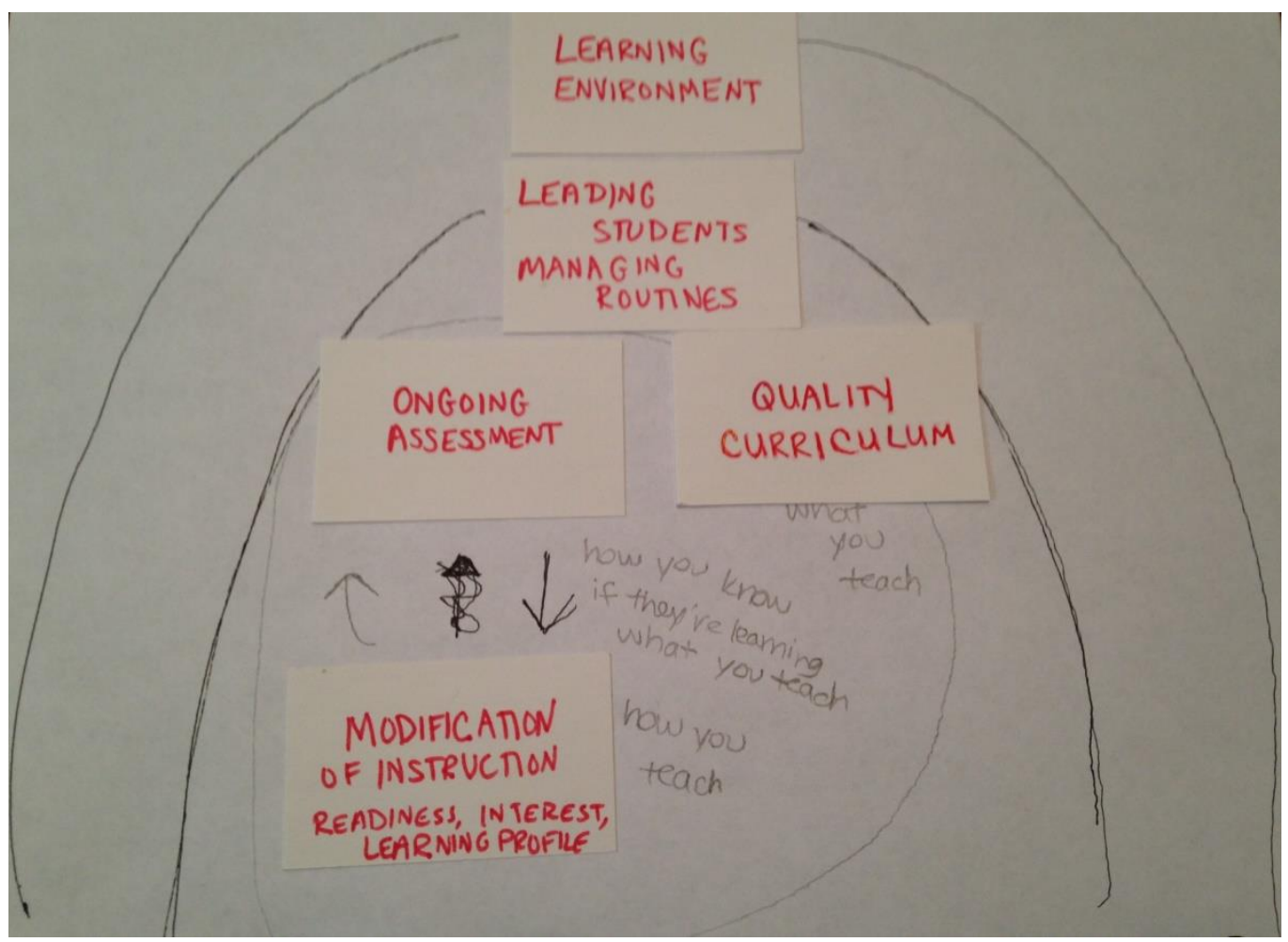

Figure 2. Karen's second concept map illustrating relationships among the five elements of a differentiated classroom. Created during Phase 2 Interview 2 on October 16, 2014. 
I then asked Karen to review her original concept map. When I asked her why she thought there were no significant differences between the version she created as a preservice teacher and the version she created as a first year teacher, she explained:

It doesn't surprise me too much. I think taking differentiation after student teaching had a major role to play in that, because before you have any students to picture in your mind, you don't see how important learning environment and leading and managing are, I don't think. Like I think we spend a lot of time thinking about curriculum and instruction in our graduate courses, but not so much about the learning environment or leading and managing, so I don't think you get the full effect of that until you see it happening. (interview, 10/16/14)

Karen was not surprised by the fact that her understanding of the relationships among the elements of a differentiated classroom had not changed as a first year teacher. Her comments suggested she believed shifts in that understanding had not occurred because she had classroom experience from student teaching in mind when she learned about differentiation in the course. She could therefore visualize how the principles of differentiation did or could have played out in a particular classroom with a particular group of students while she was learning about those principles. Karen did not feel that the experience of leading her own classroom had caused her to develop a new or deeper understanding of the interdependence of those elements.

2(a) Summary. While Karen did not view her conceptions of differentiation as changing from the spring to the fall, she noted that her understanding of the need for it was strongly reinforced as she got to know the individual students in her class. In this way, her beliefs about the importance of differentiation seemed to be fortified as a new teacher. Karen decided to make the modification of instruction by readiness her priority during the first year to ensure her students were adequately challenged. She attributed the fact that her understanding of the relationships among the elements of a differentiated 
classroom had not shifted since the spring to having taken the differentiation course after she had already led a classroom as a student teacher, giving her a specific group of students to envision as she considered the model's principles and practices.

\section{Research Question 2(b): What was the relationship between Karen's conceptions of the model and her teaching practice?}

- "The reality of actually putting it into practice is difficult" (interview, 9/29/14).

- 'I'm working on it, and it's not where I want to be by the end, but it's a work in progress" (interview, 10/16/14).

- "It's just more difficult to put into practice than I had anticipated" (interview, 11/17/14).

- "It's still always a constant challenge" (interview, 12/08/14).

These statements, which Karen made throughout the fall of her first year in the classroom, were typical of her comments about the relationship between her knowledge and beliefs about differentiation and her teaching practice. Although Karen implemented many practices reflecting key principles related to all of the non-negotiable elements of a differentiated classroom, she was resolutely determined to continue to improve the ways in which she identified and responded to diverse student needs in her classroom.

During the first four to six weeks of school, Karen's focus was on establishing a supportive learning environment and classroom management procedures. Her focus then shifted to issues involving curriculum, instruction, and assessment, especially those that involved the modification of instruction by student readiness.

Learning environment. Karen described the aim of the classroom learning 
environment she established for students as to "help them, first of all, feel comfortable being at school, and then building that environment where they feel safe to take risks" in their learning (interview, 9/01/14). During the fall, she succeeded in creating a learning environment that fully aligned with her beliefs about the kind of positive climate necessary for effective differentiated instruction to occur in a classroom.

Physical environment. Before the school year began, Karen's main focus was setting up her classroom space. Karen spent several weeks at the end of the summer arranging her classroom. She made decisions about these arrangements by "thinking about how it would look to a student coming in, how to make them feel comfortable, how to make it look warm and welcoming" (interview, 9/01/14).

Karen's kindergarten classroom is a large space, approximately 35 feet long by 20 feet wide. It is located on the second floor and takes up the entire length of the school building. The furniture arrangement divides the room into six sections: (a) the "carpet" area in front of the white board, where students sit on the floor for whole-class activities; (b) the "library," which has plush chairs and a swing hanging from the ceiling; (c) a teacher's area with a kidney-shaped table and chairs for Karen to meet with students for group work, as well as shelves with many of her supplies; (d) a central area with five circular tables that seat four students; (e) a corner of the room sectioned off from the central area by book cases with a sixth table and chairs where the teaching assistant can meet with students for group work; and (f) a corner of the room with student cubbies and a seating area with a plush chair and fluffy pillows on the floor.

The "carpet," "library," and teacher's area are at one end of the 35 foot long classroom, while the corners with the sixth table and with the seating area are at the 
opposite end. This configuration allowed students to spread throughout the spacious room for independent reading time (observation, 9/29/14, 11/17/14), work at four stations in different locations (observation, 10/16/14, 11/17/14), or be led by the teacher or teaching assistant for group work without many noise problems. The walls of the classroom are painted bright blue, and colorful paper lanterns hang from the ceiling above the teacher's area and next to the student cubbies. A tree is painted on the wall in the library, and three paper mache leaves over a foot long hang from the ceiling next to it. There are six windows in the classroom on three of the four walls, letting lots of natural light into the space. The furniture is low to the ground so that young children can use it comfortably, and the white board and posters in the carpet area are hung on the wall around a foot and a half above the floor so that students can see them easily. The effect of Karen's thoughtful classroom design was to make the space inviting for kindergarteners. Karen described herself as "very lucky" (interview, 11/17/14) to have such a large room with lots of furniture of different configurations and felt that this supported her implementation of differentiation since it allowed different groups to spread out easily (interview, 12/08/14).

Non-physical environment. Karen also attended to establishing a positive climate in the classroom and responding to students' affective needs. Throughout every observation, Karen spoke to students in a warm, calm voice and smiled frequently (observations, 9/29/14, 10/16/14, 11/17/14, 12/08/14). She never spoke in an angry tone or raised her voice at any point, including instances when she was re-directing student behavior (observations, 9/29/14, 10/16/14, 11/17/14, 12/08/14). Additionally, during all four of my classroom visits, I observed multiple interactions between Karen and her 
students that suggested she was creating a learning environment in which she made personal connections with individual students, built a community where every student felt welcome, and encouraged students to develop a growth mindset.

Connections. Karen designed opportunities intentionally to get to know her students as individuals. These interactions took place during formal instruction time and during less structured periods. Rather than planning for the next activity or leaving the students with Susan to interact with other teachers, Karen generally spent snack time and lunch time with students. At snack time, she walked from table to table talking to her students, and at lunch time, she sat down to eat her own lunch at a table with students. During this time, she spoke with students about their lives and interests outside of school.

During one observation, I listened to several exchanges among Karen and a few of her students at snack time (observation, 11/17/14). Earlier that day, Lucy had been dropped off by her father several hours late because her mother had had a baby boy. Lucy frowned as her father told the class this news and continued to frown after he left and she completed the morning learning activities. As soon as snack time began, Karen approached Lucy to ask when her brother was born. Still frowning, Lucy said she was not sure because she had been staying at her grandmother's house. Karen asked Lucy if she had gotten to hold the baby. Lucy said no, that she had not seen the baby yet. During this exchange, Lucy looked down at the table, not making eye contact with Karen, and spoke in a monotone. At this point, Karen leaned over so that her face was close to Lucy's. As Lucy looked up, Karen smiled at Lucy and said that her baby sister had also been born when she was around Lucy's age, and that because she was sick when the baby was born, she had to wait a long time before she could hold the baby. Lucy did not reply, 
but she nodded slightly and her frown softened (observation, 11/17/14).

This exchange was typical of the personal connections Karen worked to create with her students. Although these interactions usually did not involve Karen sharing a personal anecdote, they all reflected her attempts to form a personal relationship with each student by understanding who they were and the experiences that shaped their lives.

Community. Karen communicated to students that her classroom was a space in which all members were valued and respected by her and by their classmates. Sometimes this message was communicated implicitly, such as when at the start of the school day students greeted one another by name while looking each other in the eye and exchanging high-fives (observation, 9/29/14). Often, this message was also communicated explicitly. For example, in one math lesson, Karen explained instructions for a learning activity with partners. In this activity, students would see the answer their partner recorded. Karen asked students to brainstorm ideas for how they might respectfully tell their partner that they had written down the wrong number. Students made suggestions including, "Excuse me, could you please look at the card to make sure you got it right?" and "You could say nicely, 'Your number is upside down'” (observation, 11/17/14).

Students also volunteered ideas for interacting with their peers in supportive ways without Karen's prompting. During the same math lesson, as Karen was giving instructions for a different learning activity involving a game, a student announced to his classmates that they shouldn't make fun of their partner if they rolled a higher number on a set of dice and got to go first in the game, and later, that they shouldn't make fun of their partner if they ended up winning the game. Although Karen had not solicited these comments, she delayed giving instructions when the student contributed them to ask the 
class how it would make their friends feel if they got made fun of because they did not win. Students responded that it would make their friends feel bad (observation, 11/17/14).

Karen also worked to develop a community that was accepting of differences in which students recognized that their own needs might be different from their peers', and their teacher would respond accordingly. She explained:

We also talk about how I have different expectations for different students, so one student...in particular, he has difficulty focusing sometimes, so sometimes I'll even sit him on my lap or let him put his head up here [gestures to knee], and so I got asked, "Why is so and so allowed to do that?" And I laughed, and I said, "That's a good question! Because some people can focus from back there, and some people need to be right beside me." So talking about the expectations. (interview, 11/17/14)

Karen felt her other students understood that this student had a need that they did not, and that as a result, he would receive a different kind of support to stay focused than the rest of the class. This aspect of the community in Karen's thoughtfully created learning environment reflected a philosophy of classroom leadership grounded in responding effectively to student differences. When Karen communicated to her students that different learners come with different needs, and that in her classroom, those differences would be recognized and responded to, she invited them to share in her vision of a learning environment in which everyone would receive what they needed to grow.

Student mindset. Karen's growth mindset toward her students' development served as the foundation for her beliefs about learning environment and leading the classroom. She summed up this attitude by explaining that the expectation in her classroom was:

Always do your best. That if you're doing your best, no one would ever be disappointed, and you should not be disappointed in yourself. And everyone's 
best looks different...[B] ut just doing the best you can personally do, and what you know how to do....My assistant and I really try to focus on the effort you put into things. Sometimes we'll say, "Be brave and take a guess." (interview, $11 / 17 / 14)$.

Karen felt that her students generally were "brave" as they tried new things and pushed to do their best. She noted that parents often asked her whether their children would get frustrated, not want to try something, or "shut down" when something was hard in school, and Karen always responded that, in her class, they generally did not (interview, 11/17/14). She attributed this to the classroom learning environment, in which it was expected that students would sometimes make mistakes as they did hard work.

During one math lesson, Karen reminded the students of this belief (observation, 11/17/14). She asked students whether it was all right for their friends to make mistakes, and the class responded that it was. She continued, "Yes, it's okay to make mistakes. You make mistakes. I make mistakes. We all make mistakes. That's why we practiceto get better. Are we all practicing different things as we grow?" (observation, 11/17/14). The students responded yes. Karen paired this attitude that it was normal to make mistakes as one practiced to get better with an attitude that facing academic challenge was an exciting thing. For example, during small group work in math class when Karen presented a harder addition problem, she said in an enthusiastic tone of voice and with a broad smile, "Now it's time for a challenge! We're going to do two more than the number!" (observation, 12/08/14). Almost every student in the group of eight smiled, laughed, squealed, or clapped their hands after Karen said this. Karen expressed this excitement every time she presented students with an especially challenging problem during the lesson, and the students responded with excitement each time (observation, 12/08/14). 
Karen's growth-minded attitude toward her students influenced the way she addressed off-task behavior. Rather than responding with anger and punishment, Karen instead reminded students of her expectations. In one instance typical of her response, Karen noticed a student working on an independent activity during language arts rotations was staring off into space. Karen made eye contact with the girl and said, "I need to see you writing." The girl responded by frowning and then pouting. Karen responded in a calm but firm voice, "I expect more from you." The student then returned to writing (observation, 11/17/14). By November, Karen also encouraged students to evaluate their own behavior to determine whether it was on-task and consider the ways in which they might improve their performance. Each time students rotated through a series of independent and small group activities, Karen asked them to reflect on several questions she posed and give themselves a score. On one occasion typical of this type of reflection, Karen said to her students:

I want you to tell me how you did during the language arts lesson....I want you to think about this and ask yourself: Did you say the poem out loud? Did you write in your journal independently? Did I have to remind you that I needed to see you reading and writing? I want you to show me a one, two, or three. A one is: Uh, I could have been more focused, I didn't work the whole time, and Ms. Karen had to ask if I was working. Give yourself a two if you did pretty well, but you could still do better. Give yourself a three if you did the best you could do. Close your eyes. Now put your fingers up in the air. (observation, 11/17/14)

After several students raised only two fingers and the class then put their hands down, Karen said, "Thank you to the people who are being honest with their feedback and their own reflection. It's hard to admit it when we could have done a little better" (observation, 11/17/14). By doing this, Karen helped students cultivate an awareness of their own performance and recognize ways in which they might improve.

By the end of the fall, Karen appeared to have succeeded in establishing a 
classroom learning environment, through the arrangement of the physical space and creation of a positive climate, in which students felt comfortable and safe enough to take risks, make mistakes, and target their own improvement in order to grow.

Classroom management. Karen's classroom management was closely related to the learning environment she established. It reflected the belief that, despite their young ages, her students were capable of following instructions, carrying out classroom procedures, and working independently. Throughout the fall, Karen managed the running of her classroom in a way that prepared students to work on individual or small group assignments successfully without adult involvement.

Classroom procedures. Along with arranging the physical learning environment and positive classroom climate, management issues were Karen's primary focus for the first four to six weeks of school. She described the core of this focus as "setting up routines and procedures clearly for them, and really modeling that for them, and having them model the correct and incorrect way, and holding them accountable to that once they've learned it" (interview, 9/29/14).

When I visited her classroom for the first time during the fourth week of school to observe a language arts lesson, I watched Karen's students perform routines and procedures for independent reading and independent writing without any re-direction, including knowing how to retrieve materials, where to go in the classroom, what to do and not to do while working, and how to transition back to a whole group meeting at the conclusion (observation, 9/29/14). By my second observation in October, students also executed routines for using a chart to determine their small group's next learning center during a lesson, responding with patterns of sounds to the teacher's vocal cues for getting 
their attention while stopping their work and looking at her, and cleaning up materials at the end of a learning activity (observation, 10/16/14). During this observation, only two students needed prompting beyond one cuing announcement for the routine to use the chart to find their next learning center; all of the students completed the other routines with no prompting or correction (observation, 10/16/14). In my third observation in November, in addition to executing the routines I had seen previously, all students transitioned between learning centers without correction or prompting when cued only by the sound of a rain stick (observation, 11/17/14).

Karen attributed the apparent ease with which students followed established routines in part to the care with which she had taught the routines, had students practice them, and held students accountable for following them, often by asking students to repeat a routine several times if they did not execute it correctly the first time (interview, observation, 9/29/14). She also frequently reminded students of the expectations for the successful completion of a procedure. In a typical example, she had students call out a chant of the expectations for independent reading, part of the Daily 5 (Boushey, 2006, 2014) literacy framework, before they began the activity: "Read the whole time, stay in one spot, quiet voices, building stamina, and get started right away" (observation, 9/29/14). She also designed procedures to avoid anticipated problems. In another typical example, I observed Karen give a small group of students instructions about getting materials for an activity. I recorded in my field notes:

She points to the stack of materials in containers on her left and says that, when she calls their names, she is going to give them a paper, and then they can get a clipboard and a pencil from the containers next to her. She then asks, "Who can tell me what three things you're going to need today?" One student says a paper. Another student says a clipboard and a pencil. Karen asks if it matters what color clipboard you get. One student calls out loudly, No! Karen says that the color of 
the clipboard doesn't matter and that the group is just going to take one quickly and go. (observation, 12/08/14)

This exchange suggests Karen anticipated that student concerns over the color of their clipboards would cause them to get their materials more slowly. She therefore prefaced the procedure of getting materials by reminding students to take one quickly and sit down.

Last, Karen devoted significant time to ensuring students understood classroom procedures and task instructions completely before asking them to engage in independent or small group work. She would often spend ten to fifteen minutes reviewing the process for completing self-directed learning activities before students would begin engaging in them, particularly in math instruction (interview, 11/17/14). During one classroom visit in November, I observed Karen spend ten minutes explaining the procedures for the "Roll a Turkey" game that would serve as an independent activity at a learning center for math rotations that week. During this period, Karen (a) showed students the materials for the game; (b) reviewed the instructions page that diagrammed how to play the game; (c) modeled playing the game with another student, simulating multiple rounds of play while students reminded her what she should do next; and (d) asked students to brainstorm solutions to problems she anticipated, such as deciding who would go first and what to do if they could not remember how to draw a particular turkey body part (observation, 11/17/14). Karen attributed her students' ability to complete independent and small group activities during learning center rotations without asking her questions to the fact that she carefully reviewed procedures beforehand (interview, 11/17/14). During the math lesson I observed in which students worked independently or in small groups without Karen's or Susan's involvement, no student asked any questions about classroom 
procedures or needed reminding about them (observation, 10/16/14).

While Karen's management of general classroom procedures enacted by students proceeded smoothly during the fall, a management issue involved in instructional planning presented significant obstacles.

Structuring rotations. The single most challenging issue Karen faced during the fall of her first year in the classroom involved the "logistics" of structuring learning center rotations based on readiness grouping (interview, 9/29/14). While Karen often couched this as a challenge in designing classroom procedures (interviews, 9/29/14, 10/16/14), she also recognized that the issue involved complex relationships among curriculum, assessment, and instruction in her classroom (interviews, 10/16/14, 11/17/14, 12/08/14). Because Karen's learning center rotations and grouping practices reflected her core efforts to modify her instruction in response to student differences, I discuss them below in the Modification of Instruction section.

Curriculum. In the spring, Karen had characterized high quality curriculum as critically important to effective differentiation, commenting: "I think that the key is having the high quality curriculum that- to differentiate. Because in the end you can differentiate and differentiate flat curriculum, and it's still gonna be flat. I feel like that's-it's just the foundation" (interview, 2/26/14). Karen viewed high quality curriculum as grounded in essential understandings, relevant, and connected to students' personal experiences (interviews, 1/27/14, 2/26/14). Before the school year began, Karen hoped to build an interdisciplinary curriculum around the key concept of perspective and to identify core essential understandings reflected in standards to function as her curricular framework (interview, 9/01/14). During the fall, however, Karen's focus was 
on simply keeping pace with creating brand new curriculum in every subject to teach each week, rather than on ensuring the curriculum she created demonstrated the hallmarks of high quality.

Curricular resources. As a private school, Newland Academy did not follow mandated state standards. Instead, the model Common Core Standards guided its curriculum design. As the only kindergarten teacher in a school without an instructional coach, Karen developed the curriculum for her language arts, math, science, and social studies classes by herself. No aspect of her curriculum was prescribed beyond an expectation that it would address the first grade Common Core Standards for English Language Arts and Mathematics. Karen was asked to cover first grade standards since the preschool program at the school addressed the kindergarten standards, although only four of Karen's 17 students had attended Newland for preschool (interview, 9/29/14). Karen did not inherit any materials from her predecessor and therefore created all her own materials from scratch. She construed creating totally new curriculum by herself as one of the greatest challenges she faced in her first year (interview, 9/29/14). Karen explained:

There's no prescribed curriculum, which is great in some ways, but also what's given me the most difficulty so far as a new teacher. And I think as a fifth year teacher, I'd be like, all right, I'm raring to go. I know exactly what to do. But that's really difficult for me - to have no set thing. I mean we have standards, but beyond that, we don't have set-out curriculum. So I think that makes it tricky too. It's more time-consuming too to come up with these things. And then to have, you know, right now I have ten math books sitting at home right now... and so it's like, well, decide your topic and then see how they [the books] teach it and then see how you want to teach it, and for me, I'm like, Oh my gosh! It's just overwhelming. (interview, 11/17/14)

Newland Academy did not have an adopted textbook series for any of its subjects. The school did provide Karen with teacher's editions from several math series and owned a 
number of manipulatives. For language arts, the school owned guided readings books, rather than basal readers, and used Daily 5 (Boushey, 2006, 2014) and literacy work stations (Diller, 2003) as frameworks for literacy instruction. These frameworks, on which Karen received training during summer 2014 before she began teaching (interview, 9/01/14), offered suggestions for instructional and classroom management structures to support elementary students' independent and small group work in literacy. Karen had access to a few Full Option Science System kits but had no other materials to support science or social studies instruction.

Pacing guides. Karen did not develop a pacing guide or other formal curricular document for language arts during the fall. Instead, she said she relied on her "general knowledge of reading instruction from grad school— just knowing where the kids need to start, that the kids need a firm concept of word before moving into books, that kind of thing" (interview, 10/16/14). Karen did develop a formal math pacing guide in September for the first quarter outlining the topics she planned to cover and the Common Core Standards she would address (interview, 9/29/14).

Karen began this process by searching on the Internet for $1^{\text {st }}$ grade pacing guides based on the Common Core and locating two that she liked. She used these two resources to create her curricular framework, in which she outlined two readiness levels for many skills she included to support differentiation (interview, 9/29/14). Karen described:

I had the two examples to look at. They did things a little bit differently. So like one of the pacing guides only had the kids doing addition within 10 the first nine weeks. The other pacing guide had them doing addition and subtraction within 10. So I was kind of thinking like, all right, I'm going to put both of them down there, so if I see that my lowest readiness group is not quite ready for addition and subtraction, just doing addition. So just kind of seeing for myself, this is one way 
they've set it up. This is another way you could set it up. So that when I'm going through, I can quickly see different options for how to differentiate. (interview, 9/29/14)

While Karen's pacing guide for the first quarter provided her with clarity surrounding higher and lower readiness levels for specific skills, she subsequently came to feel that she lacked this clarity about the content due to the wide range of readiness variance in her class. In light of this, Karen felt uncertain about the progression of skills her curriculum should address at different readiness levels and how to teach them: "I have a hard time knowing what comes next, or how do I teach something, like for my lowest-number recognition. Is it just simply drill and kill—like this is 31 , show me 31 , make 31 in tallies, or that kind of thing?" (interview, 11/17/14).

As detailed in greater depth in the New Rotations Structure section below, Karen ultimately contacted an instructor from graduate school for guidance on this issue in midNovember. Her former instructor gave her the kindergarten and first grade teacher's edition of a textbook series she did not have, along with some other materials, so that she could reference the progression of skills outlined in its detailed scope and sequence. Karen described, "I had a hard time knowing what to teach first and when, and so the program overview gives the whole overview of each topic and then breaks it down for each individual lesson, so that's really helpful for me to see too" (interview, 12/08/14). She explained that she was now teaching lessons as they were outlined in the book and using worksheets from the materials she received from the instructor, which she had not used previously in her instruction. Karen noted that she did sometimes make a more challenging version of a worksheet for higher readiness students, concluding that she used the new materials “as a guide, but I don't feel glued to it” (interview, 12/08/14). 
The freedom Karen had over her curriculum both supported and detracted from her implementation of differentiation. While it left her "free to make decisions [that] are best for the kids" (interview, 12/08/14) and gave her flexibility with pacing (interview, 11/17/14), developing her own curriculum without many prescribed structures or materials provided consistent challenge: "Challenge because there is so much freedom, it's kind of like you don't have anything to go off of. If I had something to go off of, I could be like, Oh, I can tweak it here, tweak it there, but starting from ground zero is difficult, so I'd say that's how it challenges implementation" (interview, 12/08/14).

Assessment. Karen's use of assessment during the fall was focused on student readiness in language arts and math. While Karen regularly gathered informal formative assessment data from interactions with students during individual conversations or small group instruction (interview, 9/29/14), she also made decisions about grouping students and modifying instruction based on formal pre-assessments completed at the beginning of the year in language arts and math. In early September, Karen gave students an individual oral math preassessment she designed that asked them to recognize written numbers from one to 20 , count as high as they could up to 100 , recognize shapes, and recognize colors (interview, 9/29/14). In October, Karen administered the Phonological Awareness Literacy Screening (PALS) to all students (interview, 10/16/14). For Karen, the variance in student readiness indicated by these assessments underscored the need for modified instruction in her classroom (interviews, 9/29/14, 10/16/14). Karen identified four general readiness levels for language arts instruction and four general readiness levels for math instruction based on patterns in this pre-assessment data and grouped students for instruction accordingly. In math, these groupings did not change during the 
fall, as Karen believed based on her informal formative assessments that their readiness

levels remained the same with varied skills students practiced based on their depth of number concept. In language arts, these groupings did not change in October and November, although Karen planned to begin using PALS “quick checks" in December to formally reassess reading readiness (interview, 11/17/14).

Modification of instruction. While Karen often incorporated student choice based on interest into her instruction, such as allowing students to select their own books for independent reading (interview, 9/29/14; observation, 9/29/14), responsive instruction based on readiness was her primary focus for differentiation during the fall (interview, 10/16/14). Addressing the "wide range" (interview, 9/29/14) of student readiness revealed by math and language arts assessment data proved to be challenging for Karen. In math, some of Karen's students had number concept that was "what you'd expect of end of first grade students," while others had only "very early development of number sense" (interview, 11/17/14). This variance in readiness also stood out to me in observations. During one classroom visit, I recorded the following in my field notes:

A student at the table on my left where the group is playing Memory says, "These four cards make 40 salamis!" I look over and see that he has a stack of four cards in front of him, which all appear to have the same images on them. The first card shows the numeral 10, along with pictures of 10 sausages on it. [Analytic Note: The student has either added $10+10+10+10$ or multiplied 10 X 4 . I am reminded of how Karen told me during our first interview that one of her students was already multiplying at the beginning of kindergarten. I am struck by the variance in readiness in number sense between this student and the students Karen is working with in her lower readiness group who are working to understand what the number 10 represents.] (observation, 10/16/14)

Similarly, PALS data indicated significant variance in readiness, with Karen's class including one student reading on a third grade level, another reading on a second grade 
level, and many who did not yet recognize all the letters in the alphabet (interview, $10 / 16 / 14)$.

Responding to readiness variance. Karen modified her instruction to respond to this variance in student readiness in two ways: through her one-on-one interactions with students and the design of her learning rotations. Her focus was on undertaking these approaches in her language arts and math instruction. "I can't focus on everything as a first year teacher” (interview, 10/16/14), Karen explained during an interview, recognizing the unique demands of this phase of the ongoing process of learning to teach. She continued, "I think for reading and math those two are more, it's like more apparent to see the differences in readiness levels than anything else" interview, 10/16/14). While she hoped to implement some interest-based differentiation in science and social studies instruction at some point during the year, she concluded, "I'm most concerned right now about getting started with the readiness-based differentiation" (interview, 10/16/14).

Individual interactions. Karen referred to one aspect of modifying her instruction based on readiness as "differentiating through the support I give and the kinds of questions I ask" (interview, 12/08/14). An example of this occurred when I observed Karen interact with students during independent writing time (observation, 9/29/14). Since I could not hear everything Karen whispered to each student, she later explained to me the nature of their conversations during an interview:

But like you saw me go over to a few kids, because one gets really anxious during writing time if he feels he doesn't know what to write about, so I always go over to him because I just want to ease his anxiety and get him writing. And then with the little boy up here, he has a hard time doing it independently, listening for sounds, like, all right, what do you want to write about? And then what's your first word? And then that kind of thing. So the ones I have seen can do it independently, I just let them go. So writing is a big difference, like some of them are listening for sounds and understanding that letter-sound connection. 
Some of them, a few are probably just writing random letters. If they're not yet ready to write and sound out letters, then drawings and adding details to drawings and how your picture can tell a story. So, a lot of right now is just coming from my observations of what I see they can do during our small group time or when we have a small writing assignment, it's easy to see how is sounding out words, who is more comfortable, and who maybe is not, who requires more support or prompting. (interview, 9/29/14)

With these comments, Karen identified the nature of different writing readiness levels among her students, specific needs different students presented when they worked on the assignment, and examples of how she responded to those needs by "just letting them go" or providing "more support or prompting." This example was typical of Karen's attunement to individual student readiness levels and targeted responses to them.

Learning centers. Learning center rotations served as the instructional framework for language arts and math in Karen's classroom. As discussed above in the Assessment section, patterns in assessment data indicated four readiness levels for language arts instruction and four readiness levels for math instruction to Karen. In October, after students had learned routines for transitioning among centers and had practiced reading and writing independently through the Daily 5 literacy instruction framework, Karen had students rotate through four learning centers in language arts and math class.

Karen noted that having students work independently or in small groups at the kindergarten level could prove "tricky" compared to doing so with older students. She explained:

It is different... when the kids are not yet reading and you're teaching them how to be independent. I'm learning that now that I'm in it that it is more difficult because I can't just hand them a set of directions for a math game and say, go do this independently. And that is what I think is tricky-balancing getting them to practice doing it first and tons of modeling, and then being able to build their independence, whereas maybe a higher grade level they already have some independence built in. (interview, 9/29/14) 
Karen's explanation demonstrates how the success of her learning centers as a response to variance in student readiness was closely related to her students' proficiency with classroom procedures. She characterized the fact that her students could not read as a challenge to her implementation of differentiation (interview, 12/08/14). However, because Karen spent the first four to six weeks of school teaching students how to work independently in the classroom and she spent considerable time explaining and modeling the instructions for her non-reading students, her students could complete learning centers successfully in her room.

Every day in language arts class, students rotated through four of the Daily 5 centers: guided reading with Karen, Word Study to practice spelling with Susan, independent reading called "Read to Self" time, and independent writing called "Freewriting" (interviews, 9/29/14, 11/17/14; observation, 11/17/14). Guided reading, Word Study, and Read to Self were differentiated by readiness, while all students had the same assignment for Freewriting. In guided reading, Karen had students in different groups read different books or poems based on their reading level. In Word Study, Susan provided students in different groups with different words based on readiness with spelling. During Read to Self time, Karen sometimes assigned students books to read based on readiness level and sometimes allowed students to choose based on their own interests (interviews, 9/29/14, 11/17/14).

While the Daily 5 instructional framework used by all elementary teachers at Newland Academy provided Karen with a well-defined structure in which to differentiate literacy instruction, Karen struggled throughout the fall to determine how to structure her 
math instruction. At the end of September, Karen had not yet instituted learning center

rotations in math, as she remained uncertain as to how to approach it. She explained:

[W]hen they're doing independent work in language arts, it will be writing and reading to self, and that is just easier to differentiate because... when they're doing reading independently, they're all reading something. Now what they're reading is changing, and that's how I'm differentiating that, but with math, it's more difficult, because....They're not going to be doing the same kind of activity necessarily....the lowest readiness group will be doing something most likely pretty different from the highest readiness group. (interview, 9/29/14)

For Karen, the modification of literacy instruction seemed more straightforward and did not require her to give different sets of instructions for different tasks to different groups of students. Whether her students were still working to identify letters of the alphabet or were ready to read chapter books, all would do independent reading. Their reading selections would just be differentiated.

When Karen considered her math instruction, however, she believed that her lower readiness students would often need to work on different activities, rather than different versions of the same activity, and she was unsure how to structure that, especially since she needed to explain all activity instructions orally. Karen described the confusion this created for her:

I'm still figuring out... how to structure my math time. Are we doing rotations?... How am I going to explain to one group that they're doing something different than another group during their independent practice time?... That's what I also kind of mean by having so much freedom [with curriculum and instruction at Newland], because it's not dictated-you teach math this way...I've got the steps to start differentiating, but logistically, it's difficult to think about how that's going to work....How would I meet with each group to introduce new things when the groups will be learning new things?...If one group is going to be playing a game, are they playing the same kind of game but with different numbers? (interview, 9/29/14)

By October, Karen had sorted through some of this confusion to design a learning center rotation structure for math. 
Structuring rotations. In this new structure, students rotated through four learning centers every day in math readiness groups based on preassessment data. Each rotation lasted around 15 minutes or less. In two of the centers led by Karen or Susan, students completed an activity that was differentiated by readiness. The other two centers involved activities or games that were not differentiated that students completed individually or in small groups (interviews 10/16/14, 11/17/14; observations, 10/16/14, 11/17/14). For example, during one observation, students (a) worked with Karen using a number line and other manipulatives on concepts involving number sense and skip counting, (b) worked with Susan on concepts of less and more using manipulatives, (c) played a memory card game in small groups, and (d) completed an individual activity in which they recognized numbers in a pattern set and practiced writing numbers. When working with Karen or Susan, students with higher readiness examined concepts in greater depth through more challenging problems (interview, 10/16/14; observation, 10/16/14).

Students generally worked through all or most of the rotations every day on Tuesdays through Fridays. On Mondays, Karen spent the entire math lesson explaining the four individual or small group activities that students would complete that week independently during rotations, doing two of the activities on Tuesday and Wednesday and two of the activities on Thursday and Friday. During these Monday instruction periods, Karen spent 10 to 15 minutes explaining and modeling how to complete each activity so that students would have great clarity surrounding the instructions and expectations for successful completion (interview, 10/16/14, 11/17/14; observation, 11/17/14). Karen explained, "I found it easier to do that because then I can take all the 
time to explain, and that's what's hard is when you're introducing a game for them to play independently, you need to be very explicit, so that I'm not interrupted with my group and they know how to play the game independently" (interview, 1016/14).

New rotations structure. Karen was not fully satisfied with this instructional framework for math. She felt that 15 minute rotations were not long enough to engage students in conceptually deep thinking and that they felt "rushed" (interview, 11/17/14). She also felt that her highest readiness groups often would not benefit from the same independent activities as her lower readiness groups but could not determine how or when to give them instructions for a different activity (interviews, 10/16/14, 11/17/14).

The independent practice is what I'm having the hardest time with. It's first of all when do I explain to these groups that they're doing something different without the other groups picking up on it? And how do I explain it without telling the other groups because the other groups will get confused? Like logistically finding that time, and then making it so I know what they can do independently also....Like today what I'm going to introduce is everybody's going to be doing the same thing for independent practice, and I don't like that, but it's the best I can do right now until I can do better. (interview, 11/17/14)

Karen's dissatisfaction with the structure of her learning center rotations ultimately led her to ask a former math methods instructor from graduate school for help.

The instructor encouraged Karen to combine the two highest readiness groups and the two lowest readiness groups for math instruction so that there would be a total of two groups instead of four, and to have Karen teach one group for the entire math lesson and Susan teach one group for the entire math lesson. Karen adopted this new structure in late November. She taught half of the class for two days while Susan had the other half, and then Karen and Susan switched groups for another two days (interview, 12/08/14; observation, 12/08/14). This eliminated the independent activities from math time and meant that all of the students in each group received the same instruction. 
Effects of new structure. Karen believed that the new structure was an improvement over the original structure (interview, 12/08/14). Students no longer rotated among centers and therefore worked on the same activity for a much longer period of time, which Karen felt allowed for deeper exploration of concepts. With the first structure, she had felt like her activities were "scattered and all over the place" (interview, 12/08/14), while the second structure allowed her to spend more time and thought on planning one lesson targeting one concept and making only two versions for higher and lower readiness.

Karen noted that, now that four groups had been combined into two, there was wider readiness variance within the groups. She reported differentiating her instruction when working with each half of the class, in which all students in the group "get the same kinds of problems and the same worksheet" (interview, 12/08/14), by providing:

more or less support [to students within the same group], so some of them I'll say [aloud] a word problem, some of them need a lot more prompting, some of them need to use manipulatives, some of them need more guidance, and so that's how I differentiate within the groups right now....[W]hen we're moving through the lesson, there's a guided practice portion and then what's supposed to be independent practice, and so if I feel that students are ready to work independently, I kind of let them go. Yeah, it's mainly differentiating through the support I give and the kinds of questions I ask them. (interview, 12/08/14)

I conducted one observation of a math lesson with this new structure in place, however, and I did not observe any examples of varying levels of support during instruction through teacher student interactions (observation, 12/08/14).

During this observation, I watched Karen work with a group of eight students who completed a series of worksheets from the materials she received from her former university math methods instructor (observation, 12/08/14). Karen read the instructions out loud, and the students filled in the blanks on each page. Some students completed the 
pages much more quickly than others, and Karen asked them to wait until their peers

were finished to move on. At one point, the three students who correctly completed a page first out of the group waited for over ten minutes for the rest of their classmates to complete the page. I recorded in my field notes:

Karen is speaking quickly to the students who are not finished and keeps telling them to correct things that are wrong. She is making quick motions to point at their papers as she does this. [Analytic Note: She seems to be rushing them through their work, aware of the fact that the faster students are waiting.] At 11:30, seven students are now done, but one...is not done and is not writing. [This student has not written the answer, 9, correctly.] Karen puts the card with the 9 in front of her. She says, "Go quickly, we're waiting on you." The girl erases what she has but does not write anything. Karen asks, Does your 9 look like this 9 ? The girl traces the 9 on the card with her finger, but she still doesn't write it. Karen says, Which side is the line on? The girl points to the right. Karen says, Yes. The girl still doesn't write anything. Karen says in a slightly frustrated voice, Write your 9 now. The girl writes a 9 correctly. Karen says, "You need to stay focused." She tells the girl to turn to the fourth page, which all of the other students are on. (observation, 12/08/14)

Before Karen moved to the new structure for math instruction, the students who finished the page quickly had been in a higher readiness group than the students who took most of the ten minutes to complete the page. In this instance, Karen's comments seemed designed to hurry the last student along, rather than to provide support in light of differences in readiness.

As of December, Karen planned to continue to base her math instruction on worksheets she received from her former math methods instructor and to teach to two readiness groups, with Susan, who is not a licensed teacher, delivering half of the instruction. Karen concluded:

I understand that it's not perfect, but for right now, it's what I have to go off of, and it's the best I can do now. And so at least it gives me something to go off of. Now if the lessons aren't perfect or they're not the most challenging or the most real-life scenarios, I understand, but it's better than what I was doing.... But then there's always a part of you that wants to keep growing and keep making it 
better...And as I get more into it and as we do it, from now until spring break...I think spending more time in it will also help me see what areas I might need to improve as well. (interview, 12/08/14).

Mindset. Although Karen was not fully satisfied with her implementation of differentiation's principles and practices, this did not discourage her. On the contrary, Karen said that she would always hold high professional expectations for herself, which she defined as "growing as an educator, and not just being content with where you are. Always trying to learn more" (interview, 9/01/14). As a preservice teacher, Karen had anticipated that she would make mistakes and hoped that she would be patient with herself when she did so (reflection, 2/02/14). As a first year teacher, Karen was working to achieve this. She described:

Well, I've experienced a huge learning curve that I knew would happen during my first year of teaching, and I think it's hard to find that balance of like being content with doing the best you possibly can do at the time. So it's hard to be comfortable doing the best you can and not beating yourself up about it while still pushing yourself forward, to find the balance, I think. At the end of the day, saying, "Phew! I know I did the best I could," but also still saying, "OK, these are the ways I can get better," without being overwhelmed by it too, if that makes sense. I'm constantly thinking of ways to improve what I do. I'm constantly reflecting on what I do. Like at the end of each day, the end of each lesson, the end of each group [rotation during a lesson], I'm like, "OK, that didn't go so well," or "I could have been a little more patient here," or whatever...I am finding that balance of, keep improving but do the best you can. (interview, $11 / 17 / 14)$

Karen viewed this search for balance between contentment with her own best efforts and reflection on ways to improve her teaching as a manifestation of her growth mindset, which she saw as supporting her implementation of differentiation. When she compared her mindset as a preservice teacher to her mindset as a first year teacher, she felt that some aspects of her job supported a growth mindset and others supported a fixed mindset: "Now that I am a teacher...I can see how I'm learning to do things better, but I 
can also see my areas of weakness more easily because it's me doing it fulltime five days a week all day" (interview, 11/17/14). In this way, Karen recognized conflicting influences on her mindset — in her practice, she could see both her own mistakes and her own improvement with clarity. Overall, however, she felt that her mindset had shifted slightly toward more of a growth mindset than she had had as a preservice teacher, especially since by November she could see significant growth in her teaching practice compared to the beginning of the school year (interview, 11/17/14). She also saw her willingness to reach out to many resources for help as a manifestation of her growth mindset, since she saw it as a demonstration of her strong commitment to improve her practice (interview, 11/17/14, 12/08/14).

2(b) Summary. Karen implemented many practices reflecting key principles related to all of the non-negotiable elements of a differentiated classroom. During the first month of school, Karen's focus was on establishing a supportive learning environment and efficient classroom management procedures. Her focus then shifted to issues involving curriculum, instruction, and assessment, particularly those that involved the modification of instruction by student readiness.

Karen established a classroom learning environment, through the arrangement of the physical space and creation of a positive climate, in which students felt comfortable and safe enough to take risks, make mistakes, and target their own improvement in order to grow. Her classroom management reflected the accurate belief that her kindergarten students were capable of following instructions, carrying out classroom procedures, and working on individual or small group assignments successfully without adult involvement. The freedom Karen had over her curriculum design in all subjects both 
supported and detracted from her implementation of differentiation, since it offered her flexibility with decisions about content and pacing but also meant she designed curriculum from scratch with limited resources.

Karen's use of assessment during the fall was focused on student readiness in language arts and math. While Karen regularly gathered informal formative assessment data from interactions with students, she also made decisions about grouping students and modifying instruction based on formal pre-assessments completed at the beginning of the year in language arts and math. Karen's primary focus for differentiation in the fall involved modification of instruction in response to a wide range of readiness revealed by math and language arts assessment data. This presented Karen with challenges, especially as she struggled to determine the best instructional framework for math. While Karen was not fully satisfied with either approach to math learning rotations she used in fall 2014, she was optimistic about continuing to improve their structure in spring 2015. Her growth mindset was reflected in her balancing satisfaction with the knowledge that she had tried her best in her instruction against constant reflection on ways to strengthen her teaching practice.

\section{Research Question 2(c): How did contextual factors relate to Karen's conceptions of the model and her teaching practice?}

When Karen began teaching at Newland, she encountered a host of factors involving the school's culture of practice, the nature of her position as its only kindergarten teacher, and who Newland's kindergarten students were that shaped the ways in which her conceptions of differentiation were and were not reflected in her teaching practice during the fall of her first year. While a number of these factors 
challenged her implementation of differentiation, many others supported her efforts to align her teaching practice with her philosophy of responsive instruction. Together, these factors created the complex context Karen navigated to work toward this alignment.

Culture of practice. "I'm excited but also a little nervous," Karen commented during an interview soon after being hired at Newland Academy. "They do real teaching at this school" (4/10/14). By the conclusion of the Newland interview process, Karen's impression of the school was that the community expected its teachers to teach with student engagement and rigor as goals. Throughout the fall, Karen continued to share her impressions of Newland's philosophy and the ways in which it was put into practice by its administration and teachers. I also interviewed Beth, the school's founder, current principal, and only administrator, to learn her beliefs about Newland's culture. During my separate interviews with them, when Karen and Beth discussed the philosophy of Newland Academy, their messages about its content and application in the daily running of the school were almost indistinguishable.

School philosophy. Karen wanted to teach at Newland because she believed the philosophy of the school, as it was described to her during the Newland interview process, aligned with her own philosophy of teaching and learning. Karen explained what had appealed to her in her initial conversations with Beth:

I just remember when I was going through the interview process Beth saying, we hold high expectations for our teachers, and we hire the best teachers. We hire people who keep pursuing excellence and who want to grow as educators, so I remember that specifically sticking out because that's one of the reasons I wanted to come here, because I felt personally that's the kind of person I am, so to know that they have that growth mindset and that they do want the best from their teachers. (Karen, interview, 9/01/14) 
As the fall progressed, Karen came to recognize that the school's philosophy was in practice the same as it had been described to her in the abstract as a job candidate.

Newland's general philosophy. Karen and Beth both described Newland Academy as having a culture of excellence in which the administration has high expectations of teachers and teachers have high expectations of themselves (Karen, interview, 9/01/14; Beth, interview, 12/11/14). The foundation of these expectations was a growth mindset, which Karen described as "always trying to improve and to be better than you were the day before" (Karen, interview, 9/01/14). Teachers at Newland were also expected to develop "meaningful and purposeful curriculum" (Karen, interview, 9/01/14) that reflected both joy of learning and rigor (Beth, interview, 12/11/14). Constant collaborative teamwork and community building among all school employees created what Karen called a "united front on all accounts" (Karen, interview, 9/01/14; Beth interview, 12/11/14). The shared understanding that united Newland's teachers was that they would always do what was best for the students (Karen, interview, 9/01/14), which required "knowing your students and getting your head out of the textbook and realizing that the mystery of what you're trying to teach isn't the subject — but the children in the room" (Beth, interview, 12/11/14). A "loving, nurturing environment, where we have zero tolerance for anything except kindness" existed alongside a physical environment designed to feel comfortable and "homey" for students (Beth, interview, $12 / 11 / 14)$, the goal of which was to make the school a place where students looked forward to coming every day (Karen, interview, 9/01/14). At Newland, teachers were encouraged to try new strategies and approaches in their classrooms to meet the needs of students (Karen, interview, 12/08/14), with the administration communicating to teachers 
that "You have the freedom to teach the way you believe. We hired you because you're a professional. We want you to take risks. We know sometimes it's gonna fail. If you're not making mistakes, you're not trying hard enough" (Beth, interview, 12/11/14). This explicit permission for all teachers to try new approaches and make mistakes enhanced Karen's willingness to differentiate instruction.

Newland's philosophy of differentiation. At the core of Newland's philosophy and expectations for teachers was Tomlinson's model of differentiated instruction. Beth had studied the model extensively and attended workshops with Tomlinson. Part of her decision to found the school was to put the model into practice authentically, in a way other schools in which she had worked had not. She often hired new teachers who were prepared to differentiate instruction and sent other faculty to workshops to learn about Tomlinson's model in depth (Beth, interview, 12/11/14).

Beth defined differentiation, as it was reflected in Newland's classrooms, to mean:

Knowing each child as an individual. Who they are. What inspires them. What frustrates them. What avenue I can take to reach them. Just connecting with each of them.... It's also teaching the growth mindset as a daily ritual of curriculum from preschool through middle school.... think that's the most important thing that we can teach them to do, whether it's physically, emotionally, if our teachers aren't growing, if I am not growing, the kids aren't growing, we're not doing our job.... I know each of them as an individual. They're all different as an individual. It's just a matter of being respectful to each individual child. And I think that word is the essence of what it is. It's a respectful environment. We expect them to respect us, and we teach them respectfully, so if they already know stuff and need more challenges or if they're struggling or if they're not interested, we find avenues to reach them no matter what out of just being respectful to them. (Beth, interview, 12/11/14)

This definition of differentiated instruction emphasized knowing students as individuals, viewed supporting the growth of those individuals as the purpose of teaching, and 
expected teaching students respectfully in response. Respectful teaching in this context meant modifying instruction based on individual need to "reach them no matter what." Newland Academy had many multi-age classrooms including students from two grade levels. Beth explained that the school used this structure because it required teachers to differentiate: “I think you can't survive in a multiage classroom unless you differentiate. But also it helps to kind of blur so it's not like, this is my high group, this is my weak group" (Beth, interview, 12/11/14).

Karen described differentiation as a topic that teachers at the school discussed constantly (Karen, interview, 11/17/14). She noted that implementing this instructional approach effectively was a "common mission" in that the administration and faculty understood it well and were "really striving to incorporate it into their teaching" (Karen, interview, 11/17/14). For Karen, teaching in a context that reflected this philosophy reinforced her conceptions of differentiation and was strongly supportive of her implementation of the model during the first year of her practice:

Being in a school that supports differentiation, that knows differentiation, is huge. I can't imagine trying to do this in a school that doesn't support it or isn't flexible and lets you do what you need to do, and then also knows about differentiation. Some people might be like, What are you doing? (interview, 12/08/14)

At Newland Academy, Karen worked with colleagues who had deep knowledge of and belief in differentiation and within a school structure that provided the flexibility for teachers to be able to do it. Thus, on one level, the context in which Karen implemented differentiation reflected an intersection of understanding of Tomlinson's model and structural flexibility. This flexibility included autonomy over her curriculum and instruction and a somewhat flexible daily schedule so that she could lengthen or shorten 
lessons. It also allowed her to meet student needs in slightly unconventional ways.

Karen explained:

Just like little ways, like one of my students who sometimes struggles coming into the room, the fact that I can take him on a walk around the block when the kids go to music so he can calm down, just little things like that. His mom later wrote me an email like we're so glad to be at a school that is so supportive and understanding and responsive to his needs. (Karen, interview, 12/08/14)

In this way, the school's philosophy was realized in its teachers' daily practice.

Administration. Beth described her own approach to leading her employees as a differentiated one (Beth, interview, 12/11/14). During teacher orientation in August, she presented information to the faculty through a variety of formats using a number of different resources and then explained how she made decisions about presenting the information based on her knowledge of the teachers' experiences and approaches to learning (Karen, interview, 9/01/14). Beth worked with her teachers in the same way she would have worked with students. She explained:

So there isn't one way that I support them, in the same way that every year first grade is different depending on the batch of kids you get. But I would say the thing that is consistent is there's time once a week where we always reflect. The same way the teachers are kid-watchers, I'm kind of a teacher-watcher. (Beth, interview, 12/11/14).

Beth usually walked through Karen's classroom several times a day as she led prospective parents on tours (observations, 9/29/14, 10/16/14, 11/17/14, 12/08/14) and felt that she was aware of how each teacher's practice was going (Beth, interview, 12/11/14).

While Karen found Beth to be supportive of her efforts to implement differentiation in general ways, Karen did not seem to feel that Beth was as connected to Karen's actual teaching practice as Beth did. Karen said Beth was not fully aware of her 
daily instruction and wished that there were opportunities to be observed by the administration and to receive feedback on her teaching. Karen explained, "They're telling me, 'Don't be so hard on yourself. You're doing great.' And I'm like, but you don't know what I'm doing. Like, I love the positive reinforcement, but you don't really know what I'm doing in my classroom" (Karen, interview, 11/17/14). By the end of the fall, Karen had come to see this as a contextual factor that challenged her implementation of differentiation, saying that she wanted to be observed by "someone to tell me how I'm doing and what I can work on" (Karen, interview, 12/08/14). Karen would have liked to receive this support from the administration or from her mentor.

Mentor. Before the school year began, Karen was assigned a mentor teacher, an upper elementary teacher with many years of experience who had been at Newland Academy since its opening (Karen, interview, 9/01/14; Beth, interview, 12/11/14). Beth described Newland's approach to mentoring new teachers as involving substantial support: "[T]he key for us with the coaching is making sure that I have them with a veteran teacher who will say, 'Hey, I felt this way too. I felt this way yesterday. It just didn't sink me because I know that's normal'” (Beth, interview, 12/11/14). It seemed Beth envisioned Karen's mentor assuming a coaching role, providing both instructional and emotional support. At the beginning of the year, Karen expected to meet weekly with her mentor (Karen, interview, 9/01/14), but these weekly meetings did not occur. Instead, Karen sought out her mentor only when she had a specific question (Karen, interview, 11/17/14). She explained: “I know they're always there if I have a question or any problem, and it's not that I don't feel comfortable going to them with things, but I kind of feel they're not my best resource" (Karen, interview, 11/17/14). On one 
occasion, Karen had approached her mentor for advice about how to structure her math center rotations and her mentor had explained how she structures her math time in her classroom, but Karen did not seem to find the information useful (Karen, interview, 11/17/14). At the end of the fall, Karen concluded that her interactions with her mentor teacher had played no role in supporting her implementation of differentiation (Karen, interview, 12/08/14).

Elementary team. At Newland Academy, the elementary school includes kindergarten through fourth grade. At the beginning of the year, Karen believed that she would meet with the other elementary grades teachers every Wednesday to plan curriculum collaboratively as a team (Karen, interview, 9/01). Beth also mentioned that, on Wednesdays, "the teachers have a one to two hour planning block together where they can work on hashing out strategies, brainstorming what's going right, what's going wrong, come up with thematic teaching, whatever it is" (Beth, interview, 12/11/14).

In the end, although Karen would sometimes seek out more experienced teachers on her team to ask questions, the collaborative planning sessions Karen had envisioned in August did not occur during the fall. When I asked Karen about the kinds of supports she received from the other members of her team, she responded:

I wish they were a little bit more helpful. And I don't think it's anything about them personally. And it's probably a bit of me too. I don't know what to ask for, or how to ask. Sometimes I'm like, I don't know what I need help with, but I know I need help. (Karen, interview, 11/17/14)

Without regular opportunities to talk about her practice with colleagues by "hashing out strategies" and "brainstorming what's going right and what's going wrong," Karen had difficulty recognizing what she needed help with. It seemed that some support was 
available to Karen from her administration, mentor, and colleagues, but it was incumbent upon her to know what support to ask for.

Ultimately, Karen identified a lack of regular collaboration and planning time with her teammates as a significant challenge to her implementation of differentiation. She believed that "time to talk out our ideas, where I don't feel like I'm impeding on someone else's time that they need for their own room and their own planning time" would have helped her to problem-solve and gain proficiency in her attempts to meet diverse student needs (Karen, interview, 12/08/14).

Karen's students. In addition to Newland Academy's culture of practice, Karen believed that the characteristics of the 17 students in her class served as contextual factors that influenced the ways in which her conceptions of differentiation informed her practice.

Karen enjoyed her students. "I think they're great kids!" she commented during an interview (11/17/14). She felt fortunate that her students brought to her classroom a number of experiences and traits that she felt supported her implementation of differentiation. Because all of her students had attended preschool, they began kindergarten accustomed to the structure of school and prepared to follow instructions and routines in the classroom (interviews, 9/29/14, 11/17/14, 12/08/14). As the fall progressed, Karen came to view her students as a group that worked well independently, had no difficulty understanding her expectations for their behavior, and was able to transition between activities with minimal prompting or reminders (interview, 11/17/14, 12/08/14). Taken as a whole, these student characteristics formed a solid foundation for building a classroom management system that supported differentiated independent work. 
The only aspect of the class that Karen felt challenged her implementation of differentiation was what she perceived to be an unusually wide range of readiness in their reading and math skills, as mentioned above in the Modification of Instruction section. "Oh my gosh!," she exclaimed during an interview as she described the breadth of this range, "I wish you were closer together!" (interview, 11/17/14). I asked Karen whether modification of instruction in her practice would have been any different if her class had reflected a narrower range of readiness. She believed, if that were the case, she would not differentiate more, but she would differentiate better:

I think the quality would be different, because if you have a smaller range, I think you have more time to focus on each [task]. Just when they're so spread out, it's like they're doing completely different things.... Not being so spread apart, maybe there wouldn't be such huge differences in what they're learning. Like in reading, some are reading Magic Treehouse chapter books, and some are just memorizing poems right now who don't know all the letters and letter sounds. (interview, $12 / 08 / 14)$

Karen believed that curriculum in which she designed multiple versions of one thoughtfully considered task would be of higher quality than planning significantly different tasks for students at very different readiness levels. She felt that the range of readiness in her classroom would have been even wider and more challenging if her students had not all attended preschool. "At least I know they're all starting off knowing how to write their names," she concluded (interview, 12/08/14).

Nature of position at Newland. The last set of contextual factors related to Karen's school with a connection to her implementation of differentiation involved the nature of her position as the kindergarten teacher at Newland Academy. First, Karen believed that having an assistant supported her implementation of differentiation. On a day when Susan was absent, Karen reflected: 
Having an assistant is a huge help because it gives me another set of eyes, another set of hands, so that [students] don't have to be doing something independently. Like it was eye-opening today to have [a group that normally worked with Susan] sitting beside me for Word Study, because I was like, realistically, what would I do if I did not have an assistant? (interview, 11/17/14)

Karen felt that Susan's presence in the classroom made group work possible in that Susan could monitor the work of other groups and ensure that students did not interrupt Karen while she was working with another group.

Yet Karen also saw having an assistant who had no formal training in education to be a minor challenge to her implementation of differentiation (interview, 12/08/14). Karen explained that this was because she felt uncertain as to how much direction she should give Susan about how to teach something and whether she should communicate this direction through discussion or a formal lesson plan (interview, 12/08/14). Although Karen only identified concerns about how to guide Susan's instructional practice as a factor that challenged the implementation of differentiation, Susan's interactions with the students may also have detracted from the classroom leaning environment.

During an observation in December, when Susan had assumed responsibility for leading half of the class in math instruction, I noted that she raised her voice in frustration and spoke harshly to students in her group (observation, 12/08/14). For example, when one student left the lesson to go to the restroom with Susan's permission and then returned to the group, she said to him in an accusatory tone of voice, "You missed the problem" after she had just explained a math problem to the group, and she did not reexplain it for the boy (observation, 12/08/14). At one point during the lesson when Susan raised her voice particularly loudly, Karen looked up at Susan, who had her back to Karen. Karen frowned, furrowed her brow, and shook her head slightly from side to 
side (observation, 12/08/14), as though silently objecting to this behavior. Although Susan's presence may have supported structures for group work and learning centers, it may also have detracted from the type of supportive environment called for by a differentiated approach to instruction.

Another issue that Karen viewed as a minor challenge to implementing differentiation was a lack of technology at Newland. Karen had no Smartboard, audio player or recorder, computer, or other technology in her classroom (observation, 9/27/14). This was a result in part of a belief at Newland that technology often takes the place of high quality, engaging curriculum, when it should simply be a tool to deliver that curriculum (Karen, interview, 12/08/14). At the end of the fall, Karen purchased a projector and document camera with her own money to make sharing instructions with students easier (interview, 12/08/14).

The most significant challenge to implementing differentiation Karen faced from contextual factors involving her position at the school was the fact that she was the only kindergarten teacher (interview, 9/29/14, 11/17/14, 12/08/14). Karen described:

Not having someone else who's teaching kindergarten is huge. I didn't realize how big it was going to be, but now, I just realize that's a huge challenge - not having somebody who understands, not that they [other teachers] don't understand my grade level, but maybe someone who has never taught at this grade level or who maybe is teaching the same curriculum that I am can say, "Oh, this is working for me." (interview, 11/17/14)

Karen wished strongly for "a sounding board to bounce ideas off of who knows what it will look like in kindergarten" (interview, 12/08/14). Without a teaching partner at her grade level, a team of elementary grades teachers who met regularly to collaborate in planning and trouble-shooting, or structured support from mentor or administrator to offer feedback, Karen reflected, "Sometimes I feel a little bit alone. Like I know I can 
always ask for help, but I don't know that I'm going to get the help that I really need" (interview, 12/08/14). Because Karen was not confident that the help she needed would come from within Newland Academy, she turned to resources from outside the school for support.

External resources. While most of the contextual factors related to Karen's conceptions of differentiated instruction and related practice involved Newland Academy, one set of these factors involved supports outside of the school. "I just have sought out... other resources that have been more helpful outside of the school," she explained (interview, 11/17/14). When Karen felt she needed support in terms of her knowledge and skills underlying differentiation, she turned to individuals from other contexts in which she had learned about some aspect of the model. When she was uncertain about how to structure learning center rotations in math and how to identify progressions of skills at varied readiness levels, she contacted two of her former instructors from graduate school for help, one of whom advised her to make significant changes to her instructional framework for math (interviews, 9/29/14, 11/17/14, 12/08/14). When she faced similar challenges in designing her literacy instruction, she contacted a third instructor from her graduate experiences (interview, 11/17/14) and also relied heavily on the advice of a reading specialist she had met when assisting at a reading camp (interviews, 9/01/14, 9/29/14). This reading specialist was an experienced public school kindergarten teacher, and she gave Karen guidance on structures for stations through the Daily 5 framework, instructional strategies, and identifying the skills of emergent versus beginning readers (interviews, 9/01/14, 9/29/14). Karen contacted other acquaintances who were public school kindergarten teachers to brainstorm ideas, 
but found these conversations not to be particularly helpful since their students generally had significantly lower readiness levels than Karen's students, and these teachers worked within an inflexible mandated curriculum (interview, 11/17/14). Last, Karen searched the internet for blogs of other kindergarten teachers who were differentiating, reading about how to structure learning centers and ways to organize materials for young students to access efficiently (interview, 10/16/14). During a three month span, Karen contacted at least six individuals outside of her school for support and gathered ideas from multiple kindergarten teachers' websites.

Karen attributed the need for the support of these external resources directly to her attempts to put her knowledge and beliefs about differentiation into practice in her classroom. She described:

I would say because of differentiation, that's why I'm having a hard time working this out. Because I need to differentiate, I need to find a way to do it, so that's where it stems from. If I didn't care about differentiation, and I just taught all kids the same thing, I would have no problem. I'd be like, OK, we'll do it whole group, and we'd break out into games and practice even though you already know how to do this. (interview, 9/29/14)

Karen's willingness to pursue so many resources outside of her school reflected her strong determination to improve the ways in which she identified and responded to diverse student needs in her classroom.

2(c) Summary and factors with greatest influence. The culture of practice at Newland was grounded in a philosophy of differentiating instruction to meet the needs of all learners and a growth mindset for students and teachers. While some support was available to Karen as a first year teacher from her administration, mentor, and colleagues, it was incumbent upon Karen to seek help out and to know what support to ask for. Even if she did so, Karen was not certain that she would get the help she needed from these 
resources. Being the only kindergarten teacher at her school posed a significant challenge to Karen's implementation of differentiation. Without a teaching partner at her grade level, a team of elementary grades teachers who met regularly to collaborate in planning and trouble-shooting, or structured support from a mentor or administrator to offer feedback, Karen felt somewhat alone. This led her to seek help from numerous sources outside of Newland.

Karen's students brought to her classroom a number of experiences and traits that supported her implementation of differentiation. They began kindergarten accustomed to the structure of school and prepared to follow instructions and routines in the classroom, and they executed classroom procedures smoothly during the fall. These student characteristics formed a solid foundation for building a classroom management system that supported differentiated independent work. However, Karen viewed what she perceived to be an usually wide range of readiness in her students as a challenge to implementing differentiation. She believed that curriculum in which she designed multiple versions of one thoughtfully considered task would be of higher quality than planning significantly different tasks for students at very different readiness levels.

Factors with greatest influence. During our interview in December 2014, I asked Karen to reflect on the relationship between her implementation of differentiation and many factors involving Newland's culture of practice, the nature of her position as its kindergarten teacher, and the students in her class that she had mentioned in previous interviews and reflections (see Appendix H). Karen considered each of these factors and classified them as having supported her implementation of differentiation, challenged her 
implementation, or played no role in her implementation, or she indicated that she was uncertain as to whether it was related to her implementation.

After she had explained her responses to all factors, I asked Karen to consider each factor she had characterized as having influenced her implementation, either by supporting or challenging it, and to identify the three factors that had been the most influential. She identified four. In descending order of importance, they were: (a) the wide range of readiness reflected in her students; (b) her growth mindset despite the “steep learning curve" of the first year (interview, 11/17/14), reflected in her willingness to ask for support from a variety of resources beyond her school; (c) Newland's culture grounded in the philosophy and practice of differentiation; and (d) not having opportunities for structured collaboration, feedback, and support from more experienced colleagues, her mentor, or her administration (interview, 12/08/14). Karen viewed her students' readiness range and a lack of supportive interactions with colleagues as significantly challenging her implementation, while she viewed her growth mindset and Newland's culture of practice as significantly supporting her implementation.

As a preservice and first year teacher, Karen remained optimistic about the potential for effective differentiation in her teaching practice that responded to the needs of her students in proactive and meaningful ways. In the fall, she balanced satisfaction with the knowledge she was doing her best with her determination that she would do even better in the future. Her growth mindset was apparent through her relentless seeking of solutions to challenges and sources of support. 


\section{CHAPTER FIVE}

\section{FINDINGS FOR SECOND PARTICIPANT}

This chapter presents findings from a longitudinal case study of Nicole across Phases 1 and 2 of the larger study. Again, findings are presented in response to each of the five focal research questions. Findings for both participants are synthesized in the following chapter.

\section{Nicole's Phase 1 Experiences}

At the beginning of the Differentiating Instruction course in January 2014, Nicole was a 22 year old full-time student in the five-year bachelor/master of teaching (B/MT) program. During Phase 1 of the study in spring 2014, Nicole was in her last semester of coursework, having just completed a semester of full-time student teaching in a third grade classroom in fall 2013. She sought an elementary education (K-6) endorsement. Nicole is a Caucasian female.

\section{Research Question 1(a) Part One: What were Nicole's conceptions of the model when she first entered the course?}

Nicole entered the course with an existing understanding of differentiated instruction developed during her prior teacher education experiences, although she noted, "I think that my understanding of differentiation is still pretty basic, and that's the big part of why I took the class" (interview, 1/27/14). She described her initial conceptions

of differentiation by saying it occurs when "the top group will do this conceptual activity, 
and the middle group will do something a little more concrete, and then the lowest group will do something really concrete" (interview, 1/27/14). When I asked Nicole to provide an example of differentiation in action, she responded, “I don't know, 'cause I don't know if I've ever really done it well" (interview, 1/27/14). She ultimately gave the example of her cooperating instructor's use of Word Study during student teaching in which students practiced spelling with different words while "grouped by whatever feature they were on," with some words being "much more challenging" than others (interview, 1/27/14). Despite offering this specific example, Nicole concluded our first interview about differentiated instruction by telling me, "I have no idea what I'm talking about. I don't know what it looks like" (interview, 1/27/14).

In April, Nicole was again prompted by a reflection question to consider the conceptions of differentiated instruction with which she had started the course. In retrospect, she described:

Before the course began, I exclusively thought of differentiation as a strategy, rather than a philosophy. I thought of differentiation as something I could read enough books on and learn how to "do." The lesson plan template we use in elementary education has a tiny box at the end asking for how we differentiated. I expected, therefore, that this box could be filled with a couple of bullet points so I could 'check the box' that I had differentiated that day. (reflection, 4/06/14)

Nicole's comments suggest that she began the course conceptualizing differentiation as a box to check off representing another strategy to add on to existing instructional practices, rather than a holistic approach to instruction.

\section{Research Question 1(a) Part Two: How did Nicole's conceptions change during the} course? 
As the course progressed and she gained new knowledge of differentiated instruction, Nicole (a) deepened her conceptions of the model, (b) resolved her own misconceptions about its purpose and function, (c) developed understandings of specific model components, (d) identified interdependent relationships among model components, and (e) anticipated a role for differentiation in her future practice in light of her beliefs about its potential efficacy.

Nicole came to understand differentiation as a framework for thinking about instruction that meets all students where they are, or "a lens through which you need to choose to view teaching — one that will have you reflecting, bettering, and redoing a lot of your work over and over again as the needs of your students change" (book synthesis assignment, 2/25/14). She characterized this approach to instruction as fluid based on evidence gathered from students about their needs, and as "far more complex than [she] had given it credit for" (reflection, 4/06/14).

Resolving misconceptions. Almost all of Nicole's explanations of her evolving conceptions of differentiation during the spring involved four key misconceptions about the model that she recognized and corrected. One she identified at the beginning of the course, and two others she identified later in the spring. The fourth misconception she had corrected before the course began.

Differentiation is not synonymous with individualized instruction. Nicole had first been introduced to the concept of differentiated instruction several years before during an introduction to teaching course. One of Tomlinson's texts was an assigned reading for the class. It was at this point that Nicole developed the "biggest" 
misconception about the model she ever held: that differentiation meant individualizing instruction for every learner (interview, 5/01/14). She explained:

When I was first introduced to differentiation, I was very overwhelmed by it, very much like, you essentially want me to individualize? And I think that's a common misconception. You're expecting me to literally individualize lesson plans for 30-some students in my class? (interview, 1/27/14)

After reading the assigned text and participating in course discussions about the model, Nicole came to believe that, in a differentiated classroom, a teacher would write an individual lesson plan for each student. Her response to this idea was to feel overwhelmed and to consider the strategy an impossibility.

Nicole reported that, in the time between taking this introductory course and beginning the Differentiating Instruction course, she had corrected this misconception. She could not identify a particular moment, experience, or course in which this had occurred, feeling instead that the shift in her thinking about this issue had been gradual. However, she did note that her recently developed understanding that differentiation does not mean individualized instruction was "really reinforced" by the new knowledge about the model she gained during the Differentiating Instruction course (interview, 5/01/14).

Differentiation is a philosophy. Nicole's most significant misconception about differentiation that changed during the Differentiating Instruction course involved coming to see the model as a philosophy "or mindset to view teaching through" (interview, 2/14/14), rather than a set of strategies. Before the course began, Nicole completed a reflection for the Differentiating Instruction course in which she related that what she hoped to gain from the class was a set of strategies for differentiating in her future practice (reflection, 1/10/14). Nicole ultimately came to see her expectations that learning to differentiate instruction meant learning a key set of specific strategies as 
"mental roadblocks" or obstacles to her developing conceptions of differentiation that had to be overcome (reflection 2/14/14). She explained, "Once I stopped expecting a set of instructions telling me explicitly how and what to do and not do, I was able to better appreciate the fundamental principles of differentiation and how they work together in a classroom" (reflection, 2/14/14). For Nicole, this reflected a challenging fundamental shift in her conceptualization of differentiation, from checklist to framework:

It's so easy to want to think about it in concrete terms. Well, what strategies is it? What does it look like? What are you doing? And really you can't ever be doing the exact same thing every single day every single year and be differentiating because it's so fluid based on who's in your class and even based on the next day, you know, two weeks ago, they were here, but now they're not, so now we need to totally change what we were thinking about and do something else. So I like calling it a framework or a way of thinking about it - like a mental approach to instruction, I guess, that can be supported with evidence but is not necessarily a list of, do this, this, this, and this.... just want to really conceptualize the idea that it's a framework, not a checklist.... So a way of thinking about how I approach instruction, as opposed to, if you do this and this over the course of a day, then you have differentiated. Congratulations. (interview, 1/27/14)

Nicole recognized that conceptualizing differentiation as a framework for instruction rather than a checklist of strategies represented a significant change in thought that would require time to fully develop in her. Toward the end of the course, she reflected on what she called "the undoing of a thought" she had held for a long time as she considered what had been involved in correcting her misconception (reflection, 4/06/14). She described this "undoing" process as a gradual one, attributing the measured pace of her shift in thinking to having held the misconception for a long time, thoughtfully replacing one big idea with another big idea, and receiving new knowledge about differentiation through a course that was "designed to build upon itself each week," step by step (reflection, 4/06/14). While Nicole characterized her understanding that differentiation is a set of strategies as the most significant misconception she addressed during the course, she also 
identified two misconceptions involving two related principles of differentiation.

\section{Differentiation involves teaching up, not watering down.}

This idea, like, the top is doing the most conceptual, the middle group is doing something that's more concrete, and the lowest group is doing something that's basic, is not actually what I say good differentiation is any more. So I was like, oh wow! (Nicole, interview, 5/01/14)

Nicole entered the course with the understanding that differentiation by readiness called for higher readiness students to engage with conceptually complex material and lower readiness students to do rote work. During the Differentiating Instruction course, she gained familiarity with the concept of "teaching up" (Tomlinson \& Javius, 2012). She explained how she corrected her misconception:

I've gotten a much better sense of like the teaching up idea, that they should all be working with the same conceptual ideas-yes, your middle and lower groups will need different levels of scaffolding, but thinking about it in terms of they should all have the same KUDs is really helpful, and I know that something that helped me when I thought about it was planning the top group's activity first, and then being like, how can I have the middle and lowest groups basically doing that, just with like, what other supports am I going to have to add in? And so I think that's what I would call good teaching up. (interview, 5/01/14)

During the course, Nicole gained new knowledge of this key aspect of high quality curriculum in a differentiated classroom. Through assignments in the course, she practiced creating lessons in which she first designed conceptually rich and engaging tasks for higher readiness learners and then designed scaffolded versions of those tasks for lower readiness students so that they could access the same rich tasks.

This was a different process than Nicole had used in the past to address varied readiness levels in the same lesson. Previously, she had first designed rote tasks for lower readiness learners and then struggled to create related enrichment experiences for higher readiness learners. She described: 
I think it's natural to say, "These are my lowest students. What are they going to be able to do? Gosh they're not going to be able to do anything, and so what little thing can I plan, and then how can I make it more and more enriched for my top students?" And I think that that is actually more difficult, because I've always run into challenges there of, I don't know how to extend this any further. Whereas if you come up with something that's really meaningful and enriching to begin with, I guess it's easier to me to think of it in terms of how to build them up to here, rather than how do I start at the bottom and build all the way up here? (interview, $5 / 01 / 14)$

Understanding the concept of teaching up and applying it to her own work produced a significant reaction in Nicole, who exclaimed "oh wow!" as she described her response during an interview (5/01/14). She then related her new understanding of teaching up to the last misconception she identified during the spring.

Differentiation means all learners are challenged. Nicole entered the

Differentiating Instruction course believing that, in a differentiated classroom, students of different readiness levels would work with different learning goals or content and would not all be equally challenged. She described this as an unconscious belief she did not realize she held until Tomlinson discussed the importance of all students working toward the same high quality learning goals in different ways during the course (interview, 5/01/14). Nicole recalled a particular moment in class when she heard this message and recognized that, in her past practice, she had not followed this principle. She connected this idea to a second principle involving fairness in a differentiated classroom:

And I think it's also some of the source for issues with differentiation because that's where people get the idea that it's not fair-well, my kid has to do harder work because they just happen to be better at whatever, whereas that kid gets to do easier work, and if you really understand what differentiation should be and you're practicing it appropriately, that's not actually true. The learning goals are the same for everybody and it just has to do with the level of support. (interview, $5 / 01 / 14)$ 
During the course, Nicole came to understand the principle that fairness in a differentiated classroom means each student is equally challenged as they work toward the same learning goals in different ways. Lower readiness students do not work with lesser content or shallower objectives; instead, they receive different support as they are challenged to grow.

Developing understandings of model components. Throughout the course, as Nicole encountered information about the supportive learning environment, high quality curriculum, ongoing assessment, modification of instruction, and leading students and flexibly managing routines in a differentiated classroom, she considered this information in light of her existing knowledge and beliefs about that component of teaching and learning.

Learning environment. Nicole did not feel that her understanding of classroom learning environment changed during the course, but she believed her new knowledge of differentiation "further emphasized" the importance of this element of the classroom (interview, 2/24/14). Nicole conceptualized a learning environment as the ethos, culture, or mood felt by the teacher and the students, and "that somebody who walks in can feel and grasp (interview, 2/24/14). She saw a positive learning environment as a precursor to meaningful learning and aspired to create an environment in her future classroom reflecting that "everyone is good at something that is essential to our daily functions in the classroom" (reflection, 2/02/14).

What did change for Nicole during the course were her knowledge about the relationship between learning environment and modifying instruction and her beliefs about the importance of the learning environment in a differentiated classroom. Nicole 
felt that a supportive environment was a non-negotiable precursor to differentiating, and that without it, "your differentiation is just meaningless" (interview, 2/24/14).

According to Nicole, at the heart of a supportive learning environment is the core value of respect. Nicole believed that, in a differentiated classroom, not only do the teacher and student need to respect each other and the students need to respect their peers, but the students need to respect differentiation itself:

[T]he respect thing is really important. I know... a lot about taking the time, especially, again, at the elementary level, too, with students and having them understand that you're not all gonna be doing the same thing at the same time. Your product might look different than his product. If you're in-if the learning environment is negative, then I think that you're not gonna have students who respect that. You're gonna have students who either choose to, I guess in the best sense, just question it too much for it to be effective. In the absolute worst sense, it leads to bullying or just all the bad things that can happen when students don't respect each other. I would say that's, I guess, an important way that learning environment plays into differentiation. (interview, 2/24/14)

Nicole saw a connection between students having respect for their peers and having respect for the differentiated work their peers might be completing. She came to view a respectful learning environment as a foundational aspect of a differentiated approach to instruction.

Curriculum. Nicole reported that her conceptions of high quality curriculum and its relationship to differentiation evolved during the spring, saying, "I think it's changed in that I don't know that I used to think about it as much" (interview, 2/24/14). She explained that, before the Differentiating Instruction course, she trusted that curriculum she encountered in a school, such as a district pacing guide or published literacy materials, would be of high quality. "I would have just been like, 'Oh, it's somebody else's job to worry about that.' Just assumed that they wouldn't have anything that wasn't high quality" (interview, 2/24/14). The Differentiating Instruction course 
introduced Nicole to the idea that not all curriculum, whether published or created by the teacher, was of high quality.

Based on the knowledge she gained from the course, Nicole defined high quality curriculum as having explicit goals, being focused on essential understandings, and being designed to teach up (reflection, 2/14/14; interviews, 2/24/14, 5/01/14). First, she described high quality curriculum as clear curriculum in that teachers, administrators, and students would all have clarity about what students were expected to know, understand, and be able to do as a result. While Nicole believed it should also be engaging, be innovative, and integrate technology as appropriate, "the bedrock of it is always, I think, gonna be, it needs to know where it's going" (interview, 2/24/14). Next, Nicole saw curriculum of quality as leading students to gaining "deep understanding...and making meaning in a way that's transferable. That they're not just learning it to learn content and to cover it. They're actually deeply learning stuff' (interview, 2/24/14). Nicole viewed targeting big ideas rather than superficial learning of facts as a foundational aspect of differentiation, noting, "[I]f you start with trash and you differentiate that's great, but you're just differentiating more trash" (interview, 2/24/14). Last, Nicole considered the principle of teaching up, discussed above in the Resolving Misconceptions section, to be critical for high quality curriculum. She anticipated that teaching up would present a significant challenge when she attempted it in her future practice, in part because she believed she had never seen it done effectively and therefore had no model to consider (reflection, 2/14/14).

Assessment. Nicole felt that her understanding of the purpose and nature of ongoing assessment did not change significantly during the Differentiating Instruction 
course. She attributed this to knowledge she gained during previous coursework and student teaching that aligned with the treatment of assessment in Tomlinson's model of differentiation (interview, 4/10/14). The one aspect of assessment that Nicole did come to view differently due to her experiences in the course involved the use of pre- and formative assessment. Before the course, Nicole believed that it was necessary to grade all formative assessment. As she studied differentiation during the spring, she came to see the primary functions of this type of assessment as informing the teacher's instruction and providing feedback to students on their growth, rather than as "something for the grade book" (interview, 4/10/14). She also came to believe in the importance of using assessment data to inform flexible grouping decisions for tasks tiered by readiness. Nicole explained:

I realized that if I wanted to tier appropriately that I would need to use preassessment data specific to the upcoming unit, not just what I already thought I knew about the students. This was an a-ha moment, because even though it made sense as soon as I realized it, I realized I hadn't ever seen that really modeled for me, and so it had not occurred to me until it was explicitly talked about in class. (interview, 4/06/14)

Nicole's newly developed conceptions of formative assessment informed her understanding of modifying instruction by readiness.

Modification of instruction. One of the key areas in which Nicole reported gaining new knowledge about differentiating instruction during the course involved modifying instruction based on differences in student readiness, interest, and learning profile.

Responding to student readiness. Nicole defined the concept of readiness as "the point at which a student arrives at the material" (interview, 2/24/14). She described a student's readiness as differing from topic to topic or skill to skill and changing over 
time, emphasizing the importance of avoiding assumptions about a student's proficiency level (interview, 2/24/14). She also noted the key role of identifying patterns across students' preassessment data to group students for instruction based on demonstrated readiness (interview, 4/10/14). According to Nicole, the information she gained from the Differentiating Instruction course enhanced her knowledge about responding to student readiness through instruction:

I think that it's changed in that I'm gaining a better sense of what's appropriate. I think that I always had a foggy sense of, yeah, that's something you should do. Now I feel I'm getting a better sense of like, okay. Well, this is - with readiness, we've been talking about tiered lessons or any of the other different ways that you can do it. I think I wouldn't have had - I think it would have just been a nice idea before. Now I feel like I'm in a little bit better position to actually act on it from time to time. (interview, 2/24/14)

Nicole began the course with a belief in the importance of responding to differences in student readiness through her instruction, but with a lack of knowledge of how to use specific strategies to do so. With the knowledge she gained from the course about designing tiered lessons and other responsive approaches, she was later prepared to "act on" her beliefs in a way that she was not before the spring.

Responding to student interest. "I used to see that as the fluffy version of differentiation" (interview, 4/10/14), Nicole responded when I asked about her impressions of modifying instruction by student interest before the course. She continued:

Like readiness was absolutely, if you have a kid who's three grade levels below, you have to deal with that. Learning profile the same, where it's like, if a kid's not gonna process information best in that way, then you need to provide them with some other avenue. Interest was that, "Oh, well, it's nice to do sometimes, to keep the kids happy every now and then." To be like, "Sometimes Miss Nicole lets us choose." I would say that's how it was before.... I'm doing it just because it's like, "Oh, I feel like my approval ratings are dipping." (interview, 4/10/14) 
Based on the knowledge she gained from the course, Nicole came to view modification of instruction by interest as an effective engagement tool that created a sense of student ownership of their learning through choice and that could be "very academic" (interview, 4/10/14). During the course, Nicole practiced designing lesson plans that included modification of instruction both by interest and by learning profile or readiness. Using interest-based differentiation in combination with one of the other approaches "helped elevate interest as a much more valid way" to differentiate in Nicole's mind (interview, 4/10/14).

Responding to student learning profile. Nicole defined learning profile as "how a person prefers to work....how a person works most efficiently," noting that its components are numerous and complex (interview, 4/10/14). She reflected that the way she thought about responding to student learning profile had changed significantly during the Differentiating Instruction course:

I think that I've expanded my view of learning profile to be beyond auditory, visual, kinesthetic learner. To be more intentional than just...I'm gonna slap up the directions, and I'm gonna say them. Which, again, is a valid thing to do, but it's not really high-quality differentiation. It's not a whole lesson. [I'm going to be] more intentional in the lesson planning, providing or designing a learning experience that's directly tied to learning profiles. (interview, 4/10/14)

While Nicole had understood students differed in their preferred approaches to learning and believed in the importance of responding to those differences before the spring, the knowledge she gained from the Differentiating Instruction course allowed her to visualize ways in which she could account for those differences systematically through intentional planning.

Leading students and classroom management. The way Nicole thought about leading students and managing classroom details changed during the Differentiating 
Instruction course and caused a shift in her thinking about how she might lead her future

classroom. This shift created an emotional response in Nicole as she considered its implications. Nicole explained:

I want to be more open to a more leading, less managing type of classroom. I know that something - I mean it still makes me a little bit anxious, but that I'm trying to be more open about — is that idea of students doing a lot of different things. Students just doing independent work, or working in small groups. That is something that, from a management point, makes me a little bit anxious.... What I want to happen is that when I do go into my own classroom, that I'm more open to it, despite the fact that I'm anxious about it. Just try it and do everything that I can to make it work. Set up all the routines and everything so that we have good expectations, and try it. If it doesn't work, hopefully not get discouraged and be like, "I'm never doing this again"....Because I do see it as really valid, but I also recognize that part of myself that's, "I'm gonna lose control of the classroom!" I guess I would say it's changed because I want to be more open to it now. Whereas, I think at the beginning of the course I think I was just very, "Nope, gotta have control of the classroom. Gotta hang on to it. Gotta keep it from devolving into chaos." (interview, 4/10/14)

As she considered the new knowledge she gained during the Differentiating Instruction course about leading and managing a differentiated classroom, Nicole easily recognized her own proclivity for "tight-ship" or “orderly restrictive” (Tomlinson \& Imbeau, 2010, p. 75) management, as well as the conflict between her natural management style and the flexible management needed for students to operate independently within the classroom. By April, Nicole considered herself "more open" to the possibility of a more flexible approach to management, although she felt anxious about trying such an approach in her future practice and losing control of the classroom.

Toward the end of the course, as Nicole reflected on the concepts involved in Tomlinson's model of differentiation about which she had gained the most new knowledge, she noted that her understanding of flexible grouping practices had greatly deepened during the course (reflection, 4/06/14). She entered the course defining the 
term as not putting students in the same groups all the time and saw the practice as "just something nice you do to mix it up and keep the students from 'knowing' who was in the 'top' and 'bottom' groups" (reflection, 4/06/14). She came to see the practice instead as "essential and intentional" for modifying instruction based on readiness, interest, or learning profile using specific assessment data (reflection, 4/06/14).

Identifying relationships among model components. During the course, Nicole's conceptions of differentiated instruction reflected an understanding that the five elements of a differentiated classroom function as an interdependent system. In April, she viewed these elements as playing different roles and having different relationships with one another, but as all being of equal importance. She used the metaphor of the spokes on a wheel to illustrate this idea, saying that if the spokes are not all of equal length, the wheel will not function properly (interview, 4/10/14). At the conclusion of the course, I asked Nicole to reflect again on the interrelationships among the elements of a differentiated classroom by creating a concept map.

Nicole's Phase 1 concept map. During our final Phase 1 interview, I requested that Nicole draw a visual representation of the relationships among the five elements of Tomlinson's model, and I invited her to add any other aspects of a differentiated classroom that might not be encompassed by those elements. I also asked Nicole to think aloud as she considered how to create the representation. Nicole's Phase 1 concept map appears in Figure 3. 


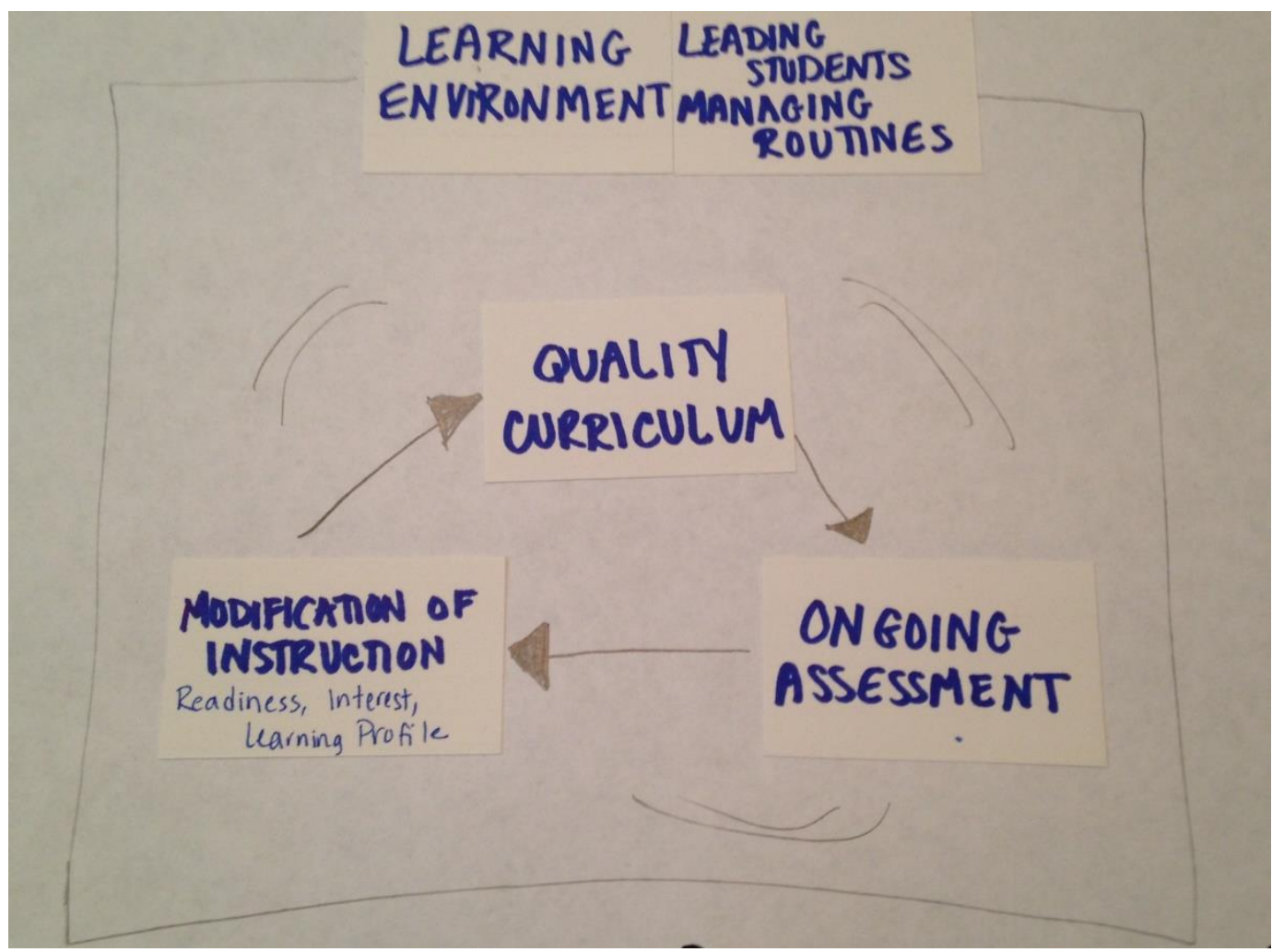

Figure 3. Nicole's first concept map illustrating relationships among the five elements of a differentiated classroom. Created during Phase 1 Interview 4 on May 1, 2014.

Nicole drew a box encompassing most of the page and labeled the box learning environment and leading students and managing routines. She explained that these two elements "encompass everything else" and that "without these two things making the box, the rest of this doesn't really work" (interview, 5/01/14). She placed quality curriculum, ongoing assessment, and modification of instruction inside the box. These three elements were positioned to create an equilateral triangle with quality curriculum at the top (concept map document, 5/01/14). Nicole explained that this was because curriculum was the starting point (interview, 5/01/14). She drew an arrow from quality curriculum to ongoing assessment, an arrow from ongoing assessment to modification of 
instruction, and an arrow from modification of instruction to quality curriculum (concept map document, 5/01/14). According to Nicole, she drew the arrow from curriculum to assessment "because you can't just teach your quality curriculum and trust that it's quality and say, 'Everyone should get it.' You have to check for understanding” (interview, 5/01/14). The arrow from assessment to instruction indicated "based on your assessment results, that's what you need to use to modify your instruction" (interview, 5/01/14). She explained the arrow between assessment and curriculum by saying, "And then it all goes back to your quality curriculum, because once you modify your instruction, you still have to teach it in a quality way, and check for understanding, and modify, so this is supposed to represent an ongoing cycle" (interview, 5/01/14).

While in April Nicole had characterized all of the non-negotiable elements of differentiation as equally important, during this concept mapping activity in May, she believed that, "At the end of the day, the learning environment is the absolute most important thing....The learning environment is the foundation that everything else can build off of" (interview, 5/01/14). She explained that the students feeling safe and respected was a precursor to any other aspect of differentiation occurring in the classroom and therefore construed learning environment to be slightly more important than the other components. Interestingly, Nicole characterized leading students and managing routines as "the embodiment of the learning environment-it's walking the talk" (interview, 5/01/14). She saw the learning environment as being created by the teacher through the ways in which she led the class and managed the details of running the classroom. Nicole used the metaphor of constructing a building to describe this:

If this were an actual foundation in construction, learning environment would be the first layer of concrete that you pour down. And then leading and managing 
would be the layer of concrete that you pour down after the first one dried. So still the foundation, but if you have to pick one to put first, I would say learning environment. (interview, 5/01/14)

When it came to the cycle of curriculum, assessment, and instruction, Nicole viewed the relationships among them represented by arrows on her graphic as equally weighted, but saw quality curriculum as "the most important thing to start with" (interview, 5/01/14). Nicole's concept map reflected her nuanced understanding of how the five elements of a differentiated classroom may function as an interdependent system.

Anticipating future practice. Nicole anticipated that differentiation would be a part of the way she thought about teaching and learning and a part of her practice as a first year teacher. While she held strong aspirations about trying many of the approaches she had learned about during the course, she felt concerned about how she would react if her attempts did not go smoothly (interviews, 1/27/14, 5/01/14).

Nicole spoke in strong and certain terms about the role she envisioned for differentiation in her future classroom:

I want it to influence all of my instructional decisions. I want to have a classroom where differentiation is the thing that we do and not just something that we do occasionally. I want it to be that founding principle that everything else in the classroom is built upon. I definitely want to use what I've learned to work towards creating that positive classroom climate. I want to be able to meet my students at where they come to me, and not say, I'm up here, so you've got to get here. And I want my administration to support me in these efforts. (interview, $5 / 01 / 14)$

Because Nicole recognized that differentiation looks different in every classroom with every group of students, she understood that, as a preservice teacher, she could not fully envision or predict how the instructional philosophy would influence her daily teaching practice with her future students. She therefore described her key goal in taking the Differentiating Instruction course as to develop the skill of recognizing the individual 
learner needs present in her class as quickly as possible so that she could respond to them efficiently (interview, 1/27/14).

Notably, Nicole seemed to consider the biggest challenge she would face in implementing differentiation in her future teaching practice to be her own disappointment with her attempts, or lack of attempts, to differentiate. At the beginning of the course, Nicole explained this concern:

I think the challenge will be knowing that I'm not doing it perfectly, or knowing that I'm not doing it as much as I should be, but also knowing that because it's my first time doing it, I'm doing it as much as I can. So I guess the challenge of knowing that this is the most that I can do because I'm, it's still just my first time doing it. But it's not nearly enough, 'cause it needs, what they deserve is over here, and what I can do is like here. So I think that would be challenging. Just cause I know it's not gonna be feasible for me to expect myself to do differentiation perfectly all day every day all year, even though, in an ideal world, that's what would happen. And it would be great and beautiful and sunshine. But just realistically, again, I'm gonna be like here, exhausted. It's hard, it's hard to think about that first year of teaching. Being like, I'm not gonna be at my best, but like this is still a really important year of school for them. It doesn't really feel fair. (interview, 1/27/14)

Nicole began the semester believing that, as a first year teacher, she would not differentiate instruction as frequently as she wanted to, and when she did differentiate, she would not do it perfectly. These concerns seemed to be sources of discouragement for Nicole.

At the conclusion of the course, although Nicole's conceptions of differentiation had deepened considerably, she still described her concerns about her future practice in remarkably similar terms to those she used at the course's beginning:

I'm afraid that it's going to be, I'm thinking about it, and I'm disappointed with where I'm actually performing, because I just don't think that it's reasonable for me to expect that as a brand-new, first year teacher I'm going to go out next year and practice differentiation perfectly all the time. I don't know that anybody can do that. I'm trying to mentally be prepared for the fact that I'm going to make mistakes in my first year of teaching, and so I'm going to have it in the back of 
my mind and hopefully make mostly good decisions, but I'm sure there will be instances where I realize, that's not quite what I should do. That's not differentiation the way I learned it. That kind of thing. That could come from any number of things - how restricted I feel or don't feel in terms of curriculum I have to get through in a year, or just me planning a lesson and looking back on it afterwards and saying, that's probably not the most respectful tasks I could have designed. How can I do that better? (interview, 5/01/14)

Nicole was exceedingly aware of the fact that, as a first year teacher, she would be unable to differentiate perfectly. The idea that she would fail to meet her own expectations appeared to cause her disappointment, and she responded by attempting to "mentally prepare" for the mistakes she felt she would inevitably make in her practice.

1(a) Summary. Nicole began the course with an understanding of differentiation as another strategy to add on to existing instructional practices, rather than a holistic approach to instruction. She also interpreted the model as calling for higher readiness students to work with abstract, enriching curriculum and lower readiness students to work with concrete, rote tasks. During the course, she gained knowledge of the principles and practices of differentiation and her beliefs about the importance of modifying instruction deepened. She came to see differentiation as a framework, lens, or mindset for thinking about instruction, rather than a set of strategies, and resolved other misconceptions involving challenging all students and teaching up. Nicole reported significant changes in her thinking about curriculum, modification of instruction, and management in a differentiated classroom, and in her understanding of the interdependence of all five elements. She anticipated that differentiation would be a part of the way she thought about teaching and learning and a part of her practice as a first year teacher, but felt concerned about how she would react if her attempts did not go smoothly. 


\section{Research Question 1(b): How did factors external to the course relate to Nicole's conceptions of the model?}

As Nicole made deeper meaning of the philosophy and practice of differentiated instruction throughout the spring semester, she identified previous experiences that played a role in her developing knowledge and beliefs about the model. These factors involved varied experiences in her teacher education program and her personal background.

Personal background factors. As Nicole discussed differentiation during interviews and reflections, she referenced many aspects of her background that she saw as related to her evolving conceptions of the model. These factors included her prior beliefs about teaching and learning that she brought to the course, her own K-12 educational experiences, and other relevant personal characteristics.

Prior beliefs about teaching and learning. Nicole described the foundational principle of the philosophy of teaching and learning she brought to the course as being the idea that every student is capable of learning. Although she had persisted in this belief throughout her teacher education experiences, she did note that it had been challenged when working in difficult field placements in which other teachers reflected a belief that "this kid is just unreachable" (interview, 1/27/14). As a result, Nicole

"adapted" her view of this foundational principle by adding a caveat. She explained:

I think that as much as I want to do everything I possibly can to reach every student, it needs to be a shared learning experience, and there's always more that I can be doing, absolutely, but I think that part of how it's altered is that I've recognized that I am only human, and at the end of the day, I can't make somebody do anything. It's my job to engage them, but they also have to work really hard for it to really work. (interview, 1/27/14) 
As she progressed through her teacher education program, Nicole modified her initial philosophy as she came to believe that all students were capable of learning if they put forth effort to grow in partnership with the teacher. Because of this belief in the capability of all children, Nicole felt confident that she could work effectively with any type of student in the general education population, although she did note that, in an ideal world, she would love to teach students who all had supportive home environments and were working on grade-level (interview, 1/27/14).

Nicole also came to see a teacher's role in the classroom from a more holistic perspective, developing a more "complete picture" of it over time (interview, 4/10/14). At the beginning of her preparation program, she had viewed teachers as having a primarily academic role focused on delivering content to students. By the conclusion of her program, she viewed teaching in a more "holistic, whole child way" (interview, 4/10/14), and she recognized that her coursework and field placements had deepened her understanding of its complexities. "This is way more complex than I gave any of my elementary teachers credit for," she concluded (interview, 4/10/14).

During the course, Nicole appeared to consider thoughtfully the new knowledge she gained about the principles of differentiation and to assess the degree of alignment between these principles and her existing beliefs about teaching and learning. She described the alignment between the two to be tight (interview, 5/01/14). For example, in late January, Nicole already saw a connection between differentiation and her foundational belief that, with effort, all children are capable of learning. She explained, "I think for differentiation to work, you have to truly believe that there is something that can reach every single student, no matter how challenging they've been in the past or 
how little success they've had up to that point" (interview, 1/27/14). By the end of the

course, Nicole had a broader and deeper understanding of the nature of this alignment:

I see a lot of alignment because I have really, over the course of being in this program, developed a philosophy of education and schooling being about the entire child, in terms of deep philosophical beliefs....I want my teaching and curriculum to be about so much more than hard core standards and test prep and all of these things, and I think DI does align really well with that, because at the end of the day, I think it's really about making meaning and writing good Uswhat do you want your students to understand? I think it's about teaching in a way that is meaningful and not something they're going to forget 15 minutes after they take their test. And it is about having them like challenge and like the challenge of learning, which ties in well with the whole child thing because to succeed in life you have to be able to tackle challenges and not just shrink away every time things get difficult. (interview, 5/01/14)

These comments illustrate a connection Nicole recognized between her belief that the role of a teacher involves educating the whole child for success in life, not just on the next test, and a differentiated approach that challenges each learner through high quality curriculum.

While Nicole and I used the term alignment to describe the relationship between her prior beliefs about teaching and learning and the philosophy of differentiation during our interviews, Nicole's comments about this relationship suggest that this term may not fully capture the complexities of this phenomenon. Nicole did not simply conduct a sideby-side comparison of two sets of beliefs as separate, parallel entities and form conclusions about the potential connections between those entities. Instead, the two became intertwined. For example, Nicole wrote in a reflection, "Once I accepted that it was a philosophy and not a set of strategies, it helped me fold the ideals [of differentiation] into what I believe about teaching in a much more fluid way" (reflection, 2/14/14). Thus, this "fluid" process involved Nicole "folding" her new beliefs about teaching related to differentiation into her existing beliefs. Through these experiences, 
Nicole gained greater clarity surrounding those existing beliefs. This became evident to her as she applied for jobs in the spring. She described:

I had been really struggling with my job applications and stuff. They all wanna know is - what's your educational philosophy?.... I was finding it very difficult to answer because I couldn't-I was finding it difficult to express what I thought in a way that was concise and clear and also got at everything that I believe. I found that once I [understood differentiation as a philosophy, not a set of strategies], that idea helped me. When I then went back to try and rewrite my educational philosophy, I was like, "Okay. This all makes a little more sense now. If this is the lens through which I'm gonna view my instruction, then all these other pieces can fall into place." In terms of modifying instruction for student readiness, using ongoing assessment to drive instructional decisions, creating a learning environment that's a safe space and respectful and nurturing and all those things. I just found that everything flowed from that. It became a more concise statement. I was like, “This actually makes sense.” (interview, 2/24/14)

When Nicole adopted differentiation as a lens through which she viewed instruction, her beliefs about various aspects of teaching and learning came together as a coherent whole that "made sense."

This was a semester-long process of clarification for Nicole. She described the beliefs about teaching and learning with which she entered the course as "vague" and not "well-defined" (interview, 5/01/14). The Differentiating Instruction course prompted Nicole to define her beliefs more precisely and gave her the "verbiage" (interview, 5/01/14) to describe what she believed: "It helped me put words to what all those things I was thinking meant....[A] lot of how I [now] think about my teaching philosophy in my head, the words to it have come from being in the class" (interview, 5/01/14). Looking back on her experiences in the course as a first year teacher, Nicole concluded that starting with her existing philosophy of teaching and learning,

And then sticking it into the differentiation model allowed those two things to work together in concert. So then I was like, "Oh great, this [existing philosophy] is something I want to bring to my own classroom, differentiation is something I 
want to bring into my own classroom, and look, they go so nicely together." (interview, 12/09/14)

According to Nicole, the fact that she recognized as a preservice teacher that her existing philosophy and the philosophy of differentiation would "work together in concert" led her to see a role for differentiation in her future practice.

Prior educational experiences. Nicole had attended kindergarten through high school at "resource-rich" public schools in the same wealthy district (interview, 1/27/14). She took advanced and honors courses as a secondary student and viewed herself as successful and hardworking throughout her K-12 experiences, once commenting during a whole-class discussion in the Differentiating Instruction course, "Obviously I cared about my grades because I'm here [at the University of Virginia]" (observation, 4/10/14).

Nicole could not recall having many elementary or secondary experiences that involved differentiated instruction. She did remember being assigned to the highest reading group in elementary school and felt that students in her class were highly aware of the reading readiness of their peers (interview, 4/12/14). Nicole felt that this likely had a discouraging effect on her classmates who were lower readiness readers. She could not recall seeing any instruction during her high school years that was differentiated (interview, 4/12/14). She could, however, think of specific classes in which it was needed when students did not understand the content as presented and struggled to learn it on their own (interview, 4/12/14). Nicole felt that these experiences did not support her developing conceptions of differentiation.

In sixth grade, Nicole did encounter a math teacher who used multiple modes of instruction to support conceptual understanding (interview, 4/12/14). In this class, lesson learning goals were clear to the teacher and to the students. This teacher seemed to have 
an attitude of being willing to try numerous approaches to instruction to support student learning (interview, 4/12/14). Nicole saw this particular middle school experience as enhancing her understanding of differentiation when she thought back on it during the Differentiating Instruction course. She looked to this experience as a positive example of teacher student connections in a supportive learning environment and presenting content through multiple modes of instruction.

During the course, the most critical connection Nicole came to recognize between her K-12 experiences and her developing conceptions of differentiation involved the fact that she viewed herself as "totally a product of fixed mindset classrooms" (reflection, 2/14/14). Because this realization is closely related to Nicole's personal characteristic of mindset, it is discussed in the following section.

Personal characteristics. The personal traits reflected in Nicole's comments appeared to revolve around issues of flexibility, predictability, organization, patience, control, anxiety, and perfection. Nicole seemed keenly aware of how her own reactions to these issues might influence her teaching practice and was consistently introspective throughout the semester.

Nicole described herself as someone who "really likes planning and lists and to be very, very organized" (interview, 2/24/14). She explained that this trait caused "mental dissonance" in her because although her "personality wants it to be this one way," entering teaching requires that a teacher "accept that it's not always gonna be that way....that teaching is messy" (interview, 2/24/14). Nicole explained, "It causes me mental conflict to think about how it seems like so much I cannot plan for. I think to overcome this I need to try and have a more flexible attitude toward teaching" (reflection, 
2/14/14). Nicole recognized the need for flexibility in response to the "messiness" of teaching, but she perceived achieving that flexibility as difficult for someone who instinctively preferred high levels of organization. She described being unable to prepare for every contingency she would face in the classroom as "stressful" (interview, 2/24/14). A specific example of this involved her attitude toward the flexible approach to classroom management present in an effective differentiated classroom. As noted above, while Nicole said she wanted to "be more open to a more leading, less managing type of classroom," the idea of doing so made her "anxious" out of fear that she would lose control of her classroom, with it "devolving into chaos" (interview, 4/10/14).

Recognizing that she would not achieve perfection in differentiation as a first year teacher appeared to cause Nicole disappointment and frustration. She mentioned this fact five times during three interviews, using some variation of the word "perfect" each time (interviews, 1/27/14, 2/24/14, 5/01/14). In fact, Nicole characterized the knowledge that she would not be able to differentiate perfectly during the first year as the primary challenge she anticipated facing when she tried implementing the model in her own practice (interview, 1/27/14). Nicole also noted patience was not a personal characteristic that she had in great supply, saying that she hoped to develop more patience in the classroom (interview, 4/10/14). She described herself as feeling impatient with her class if they worked more slowly than she anticipated, with individual students if they were not trying their best, with the planning process if it took a long time to prepare a differentiated lesson, and with herself as a new teacher (interview, 4/10/14). This lack of patience seemed connected to Nicole's disappointment with her awareness that she would not be able to implement perfect differentiation as a new teacher, as well as to her 
mindset.

Mindset. "The most surprising thing I have learned about differentiation is how much mindset impacts the classroom," Nicole reported (reflection, 2/02/14). While she had heard of the concept of mindset before the course, Nicole had not considered in depth its implications for teaching and for life until she studied it in the context of differentiation. Nicole described her newly developed understanding of mindset as almost revelatory: "I literally, now that I have the words growth and fixed mindset in my head, I see the world in this whole new way. I understand everything around me - that's a broad statement—-but we've talked about how it helps us understand like that's why this person has been acting this way" (interview, 5/01/14).

The primacy of this concept in Nicole's thinking about differentiation and teaching in general was reflected in the frequency and depth of her comments about the topic in course assignments and interviews. Nicole raised the topic, without my specifically asking her about it, in three out of four Phase 1 interviews (1/27/14, 2/24/14, $5 / 01 / 14$ ) and in three out of four written reflections she completed as course assignments $(2 / 02 / 14,2 / 14 / 14,4 / 06 / 14)$. Rather than characterizing herself as having a growth mindset or a fixed mindset, Nicole described herself as having areas in which she did not yet have a growth mindset and areas in which she had a growth mindset but was not yet acting on it (reflection, 2/02/14). She believed that her current mindset status did not support her conceptions of differentiation, but that her experiences in the Differentiating Instruction course in which she was prompted to consider the importance of mindset in the classroom did support her developing conceptions of the model (interview, 12/09/14). 
Nicole attributed the fact that she did not have a fully growth mindset largely to her own K-12 experiences. Nicole described herself as "totally a product of fixed mindset classrooms" and a student who continued to be challenged and grow during her K-12 experiences because she had been classified as one of the "smart' kids" (reflection, 2/14/14). She explained what it meant to be a product of fixed mindset classrooms:

I see tracking as the ultimate manifestation of fixed mindset. I know that....a big reason I was successful in school is because at a very young age enough people told me that I was smart. Just realizing the power of that. What if I hadn't gotten that? Would I be in the same position that I am now? Just realizing the power that an adult has in determining that. Then as a teacher, it would be my responsibility to give that to every student. Not just students like me. Basically, having started at that very young age, I was identified as gifted, whatever that means, and put into gifted enrichment, put into honors classes, put into AP classes. It just continues all the way up. I definitely believed in K-12 school that there were smart kids and there were dumb kids. I was in the class with the smart kids. We were the successful students. We were gonna go on to the UVAs of the world. Some people just weren't. I think that when I say I'm a product of the fixed mindset, I think that the system that encouraged me to keep going into advanced classes and discouraged other people from going into advanced classes just represents the belief that, "Well these students are going to continue to succeed. They're gonna go and go and go. These students are just not." (interview, 2/24/14)

Nicole saw an educational system that, in her view, was predicated on a determination that some learners identified at a young age would be successful and some would not as reflecting a set of beliefs with an inherent fixed mindset. This caused Nicole conflict, because she had fared well in this system. She explained:

That's challenging because obviously that system benefited me. I'm pleased with where I am in life. It's hard to grapple with that, to know that's not the best thing. It's not really the best reason why I necessarily am the way that I am. (interview 2/24/14)

While Nicole seemed to find disquieting the fact that a fixed mindset system in which she had advantages over other students had benefitted her, she was conflicted by the pride she felt in her hard work as a K-12 student that had also contributed to her success, saying, 
"It's difficult to reconcile knowing that that environment ultimately benefitted me, because I am proud of the educational accomplishments I have achieved over the course of my life so far" (reflection, 2/14/14).

Nicole explained that she wanted to have a growth mindset, but that to do so, she would have to work to change her mindset, since "it's difficult to undo 18 years of school that reinforced all day every day that one mindset" (interview, 2/24/14). She ultimately concluded that having been educated in a fixed mindset system featuring tracking and competition among students in advanced courses detracted from the development of her conceptions of differentiated instruction, since it influenced beliefs about teaching and learning that she would have to "undo" to differentiate effectively. Nicole described in a frustrated tone of voice, "Because this K-12 experience is one of the most powerful learning experiences that's contributing to who I am as a person. Because it's many, many, many years of my life" (interview, 12/09/14).

Nicole believed that the nature of her mindset as a teacher would carry significant implications for her students. She explained:

It was surprising to realize how deeply entrenched that model of "the way school is done" is, and to realize how intentional I'm going to have to be about changing that as a teacher, since I cannot change the fact that that's what I know from my classrooms growing up. (reflection, 2/14/14)

Nicole envisioned a number of ways of being "intentional" about demonstrating a growth mindset as a teacher, including regularly assessing how mindset was influencing interactions with students (reflection, 4/06/14), how she thought about her students in terms of their capability (interview, 2/24/14), and keeping a reflective mindset journal to maintain an awareness of areas in which she was not acting on a growth mindset (reflection, 2/02/14). 
Part of Nicole's interest in continuing to examine the status of her mindset while in the classroom was her belief that mindset is inevitably reflected in a teacher's instruction, often in subtle ways (reflection, 4/06/14). She viewed thinking of students in terms of whether or not they were capable as harmful. Nicole explained:

A true growth mindset would mean that I designed instruction that truly gave all my students the best opportunities to succeed, instead of just assuming some of them are 'smart' and some of them are 'not smart.' I want this to impact my practice through me constantly asking myself the hard questions of whether or not my lessons plans, conversations with students, and attitude are reflective of the growth mindset, and if they are not, asking myself what I need to change. (reflection, 4/06/14)

Nicole expressed a concern that she might begin thinking of her students as those who would inevitably perform well on the high stakes test at the end of the year, those who would not, and those in the middle (interview, 2/24/14). She explained that she did not want to label students in this way because she believed it would affect her expectations of students in those groups, which would in turn affect their performance. Her goal, therefore, was "Just having to make sure that my actions are really reflecting the fact that I do think that they can all be successful" (interview, 2/24/14). She viewed this as particularly important when working with students who often had lower readiness, saying she would work to ensure she did not assume that because they needed additional support in some academic areas, they would be unable to be successful in others or would be unable to grow from their starting points (interview, 2/24/14).

Although Nicole spoke often of how mindset could affect interactions with students, she did not reflect on how her mindset influenced her attitudes toward herself and her teaching practice. It seems likely that her strong and unremitting concerns about 
making mistakes with differentiation during her first year of practice and being unable to achieve perfection discussed above can be attributed to a lack of a growth mindset.

Teacher education program factors. Throughout the spring, Nicole noted significant relationships between her evolving conceptions of differentiated instruction and her previous experiences in her teacher education program, including her B/MT coursework in the elementary education endorsement program and her numerous field placements. Some of these experiences supported her developing conceptions, while others detracted from it.

Field experience. During the two and a half years before the study began, Nicole completed six field experiences. In her third and fourth years of the B/MT program, she spent several hours per week in her field placements, with a different placement each semester for a total of four placements over two years. In her third year placements, she observed or provided individual students with one-on-one support. In her fourth year placements, she observed and taught a handful of short lessons. She was placed in first, third, and seventh grade classrooms for these experiences in her third and fourth years. Nicole then student taught fulltime in the fall of her fifth year. She spent half of the semester in a third grade classroom in a local school and half the semester student teaching in the American equivalent of a third grade classroom in Belfast, Northern Ireland.

Initial placements. Nicole did not see much of a relationship between her experiences in placements during her third and fourth years and her conceptions of differentiation. She could not think of an example of differentiated instruction from any of those four placements except one third grade classroom during her fourth year, where 
she "some level of differentiation going on" through the use of guided reading groups (interview, 2/24/14). Nicole explained that, because she was only in these classrooms for an hour and a half at the same time each week, she did not see a variety of instructional strategies used across different strategies. Although she felt that these experiences might have shaped her understanding in some way, she said she could not articulate what that might be (interview, 2/24/14).

Local student teaching. From August to mid-October 2013, Nicole student taught in the third grade classroom of a local school. In spring 2014, she characterized this overall experience as very positive (interviews, 1/27/14, 2/24/14), describing the context of the school as having a positive culture in which teachers and students were focused on doing their best (interview, 2/24/14). Nicole viewed the greatest strengths of her CI as establishing community and routines well at the beginning of the year and caring deeply for her students (interview, 2/24/14). As an example of how her CI built community among the students and teachers, Nicole described an activity in which the CI asked students to make a "Me Bag" filled with four small items that revealed something about themselves. Students shared their items with a classmate, and later, the classmate introduced that student and their items to the class. Nicole described, "I saw her expertly guide discussion after each of them...that helped the students begin to see the very unique strengths that each person brought to the class" (reflection, 2/02/14).

During our February interview, Nicole characterized her CI as "differentiating well" (interview, 2/24/14), but she also explained:

I didn't necessarily see a ton. I didn't see a tiered lesson or things like that. Just definitely made me more open to the idea of stations and working groups and things like that....I think it's sometimes scary to think about managing that. Like, "Oh, how can I trust the students to do all this stuff independently?"....Letting me 
get a feel for that in realizing it's actually okay. It's gonna be fine if they're all working at different stations, and I'm not the controller of instruction. (interview, 2/24/14)

In a February reflection, Nicole also noted that she had "never seen [differentiation] truly done well in an actual classroom setting" (2/14/14). Considering all of these statements together, Nicole's experiences with her CI appeared to influence her understanding of establishing a supportive learning environment and managing classroom details flexibly, but did not involve the elements of high quality curriculum, ongoing assessment, and modified instruction based on variance in student needs. While her CI provided a number of effective examples of some elements of Tomlinson's model of differentiated instruction, Nicole did not see all elements working together interdependently in this context.

During her student teaching experience, Nicole also interacted periodically with a university supervisor. Differentiation was not a topic Nicole talked to her supervisor about often; it only came up if there was an issue with something Nicole had included in her lesson plan. She described, "So there's a part of the lesson plan template that, at the very bottom, it's this little box, elements for differentiation. I know that I threw... some parts of it at her: Do you think this is okay?" (interview, 4/10/14). Although Nicole did not recall the specifics of any of those conversations, she did recall that her supervisor had approved the inclusion of one "element for differentiation" on a lesson plan involving color coding some materials to make a task easier, which, after taking the Differentiating Instruction course, Nicole no longer considered an effective example of differentiation (interview, 4/10/14). 
International student teaching. Nicole spent the second half of the fall 2013 semester student teaching in Belfast, Northern Ireland. The teachers in this school used an approach to instruction that they termed differentiation, but Nicole viewed this approach as very different from Tomlinson's model. She described how she "didn’t really like how it was done over there. And so I think that seeing that sort of helped crystalize a lot of things, where I was like, OK I know it's not supposed to look like that" (interview, 1/27/14). Thus, before Nicole studied differentiation in depth through the Differentiating Instruction course, she spent time in a system in which teachers responded to variance in student needs, particularly readiness, using a significantly different approach.

According to Nicole, the principles of teaching up, respectful tasks, and a supportive learning environment were absent from the school in Belfast where she student taught. Nicole described how tasks were "watered down" for lower readiness students (reflection, 2/14/14) in that they would complete simplified versions of worksheets or would just answer fewer questions from the same set of questions other students were assigned. Nicole felt that this reflected teachers having lower expectations for students they perceived as having limited capabilities, and she characterized it as disrespectful teaching (reflection, 2/14/14; reflection 3/23/14). She described an example of this as occurring when:

they would do practice reading comprehension passages. They would all read the same passage. The lower students would have different questions that in my personal opinion were very basic. Really not asking for the same level of deep understanding. More just surface level, fact recall. (interview, 2/24/14)

According to Nicole, the teachers in the school frequently yelled at students and embarrassed them in front of their peers. She also viewed the practice of frequently 
announcing which students were in the lower readiness group as detracting from the learning environment in this context (interview, 2/24/14). Ultimately, Nicole felt that this experience did not play a significant role in her developing conceptions of differentiation, since she "figured out pretty quickly that it was not...differentiation" and then "tossed out" the ideas from her thinking about the model (interview, 12/09/14).

Prior coursework. Nicole's first formal introduction to differentiated instruction was in an introductory curriculum and instruction course. This was the course in which Nicole received the most instruction about the model prior to spring 2014; one of Tomlinson's books was an assigned reading for the course (interview, 1/27/14). As noted above in the subsection on Misconceptions, Nicole brought away from this early course the understanding that differentiated instruction called for individualized instruction. She described her response to learning about the model for the first time as being "very overwhelmed" (interview, 1/27/14):

This is a day burned into my mind. The lesson...was about differentiation, and I remember all of us being like, How does this work? This feels so different from what school is supposed to look like! (interview, 1/27/14)

The instructor for this introductory course encouraged the students in Nicole's class to take the Differentiating Instruction course later in their teacher education programs. Nicole explained, "We talked about differentiation a little bit in that class, but there are so many things to talk about in that class, and so she encouraged us, if you get time somewhere in your time in Curry, definitely take it" (interview, 5/01/14). According to Nicole, differentiated instruction was a topic that received a limited amount of time and attention in this introductory course, as there were so many other topics to learn about 
(interview, 5/01/14). While differentiation was mentioned in some of Nicole's other courses, it did not receive substantial treatment in them (interview, 5/01/14).

Nicole also noted that the lesson plan template she learned to write lesson plans with during her coursework and then used to plan lessons for field placements had "a tiny box at the end asking for how we differentiated" (reflection, 4/06/14). Nicole continued, "I expected, therefore, that this box could be filled with a couple of bullet points so I could 'check the box' that I had differentiated that day" (reflection, 4/06/14).

Reactions to teacher education program factors. Nicole had strong views on the relationship between her experiences in her teacher education program and the conceptions of differentiated instruction she developed, which she shared during interviews in May 2014 and December 2014.

Reactions at course conclusion. In the spring, Nicole's thoughts about the relationship between her teacher education experiences and her conceptions of differentiation centered on her coursework, not on her experiences in field placements. At the beginning of the course, although Nicole characterized her teacher education coursework as "special" because "differentiation has been very much woven throughout all our classes," she also described it as a "couple years of education telling me vague things" about differentiation (interview, 1/27/14). Nicole felt that differentiated instruction had been referenced in positive terms and encouraged in other coursework, but that it had not been taught with enough detail and specificity.

At the conclusion of the course, Nicole expanded on her reactions to the treatment of differentiation in her prior coursework, echoing the comments she had made in January: 
I would say it's definitely something that comes up in every class, but...I think part of the reason that some of us feel so overwhelmed or confused by it is that it is, I think it's something you need to learn in the depth that we have this semester...so it's good that it's brought up, and some people are going to come to their understanding of it in different ways, but it was definitely one of things where I was like, I hear this word, and it's everywhere in educationspeakdifferentiation - but like I don't really get what it means. I kind of get what it means. I know that it sounds good in interviews. You know, that kind of thing. So yeah, I would say there was not a lot of depth to that conversation. In teacher preparation...we needed more on differentiation. (interview, 5/01/14)

In response to her belief that her previous teacher education coursework had not treated differentiation with enough "depth," Nicole concluded that an entire course on differentiating instruction should be a required component of a teacher education program (interview, 5/01/14). She believed that the reason many teachers do not differentiate "with fidelity" is because they do not have the knowledge and skills to do so (interview, 5/01/14). She explained:

I really do think that, one, I have more to learn about differentiation, and two, it's taken me the semester [slows pace of speech and emphasizes each syllable in word "semester"] to get to the point that I'm at now, and I feel like I'm graduating in a better place than I would have if I didn't take the class, so I don't think I'd be able to go into a school and practice differentiation well just with the basic education we get as Curry students, so yes, I think everyone should have to take it. (interview, 5/01/14)

Nicole emphasized her conclusion that it took her an entire semester of study to develop deep enough conceptions of differentiated instruction to begin to implement it with fidelity. She believed that, without this depth of understanding, she would not have been able to differentiate well in her future practice.

Nicole also noted that she not seen differentiation modeled by her university instructors in courses other than the Differentiating Instruction course (interview, 4/10/14). She tied this realization to the larger issue of the frustration she occasionally experienced during her teacher education coursework when she perceived there to be a 
"disconnect between what we know and are being taught are best teaching practices, and the way that we are taught" (interview, 4/10/14). Nicole characterized this as a "common complaint among students" in her program (interview, 4/10/14). Specifically, Nicole wished her instructors had modeled more hands-on learning, small group discussion, and communicating clear course learning goals, in addition to differentiating instruction (interview, 4/10/14).

Subsequent reactions. During an interview in December 2014, when Nicole was halfway through her first year of teaching, I asked her to reflect on the relationship between her conceptions of differentiated instruction and many factors involving her personal characteristics and teacher education experiences that she had mentioned in previous interviews and reflections (see Appendix I). Nicole considered each of these factors and classified them as enhancing the development of her conceptions of differentiation, detracting from her developing conceptions, or playing no role in her developing conceptions, or she indicated that she was uncertain as to whether it was related to her developing conceptions. After she had explained her responses to all factors, I asked Nicole to consider each factor she had characterized as playing a role in the development of her conceptions, either by enhancing or detracting from it, and to identify the three factors that had played the most significant roles. All three factors she selected were related to her teacher education program. In descending order of importance, they were her experiences in the Differentiating Instruction course, other coursework, and student teaching.

The factor with the strongest relationship to Nicole's conceptions of differentiated instruction was the Differentiating Instruction course. This relationship 
was one of enhancement and support for her developing conceptions of the model. In an interview the month before, Nicole had related that the most helpful knowledge she had gained from the course centered on the importance of clear learning goals. She had recently taught a math unit for which she had mapped out specific knowledge, understanding, and skills for the first time in the fall, and she believed that her teaching for the unit had been much better than any other unit she had taught. She described, "I knew what I wanted them to know, understand, and be able to do by the end of it. And so I was like, 'Oh, it is true! Knowing what I want from them helps my teaching!'” (interview, 11/14/14). In December, after selecting this factor as the most influential, Nicole explained that the course gave her the "mental model" of differentiation that she had brought into the classroom with her, one in which she understood it to be a philosophy as a set of strategies (interview, 12/09/14). Nicole described this mental model as giving her hooks or "places to hang all the little ideas about differentiation" (interview, 12/09/14). Nicole viewed the conceptual framework of knowledge and beliefs about differentiated instruction she formed during the course as the most important influence on her conceptions of the model.

According to Nicole, the factor with the second strongest relationship to her conceptions of differentiated instruction was the coursework she completed prior to the Differentiating Instruction course in which differentiation was either mentioned in vague terms or, in the case of her introductory curriculum and instruction course, left her with misconceptions. Nicole explained:

I still remember reading all the stuff about differentiation [in the introductory course], and we were all like, what? I'm actually going to say that that detracted from my understanding [of differentiation], because I still very clearly remember my whole class being like, that means we have to make individual plans for every 
single student, which is totally wrong, and I think that, like, that's dangerous, because I was in college, and I was already in a teacher prep program, and something in that class gave me that impression, and I'm glad that I went out of my way-you know the D.I. class with Dr. Tomlinson is not required, this one is required. So I'm glad that I went of my way to learn something else about it, but you know, had I not, I don't know what would have happened with my little mental model of differentiation....[The earlier course] made me feel scared of differentiation and reluctant, and I really had to seek it out on my own to overcome that, and that bothers me just thinking about everybody else that graduated with me or before me or after me [who did not take the Differentiating Instruction course]. (interview, 12/09/14)

As Nicole noted, the Differentiating Instruction course was an elective for preservice teachers, which most students in the B/MT and PG/MT program did not take. Nicole believed that the other students in her program who did not take this elective and whose primary exposure to differentiation was in the introductory course she discussed would not be prepared to differentiate effectively. Nicole's comments suggest that taking the Differentiating Instruction course influenced her knowledge and beliefs about differentiation by undoing her reluctance and fear about implementing it and changing her conceptual framework or "mental model" of its nature and function.

Last, Nicole identified her experience student teaching as having the third strongest relationship to her conceptions of differentiated instruction in that it enhanced her understanding of some aspects of the model. Nicole described her CI as "the master of community and routines" in terms of her "ability to create the classroom culture very well that lays the groundwork for everything she does throughout the year" (interview, 12/09/14). Nicole characterized her CI as "a phenomenal influence" on Nicole's practice and believed she carried the knowledge and skills of establishing a positive learning environment she gained during student teaching into her first year in the classroom (interview, 12/09/14). "The learning environment is super important to me in terms of 
being able to differentiate later," she noted (interview, 12/09/14), explaining why this aspect of her student teaching experience supported the conceptions of differentiation she developed during the course.

1(b) Summary. As Nicole made deeper meaning of the philosophy and practice of differentiated instruction throughout the spring semester, she identified previous experiences that played a role in her developing knowledge and beliefs about the model. These factors involved her personal background and her teacher education program.

The personal background factors Nicole identified involved her prior beliefs about teaching and learning that she brought to the course, her own K-12 educational experiences, and other relevant personal characteristics. Nicole found that the Differentiating Instruction course prompted her to define her existing beliefs about teaching more precisely and then gave her the verbiage to discuss them. She described experiencing a fluid process during the course in which she folded her new beliefs about teaching related to differentiation into her existing beliefs. Nicole could not recall experiencing differentiation as a K-12 student and felt that those experiences did not support her developing conceptions of differentiation, although one sixth grade teacher's practice did provide her examples of building teacher student connections in a supportive learning environment and of presenting content through multiple modes of instruction. In terms of personal characteristics with a connection to her developing conceptions of differentiation, Nicole viewed the need for flexibility in response to the "messiness" of teaching that could not be planned for as difficult for someone like Nicole who instinctively preferred high levels of organization. Recognizing that she would not achieve perfection in differentiation as a first year teacher appeared to cause Nicole 
concern. This seemed to be connected to her mindset, which she described as not fully orientated toward growth. While she believed her current mindset status did not support her conceptions of differentiation, she also viewed her experiences in the Differentiating Instruction course through which she was prompted to consider the importance of mindset in the classroom as supporting her developing conceptions of the model. She ultimately concluded that having been educated in a fixed mindset educational system featuring tracking and prevalent competition among students detracted from the development of her conceptions of differentiated instruction, since it influenced beliefs about teaching and learning that she would have to shift to differentiate effectively.

Nicole noted significant relationships between her evolving conceptions of differentiated instruction and her previous experiences in her teacher education program, including her coursework and student teaching. Some of these experiences supported her developing conceptions, while others detracted from it. Nicole felt that her experiences in third and fourth year field placements and student teaching abroad did not play a significant role in her developing conceptions of differentiation, since she did not see much differentiation in her early placements and dismissed the model of "differentiation" she saw in Belfast as totally misaligned with her conceptions of the approach. Her student teaching experience appeared to influence her understanding of establishing a supportive learning environment and managing classroom details flexibly, but did not involve the elements of high quality curriculum, ongoing assessment, and modified instruction based on variance in student needs.

Ultimately, out of all the factors Nicole mentioned in reflections and interviews that had a relationship to her developing conceptions of differentiation, she identified 
three that played the most important roles. All three were related to her teacher education program: the Differentiating Instruction course, other coursework, and student teaching. Nicole viewed the conceptual framework of knowledge and beliefs about differentiated instruction she formed during the course as the most important influence on her conceptions of the model. The factor with the second strongest relationship to her conceptions of differentiated instruction was the coursework she completed prior to the Differentiating Instruction course in which differentiation was mentioned in vague terms or she was left with misconceptions. Nicole identified her experience student teaching as the third strongest factor in that it enhanced her understanding of some aspects of differentiation that were modeled by her CI.

\section{Nicole's Phase 2 Experiences}

In March 2014, Nicole was hired by Oakhurst County Public Schools for the 2014-2015 academic year. Oakhurst County is one of the largest school districts in the mid-Atlantic region and was the same district Nicole had attended as a K-12 student. In 2014-2015, approximately $40 \%$ of its students are white, $25 \%$ are Hispanic, $20 \%$ are Asian-American, and 10\% are African-American (Oakhurst website, 2015). Around 25\% of its students receive free or reduced price meals, 15\% are English language learners, and $15 \%$ receive special education services (Oakhurst website, 2015). The average cost per student is over $\$ 13,000$, and around $90 \%$ of its students graduate on time (Oakhurst website, 2015).

When Nicole was hired in March, she was informed by Oakhurst that she would teach fifth and sixth grade science and math at Williams Elementary School. Williams is located in a wealthy area and is known for its many enrichment activities for students 
(Nicole, interview, 4/10/14). In 2013-2014, the most recent year for which information was available, Williams served about 600 students, around one-third of whom received free or reduced price meals (Oakhurst website, 2015). In fifth grade, approximately 80\% of students passed the end of grade tests in reading, and approximately $75 \%$ of students passed in math (Oakhurst website, 2015). In sixth grade, approximately $90 \%$ of students passed in reading, and 95\% passed in math (Oakhurst website, 2015). Thus, Nicole completed the second half of the Differentiating Instruction course believing that she would be teaching in the fall at a school with academically successful students and with only around one-third of students from low socio-economic backgrounds.

In May, just as the Differentiating Instruction course concluded, Oakhurst informed Nicole that she would not be teaching at Williams. In late June, Nicole was told that she would instead be teaching third grade at Lambert Elementary School. Lambert serves around 900 students, with around 55\% receiving free or reduced price meals and just below 50\% identified as limited English proficient (Oakhurst website, 2015). Just under $50 \%$ of students are Hispanic, around $30 \%$ are white, $10 \%$ are Asian, and $10 \%$ are African-American (Oakhurst website, 2015). In 2013-2014, the most recent year for which information was available, approximately $70 \%$ of fifth grade students passed the end of grade tests in reading, and just under $50 \%$ of students passed in math (Oakhurst website, 2015). In sixth grade, approximately 60\% passed in reading, and 75\% passed in math (Oakhurst website, 2015). In third grade, to which Nicole was assigned, approximately 55\% passed in reading, and 45\% passed in math (Oakhurst website, 2015). In sum, Lambert is a school in which more than half of the students come from low socio-economic backgrounds, just under half are limited English proficient, more than 
two-thirds are students of color, and only around half of its third graders passed recent end of year tests.

Nicole is one of seven third grade teachers. She began the year with 20 students but gained an additional student in October, bringing the total to 21. Eleven are female, and 10 are male. Seventeen students are Hispanic, including nine girls and eight boys. One girl and one boy are Caucasian. One girl is African-American, and one boy is east Asian. At the beginning of the school year, the students were eight or nine years old.

\section{Research Question 2(a): What were Nicole's conceptions of the model as she first} entered the classroom, and how did her conceptions change during the fall, if at all?

The first interview I conducted with Nicole in the fall occurred a week before school started. She reported that she had not thought about differentiation much over the summer:

It was hard to do a lot of thinking... about all these things in such an abstract way when there are no kids to assign them to, so I think I spent my summer thinking in big, fuzzy ideas. And yes differentiation was in there, but so was, how am I going to set up my classroom? What do I need to buy?.... So I feel like it was there, but it definitely wasn't something I was concretely thinking about until I got into the orientation process and really started to look at the curriculum and started to think about, what is this going to look like in my classroom? (interview, 8/25/14)

I spoke to Nicole three days after she had received her class list, which prompted her to think about what differentiation would "look like" in her classroom in much more detail.

Nicole had learned that most of her students would be English language learners (interview, 8/25/15). She had also recently completed orientation training on working with learners living in poverty in preparation for the "very needy" students she would teach, in addition to some students from "solidly middle class" backgrounds (interview, $8 / 25 / 15)$. She described the student body as "extremely diverse," saying that learning all 
of this information about her future students "definitely made me kind of anxious, but I also said, okay, I took differentiation! So hopefully I can use it, 'cause I'm gonna need it!” (interview, 8/25/15).

Differentiation was a topic addressed in Oakhurst's new teacher orientation session that Nicole attended in August just before our interview. She described her reaction to hearing differentiation discussed during the session:

I feel like differentiation is such an education buzzword, and it's definitely something the district people were using all the time in orientation last week. And of course I'm sitting there being like, I wonder if they're meaning differentiation the way I'm meaning differentiation, because that was such a big subject in class of like, people say differentiation and don't really use it properly, and I think that may be where some of my anxiety is coming from. I don't want to be the obnoxious new teacher in the room who's like, "Well differentiation actually means this, and I took a class on it!" But I am excited, and I feel like I put myself in a really good position by taking the course because it is the reality of teaching today. To teach well, you have to be able to differentiate well. So I feel like I put myself in the best position possible by taking the class and learning about it, and that doesn't mean I'm going to always do it well, but at least I have it in the back of my mind and can hopefully draw on it and use it, 'cause now I definitely know I'll need to. (interview, 8/25/14)

It seemed that Nicole's experiences during the orientation sessions caused her to wonder whether, in the context of her new school in this particular district, differentiation would be defined by Tomlinson's model in the same way Nicole had learned about it as a preservice teacher. In light of the diversity reflected in her classroom, Nicole began the school year believing there would be a significant need for differentiation in her classroom and that she had placed herself in the "best position possible" by learning about the instructional approach in depth in her teacher education program.

Four weeks into the school year, the students in Nicole's classroom had moved from abstract entities into learners whose needs she knew well and recognized the differences among. She explained that her conceptualization of differentiation had not 
changed, but that she understood issues surrounding its implementation, or lack of implementation, more clearly:

I don't think [my understanding has] changed, but...I know that I'm not doing it as well as I ideally would want to, and that's been frustrating. I feel like I'm not, you know we talked about differentiation in the spring as this moral obligation thing, and I still believe that, but it's like, I haven't gotten that off the ground and like, oh my gosh, and I look at the class, and the need is there. I don't think there's a class where the need isn't there ever in the history of the world, but now that I know them, the need is so there. They're so so different and they have such different needs, that it's like, this is something I need to be doing... I'm trying to give myself the forgiveness of like, I couldn't come in on day one and be like, Here's a learning menu or a Sternberg activity! Differentiation takes time to get off the ground like anything else, but it is a little bit like, I'm empathizing more with the teachers we read about in [the Differentiation Course readings] that are like, I don't have time! Or I don't know how this would work in my classroom! 'Cause I'm like, okay, I hear ya! So I don't think it's changed in terms of how I think about it and how I want to implement it, but the reality of implementation is now scarier and more challenging that I think I gave it credit for. (interview, 9/30/14)

Nicole's comments demonstrate the interconnectedness of her conceptions of differentiated instruction and her related practice during the fall. While she reported that "how she thought about" differentiation and "how she wants to implement it" had not changed, she also reported that thinking about differentiation in a classroom context with her own students caused her to recognize in a new way that the "need is so there." In this way, her belief in the importance of differentiation seemed to be heightened or underscored. Her beliefs about the "reality" of implementation also appeared to change once she was in the classroom. She became more understanding of teachers she had heard about or seen as a preservice teacher who did not differentiate because they felt they have no time for it, and she characterized it as a more challenging endeavor than she had anticipated when learning about it in a teacher education context.

Nicole's Phase 2 concept map. In October, once Nicole had been teaching for 
around two months, she repeated the concept map activity she had completed during our final Phase 1 interview in May. I asked her to create a representation of the relationships among the five elements of a differentiated classroom (see Figure 4) and to think aloud as she created it. In Phase 1, Nicole had created a concept map in which learning environment and leading students and managing routines formed a box encompassing the ongoing cycle of curriculum, assessment, and instruction (see Figure 3). She had characterized learning environment as slightly more important than the other four elements, using a building metaphor to describe learning environment as the first layer of foundation and leading students and managing routines as the second foundational layer (interview, 5/01/14). Within the cycle of curriculum, assessment, and instruction, she described curriculum as the most important starting point (interview, 5/01/14).

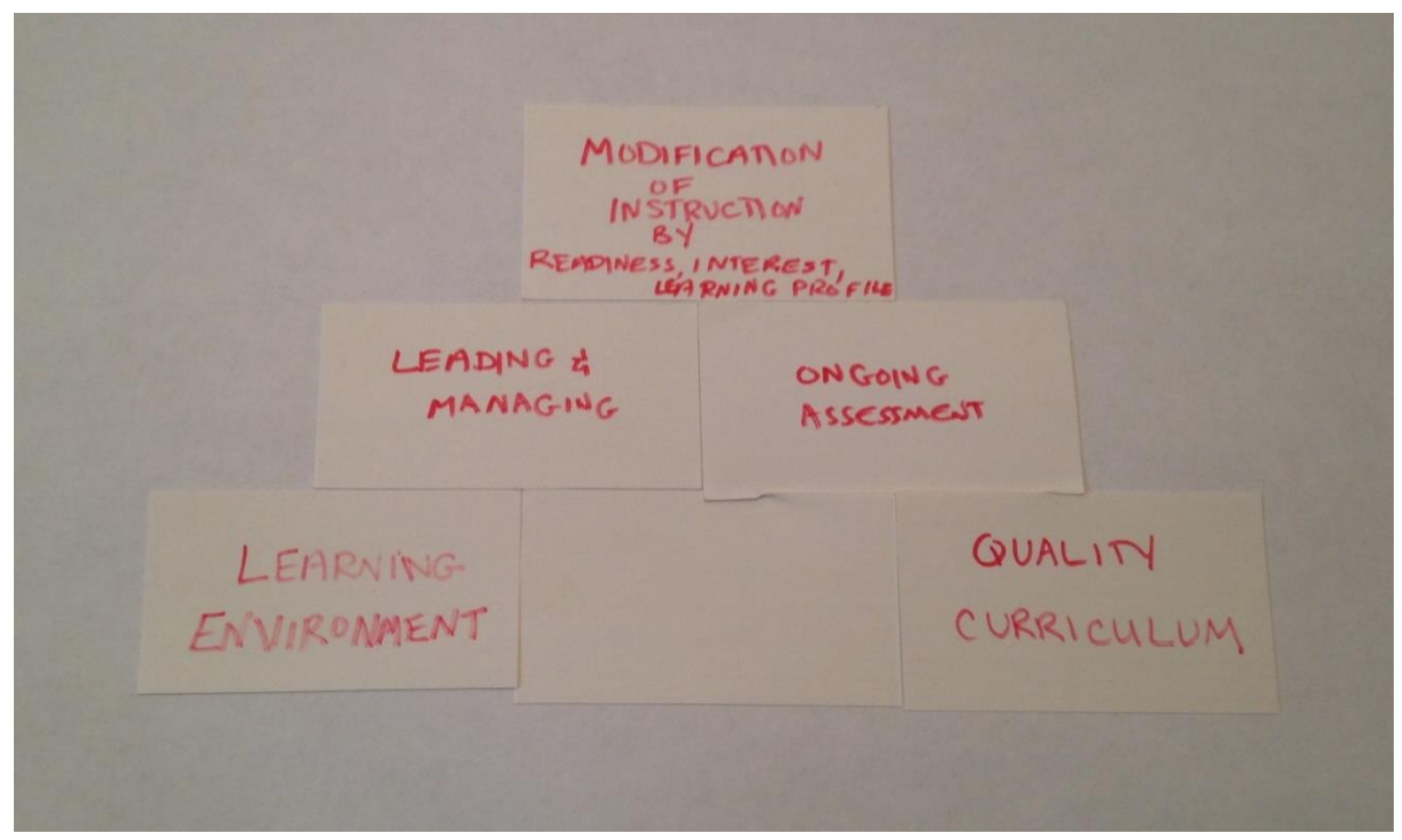

Figure 4. Nicole's second concept map illustrating relationships among the five elements of a differentiated classroom. Created during Phase 2 Interview 2 on October 20, 2014. 
Nicole's Phase 2 concept map was configured differently. She created a pyramid shape with the five elements, putting learning environment and quality curriculum at the base. Leading and managing and ongoing assessment formed the middle row of the pyramid, with leading and managing located on top of learning environment and assessment located on top of curriculum. Modification of instruction sat at the pyramid's top. In this tiered or layered approach, Nicole described, the elements "build on one another" (interview, 10/20/14). She explained the base and middle layers of the pyramid by saying that learning environment and curriculum are the foundation or "bedrock" of the classroom, the ability to lead and manage comes from the right culture and climate in the classroom, and assessment allows you to determine the quality of the curriculum you present. Last, she said she placed modification on top since "it's almost the smallest part, because all these other things are so much bigger and have to happen first...It's the last piece that you can't put on until you have everything else" (interview, 10/20/14).

After she finished her explanation, she indicated she was aware that this configuration was different from the one she had created in May, saying she thought her Phase 1 map had been more of a circle (interview, 10/20/14). I then showed Nicole her Phase 1 map (see Figure 3) and asked her why she thought the new one was structured differently. She explained:

This [configuration] right now as an actual teacher is easier for me to conceptualize because I feel like I've been viewing my practice since the beginning of the school year, like I feel like the whole year is building on everything else, and that's like the foundation you build from day 1 impacts your management all year long....I feel like that concept of constantly having to build on what you've already done has become how I'm thinking about teaching....I think in May it was just hard to understand what the day to day is actually like, and so I feel like I do modification of instruction not as often as I maybe would have thought. (interview, 10/20/14) 
The differences between the two versions of Nicole's concept map seemed to reflect changes in how Nicole conceptualized the practice of teaching, rather than specific changes in her views of the relationships among the elements of a differentiated classroom. At this point in her practice, Nicole saw teaching as a process of building layer by layer, with this layering process applying to everything from the establishment of a positive learning environment and classroom management practices to teaching students foundational content in language arts or math and building upon it. Conceptualizing her teaching practice this way seemed to cause Nicole to reconceptualize the elements of differentiation this way as well.

The new configuration also appeared to reflect changes in how Nicole thought about the modification of instruction. She now saw this element of differentiation as the "smallest" element, with the other elements being "much bigger," perhaps reflecting the infrequency of modification of instruction's presence in her teaching practice to date. In the fall, she had come to view all four of the other elements as necessarily foundational to modification of instruction, believing that it could not be accomplished effectively without the other elements solidly in place.

2(a) Summary. In light of the diversity reflected in her classroom, Nicole began the school year believing there would be a significant need for differentiation in her classroom and that she had placed herself in the best position possible by learning about the instructional approach in depth in her teacher education program. Although Nicole said that her knowledge and beliefs about differentiation had not changed since the spring, she also reported that thinking about differentiation in a classroom context with her own students caused her to appreciate the need for it in a new way. Thus, her belief 
in the importance of differentiation seemed to be heightened. When considering the relationships among the five elements of a differentiated classroom in the fall, Nicole came to see modification of instruction as a smaller component resting upon the other four elements. This seemed to reflect her infrequent modification of instruction in her teaching practice to date and the need for the other four elements to be strongly established as foundational to effective differentiation.

\section{Research Question 2(b): What was the relationship between Nicole's conceptions of the model and her teaching practice?}

- "I came in as prepared as I ever could have been, but...there's no comparison to actually doing it" (interview, 9/30/14).

- "So much of teaching is about balancing the needs of what you have and the needs of, this is what has to get done, and you have to live in that tension" (interview, 9/30/14).

- "It's my first year of teaching, and I have to have realistic goals about what can happen in a year (interview, 10/20/14).

- "Differentiation and survival mode are not super compatible" (interview, 12/09/14).

These statements, which Nicole made throughout the fall of her first year in the classroom, were typical of her comments about the relationship between her knowledge and beliefs about differentiation and her teaching practice. Nicole implemented many practices reflecting key principles related to all of the non-negotiable elements of a differentiated classroom, although she felt the demands of the first year posed obstacles 
that prevented her from implementing the model as she had anticipated as a preservice teacher.

As I describe in greater detail in the Administration and Mentoring section below, I interviewed Nicole's mentor, Sharon, during the fall. After observing and co-teaching with Nicole several times at the beginning of the year, Sharon described Nicole's approach to teaching:

I think that she's thinking all the time about how she can meet the needs of her students. That's so important. She's coming in with the lens that all kids are not going to be put into one group. That's going to help. It can be overwhelming at times, but also a help because if you learn that right from the beginning, it just sets you up for a lot more successful teaching. So she's coming in with that lens of looking at kids. (interview, 10/20/14)

Sharon observed that Nicole began the year by "looking at kids" or studying her students to identify their varied needs and to determine how to meet them. This approach is based on the recognition that all students will not have the same needs and cannot always be treated as one group to learn well. While Sharon acknowledged that having this approach to instruction can be overwhelming for first year teachers, she also believed it would position a teacher for successful teaching in the long run.

At the end of September, Nicole reported that she was frustrated because she was not "doing" differentiation as "well" as she wanted to, saying that she had not gotten differentiation "off the ground" yet (interview, 9/30/14). In October, she described differentiation as occurring a bit more in her practice, citing her new use of unit preassessment data to inform her instruction in social studies as an example, but saying it was still not "fully-fledged" (interview, 10/20/14). During our last Phase 2 interview in December, Nicole described differentiation as "still...not happening as much as I would want it to," and she characterized this as disappointing to her (interview, 12/09/14). 
At the start of the school year, the classroom element to which Nicole devoted the most attention was the learning environment.

Learning environment. During the first month of school, Nicole focused heavily on building a welcoming community within her classroom. It was her primary focus during the first week of school, which her administration encouraged (interview, 9/30/14). Beginning the second week of school, however, teachers at Lambert were expected to cover substantive content, including math content that Nicole's students found challenging. She expressed disappointment that she did not have more time to focus primarily on community building before moving into a heavy focus on curriculum coverage (interview, 9/30/14).

Nicole felt that her efforts to create a supportive community in her room were somewhat challenged by the structure of Lambert's language immersion program. Lambert offered the option of French immersion. Students enrolled in this program took math and science in French and their other classes in English. Half of Nicole's students were enrolled in the French immersion program. These students went to their French classroom immediately upon arriving at school in the morning and did not join Nicole's class until the middle of the day. Thus, for the first half of the school day, Nicole only saw half of her class. These students, who were not enrolled in the immersion program, were her students with the most limited English proficiency, with the exception of one student who transferred out of the immersion program several months into the year (interviews, 9/30/14,10/20/14). Nicole did not develop personal connections with some of the students in the French immersion program to the same extent as the students she had all day (interview, 12/09/14). She explained: 
If they're naturally quiet and they're not as needy of a student, I don't have as many interactions with them...I spend literally half as much time with them...so there's definitely a piece missing from my ability to reach... some of the French kids, simply because I don't know them as well. (interview, 12/09/14)

While the structure of the immersion program posed obstacles in terms of Nicole's developing personal connections with some of her students, it did provide an opportunity for Nicole to build a special community with the students she had in the morning.

She observed that this group, which included her less proficient English speakers, was much more confident in the morning than later in the day when their peers from French immersion joined the class. These students participated and volunteered answers far more frequently in the morning than they did in the afternoon, when the students from French immersion were "jumping out of their seats to participate" (interview, 9/30/14). Nicole suspected that, because most of her class had known each other since kindergarten, the students' ideas about "who's good at school, who's bad at school” were “engrained" by third grade (interview, 9/30/14). She viewed it as her job to help her morning group overcome these ideas and become comfortable with participating in front of the other students (interview, 9/30/14). One way in which Nicole worked to accomplish this was to position students in the math and science group in leadership roles. She explained:

I would teach the morning group a game and then we would do it again in the afternoon, which gave them the opportunity to already have the language for it, and it was like, oh, you guys have done this, and that makes them kind of the experts. (interviews, 9/30/14)

Casting her morning group students as experts in her classroom encouraged their confidence, as did Nicole's use of other strategies such as having students share their ideas with a partner or small group before sharing them in front of the whole class 
(interview, 9/30/14).

During each of my observations, I watched Nicole engage in a number of practices and interactions that contributed to the kind of supportive learning environment called for by a differentiated approach to instruction. For example, she built community among her students by having them draw figures of themselves reflecting their varied learning preferences (Tomlinson \& Imbeau, 2010, pp. 153-154) to share with the class (interview, 9/30/14; observation, 9/30/14), had students eat with assigned lunch buddies to learn something new about them to share with the rest of the class (observation, 10/20/14), and asked a Nepalese student who had recently moved to the United States and had studied Mandarin to show his classmates how to write words in Chinese characters so they could see the unique skills and experiences he brought to the class (observation, 11/14/14). She targeted her interactions with students to form personal connections with them by asking questions about their lives outside of school (observations, 11/14/14, 12/09/14) and hugging each one when they left school for the day (observation, 12/09/14). And she communicated to students that she expected them to do their best, and that they would grow if they tried, by making comments to students as a group and individually such as, "I know this is difficult. I'm asking you to think. But it's important for you to try" (observation, 12/09/14).

Classroom management. In addition to creating a supportive learning environment, Nicole was heavily focused on establishing routines during the first week of school (interview, 9/30/14). She believed that devoting significant time to laying this "groundwork" was necessary for the classroom to run smoothly (interview, 8/25/14). By the fourth week of school, during my first observation, the students in Nicole's class 
executed a large number of routines without prompting beyond one cue and generally without correction. Examples of the smoothly executed routines and classroom procedures I observed included students:

- Stopping discussions with a partner immediately when Nicole rang a bell $(9 / 30 / 14)$.

- Lining up single file in the classroom and walking down the hall in silence $(9 / 30 / 14)$.

- Moving from one location in the room to another, individually or in groups, in silence $(9 / 30 / 14,11 / 14 / 14,12 / 09 / 14)$.

- Increasing the speed at which they moved from one location in the room to another when Nicole counted backwards from 10 (9/30/14).

- Speaking in significantly softer voices when asked to use a "level one" rather than a "level two" voice (9/30/14).

- Engaging in choral response to repeat key directions back to Nicole (10/20/14, 11/14/14).

- Self-assessing on a scale of one to three the degree to which they had remained on-task during an independent activity (9/30/14).

For students to execute these routines with the immediacy and efficiency I observed, substantial prior practice was necessary. On the rare occasions students did not complete the classroom procedure according to the established expectations, Nicole asked them to repeat the procedure. In a typical example, during one observation, I recorded the following occurrence in my field notes in which Nicole asked the class to move from their desks to the front of the classroom for a lesson: 
Nicole tells the students to go and sit on the carpet. Three students walk very quickly, almost jogging, to the carpet. Nicole looks at these students and then says, "Everyone go back to your seats - that's not the way we move in our classroom." All of the students immediately return to their seats and sit down. Nicole says, "Now we're going to try again. Everyone walk to the carpet." $(10 / 20 / 14)$

All students walked to the carpet after this re-direction. Using this strategy of repetition, Nicole reinforced the expectation that procedures would be followed in her classroom and gave students the opportunity to practice completing the procedure correctly.

These well-established routines allowed students in Nicole's classroom to complete independent and small group work without teacher involvement. In particular, she reported spending a significant amount of time teaching students how to work successfully within the Daily 5 literacy instructional framework and the Daily 3 math instructional framework used in Oakhurst (interview, 8/25/14). This approach to management and small group instruction called for students to transition among different types of work in different locations based on one simple cue and to self-assess the degree to which they had been on-task the whole time (observations, 9/30/14, 12/09/14). The students' successful execution of the classroom management structures involved in Daily 5 and Daily 3 gave Nicole the freedom to work with one student or one group as needed.

High quality curriculum. As Nicole reflected on the nature of her third grade curriculum during the fall, the challenges she faced in its design and execution involved three issues: expectations surrounding Lambert's curriculum map, the role of clear learning goals, and supporting students who were significantly below grade level.

Curriculum map. Nicole's curriculum was based on the third grade curriculum map, a detailed document created at the school level based on state standards for third grade and Oakhurst's third grade pacing guide. The curriculum map provided a day-by- 
day guide for covering content each quarter (interview, 8/25/14). Before school began, Nicole viewed the curriculum map to be of high quality and a useful tool to "measure" herself to see if she was "on track," although she did express some initial concerns that she might be "penalized" somehow for doing something differently than was outlined in the document (interview, 8/25/14).

At the end of September, Nicole described how she had begun covering substantial content at the beginning of the second week of school, including a very challenging unit on place value in math (interview, 9/30/14). Although she believed her class was not yet ready for this abstract content and needed more time to practice the routines of Daily 3 math and Daily 5 literacy, Nicole moved forward because of the school curriculum map. Several weeks into the school year, a number of the third grade teachers had fallen behind the pace indicated in the math curriculum map, since students lacked background knowledge about the topic and struggled with conceptual understanding. When the teachers raised this issue during a meeting with the math instructional coach for their grade level, the team gave themselves "permission to not follow it lock-step," at which point Nicole felt she had more flexibility in responding to her students' need for a slower pace (interview, 9/30/14). Toward the end of the first quarter, however, that "permission was taken away" when the third grade teachers "got behind" with the math curriculum (interview, 12/09/14). Nicole explained:

I mean it's not like explicitly like, oh, the permission was taken away, but yes it was. It's one beef that the team had was, yes...we're giving you permission not to stress about the curriculum map, la, la, la, and then at the end of the first quarter, we were told, we need to shave a week off of fractions, and we need to shave a week off somewhere else because we need to catch up, and that was a big frustration for the team, because it was like, you told us that it was okay to go slow. (interview, 12/09/14) 
As a result, for the majority of the fall, Nicole felt that she did not have permission to slow down her pace of covering the curriculum when she believed her students would have benefited from that change. The power to make decisions about the level of fidelity to the curriculum map that was expected from the teachers appeared to rest beyond Nicole and her colleagues with the instructional coach for her grade and other administrators.

For Nicole, this lack of control over her curriculum delivery pace created discontinuity between her conception of differentiation and her teaching practice. She explained:

This is just like where all of the pressure to just do and just cover and just move on comes from...I'm thinking about... when we were in place value, and my kids... have a lot of general gaps in their number sense... and things were just very hard for them, and I remember feeling like I wanted to stay in place value forever. I was like, I could teach place value all year long. And it was very hard to find the line between, and part of this is inexperience, finding the line between how do I meet their needs and meet them where they're at, and do all these things I want to do with differentiation and also not stay on place value for forever? So I think meeting the reality of there is curriculum, there are standards, there are expectations, was a challenge to implementation. And that doesn't mean it's impossible. It just means I as a first year teacher had a hard time with it....[W]ith defined starts and ends or defined "you should spend this long on it," I didn't feel it allowed me to do what was best for them [the students]. (interview, 12/09/14)

Soon after beginning her math unit on place value, Nicole realized that her students would benefit from spending more time on the content, while the curriculum map developed by her school prevented her from doing so. This situation seemed to be an example of Nicole's recognition as a first year teacher that teaching involves coping with the "tension" produced by balancing the needs of students and the needs of "what has to get done" (interview, 9/30/14). In this case, what needed to get done outweighed the 
needs of most of the students in Nicole's class, and she saw this as an example of how her standardized curriculum inhibited her implementation of differentiation.

Learning goals. As noted above in the section on her reactions to teacher education program factors subsequent to graduating, Nicole created clear learning goals reflecting what she wanted students to know, understand, and be able to do for the first time for one math unit in early November. While she recognized its importance to high quality curriculum both in the spring and in the fall, it was not something she did routinely when planning for lessons in the fall.

I observed Nicole teach a total of five lessons in language arts, science, and social studies across four classroom visits. In none of these lessons did I observe her present curriculum that focused on conceptual understanding or that made clear a big idea to students. Instead, the lessons I observed were designed to have students practice discrete skills in language arts such as spelling, reading fluency, and comprehension (observation, $9 / 30 / 14,11 / 14 / 14,12 / 09 / 14)$ and to memorize facts about animal classifications based on diet and facts about ancient China in social studies (observation, 10/20/14).

Students below grade level. Last, Nicole struggled to determine what to teach her students who were significantly below grade level, especially in reading. Nicole had at least seven students who were working on decoding skills at a kindergarten or first grade level (interviews, 9/30/14,10/20/14), while the third grade standards called for Nicole to teach more advanced reading skills, such as identifying and sequencing events in a complex text.

In October, Nicole wondered if she should focus her small group instruction with these students almost entirely on shoring up their decoding skills, rather than working on 
the skills targeted by grade-level standards (interview, 10/20/14). She was unsure whether to "take the standard down" or to "just focus on intensive guided reading because we need to bring...up" their reading levels (interview, 10/20/14). For example, in one lesson on identifying and sequencing events in a complex text, Nicole worked with a group of these lower readiness students using a first grade text (interview, 10/20/14). Rather than ask the students to practice decoding, Nicole read the text with the students and gave them cards with the events in the book written on them. She then asked the students to sequence the events (interview, 10/20/14). In this way, she had decided to "take the standard down" for this group, although she was unsure whether she had made the right decision.

By mid-November, Nicole had discussed this issue with the third grade team and concluded that she would find the way to have students significantly below grade level practice skills from third grade standards. She explained that her thinking about this issue had changed since our last interview:

I think the two aren't as mutually exclusive as I was thinking of them in that moment. Because it is still beneficial for them to be reading texts on their [lower] level, but it's important for them to be thinking about things like that, like what order do these things happen in? Can I retell in order the story? I mean that's going to be beneficial for them, regardless of whether it's a first grade standard or second grade standard or third grade standard. (interview, 11/14/14)

Nicole gave an additional example of what it might look like for her students below grade level in reading to work with a third grade standard by describing how, in her next unit on nonfiction, those students would work with her in guided reading lessons with appropriately leveled books to examine nonfiction text features, just as all of the other students reading on grade level would.

Ongoing assessment. Before the school year began, Nicole predicted that, of the 
five elements of a differentiated classroom, assessment would be her primary focus during the first few weeks of school. She explained:

I want to try and have the clearest picture possible of my students, and a lot of that is just getting to know them also, but I definitely want to have the best sense I possibly can as early as possible of where they're at, because I can't realistically differentiate to meet their needs if I don't know what their needs are, so that's definitely the piece that I'm thinking the most about right now. (interview, $8 / 25 / 14)$

This prediction had a clear relationship to Nicole's initial purpose in taking the

Differentiating Instruction course, which she described in January as developing the skill of recognizing the individual learner needs present in her class as quickly as possible so that she could respond to them efficiently (interview, 1/27/14). However, in the end, learning environment and managing the details of the classroom were the elements of differentiation on which she spent the most focus during the first weeks of school.

Throughout the fall, assessment was not a topic that Nicole discussed frequently during our interviews. She did mention that she used results from the Developmental Reading Assessment to determine student readiness for language arts and to form her groups for Daily 5 (interview, 9/30/14). In math, she used formal assessments like tests but also observed and took notes as individual students worked on problems or had oneon-one conferences with students to probe their thinking and look for misconceptions (interview, 9/30/14).

A related topic that Nicole did discuss at length during our October and November interviews was assigning report card grades. All elementary schools in the Oakhurst District used the same report card, which the district described as standardsbased in that it listed broad proficiencies reflected in multiple third grade standards and called for a grade to be assigned for each of these categories of knowledge, 
understanding, or skill (interview, 10/20/14, 11/14/14). The report card was not explained to Nicole until soon before the end of the first quarter, at which point she became concerned she would not have enough graded assessments to justify assigning a grade for each proficiency (interview, 10/20/14). As a result, Nicole changed her use of formative assessments and began giving students quizzes she did not feel were instructionally necessary so that she could have a graded assessment for every standard (interview, 10/20/14; observation, 10/20/14). Nicole was disappointed that she had not been instructed about the report card, specifically about the overarching proficiencies on which she would be grading her students, at the beginning of the school year so she could align her instruction accordingly (interview, 11/14/14). Ultimately, however, Nicole viewed the nature of Oakhurst's report card as supporting her implementation of differentiation, since she viewed it as offering flexibility in allowing her to reflect, through different kinds of grades, the progress students made with each proficiency during the quarter, as well as where their performance was relative to the grade-level standard (interview, 12/09/14).

Modifying instruction. Modifying instruction based on student readiness was the norm in Nicole's language arts and math instruction. The Daily 5 and Daily 3 instructional framework used throughout Lambert provided Nicole with a structure through which she grouped students for independent and small group work differentiated by readiness. I was unable to observe Nicole teach math, but in every language arts lesson I observed, students transitioned through Daily 5 tasks including independent reading, reading aloud to a partner, independent writing, and guided reading with Nicole (observations, 9/30/14, 11/14/14, 12/09/14). For this class, students were grouped for the 
first five weeks based on their DRA scores from spring of second grade, and then were based on their DRA scores from fall of third grade through December (11/14/14). Thus, once groups were established based on standardized assessment data, they were not shifted based on other formative assessment results.

At one point during the fall, Nicole hoped to modify her instruction by student interest during a social studies unit on ancient Egypt. As a preassessment for the unit, Nicole asked students to complete a portion of a K-W-L chart indicating what they already knew about the topic and what they hoped to learn. She noticed in light of what students said they hoped to learn that they had varied, specific interests, and she hoped to respond to those interests through her instruction during the unit, perhaps by having students read different trade books on their topic and share their findings with the class (interview, 10/20/14). However, Nicole did not end up modifying instruction during this unit. She explained that students were allowed to choose a particular contribution of the ancient Egyptians to draw a picture of for the classroom bulletin board, commenting, "so that was my one like, all right, there's some interest-based differentiation going on here" (interview, 11/14/14).

During our last interview in December, however, Nicole said that she had not modified instruction based on interest or learning profile in significant ways during the fall. She concluded:

I think that the readiness-based differentiation is pretty solid, because of the framework that allows me to pull reading groups and allows me to pull math groups and that focuses so much on, especially with math, preassessment, assessing along the way, changing the groups as necessary. But I think all the other types of differentiation are just still really not in place, and that depresses me if I think about it too much. So that's a goal. It's a goal for after break. (interview, 12/09/14) 
Nicole attributed the prevalence of modification of instruction by readiness in her practice to the Daily 5 and Daily 3 frameworks prescribed by Lambert that she used for reading and math. Without similar structures and expectations in place to support modifying instruction by interest and learning profile, these approaches to addressing variance in student needs and interests remained "not in place," although Nicole hoped to change that in spring 2015.

2(b) Summary. Nicole implemented many practices reflecting key principles related to all of the non-negotiable elements of a differentiated classroom, although she felt the demands of the first year posed obstacles that prevented her from implementing the model as she had anticipated as a preservice teacher. At the beginning of the school year, her primary focus was building a welcoming classroom community and establishing routines that allowed students to complete independent and small group work without teacher involvement. As she devoted increasing attention to her curriculum, she faced challenges surrounding maintaining the pace indicated in Lambert's curriculum map, designing clear learning goals for her units consistently, and making decisions about what to teach students who were significantly below grade level. For language arts, Nicole used results from a standardized assessment to determine student readiness and form her groups for instruction. In math, she used formal assessments like tests but also observed and took notes as individual students worked on problems or had one-on-one conferences with students to probe their thinking and look for misconceptions. Although she reported it was not happening as frequently as she wanted it to, modifying instruction based on student readiness was the norm in Nicole's language arts and math instruction, and Nicole hoped that her instruction the following spring would respond to student interest 
and learning profile as well.

\section{Research Question 2(c): How did contextual factors relate to Nicole's conceptions of the model and her teaching practice?}

When Nicole began teaching at Lambert, she encountered a host of factors involving the school's culture of practice, the nature of her particular position, and who the 21 students in her class were that shaped the ways in which her conceptions of differentiation were and were not reflected in her teaching practice during the fall of her first year. While a few of these factors challenged her implementation of differentiation, most supported her efforts to align her teaching practice with her philosophy of responsive instruction. Together, these factors created the complex context in which Nicole learned to work toward this alignment.

Culture of practice. When Nicole was hired by Oakhurst district in March 2014, she was told she would be teaching upper elementary classes at Williams Elementary (interview, 4/10/14), a school in an affluent area with students who were largely academically successful and with only around a third receiving free or reduced price meals (Oakhurst website, 2015). Nicole therefore completed the second half of the Differentiating Instruction course envisioning that the model would inform her work with this student population. However, Nicole was ultimately sent to Lambert instead, a Title I school with many "very needy" students living in chronic poverty, as well as students from middle class backgrounds (Nicole, interview, 8/25/14). During our interview, Nicole's mentor Sharon began her description of Lambert by saying, "The culture is here is one of diversity. Needs on all ends of the spectrum" (Sharon, interview, 10/20/14). At Lambert, students of color comprise two-thirds of the student body, and almost half of all 
students are identified as limited English proficient, with Spanish being their predominant native language (Oakhurst website, 2015). As a result, the school was in the process of phasing out their French immersion program and phasing in a Spanish immersion program (Sharon, interview, 10/20/14).

Nicole saw Lambert's cultural, socio-economic, and linguistic diversity as heightening the need for differentiation (interview, 8/25/14, 9/30/14). She characterized this diversity as supporting her attempts to implement differentiation, saying:

I can't sit here knowing these things, looking at how broad the range in my class is, and not do anything about it. I would like to think I don't need an incentive [to differentiate], but I have one, so there it is. (interview, 12/09/14)

In light of the diversity reflected at Lambert, the school and the district surrounded its faculty, especially first year teachers, with resources to improve their practice. Nicole reported, "I've gotten a lot of new teacher support" (interview, 11/14/14). During the fall of her first year in the classroom, Nicole was supported by a mentor, a "lead mentor" who oversaw the mentoring program, a literacy instructional coach, a math instructional coach, three more experienced third grade teachers, the assistant principal, and monthly district-level programming for new teachers (interview, 9/30/14). Nicole benefited from numerous formal structures designed to support her success.

Third grade team and professional learning community. Nicole was part of a team of seven third grade teachers, four of whom were first year teachers. The team also included the English for speakers of other languages (ESOL) teacher and the special education teacher assigned to third grade. One of the three more experienced teachers served as the team leader for their grade. The team usually met weekly for informal meetings led by the team leader. The focus of these meetings was curriculum and 
instruction: "making sure we're all kind of on the same pace" (Nicole, interview, 9/30/14) with the curriculum map and planning units. Once per quarter, the school hired substitutes for the third grade team so they could spend an entire day planning together (Nicole, interview, 9/30/14).

Twice a week, Nicole also had meetings with her professional learning community (PLC). This included the nine members of the third grade team, plus an instructional coach. On Tuesdays, the PLC focused on math instruction and was led by the math instructional coach. On Thursdays, it focused on literacy instruction and was led by the literacy instructional coach. Administrators would occasionally attend these meetings to present training sessions on various issues (Nicole, interview, 9/30/14).

Nicole found the members of these teams to be very collaborative, saying "everybody wants to work with people who are excited and willing to share" (interview, Nicole, $8 / 25 / 15$ ). She believed that this collaborative culture within the third grade team supported her implementation of differentiation because she was comfortable asking her peers for help when she did not know what to do (interview, 12/09/14).

Administration and mentoring. Nicole characterized the administration in her school as "very approachable" (interview, 9/30/14), but she did not have much formal interaction with administrators. In addition to the principal, Lambert had two assistant principals, with one assigned to the lower elementary grades and one assigned to the upper elementary grades. It was the assistant principal assigned to third grade who completed Nicole's evaluations. The only other interaction Nicole had with the assistant principal involved issues with parents. Nicole explained that in light of the school's "chain of command," she would look to other sources of support for "day to day practice" 
issues (interview, 9/30/14). Nicole's biggest source of support and guidance came from her mentor.

Sharon, who had been a teacher and then math resource specialist at the school for around 15 years, was Nicole's mentor. I interviewed Sharon to gain a broader perspective of Lambert's culture of practice and the nature of support given to first year teachers at the school because Nicole reported that Sharon had more influence on her curriculum and instruction than any other resource (interview, 9/30/14). Nicole met with Sharon one-on-one once a week for 30 minutes. Sharon structured these meetings to include a "debrief" in which Nicole described her experiences since their last meeting and a "look ahead" in which they discussed her plans for the coming week (Sharon, interview, 10/20/14). Topics they often addressed included instructional strategies, resources, substitute plans, the teacher evaluation process, and other challenges (Sharon, interview, 10/20/14). Sharon described the meeting agenda as being set half by her and half by Nicole, with Sharon encouraging Nicole to ask questions. For Sharon, the overarching purpose of these meetings was to ensure Nicole understood the message that: “I'm here for you. This is something I can help you with. It's communicating that I'm available, so she isn't wondering, can I ask her to?” (Sharon, interview, 10/20/14).

As Nicole's mentor and one of Lambert's math resource specialists, Sharon would occasionally visit Nicole's classroom during her math class to co-teach lessons, observe students who were struggling with conceptual understanding, give some students math recovery assessments, or work with small groups (Nicole, interview, 9/30/14; Sharon, interview, 10/20/14). Sharon initially suggested this kind of partnership with Nicole, but Nicole soon began reaching out to Sharon for this kind of support (Nicole, interview, 
9/30/14). Nicole explained:

A lot of that just comes out of our conversations, where it's like, I'm nervous about this, I'm struggling with this, and can you come look? And then that evolves into, well, why don't I come in and not just sit here but work with your students? So I would say at this point I would feel comfortable if she asked to come in, if she suggested, and I would also feel comfortable asking, is there a time this week that you can come into my class and help me with this? (interview, 9/30/14)

Nicole's comments suggested that she had received Sharon's message that Sharon was available to support Nicole in her instruction and that Nicole should feel comfortable asking Sharon for help.

The mentoring system in which Sharon and Nicole participated was structured by Oakhurst's formal policies and programs. Sharon had mentored two other teachers in previous years. In Oakhurst, mentors were only allowed to have one mentee per year (Sharon, interview, 10/20/14). The year before she became a mentor for the first time, Sharon had to complete a course on becoming an effective mentor and managing the administrative details of the mentoring process. She also had to take a refresher course on mentoring every two years to "streamline" her focus as an effective mentor (interview, 10/20/14). Three times per quarter, Sharon was required to complete a log describing the topics she had discussed with Nicole and indicating which of the criteria by which Nicole would be evaluated their discussions had addressed. Each school in Oakhurst also had a lead mentor who oversaw the mentoring program there. Nicole reported that, after her mentor, she had the most contact about her teaching practice with Lambert's lead mentor. The lead mentor, who was an experienced fourth grade teacher, matched new teachers with mentors at the school and ensured all of the mentors were completing the administrative requirements (Nicole, interview, 9/30/14). She also ran a meeting for 
Lambert's new teachers every month and acted like a "sounding board" for their questions and concerns, communicating that she had an "open door" policy (interview, 9/30/14).

District-level resources. Nicole described Oakhurst as a "resource-rich" district (interview, 1/27/14), and comments made by Nicole and Sharon during Phase 2 interviews supported this. This range of resources included everything from the financial resources to hire substitute teachers so that teams of teachers could spend a full day planning (Nicole, interview, 9/30/14) to providing teachers access to a huge volume of instructional materials including trade books for students, books on instructional topics for teachers, and an online clearinghouse of pre-made assessments and learning activities aligned to district grade level pacing guides (Sharon, interview, 10/20/14). It also included a district-wide training program for new teachers, which involved orientation sessions over the summer and monthly meetings.

It was at this summer orientation session for Oakhurst's new teachers that Nicole first heard differentiated instruction mentioned within the context of her new job. While she was initially concerned about how Oakhurst would define differentiation (interview, 8/25/14), she later recognized that the district used Tomlinson's model as its definition (interview, 10/20/14). While new teachers were instructed on differentiation during yearlong district programming, all teachers participated in a two-hour professional development session on differentiation presented by Tomlinson during the fall (Nicole, interview, 11/14/14). Much of this session focused on high quality curriculum grounded in essential understandings and, for Nicole, was a review of topics she had learned during the spring Differentiating Instruction course. Nicole was surprised to realize through this 
PD session that she knew much more about teaching for understanding than a highly experienced teacher on her team and provided her peer with some clarification on writing high quality understanding goals (interview, 11/14/14). In Oakhurst, it was expected that teachers would differentiate instruction, and its use was included prominently in the teacher evaluation criteria (Sharon, interview, 10/20/14).

Nature of position. Nicole was hired to teach on a third grade team at Lambert that had more first year teachers than experienced teachers. She was unsure about the nature of the relationship between this factor and her implementation of differentiation:

On the one hand, we're all very recent graduates...we're all very much on the same page in terms of philosophy, maybe not style of teaching, but differentiation is definitely something we were all given in our college programs because we're all new teachers, and that's where the field is moving toward. But at the same time, we're all new, and we're all running around like chickens with our heads cut off at times, so...there's a lot of survival mode going on. Differentiation and survival mode are not super compatible. (interview, 12/09/14)

Although the other new teachers on Nicole's team may have been more familiar with differentiation than some of the more experienced teachers, the new teachers in "survival mode" seemed less prepared to modify instruction. Her interactions with peers who were “running around like chickens with their heads cut off" were not particularly supportive of her attempts to differentiate, despite her colleagues' familiarity with the model.

The unique aspect of Nicole's position on the third grade team was that Lambert's third grade ESOL specialist, Debbie, was stationed inside Nicole's classroom. Debbie pulled students out of Nicole's class and other third grade classes for English instruction at a large table in the back of Nicole's classroom throughout the day (interview, 8/25/14; observations, 9/30/14, 10/20/14, 11/14/14, 12/09/14). While Nicole began the year concerned about how the dynamic of sharing her classroom space with another teacher 
would work (interview, 8/25/14), at the end of the fall, she concluded that having Debbie enhanced her implementation of differentiated instruction since Debbie could provide small group instruction and individualized support for students with limited English proficiency in her class (interview, 12/09/14).

Nicole's students. In January 2014, during our first interview, Nicole had shared that in an ideal world, she would love to teach students who all had supportive home environments and were working on grade-level (interview, 1/27/14). The students Nicole taught in her first year at Lambert did not meet this description. Instead, her students were "very low academically" (interview, 9/30/14). Nicole described responding to their significant academic needs as a struggle, saying, "The things that we're expected [to do], I'm like, oh, but I don't know, 'cause they're still two grade levels or more below in reading, and this is a nice idea, but shouldn't we get them up to speed in writing first?" (interview, 9/30/13).

Nicole's class of 21 students includes 19 students who are non-white. Seventeen students are Hispanic, with one being from Peru, one from Mexico, and the other 15 from El Salvador. One student is Nepalese, and one is African-American. However, Nicole did not describe her classroom as culturally diverse, explaining, "It's diverse in the institutional sense of, look how many non-white students you have, but I have a big majority culture. It's just not white culture” (interview, 12/09/14). Thus, Nicole ultimately did not see cultural diversity as a factor compelling her to differentiate in her classroom (interview, 12/09/14).

For all 17 of Nicole's Hispanic students, Spanish is the primary or only language spoken at home. Sixteen of those students are identified as having limited English 
proficiency, with 11 of them scoring below WIDA level 4. Nicole had a much higher number of ESOL students than other third grade classes, which had a quarter to a half of their students with limited English proficiency. In addition to limited proficiency with English, Nicole, her mentor, and the special education specialist believed some students in Nicole's class also had some significant learning disabilities, for which they would probably be evaluated later in the year (interview, 9/30/14). During observations, I watched numerous examples of Nicole working with students who struggled to memorize a handful of discrete facts or think conceptually at basic levels. For example, during one observation of science and social studies lessons, I observed (a) Nicole provide extensive scaffolding through one-on-one conversations with a student who could not recall the topic of a project he had just spent 20 minutes working on, (b) a student who could not guess a reason why a group of people would want to build a large wall at the edge of their lands, and (c) a group of students who could not identify where their parents go to buy the vegetables they eat at home (observation, 10/20/14). "The need [for differentiation] is in front of me," concluded Nicole (interview, 12/09/14).

Factors with greatest influence and 2(c) summary. During our interview in December 2014, I asked Nicole to reflect on the relationship between her implementation of differentiation and many factors involving Lambert's culture of practice, the nature of her position on the third grade team, and her students that she had mentioned in previous interviews and reflections (see Appendix I). Nicole considered each of these factors and classified them as having supported her implementation of differentiation, challenged her implementation, or played no role in her implementation, or she indicated that she was uncertain as to whether it was related to her implementation. After she had explained her 
responses to all factors, I asked Nicole to consider each factor she had characterized as having influenced her implementation, either by supporting or challenging it, and to identify the three factors that had played the most significant roles. All three factors she selected had supported her implementation of differentiation. In descending order of importance, they were the support she received as a new teacher, her students' needs, and her participation in the study.

New teacher support. Nicole explained her choice to identify the support she received as a new teacher as having the strongest relationship to her implementation of differentiation by saying that "even with all the great ideas I had about differentiation and wanting to do it, it would be a million times harder to implement that as a brand new teacher if I was in a school that didn't care about it" (interview, 12/09/14). In drawing this conclusion, Nicole pointed specifically to the support she received at Lambert for differentiating instruction regularly in her classroom practice, not just to new teacher support in general. Nicole perceived this support for differentiation as taking two forms: beliefs and practice. First, Nicole was surrounded by people who saw differentiation as important. She described:

All of these people are on the same page in terms of what's important, and that is differentiation and meeting the students where they're at and respectful tasks, and all those things are things that I've heard from them, which is like, great! I believe in that too. (interview, 12/09/14)

What Nicole described with these comments was a set of common beliefs about differentiation. Not only did her peers, mentors, and administrators share the same knowledge that Nicole did in terms of defining differentiation in the same way, they also believed that it was a priority for instruction in their school. Second, they acted on those beliefs and supported Nicole in doing so as well. Nicole continued: 
But then also in terms of actually doing it, I've had coaches in my room, I've had my mentor in my room, and that allows them to teach a lesson and me to see it, or if they're targeting working with a group to their specific needs and then I take a different group, just physically having two bodies in the room allows me to do more.

Nicole recognized that, as a new teacher who wanted and was expected to implement differentiated instruction in her new classroom, she was greatly supported both by sharing common beliefs about the model with her colleagues and by being guided in targeted and specific ways to use the model effectively in her own practice.

Student needs. The contextual factor Nicole felt had the second strongest relationship to the implementation of differentiation involved the significant needs she recognized in her 21 students. Interestingly, Nicole felt that having such prominent academic needs in her class supported rather than challenged her implementation of differentiation because she felt their presence compelled a response from her. She explained:

The actual real live little humans I'm responsible for require it, and it's so much easier to see that once you meet them and work with them, and you're like, I just want you to do better. What can I do? Anything I can do to help you do better! (interview, 12/09/14)

Thus, the substantial needs reflected by her students seemed to create a sense of urgency in Nicole to respond. This was an urgency that could not exist in the same way as a preservice teacher when thinking about her future classroom in the abstract; seeing the students each day in front of her was the catalyst for these feelings. Nicole's comments suggested she adopted a whatever-it-takes mentality to address those needs and support student growth. 
Study participation. Last, Nicole identified her participation in Phase 2 of this research study as having the third strongest relationship to her implementation of differentiation and indicated that this factor had also supported her implementation.

This was a factor that Nicole had not addressed directly until this December interview, although comments she had made in previous interviews had hinted that Nicole remained conscious of the fact that her implementation of differentiated instruction was being studied by a researcher. Three examples from our interviews illustrate this high level of awareness in Nicole. First, during the November interview, I asked a follow-up question to our October interview in which she had said she hoped to modify her instruction based on student interest in an upcoming social studies unit on ancient Egypt. In November, when I began to ask her how her Egypt unit had gone, she started smiling broadly at me. I commented that she was smiling, and she said, "No, and I knew you were going to ask that! I was like, oh!” Although she was still smiling, Nicole then made a grunting sound, formed her hand into a fist, and moved her fist swiftly through the air, in an apparent expression of mock frustration (interview, 11/14/14). Nicole went on to explain that she had not modified instruction during the unit, and her tone of voice and facial expression suggested she was slightly disappointed to share this news with me. In a second example, when Nicole explained that differentiation was not happening in her classroom as much as she would want it to, she commented, "And I hate to say that, because it's going to go in your dissertation" (interview, 12/09/14). And in a third instance, with a smile and a chuckle, she joked that it felt like I was grading her when I was in her classroom (interview, 12/09/14). 
Ultimately, Nicole characterized her Phase 2 participation as a significant factor in supporting her implementation of differentiation. Nicole's comments pointed to two reasons for this. The first was that being in the study provided an "incentive" for her to differentiate (interview 12/09/14). She explained her thoughts:

I can't have Hilary show up and me being like, I don't believe in differentiation any more. Not that that would happen.... While you're totally non-evaluative and you're not giving me a grade, it's still somebody writing a dissertation about differentiation coming in and looking at my classroom, and that puts you, I think anytime somebody comes in to watch you in your classroom, that kind of makes you, or for me at least, it makes me want to be on best teacher behavior. You want to show all the good things you know you're doing. (interview 12/09/14)

Put simply, being observed as part of a research study made Nicole want to do her best, particularly in the area of teaching on which she knew the study focused.

The second reason Nicole identified as to why participating in the study was a significant factor in supporting her implementation of differentiation involved her thinking:

I think it made me, it changed how I thought about [differentiation] in my brain. I feel it's been very, very consciously on my mind, and while I would like to think that it would have been despite not being in the research study, I don't know, because I was never not a participant....I don't think there's any way to not be thinking about it if I know that I'm in the research study. (interview, 12/09/14)

Being in a study about differentiated instruction in which she was interviewed and observed once every month meant that differentiation was at the forefront of Nicole's thinking on a regular basis, particularly since the interviews prompted Nicole to reflect on her current practice and compare it to her expectations as a preservice teacher. While Nicole thought her implementation of differentiation would not have looked different if she had not been in the study, she was unsure of how influential her participation in the 
study had been on her practice (interview, 12/09/14). Connections to observer effects discussed in the Limitations section of Chapter Six are evident. 


\section{CHAPTER SIX}

\section{CROSS-CASE THEMES, ASSERTIONS, \& IMPLICATIONS}

This longitudinal multicase study explored two phenomena: (a) how preservice teachers enrolled in a course on differentiated instruction in a teacher preparation setting make meaning of Tomlinson's $(1999,2014)$ model of differentiation, and (b) how teachers who completed a course on differentiated instruction in a teacher preparation setting make meaning of Tomlinson's model of differentiation during the fall of their first

year in the classroom. Findings about Karen's experiences described in Chapter Four and Nicole's experiences described in Chapter Five serve as the foundation for the cross-case themes and assertions presented in this chapter. After detailing the study limitations, I conclude the chapter by addressing the implications of the cross-case assertions for teacher educators.

\section{Cross-Case Themes \& Assertions}

This section presents the results of cross-case analysis. Because the goal of a multicase study is to gain a better understanding of the phenomenon of study, after cases are studied individually, the cases are then considered together (Stake, 2006). I begin this section by presenting cross-case themes (Stake, 2006) that address each of the study's five research sub-questions and illuminate the phenomena of study. These themes build upon the findings from the single cases synthesized above. I then identify four key assertions (Stake, 2006) that address broad issues extending across both study phases. 
The significance of these assertions is discussed in light of the literature reviewed in Chapter Two.

(1a) What are preservice teachers' conceptions of the model when they first enter the course, if any, and how do their conceptions change during the course, if at all?

Participants began the course with existing knowledge and beliefs of differentiated instruction, with Karen's conceptions being more consistent with Tomlinson's model. As the course progressed, the evolution of their conceptions included deepening their understanding of the model, resolving their misconceptions, developing or refining conceptions of specific model components, recognizing the interdependent relationships among model elements, and anticipating a role for differentiation in their future practice in light of their beliefs about its potential efficacy. This process was not linear or predictable. It involved comparing, evaluating, and connecting prior knowledge and beliefs about differentiation and other ideas about teaching and learning with new information encountered during the course.

Significantly, both participants ended the course anticipating that their newly developed conceptions would play a role in their future practice, and they each hoped this would involve differentiation serving as the lens through which they thought about teaching and learning. They viewed differentiation as an ethical obligation and considered it important to their own professional success, as well as the academic success of their students. 


\section{(1b) How do factors external to the course relate to preservice teachers' conceptions}

\section{of the model?}

Varied relationships existed between the participants' conceptions of the model and aspects of their personal backgrounds and teacher education experiences. Some factors enhanced participants' conceptions, while others detracted from them.

Personal background. Both participants saw their existing philosophy of teaching and learning as highly compatible with the philosophy of differentiation; neither identified any areas of conflict between the two. This compatibility supported their conceptions of differentiation. Learning more about differentiation provided opportunities for them to gain clarity surrounding their own beliefs about teaching and learning. For Karen, this process involved feeling reaffirmed in her prior beliefs and viewing them as the foundation for understanding differentiation, while Nicole saw differentiation as giving her the verbiage to describe her existing beliefs and a lens or organizing framework through which to view them.

Neither participant had seen meaningful examples of differentiation in their own K-12 experiences. Nicole viewed this factor as having no significant relationship to her conceptions of differentiation, but Karen viewed this as enhancing her conceptions in that it gave her countless examples of what differentiation does not look like. Both recognized the importance of mindset for a teacher's work to differentiate instruction and for a student's outlook on school.

Teacher education experiences. Both participants viewed their prior education coursework as a factor that significantly detracted from the conceptions of differentiation they developed during Phase 1. The exception to this was a curriculum design course 
that Karen had taken with Tomlinson during a previous semester. Neither participant saw differentiation fully modeled during their field placements, as practice involving at least some of the key elements of the model was absent in those contexts.

\section{(2a) What are teachers' conceptions of the model as they first enter the classroom, and how do their conceptions change during the fall, if at all?}

As they entered the classroom at the beginning of the fall, neither participant reported gaining new knowledge of differentiation over the summer, but both suggested that their beliefs about the model had changed slightly. This was because, just before the start of school, both had become aware of the readiness needs that would be present in their classes. For Karen, this meant learning from her students' parents about the wide range of readiness her kindergarten class would include, especially in reading. For Nicole, this meant learning about the large number of students with limited English proficiency in her third grade classroom and the significant socio-economic challenges of half of Lambert's student population. With this information in mind, both participants saw the need for differentiation as heightened. Their belief in the importance of the model was therefore reinforced.

As the fall progressed, both viewed implementing differentiation as challenging. Each participant recognized that, as a first year teacher, she would need to prioritize her attempts to respond to student needs, and both decided to focus on modifying instruction by readiness based on the belief that it was the most important form of differentiation for supporting academic success. 


\section{(2b) What is the relationship between teachers' conceptions of the model and their}

\section{teaching practice?}

Numerous aspects of Nicole's and Karen's teaching practice reflected their knowledge and beliefs about differentiation, and their experiences in the classroom paralleled one another's in many ways. Karen and Nicole both differentiated instruction with moderate fidelity to Tomlinson's model and with persistence, having entered the classroom with the knowledge, skills, and dispositions needed to do so. Both quickly established supportive learning environments and taught students to execute classroom procedures effectively, while both experienced challenge in applying principles of high quality curriculum and assessment. For Karen, these challenges were due to the unfettered freedom she had at Newland. In contrast, Nicole grappled with school or district requirements that conflicted with how she wanted to respond to student needs. Both did not modify instruction by interest or learning profile much during the fall, and both focused their attention on modifying instruction by readiness in language arts and math, rather than social studies or science. Both used formal assessment data to put students into long-term readiness groups. Both used the Daily 5 literacy instructional framework to structure differentiated language arts rotations. Nicole used the Daily 3 math instructional framework and reported no problems structuring rotations in that class, but Karen experienced significant uncertainty about structuring her math time.

Both teachers reflected that they were not yet implementing differentiation in the way they had hoped and anticipated as preservice teachers. In this way, they used their existing knowledge, skills, and beliefs about differentiation, which they attributed largely to the Differentiating Instruction course, to evaluate their current practice and to plan for 
their next stages of growth. Interestingly, when reflecting on "differentiation" in their classrooms during the fall, the participants did not discuss the elements of the model such as learning environment and classroom management which were well established, and which they had identified as important foundations to effective modification of instruction. Instead, they focused almost exclusively on the fact that they were not modifying instruction by interest or learning profile and that they were not yet satisfied with their modification of instruction by readiness.

\section{(2c) How do contextual factors relate to teachers' conceptions of the model and their}

\section{teaching practice?}

Nicole and Karen joined faculties at significantly different schools of employment, yet differentiation was an established expectation in both settings. While Nicole benefited from a range of structured opportunities for deep support of her teaching practice in general and of differentiation in particular, Karen's determination to improve her practice related to differentiation led her to seek support outside of her school.

One cross-case theme stood out as unanticipated based on the existing literature. It involved the participants' identification of the needs of individual learners in their classrooms as a contextual factor significantly related to their conceptions of differentiation and their teaching practice. Who the teachers perceived their students to be, and in particular the readiness needs their students presented, not only caused the participants to see differentiation as even more important than they had understood as preservice teachers. It also served as a critical impetus for action based on that belief. Nicole characterized this as one of the most influential factors supporting her implementation, second only to the volume of effective new teacher support she received. 
In contrast, Karen saw this factor as the most serious challenge to implementing differentiation she faced, yet it still served as a catalyst for responding to student need in meaningful ways.

The differences between the participants' responses to this issue may be attributable to the support they did or did not receive in how to structure their instruction in response to those needs. At Lambert, Nicole received significant support of her attempts to address the readiness needs of English language learners and students who may have learning disabilities, and she in turn viewed these student differences as supporting her implementation differentiation. At Newland, Karen received minimal support of her attempts to address the readiness needs with literacy skills and number sense of her students performing at advanced versus on-grade levels, and she saw these student differences as challenging her implementation of differentiation.

I conclude this section by presenting assertions (Stake, 2006) that address broad issues building upon the cross-case themes and by discussing their significance in light of the literature. As with all elements of the study's analysis and interpretation, the assertions are framed through the situative perspective on teacher cognition (Putnam \& Borko, 2000).

Assertion 1 - The challenges of undoing and retraining: The participants'prior coursework detracted from their developing conceptions of differentiation because it resulted in their needing to "undo" (Nicole, interview, 4/06/14) and "retrain" (Karen, interview, 5/01/14) their thinking about the model.

Differentiation had been mentioned throughout the participants' prior education coursework and received uniformly positive treatment. However, Karen and Nicole felt 
that when these previous courses addressed the topic of differentiation, particularly in their introductory curriculum and instruction courses, its treatment had not reflected a conception of differentiation as a systematic, structured way to respond to student differences. The participants reported that their prior coursework had not emphasized that differentiation is a philosophy, leaving Nicole to conclude that it is a checklist of strategies and Karen to conclude it is an instructional model limited to curriculum, instruction, and assessment. Neither recognized from their coursework that modification of instruction by readiness, the core element of the model, is an essential component of differentiation. Both entered the Differentiating Instruction course with conceptions of differentiation that, to some degree, mirrored its treatment on their program's lesson plan template: a small box to be checked off when a differentiated strategy was added on to regular instruction.

Each of the courses participants took during their teacher education programs represented a different context of learning. The situative perspective of cognition reflects the belief that learning cannot be separated from the contexts in which it is gained and used (Greeno, 1997, 2003). Teacher education curriculum grounded in this perspective “emphasizes understanding, reasoning, and connections among important ideas, all intertwined with the settings in which this knowledge will be used" (Borko \& Putnam, 1996, p. 674). One aspect of this approach to teacher education curriculum is providing students with opportunities to "integrate or coordinate content across courses" (Borko \& Putnam, 1996, p. 674). The design of a teacher education program can encourage or discourage students to connect content across courses. 
In the present study, participants suggested that, in their teacher education program, they had benefitted from receiving a coherent and strong core message throughout their all coursework reflecting the beliefs that knowing students as individuals, attending to their needs, and making instruction student-centered were paramount in teaching. In this way, their courses shared common conceptions of teaching and learning based on clear values, which generally facilitated connecting content across courses. However, participants felt they received conflicting or unclear information from the treatment of differentiated instruction in different contexts during their coursework. While the core values reflected in their teacher education program were tightly aligned with the philosophy of differentiation, the participants felt that stronger coordination of specific content across courses in this particular instance would have been more supportive of their developing conceptions of the model.

Assertion 2 - The three pillars for encouraging implementation: To encourage the implementation of differentiation, participants needed a community of practice featuring (a) a shared vision of differentiation; (b) structured, deep support of differentiated practice; and (c) the flexibility to respond to student needs.

When Karen and Nicole entered their schools of employment, they quickly recognized the school communities' expectation that differentiated instruction would be reflected in their practice. This expectation was communicated through formal structures, such as evaluation criteria and orientation programming, and through less formal means, such as interactions with colleagues or mentors. As a result, neither participant experienced "problems of enactment" (Kennedy, 1999) resulting from the "two-worlds pitfall" (Feiman-Nemser \& Buchmann, 1983) of negotiating among 
conflicting norms and messages across preservice and in-service settings (Thompson et al., 2013). When Karen and Nicole taught through a differentiated approach in their schools, to a large extent, they engaged in "integrated appropriation" (Thompson et al., 2013): They maintained their preservice pedagogical beliefs and implemented multiple forms of complex practice they learned in their teacher education program (Thompson et all, 2013).

Throughout the fall, the participants engaged in situative learning within their communities of practice (Wenger, 1998). This conception of learning views it as the process through which a learner takes part in and gains proficiency with the practices of a particular community (Wenger, 1998). From this situative perspective, novices begin on the periphery of the community, and as they become more adept with its practice and are enculturated into the community, their learning moves them into a more internal, integrated position (Wenger, 1998). This learning generally occurs through interactions with people, ideas, and materials in a range of complex situations, when participating in the situation makes the individual a more effective participant in the valued social practices of the community (Greeno, 1997). According to this theory, teacher learning in a community of practice involves acquiring the language, knowledge, skills, and dispositions needed for successful membership in their classroom, department, school, or district (Borko, 2004; Wenger, 1998).

Nicole and Karen joined communities of practice in which differentiated instruction was a valued practice. Through their interactions with students, colleagues, curricular documents, and other materials, the participants recognized the knowledge, skills, and dispositions they would need for successful membership in Newland 
Academy, Lambert Elementary, and Oakhurst County Public Schools, including those related to differentiation. Considering the totality of the participants' experiences working with differentiation in their different contexts, it appeared that, to implement differentiation effectively, these new teachers needed a community of practice characterized by (a) a shared vision of differentiation; (b) structured, deep support of differentiated practice; and (c) the flexibility to respond to student needs.

Vision. Both participants entered communities of practice in which their colleagues shared their knowledge of differentiation, shared their beliefs about differentiation, and put their knowledge and beliefs into practice regularly through their instruction. From the time she interviewed with Newland in spring 2014, Karen was aware that teachers at the school defined differentiation the same way she did through Tomlinson's model, valued it as a powerful philosophy to meet the needs of students, and practiced differentiated instruction in their multiage classrooms. Nicole did not begin the fall with the same certainties, but quickly recognized the same was true in her school and district of employment. Neither participant experienced the dissonance common among new teachers when their beliefs about teaching and learning developed during teacher education experiences conflict with local practice norms (Raymond, 1997). Instead, their images of differentiated practice in their own classrooms to a large extent conformed to local practice norms. Exposure to instructional norms through colleagues' actual classroom practice can affect the instructional practices of new teachers (Frank et al., 2008; Youngs et al., 2012). It is important to note that Nicole and Karen appeared to be exposed to instructional norms involving differentiation largely through interactions with colleagues rather than direct observation of differentiated practice. Because of the 
compatibility between participants' and their colleagues' knowledge of differentiation, beliefs about differentiation, and differentiated classroom practice, Nicole's and Karen's situated learning in their new communities of practice did not require them to acquire extensive new language, knowledge, skills, and dispositions needed for successful membership (Borko, 2004; Wenger, 1998). The participants shared the same vision of differentiation with the other members of their community of practice.

Support. The nature and level of support new teachers receive through interactions with mentors, colleagues, and administrators can affect how they are socialized into their schools of employment (Coburn \& Russell, 2008; Feiman-Nemser, 2001; Frank et al, 2008; McGinnis et al., 2004; Youngs, 2007; Youngs et al., 2012) and to what extent they implement ambitious practices learned during teacher education (Cady et al., 2006; Flores, 2006; Prescott \& Cavanagh, 2008; Steele, 2001). The literature examining student teachers' conceptions of differentiation also suggests that establishing collegial relationships with colleagues involving open communication about teaching practice supports novices' efforts to differentiate (Tricario \& Yendol-Hoppey, 2012), while a lack of support of differentiation from other community of practice members is a barrier to new teachers addressing academic diversity (Tomlinson et al., 1994).

Nicole cited the extensive new teacher support she received as the most important factor supporting her implementation of differentiation, while a lack of effective support from within her community of practice led Karen to seek support elsewhere. The deep support Nicole received was characterized by its high level of structure and its strategic design to anticipate and respond to the many needs of novices. Her relationship with her 
mentor allowed her individual concerns to be addressed. She received support from many different colleagues through regular, recurring interactions at times that were protected for these purposes. In contrast, Karen had no such regular supportive interactions with colleagues at protected times, and when she did receive information from a colleague in response to a question, it seemed not to be helpful. Karen felt so strongly that she needed guidance in her attempts to differentiate that she sought help beyond her community of practice from numerous sources, spending large amounts of her limited time and energy in the process.

Flexibility. Last, the element of the flexibility to respond to student needs appeared important in implementing differentiation. The freedom Newland Academy gave its teachers to teach as they believed offered them flexibility in making decisions about curriculum content and pacing, in adjusting daily schedules, in choosing instructional practices, and in using non-traditional approaches to meet student needs, such as taking a student for a walk around the block to settle. Nicole did not experience the same kind of flexibility at Lambert. Instead, she described working within the tension caused by juxtaposing what her students needed and what external mandates required her to do. Nicole was generally expected to follow the pace outlined in Lambert's curriculum maps, even when she felt her students needed additional time to master content. She also graded formative assessments to justify report card grades, despite the belief she developed in the Differentiating Instruction course that most formative assessments should not be graded. In these ways, she engaged in "strategic compliance" (Lacey, 1977), outwardly complying to institutional constraints while maintaining inner reservations about these decisions. 
The issue of flexibility is prominent in the literature on factors that influence the socialization of new teachers and their implementation of ambitious practices, referencing teacher flexibility in terms of curriculum and assessment practices (Grossman et al., 2000; McGinnis et al., 2004; Prescott \& Cavanagh, 2008; Steele, 2001) and use of time in the face of pressures to cover content (Prescott \& Cavanagh, 2008; Raymond, 1997). In her study examining preservice teachers' developing conceptions of differentiation in a secondary science methods course, Goodnough (2010) found that preservice teachers anticipated that a lack of flexibility in decision-making surrounding instruction and assessment in their schools of employment would challenge the implementation of differentiation, an assertion supported by the findings of the present study.

While both participants became members in communities of practice that largely reflected a shared vision of differentiation, Karen lacked structured and deep support of her differentiated practice, and Nicole's teaching context sometimes did not offer the flexibility in responding to student needs she desired. Although Karen and Nicole were able to begin to implement differentiation in their practice, both encountered challenges involving one of these three elements that made their efforts more difficult. Because each element functioned as a pillar of support buttressing new teachers' implementation of differentiation, having one pillar that was not as well-established left the participants feeling less successful in their practice.

Assertion 3 - See it to do it: Not having seen differentiated instruction modeled in varied contexts detracted from both participants' conceptions of the model and challenged one participant's implementation. 
Throughout Phase 1, Karen and Nicole often spoke about not "seeing" what differentiation "looks like" (e.g., Karen, interviews, 1/27/14, 5/01/14; Nicole, interviews, 1/27/14, 2/24/14, 4/06/14; Nicole, reflection 2/14/14). Neither one recalled seeing Tomlinson's model of differentiation used during their own K-12 experiences, nor did they recall seeing it modeled by any of their university instructors other than Tomlinson. During student teaching, Karen's CI had not modeled many elements of differentiation. In Belfast, Nicole's CI had not modeled any aspects of Tomlinson's model of differentiation. Nicole felt that her local student teaching CI had done an excellent job modeling establishing a supportive learning environment and classroom routines at the beginning of the year, providing Nicole with a clear image of how to enact these practices. However, this CI did not explicitly model many of differentiation's principles involving curriculum, assessment, and instruction. Nicole believed that the principle of teaching up would be difficult to implement in her own practice because she had never seen it implemented in another classroom. Thus, both participants began the Differentiating Instruction course believing that they did not know what effective differentiation looks like.

As they gained knowledge and skills from the course, Nicole and Karen began to develop a clearer image of how the principles of differentiation might look in action in a classroom at their grade level; however, this process proved difficult without specific, explicit images of the practices from previous learning contexts to which they could connect these ideas. During Phase 2, this posed a particular problem for Karen, who could not envision the logistics of structuring differentiated learning rotations in math. While Nicole's mentor was available to co-teach lessons or to teach Nicole's class while 
Nicole observed, Karen had no such regular opportunities. While Karen could have asked to observe in the classroom of her mentor, a third and fourth grade teacher, Karen sought a lower elementary model for her practice. The closest Karen seemed to come to this was looking at online pictures of learning centers in other kindergarten classrooms.

This issue of the difficulties involved in not having a clear image of how a complex instructional approach would be enacted in the classroom is related to two key topics addressed in the literature. The first is the modeling of best practices by university instructors. According to Tom (1997), the first principle of an effective teacher education program is that "The program faculty and the curriculum of a teacher education program should model the image(s) and skills of teaching that the faculty desires to foster among students in the program" (p. 102). This practice is particularly important if a program intends to prepare its teacher candidates to teach from a constructivist paradigm (Tom, 1997), as preservice teachers benefit from making meaning of the practice by experiencing it. Additionally, a situative perspective of teacher learning suggests that preservice teachers must engage in the experiences that university instructors hope they will design in their future classrooms (Borko \& Putnam, 1996). With differentiated instruction in particular, Santangelo and Tomlinson (2012) suggest that some instructors may not implement a comprehensive model of differentiation in their instruction of preservice teachers in that they neither teach it as content nor model it and debrief with students about its use. Doing so would give preservice teachers the opportunity to experience differentiation from the student perspective, allowing them to construct meaning from the principles and practices by experiencing them (Tom, 1997). Santangelo and Tomlinson's (2012) attention to the importance of debriefing with 
students is noteworthy in that it suggests students should have opportunities to reflect on and discuss with instructors the ways in which differentiation is being modeled in the course.

The second key topic related to the challenges participants faced in not having a clear image of differentiation involved not having seen it modeled in field placements, which can give preservice teachers the opportunity observe differentiation from the teacher perspective. In his work on communities of practice, Wenger (1998) also identified the key concept of alignment, or the nature and degree of shared or complementary aims among varied learning contexts. Researchers have frequently emphasized the importance of achieving this alignment and avoiding structural fragmentation (Zeichner \& Gore, 1990) in teacher education program designs to the greatest extent possible (Barone et al., 1996; Borko et al., 2000; Borko \& Putnam, 1996; Brouwer \& Korthagen, 2005; Cavanaugh \& Prescott, 2007; Feiman-Nemsor, 2001; McDonnough \& Matkins, 2010; Wideen et al., 1998). In their longitudinal study of novice secondary science and social studies teachers, from their teacher education program through their second year of teaching, Nolen, Horn, Ward, and Childers (2011) considered this concept of alignment in their examination of how their participants learned to teach. Drawing on Holland, Lachiotte, Skinner, and Cain's (1998) theory of figured worlds, Nolen and colleagues (2011) identified fields of practice in which the novice teachers in their study learned to teach. They called these fields TEPworld, Fieldworld, and Realworld. In TEPworld, or teacher education program world, their participants were students. Their learning was directed by their instructors. In Fieldworld, participants were student-teachers. This role was peripheral and temporary. 
In Realworld, the participants were first year teachers. The researchers described the relationship between TEPworld and Fieldworld by saying: "As novices move back and forth across the boundary between those two figured worlds, they often need to negotiate the meanings and value of particular practices promoted in one world with their meanings and value in the other" (Nolen et al., 2011, p. 91). These negotiations are especially prominent in preservice teachers' meaning-making processes when the meanings and values promoted in the two worlds are not aligned (Wegner, 1998).

If these constructs are applied to the present study, it appears that Nicole and Karen engaged in minimal negotiations between TEPworld and Realworld with respect to differentiated instruction because alignment existed between these two figured worlds. However, making meaning of their experiences in TEPworld and Fieldworld as they related to differentiation required negotiation, as these experiences were not fully aligned (Wegner, 1998). Although the participants saw some practices of differentiation modeled in Fieldworld, it appeared they did not see enough practices used frequently and effectively or discuss the use of those practices with CIs to gain a sense of what the model might look like in action. Additionally, when Karen entered Realworld, she experienced what some participants in Tomlinson et al.'s (1997) study of novice teachers' progression toward differentiated practice did as well: "a lack of models for how it would look" (p. 280) in her induction setting. The researchers reached the same conclusion suggested by the present study:

If novice teachers are expected to become architects of inclusive communities of learning, this study suggests that it will be necessary for them to develop images of classrooms [emphasis added] where...students are engaged with tasks that are...specifically designed to ensure that each student grows every day. (Tomlinson et al., 1997, p. 280) 


\section{Assertion 4 - Mindset matters: As participants made meaning of differentiation,} mindset served as a key lens through which they viewed themselves, their students, and their practice.

Throughout the study, the role of mindset (Dweck, 2007) in the participants' meaning making processes was striking. Karen's attitudes and actions seemed to epitomize a growth mindset. This was evidenced by the way she conceptualized learning as a process and responded to failure. It applied to how she thought about herself and her students as learners, and to how she taught her students. As a preservice teacher, Karen believed that she would inevitably make mistakes in her practice as she learned to lead her own classroom, but when she considered these future missteps, her focus was on learning from them to make her practice more successful. Once she entered the classroom, this was exactly the attitude she adopted. Although she gained comfort from the fact that she always gave her work her best and recognized her growth during the fall, she constantly sought out ways to improve. Her teaching practice also demonstrated numerous examples of how she encouraged her students to develop growth mindsets. Additionally, she worked in a school of employment with a significant focus on the importance of a growth mindset for teachers and students. Karen saw her mindset as the most important supporting factor for her implementation of differentiation. In contrast, Nicole did not yet have a fully growth mindset. Like Karen, she believed she would inevitably make mistakes in her future practice, but her focus was on preparing to deal with the disappointment they would cause her and on remaining willing to try again. Through highly self-aware comments, Nicole connected her mindset to her own educational experiences and considered the many steps she might take as a classroom 
teacher to ensure that a fixed mindset was not reflected in her interactions with students. Both participants were committed to viewing students as having malleable intelligence and treating them accordingly.

Limited research has examined preservice and inservice teachers' beliefs about intelligence. This gap in the literature is significant for several reasons. Students with growth mindsets are generally more academically motivated and perform at higher levels than those with fixed mindsets (Blackwell, Trzesniewski, \& Dweck, 2007; Dweck, 2000). Additionally, teachers' beliefs about intelligence can influence students' beliefs about intelligence (Oakes, Wells, Jones, \& Datnow, 1997; Watanabe, 2006), and this may in turn influence student achievement and motivation (Dweck, 2000). Specifically, connections have been identified between teachers' mindsets and their teaching approaches (Leroy, Bressoux, Sarrazin, \& Trouillod, 2007; Southerland \& GessNewsome, 1999). Teachers with growth mindsets tend to have higher self-efficacy, which is related to effectiveness in the classroom (Leroy et al., 2007).

The literature on the role of preservice teachers' beliefs in making meaning of educational philosophy or practice also has not yet examined the role of mindset in depth. In their case study of three student teachers in an elementary education program with a heavy focus on differentiation, Tricario and Yendol-Hoppey (2012) did note that one of the learning conditions that supported participants' efforts to differentiate during student teaching was an openness to receiving feedback and applying it in future lessons. It is possible that this condition was reflective of mindset.

Similarly, the literature on factors that may influence new teachers' implementation of ambitious practices they learned in teacher education programs has not 
yet considered mindset as a potential factor in depth. Cavanagh and Prescott (2007) and Prescott and Cavanagh (2008) did note that their participants' willingness to try to implement a practice a second time when not executed successfully the first time varied, but the authors did not name a personal characteristic that might explain this. Again, it is possible that their participants' responses of willingness or hesitancy in the face of initial failure may be indicative of mindset. Clearly, more research is needed to examine mindset as a potential influence on novice teachers' development of conceptions of differentiation or ambitious practices.

\section{Limitations}

This study had several limitations that should be considered when interpreting the findings. First, data were gathered primarily through four interviews in the spring, five interviews in the fall, and four classroom observations in the fall. I felt that it would have been unreasonable to ask for more of the participants' time, especially as first year teachers, and ethical considerations required me to keep in mind the costs borne by participants (Miles \& Huberman, 1994). However, making meaning of a complex philosophy is a constantly evolving process, involving intricate twists and turns in interpretation and an ebb and flow of clear understanding. A learner's thinking one day may be significantly different the next as new knowledge is encountered or new experiences occur. Thus, gathering data from participants at particular moments in time could not ensure a holistic understanding of this complex process. Although my weekly informal observations and my analysis of course assignments that were spread throughout the course during Phase 1 made a holistic understanding of the process much more likely, there is no guarantee that a full picture of participant meaning-making could be developed based on the time-points at which data happened to be gathered. The second 
and third limitations involved my working relationships with the participants during Phase 1 as described above in the Researcher as Instrument section and Differentiating Instruction Course subsection.

The second limitation was that my working relationship with the participants may have affected the nature of my interactions with them, how I analyzed the data, and how I interpreted the findings in ways of which I am unaware, despite my ongoing attention to these issues through structured opportunities for reflexive thinking and writing. In response, I worked to maintain a focus on the meaning-making perspectives of the participants, rather than meanings I might have ascribed to occurrences if divorced from those perspectives.

Last, the third limitation involves observer effects. Also referred to as reactivity, observer effects occur when the presence of the researcher influences participant behavior (Maxwell, 2006). Some have suggested that observer effects bias or invalidate qualitative findings (LeCompte and Goetz, 1982) or prevent researchers from accurately documenting the social phenomenon of study (Agar, 1980) based on the assumption that people behave differently when they know they are being studied. According to this view, observer effects are uniformly undesirable because they contaminate the setting of the study (Hunt, 1985).

Monahan and Fisher (2010) have taken an opposing view of observer effects, arguing that "informants" performances - however staged or influenced by the observer often reveal profound truths about social and/or cultural phenomena” (p. 358). They contend that researcher presence may not cause participants to self-censor and that, when they occur, participants" "staged performances" (p. 358) can yield rich data by 
communicating their hopes, ideals, values, perceptions of the researcher, and information about how they would like to be seen.

In the present study, the possibility existed that observer effects led participants to (a) be overly cooperative by saying what they believed I wanted to hear, (b) be less candid by not saying what they believed I did not want to hear, (c) think about differentiated instruction in greater depth or with greater frequency than if they had not been in the study, or (d) enact practices of differentiation in ways that they would not have if they had not been in the study. I subscribe to a view of observer effects as offering potential benefits. While I worked to minimize observer effects, I did not keep such a distance from participants that it restricted my access to data by detracting from my rapport with them. My focus was on creating an open environment for honest dialogue with participants in which I communicated that my presence was nonevaluative. I ensured that all interviews were conducted in private spaces that protected confidentiality and carefully structured questions to communicate my non-evaluative stance. I frequently considered my researcher role and observer effects in reflexive journaling, and wrote analytic memos about this issue throughout data collection and analysis.

Karen stated directly during a member checking exercise that she did not believe her teacher behavior in the fall had been affected by her participation in the study (interview, 12/08/14). However, as discussed above in the Study Participation subsection of Chapter Five, Nicole's Phase 2 comments suggested she was keenly aware of being a study participant (interview, 11/14/14, 12/09/14). She characterized her participation in the study as an "incentive" to differentiate, as causing the topic of differentiation to 
remain at the forefront of her thinking throughout the fall, and as a major support for her implementation of differentiation (Nicole, interview, 12/09/14). Nevertheless, she stated during a member checking exercise that she believed her implementation of differentiation would not have looked any different during her first year in the classroom if she had not been in the study (interview, 12/09/14). Nicole concluded by saying that, overall, she was unsure how her participation in the study may have influenced her (interview, 12/09/14). The relationships among Nicole's participation in the study, her conceptions of differentiation, and her practice are complex. Her participation kept the topic of differentiation on her mind during the fall, but she did not think her practice would look any different if she had not participated in the study.

It seems likely that being interviewed regularly about differentiation's role in their own classroom would inevitably cause any participant to think about the model in more depth or with greater frequency than if she did not participate in such interviews. Although being observed would likely also affect teaching behavior in at least some minor ways, study participation is by no means a guarantee of meaningful changes in behavior in terms of implementing differentiation differently in classroom practice. This may be particularly true if (a) participants have an existing, long-term relationship with the researcher, (b) participants are observed on multiple occasions for long periods of time, and (c) participants feel the researcher's presence to be non-evaluative and nonthreatening. In the present study, I had a long-term working relationship with the participants, and I observed them on multiple occasions for up to an hour and a half. Additionally, Karen and Nicole both volunteered that they found my presence non- 
evaluative and non-threatening without my asking them specifically about this issue (Karen, interview, 12/08/14; Nicole, interview, 12/09/14).

At Newland Academy and Lambert Elementary, Karen and Nicole were expected to differentiate, and Nicole was formally evaluated on its execution. Modification of language arts and math instruction by readiness was the norm in both these settings, and both schools trained their teachers in the use of the Daily 5 structure for independent work to support its implementation. Neither participant modified instruction by interest or learning profile in the fall, two approaches to instruction that were not required by their schools. Additionally, I used data source triangulation to determine that Beth's description of Karen's regular practice (Beth, interview, 12/11/14) and Sharon's description of Nicole's regular practice (Sharon, interview, 10/20/14) fully aligned with the practice I observed in their classrooms and which the participants described during interviews. I could find no evidence in the data corpus to suggest that the participants' instructional practice was significantly different when they were not being observed for the study. This appears to support Nicole's contention that her practice would not look significantly different if she were not in the study. However, it seems evident that Nicole's participation did influence her thinking about differentiation. Rather than biasing the research findings, I view the relationships among Nicole's participation in the study, her conceptions of differentiation, and her practice as revealing data about her aspirations for and beliefs about her teaching that allowed me to better understand the phenomena of study. 


\section{Implications}

The purpose of this multicase study was to examine how novice teachers made meaning of Tomlinson's $(1999,2014)$ model of differentiated instruction across preservice coursework specifically focused on the model and their early teaching careers. Its ultimate goal was to inform the field of teacher education about preparing preservice teachers to respond to diverse student needs in their future classrooms through differentiated instruction. This section therefore addresses four principal implications for teacher educators in light of the assertions presented above and suggestions for future teacher education research.

Many teacher education programs in the United States and internationally do not provide preservice teachers with the knowledge, skills, and dispositions necessary to teach students with diverse learning needs in the general education classroom successfully (e.g., Santangelo \& Tomlinson, 2012; Wertheim \& Leyser, 2002). Increasing attention has been devoted to this problem as educational experts and professional organizations continue to call for general education teachers to design curriculum and instruction to better fit the needs of individual learners (e.g., National Association for the Education of Young Children, 2003; National Council for Teachers of English \& The International Reading Association, 2000; National Council of Teachers of Mathematics, 2006; Siegel \& Shaughnessy, 1994).

Some of the implications below are offered as recommendations for teacher education programs that respond to this call by seeking to prepare preservice teachers to effectively address academic diversity in their future classrooms through differentiation. However, other implications that can be drawn from the study apply more broadly to 
teacher education programs with a variety of core missions and values, especially those which prepare teacher candidates to enact varied ambitious practices. Thus, for each identified implication, I discuss its potential applicability both to any teacher education program and to programs or courses that emphasize preparing teacher candidates to differentiate.

\section{Implication 1: Teacher education programs should ensure that coursework for} preservice teachers is founded upon a unified conceptual framework of teaching and learning reflected in the shared knowledge and beliefs of instructors.

Having a coherent conception of teaching and learning that undergirds preservice experiences is paramount for supporting students in making connections among ideas across courses. How students draw these connections can be enhanced or inhibited by the design of a teacher education program. When program design inhibits connectionmaking, it may be an indication of conceptual and structural fragmentation (Zeichner \& Gore, 1990), which frequently arises when teacher education courses do not reflect a common philosophy of teaching and learning (Darling-Hammond \& Hammerness, 2007). According to Darling-Hammond and Hammerness (2007), programs with a coherent vision are "founded on a set of big ideas" (p. 392) and "shared knowledge and beliefs about teaching and learning" (p. 392) by faculty members. The coherence offered by a shared conceptual framework "provides a guiding vision of the kind of teacher the program is trying to prepare" (Feiman-Nemser, 2001, p. 1023), a vision that is based on values that are core to its mission. Faculty must believe in these values and then put them into practice through their curriculum and instruction reflecting aligned conceptualizations of key topics and ideas. Programs with a coherent conception of 
teaching and learning have been found to exert greater influence over the conceptions and practice of preservice teachers than programs with courses that are disconnected (Darling-Hammond \& Hammerness, 2007).

Implications for programs focused on differentiation. In a teacher education program that considers preparing preservice teachers to differentiate instruction as one of its chief goals, the cornerstone of such a conceptual framework would be foundational understandings about the importance and nature of academic diversity and responses to it. Preservice teachers must come to own these core ideas for themselves if they are to transfer the ideas into eventual classroom practice (Sherman, 2009). To achieve this, the content, processes, and products with which preservice teachers work should promote deepening understanding of the needs of academically diverse learners (Tomlinson et al., 1997). The program must communicate implicitly and explicitly that differentiation is both a professional and ethical responsibility. Tomlinson et al. (1994) suggest that if:

Preservice teachers sense that differentiating instruction for academically diverse learners is a low priority for their teacher education institutions, cooperating teachers, and university supervisors...rather than [their teacher education experiences] being a time of internship or residency during which special diagnostic and prescriptive skills will be developed for addressing needs of academically diverse learners, preservice teachers will gain tacit permission to dispense learning as though all students need the same prescription or treatment. (p. 113)

A conceptual framework reflecting the principles of differentiated instruction demonstrates an understanding that the model encompasses a philosophy of education. To differentiate effectively, preservice teachers must also come to develop this understanding. They must draw a distinction between the underlying principles and the strategies or organizational structures of differentiation, as knowledge of strategies alone cannot sustain robust implementation of the model, and this distinction should be 
strongly emphasized in teacher education coursework (Sherman, 2009). As Sherman (2009) explains:

Teacher candidates are usually eager to learn the 'how to' in methods classes to gain confidence and feel competent to teach in their own classrooms. But a rapid jump to the "how to" may sacrifice attention to the "why," which provides a rationale for selecting particular teaching techniques and may better support progressive educational practice for the long-term. (p. 51)

The principles of differentiation must guide the use of the practices of differentiation.

Otherwise, differentiation may become another discrete strategy to be added on to regular practice occasionally, rather than a lens through which to view teaching and learning. Making the "why" of differentiation a primary focus of teacher education coursework is therefore necessary.

Because the effective implementation of a unified conceptual framework reflecting the principles of differentiation requires shared knowledge of the model among teacher educators, a significant problem exists when some instructors of preservice teachers lack this knowledge. This may be the result of instructors having limited experience teaching in diverse K-12 classrooms or lacking exposure to particular principles and practices of the model. In this instance, faculty as a group and individually must make strategic decisions about gaining knowledge of how teachers respond to diversity effectively in contemporary public school classrooms. This might involve seeking assistance from teacher educators with expertise in differentiation within or beyond the program to facilitate meaningful dialogue about the nature and role of differentiation in K-12 settings, as well as in preservice teacher education coursework. It is important to note that this support for faculty should likely be differentiated. This might be accomplished by allowing faculty to identify principles or practices about which 
they feel they would benefit from gaining additional knowledge and to choose the format through which they would prefer to receive this information.

\section{Implication 2: Teacher education programs should ensure that best practices in instruction are modeled often to support the development of images of their robust implementation.}

Preservice teachers may see best practices enacted by educators in two contexts. They may be modeled by CIs in field placements and by instructors in teacher education coursework. Teacher educators generally have more control over the latter context than the former. In the context of teacher education, modeling takes on a unique role because teacher educators are in the business of teaching how to teach. In this way, a teacher educator's instructional practice is an especially powerful force. The likelihood that preservice teachers will be able to effectively translate course content into their future practice increases when instructors in teacher education programs model practices and beliefs (Grossman et al., 2000; Struyven, Dochy, \& Janssens, 2008; Utley, 2006). Field placements are also powerful settings in which preservice teachers develop knowledge and beliefs about what they are learning from coursework to be best practices in instruction, including ambitious practices. These settings can provide teacher candidates with images of what the effective implementation of these practices looks like. Of course, they can provide images of what ineffective or lack of implementation looks like as well. Prompting preservice teachers to reflect on and share what they are seeing in field placements as it relates to best practices with instructors or university supervisors is crucial. 
It is critical to note that preservice teachers may not be aware of every instance and aspect of best practices modeled for them in field placements or university coursework. Learning to teach is a developmental journey spanning many years and stages, and preservice teachers may not yet have facility with fully recognizing observed examples of particular instructional practices or approaches when they are not explicitly identified as such. Teacher candidates are still students and may have difficulty adopting the lens or mindset of a teacher to analyze the finer points of instructional decisionmaking. Thus, just because preservice teachers report that they do not recall seeing a practice modeled does not mean that they have not seen or experienced it. This suggests that teacher educators should devote significant time to debriefing with preservice teachers about the use of best practices in coursework, and that preservice teachers should be prompted to look for more subtle or unexpected forms of particular practices, approaches, or techniques.

Implications for programs focused on differentiation. Every effort should be made to identify field placements for preservice teachers that would help them develop images of what differentiated instruction looks like in a K-12 setting. Interacting with CIs who believe, practice, and articulate their understanding of differentiation can be highly supportive of novice teachers' conceptions and practice (Tomlinson et al., 1997). Such field placements bridge the gap between theoretical coursework and the realities of their future classroom practice (Sherman, 2009). Filmed examples of high quality teaching episodes illustrating key principles and practices of differentiation might also supplement this image development. If finding such settings is not possible for preservice teachers' early fieldwork, they might be encouraged to observe specific 
students in their placement and keep a reflective journal in which they consider (a) whether those students' learning needs are being met, particularly during whole-class instruction, and (b) how learning experiences they observed might have been restructured to better meet the students' needs (Sherman, 2009). These reflections might be shared and discussed in class meetings, providing teacher candidates with opportunities to brainstorm ideas or ask questions about the varied ways in which student readiness, interest, learning profile, or affective needs might be addressed in the particular contexts presented by their field placements. Preservice teachers should also be prompted to look for structures or strategies in the classroom that could be used to support differentiation, even if the CI does not refer to them explicitly as differentiated techniques or subscribe to the larger philosophy of differentiated instruction, such as the Daily 5 and Daily 3 frameworks. Teacher candidates may be asked to consider how such structures, for which they can develop a robust image of implementation based on modeling they observe, might be used in similar or different ways to support differentiated instruction. During student teaching, university programs may require preservice teachers to plan differentiated lessons. These requirements may ask teacher candidates to develop lessons that feature high quality curriculum and that use ongoing assessment data to inform instruction. Preservice teachers might be required to design several lessons that modify instruction based on readiness and be encouraged to design other lessons that respond to interest or learning profile. They might be asked to complete brief but meaningful reflections first as part of the lesson planning process and then after they have presented the lesson. Sherman (2009) suggested that posing specific questions for teacher candidates to answer about their lesson plans could prompt them to articulate how 
their plans address the needs of students in their class. She offered the question "What is the potential in the lesson for every student to be highly involved in the learning process?" (p. 52) as an example of prompting students to consider how the principles of student engagement in learning are reflected in their lesson. Other questions might ask about how the lesson reflects the principles of high quality curriculum; how specific assessment data is used to inform lesson design, group students, or create different versions of tasks; and how effectively learning activities respond to identified student differences. These reflection questions might also prompt preservice teachers to explain how their differentiated lessons reflect the principle of "teaching up" (Tomlinson \& Javius, 2012) as a manifestation of a growth mindset.

The university supervisor can play a key role in supporting preservice teachers as they work to design and execute lessons addressing diverse student learning needs and consider the degree of alignment between their field placements and coursework. As mentors, they can provide examples of what putting the principles of differentiation into action in a particular classroom context might look like, provide individualized feedback on lesson plans, and discuss any issues of misalignment between the conceptions of teaching and learning in a placement and the teacher education program. The same is true of instructors leading seminars for preservice teachers during student teaching experiences.

Additionally, instructors of teacher education courses should provide unambiguous models of the practices of differentiated instruction in action and related beliefs. Modeling should demonstrate fidelity to the full model of differentiation and should emphasize the interdependence of curriculum, assessment, instruction, and 
learning environment (Santangelo \& Tomlinson, 2012). Instructors should devote significant time to explicitly discussing varied aspects of their differentiated practice with their students, rather than assuming that each instance of their modeling the model has been noted and considered by students. Modeling differentiation in teacher education coursework better allows instructors to meet the learning needs of preservice teachers, supporting teacher candidate pools reflecting increasingly diverse backgrounds and experiences (Santangelo \& Tomlinson, 2012). Such an approach would give preservice teachers a voice for sharing their needs and their perceptions of instructional fit (Santangelo \& Tomlinson, 2012).

\section{Implication 3: Teacher education programs should incorporate repeated, thought-} provoking opportunities for preservice teachers to examine their beliefs about teaching and learning, including "mindset" (Dweck, 2007), in a variety of contexts throughout their course of study.

Reflection is a skill central to effective teaching practice. Teacher education programs must support preservice teachers in developing reflective habits about both the technical and ethical aspects of their work. Teaching can be an intensely personal endeavor. The prior beliefs about teaching and learning preservice teachers bring to teacher education programs may serve as a filter through which they evaluate, accept, or discard new ideas they encounter. In their large-scale review of empirical literature on teacher education programs to date, Wideen et al. (1998) noted that studied programs with more productive approaches had students examine their prior beliefs and then build upon them. Preservice teachers must be provided with safe spaces in which they can 
make such candid assessments. According to Borko and Putnam (1996), teacher education programs

must challenge participants' beliefs about teaching, learning, subject matter, self as teacher, and learning to teach. They must help prospective teachers make their implicit beliefs explicit and create opportunities for them to confront the potential inadequacy of those beliefs. They should also provide opportunities for prospective teachers to examine, elaborate, and integrate new information into their existing systems of knowledge and belief. (p. 701)

In particular, preservice teachers should learn about implicit theories of intelligence, or "mindset" (Dweck, 2007). As they carefully examine their own mindsets, teacher candidates should consider how a teacher conveys beliefs about intelligence to students and the potential effects of those messages on students. Prospective teachers should also consider how students' mindsets may affect their academic performance and attitude toward learning. Likewise, teacher educators should consider how they communicate their own beliefs about intelligence to preservice teachers and whether they may be promoting a fixed or growth mindset in them. These messages may be subtly communicated through indirect comments or course materials (Jones, Bryant, Snyder, \& Malone, 2012). Teacher educators might share research findings about the influence of teacher mindset on students, or of student mindset on academic performance, with teacher candidates as prompts for reflection.

Implications for programs focused on differentiation. In the case of a teacher education program in which responding to the needs of all learners is a key component of its conceptual framework of teaching and learning, preservice teachers must be given repeated opportunities to integrate new information on this topic into their existing belief system. In particular, teacher candidates must be prompted to honestly examine their deep beliefs about diverse learners (Stodolsky \& Grossman, 2000; Tomlinson et al., 
1995). They must actively consider the connections among those beliefs and their knowledge, skills, and dispositions surrounding approaches to identifying and responding to student needs (Goodnough, 2010). It is unlikely that the principles of differentiated instruction will be put into practice in the classroom of a teacher who does not believe all students have the capacity to grow beyond where they began and is not deeply committed to meeting the needs of all learners. Preservice teachers should therefore be asked to consider the ways in which teaching is an ethics-based profession and differentiation's potential role in the ethics of teaching diverse students to support their success. As noted above, preservice teachers might be asked to reflect on the relationship between a growth mindset and specific practices such as teaching up and flexible grouping or the broad philosophy of differentiation.

Another important area of reflection for teacher candidates involves recognizing the presence or lack of differentiation in the world around them and developing connections between that recognition and their practice. From their past academic experiences at K-12 or university levels, they may identify powerful examples of differentiation or may see a significant need where it was absent. Likewise, they may note instances in which its implementation would have been beneficial or even critical for the academic success of a family member or close friend. They may also have seen principles of differentiation at work in settings outside of the classroom, such as in experiences with parenting or coaching. Recognizing the presence or absence of differentiation in their own experiences may influence their beliefs in its importance or feasibility and may offer new perspectives on its role in their practice. 


\section{Implication 4: Teacher education programs should guide preservice teachers in} developing skills to respond to the complex demands of early teaching, especially in settings in which best practices of instruction as presented in teacher education programs are not fully supported.

Teacher educators cannot predict the types of settings in which graduates of their program will be employed or prepare preservice teachers for every possible contingency; however, programs can help students develop general skills that will assist them in responding to challenges as they begin to teach in varied contexts. These skills may be addressed in coursework or student teaching seminars, and they may be reinforced by the university supervisor during student teaching. At the core of these skills is a need for deep reflection, which does not end when teachers graduate from preservice programs.

On the contrary, developing reflective habits of mind as a teacher candidate can and should carry forward into induction experiences and beyond in important ways. Examples of these skills for new teachers involve seeking help, handling feelings of conflict, and assessing fit between teaching context and personal philosophy of teaching. Below, for each of these examples, I provide questions related to that skill that a new teacher might ask herself. These questions illustrate a new teacher's personal analysis and problem-solving processes. Teacher educators might share similar questions with preservice teachers and discuss a variety of responses.

The first skill involves seeking help, which will likely occur in every teaching context including those highly supportive of best practices in instruction. A new teacher may ask:

- How do I identify and define a question I have or a problem I am experiencing? 
- Whom should I ask for help?

- At what stage in this problem's development should I ask for help?

- What may happen if I wait to ask for help?

It is likely that numerous graduates of teacher education programs will be enculturated into communities of practice in which some or many of the best practices they learned in teacher education programs are not enacted. In such a setting, new teachers may experience feelings of conflict. To handle these feelings productively, a novice may ask:

- What am I feeling?

- What is the source of this feeling?

- Whom can I look to for support in dealing with this feeling?

- What is within my power to change that might reduce or eliminate this feeling?

- How can I continue to teach effectively in the face of this feeling?

In some instances, these feelings of conflict may be so strong that a teacher feels compelled to assess the fit between her current teaching context and her personal philosophy of teaching. In this case, a beginning teacher might ask:

- What are the values of this community of practice?

- How do those values align with my values, and how do they differ?

- Is this the right teaching environment for me?

- Is there anything in this teaching environment that could change which would make it the right setting for me? Who has the power to make that change?

- Do I know of another setting that would be better for me, or can I make this one work? 
Bianchini and Brenner (2009) have also suggested that teacher educators prepare their students for this situation by providing them with specific strategies for challenging existing practices with their peers. Such an approach should be used judiciously. Beginning teachers typically assume peripheral roles when they enter communities of practice and move toward more central roles with time and experience (Wenger, 1998). Novices must distinguish between productive professional discussions with peers and administrators about their knowledge, beliefs, and desires, and approaches that may significantly alienate experienced colleagues or jeopardize their employment.

Implications for programs focused on differentiation. The findings of the present study suggest that, to encourage the implementation of differentiated instruction, new teachers may benefit from a community of practice in which (a) their colleagues share their beliefs and knowledge about differentiation, and put those beliefs and knowledge into practice; (b) they receive structured, deep support of their differentiated practice; and (c) they have the flexibility to respond to student needs.

Not all beginning teachers will join communities of practice in which these elements are present or well-developed. On the contrary, in light of the continued prevalence of one-size-fits-all instruction (Brighton et al., 2005; Kerry \& Kerry, 1997; Moon, Tomlinson, \& Callahan, 1995; Schumm et al.,1995; Stodolsky \& Grossman, 2000), it is likely that many graduates of teacher education programs will join communities of practice characterized by standardization and uniformity that are not responsive to diverse learner needs (Tomlinson et al., 1997). According to Tomlinson and colleagues (1997), in these instances, the "pull" to teach in ways that align to local practice norms can be "overwhelming" (p. 276) for new teachers, despite their strong 
beliefs in the importance of responsive instruction. In such an environment in which “differentiation is an oxymoron" (Tomlinson et al., 1997, p. 277), or in an environment that is not quite as inhospitable to differentiation but that does not offer adequate support or flexibility, new teachers may experience acute conflict. Teacher educators should help preservice teachers recognize that, like all aspects of the long learning to teach trajectory, conceptualizing and implementing differentiation is an ongoing process, and they should continue to reflect on their growth in this process in relation to their teaching setting. Instructors should explicitly guide preservice teachers in considering how they might pose and answer the questions above when seeking help, handling feelings of conflict, and assessing fit with their personal philosophy if they find themselves in a future teaching context that does not feature a shared vision of differentiation, deep support of differentiated practice, or the flexibility to respond to student needs. Teacher educators might also encourage teacher candidates to consider opportunities for professional development on differentiation in their future careers, including opportunities provided by their school or district and those which teachers might seek out on their own.

\section{Future Research}

This study suggests several avenues for future research related to how novice teachers make meaning of differentiated instruction through study and through teaching practice. Before the present study, no identified empirical research had examined how preservice teachers make meaning of Tomlinson's model of differentiated instruction in a teacher preparation course in which differentiation serves as the primary focus of instruction. Further, no identified studies had examined how novice teachers make meaning of differentiation across both a teacher preparation course on differentiation and 
experiences in their early teaching careers. While this study takes a first step toward addressing this significant gap in the literature, far more research is needed to develop a nuanced understanding of these phenomena across varied teacher education and school of employment settings and among diverse novice teachers at elementary and secondary levels.

Future research might examine the efficacy of varied methods of teaching differentiated instruction, including modeling it, in terms of the development of preservice teachers' conceptions of the model in the short term during teacher education programs and over the long term once they enter the classroom. It might examine teacher education programs that effectively prepare instructors, university supervisors, and cooperating teachers to mentor student teachers in responding to academic diversity to determine how those programs communicate a consistent message and support novices. Future empirical study might also explore in greater depth the roles of contextual factors that support or challenge the implementation of differentiation in K-12 classrooms and the common features of communities of practice that are highly supportive of implementation. In particular, the contention that the three pillars of a shared vision, structured support, and the flexibility to respond to student needs may be core elements of contexts that encourage implementation might be investigated in other settings.

Last, this study's findings suggested a significant relationship may exist between teacher mindset (Dweck, 2007) and attitudes toward responding to academic diversity. These attitudes include the willingness to develop the skills and dispositions needed for effective differentiation and to put them into practice despite the complex demands of early teaching. More research on the nature and effect of this potential relationship is 
needed, as well as research on broader issues involving how teacher mindset may influence K-12 students, how teacher educator mindset may influence preservice teachers, and how the beliefs of preservice teachers with fixed mindsets might be changed, if at all, toward a growth orientation.

\section{Conclusion}

Preservice teachers face a formidable challenge. They must come to know content and pedagogy. They must gain skill in planning and management. They must scrutinize and question long-held beliefs. They must become equally prepared to work with a child who is five or 11 . And, in teacher education programs that value it, they must gain the knowledge, skills, and beliefs to meet the needs of academically diverse learners effectively. Differentiating instruction is complex. Because it responds to individual learners, it can have no recipe or standardized practice, and it requires intellectually rigorous, time-intensive work (Sherman, 2009). This may cause some to suggest that it is unreasonable to expect novice teachers to differentiate instruction, and that teaching preservice teachers about the model in depth is therefore a misuse of the limited time teacher candidates spend in their programs. Tomlinson et al. (1995) explain:

Given the complexities of teaching, the difficulties novices have making sense of classroom events, and the amount of experience needed to develop competence in the classroom, it might seem unrealistic to expect preservice teachers to provide differentiated instruction. On the other hand, patterns of teaching that form early in a career may become entrenched and thus never change...[I]t can be argued that introducing novices to student-centered views of instruction and giving them practice in implementing strategies may be necessary to break the one-size-fits-all conception of teaching....Early teaching is a time to develop the "gross motor skills" of the profession. Robust differentiation is a "fine motor skill" of teaching. Thus, few novice teachers will display great proficiency in planning and facilitating fully differentiated classrooms, and they should not be expected to do so. Nonetheless, there is a need to help novices develop the "gross motor skills" that ultimately will evolve into the "fine motor skills" of responsive teaching. (pp. $\mathrm{x}-\mathrm{xi}$ ) 
The findings of the present study support this contention: Providing preservice teachers with in-depth opportunities to make meaning of the philosophy, principles, and practices of differentiation appeared to reinforce the importance they ascribed to meeting the needs of academically diverse students and provide them with skills for responsive instruction. While they do not yet facilitate fully differentiated classrooms, Karen and Nicole appear to have well-developed "gross motor skills." Because inservice teachers tend to move closer to developing "fine motor skills" and to implement more of the practices they learned about in teacher education contexts during their second year in the classroom (Grossman et al. 2000; Marbach-Ad \& McGinnis, 2009; Steele, 2001), I plan to continue to study the participants' conceptions of differentiated instruction and related practice into 2015-2016. 


\section{References}

Adams, P. E., \& Krockover, G. H. (1997). Beginning science teacher cognition and its origins in the preservice science teacher program. Journal of Research in Science Teaching, 24, 633-653.

Adams, T. L. (1998). Prospective elementary teacher's mathematics subject matter knowledge: The real number system. Journal for Research in Mathematics Education, 20, 35-48.

Adler, J., \& Reed, Y. (Eds.). (2002). Challenges of teacher development: An investigation of take-up in South Africa. Pretoria, South Africa: Van Schaik.

Agar, M. (1980). Getting better quality stuff: Methodological competition in an interdisciplinary niche. Urban Life, 9(1), 34-50.

Alliance for Excellent Education. (2004). Tapping the potential: Retaining and developing high-quality new teachers. Retrieved on April 6, 2014 from http://chalkboardproject.org/wp-content/uploads/2011/01/ment-15.pdf

Anderson, N. H. (1971). Integration theory and attitude change. Psychological Review, 78(3), 171-206.

Ball, D. L., (1988). Research on teacher learning: Studying how teachers' knowledge changes. Action in Teacher Education, 10(2), 17-23. 
Ball, D. L. (1989). Breaking with experience in learning to teach mathematics: The role of a preservice methods course. Paper presented at the annual meeting of the American Educational Research Association, San Francisco.

Ball, D. L. (1990a). The mathematical understandings that prospective teachers bring to teacher education. Elementary School Journal, 90, 449-466.

Ball, D. L. (1990b). Prospective elementary and secondary teachers' understanding of division. Journal of Research in Mathematics Education, 21, 132-144.

Ballou, D., \& Podgursky, M. (2000). Reforming teacher preparation and licensing: What is the evidence? Teachers College Record, 102(1), 1-27.

Bandura, M., \& Dweck, C. S. (1985). The relationship of conceptions of intelligence and achievement goals to achievement-related cognition, affect, and behavior. Unpublished manuscript, Harvard University.

Bartholomew, J. (1976). Schooling teachers: The myth of the liberal college. In G. Whitty \& M. Young (Eds.), Explorations in the politics of school knowledge (pp. 114-124). Drifferton, England: Nafferton.

Beecher, M., \& Sweeny, S. M. (2008). Closing the achievement gap with curriculum enrichment and differentiation: One school's story. Journal of Advanced Academics, 19(3), 502-530.

Berliner, D. (2005). Our impoverished view of educational reform. Teacher College Record ID number 12106. Retrieved from http://www.tcrecord.org.

Bianchini, J. A., \& Brenner, M. E. (2009). The role of induction in learning to teach toward equity: A study of beginning science and mathematics teachers. Science Education, 94, 164-195. 
Blackwell, L. S., Trzesniewski, K. H., \& Dweck, C. S. (2007). Implicit theories of intelligence predict achievement across an adolescent transition: A longitudinal study and an intervention. Child Development, 78(1), 246-263.

Blumer, H. (1969). Symbolic interactionism. Berkeley, CA: University of California Press.

Borko, H. (2004). Professional development and teacher learning: Mapping the terrain. Educational Researcher, 33(8), 3-15.

Borko, H., Eisenhart, M., Brown, C. A., Underhill, R. G., Jones, D., \& Agard, P. C. (1992). Learning to teach hard mathematics: Do novice teachers and their instructors give up too easily? Journal for Research in Mathematics Education, 23, 194-222.

Borko, H., Peressini, D., Romagnano, L, Knuth, E., Willis-Yorker, C., Wooley, C., ... Masarik, K. (2000). Teacher education does matter: A situative view of learning to teach secondary mathematics. Educational Psychologist, 35(3), 193-206.

Borko, H., \& Putnam, R. T. (1996). Learning to teach. In D. Berliner \& R. Calfee (Eds.). Handbook of educational psychology (pp. 673-708). New York, NY: MacMillan.

Boushey, G., \& Moser, J. (2006). The Daily 5: Fostering literacy independence in the elementary grades. Portland, ME: Stenhouse.

Boushey, G., \& Moser, J. (2014). The Daily 5: Fostering literacy independence in the elementary grades (2nd ed.). Portland, ME: Stenhouse.

Bransford, J., Brown, A. L., Cocking, R. R., \& National Research Council (U.S.). (1999). How people learn: Brain, mind, experience, and school. Washington, D.C: National Academy Press. 
Brighton, C. M., Hertberg, H. L., Moon, T. R., Tomlinson, C. A., \& Callahan, C. M. (2005). The feasibility of high-end learning in a diverse middle school. RM05210. Storrs, CT: The National Research Center on the Gifted and Talented.

Brouwer, N., \& Korthagen, F. (2005). Can teacher education make a difference? American Educational Research Journal, 42(1), 153-224.

Brown, A. L., Palincsar, A. S., \& Purcell, L. (1984). Poor readers: Teach don’t label. In U. Neisser (Ed.), The academic performance of minority children: A new perspective. Hillsdale, NJ: Erlbaum.

Brown, C. A., \& Borko, H. (1992). Becoming a mathematics teacher. In D. A. Grouws (Ed.), Handbook of research on mathematics teaching and learning (pp. 209239). New York, NY: Macmillan.

Brown, J. S., \& Duguid, P. (1991). Organizational learning and communities-of-practice: Toward a unified view of working, learning, and innovation. Organization Science, 2(1), 40-57.

Bryan, L. A., \& Atwater, M. M. (2002). Teacher beliefs and cultural models: A challenge for science teacher preparation programs. Science Teacher Education, 86, 821839.

Cady, J., Meier, S. L., \& Lubinski, C. A. (2006). Developing mathematics teachers: The transition from preservice to experienced teacher. The Journal of Educational Research, 99(5), 295-305.

Calderhead, J. (1996). Teachers: Beliefs and knowledge. In D. Berliner \& R. Calfee (Eds.). Handbook of educational psychology (pp. 709-725). New York, NY: MacMillan. 
Carroll, T. G. \& Foster, E. (2010). Who will teach? Experience matters. Washington, DC: National Commission on Teaching and America's Future.

Carter, K., \& Doyle, W. (1987). Teachers' knowledge structures and comprehension processes. In J. Calderhead (Ed.), Exploring teachers' thinking (pp. 147-160). London: Cassell.

Cavanagh, M., \& Prescott, A. (2007). Professional experience in learning to teach secondary mathematics: Incorporating pre-service teachers into a community of practice.. In J. Watson \& K. Beswick (Eds.), Mathematics: Essential research, essential practice-Volume 1: Proceedings of the $30^{\text {th }}$ annual conference of the Mathematics Education Research Group of Australia (pp. 182-191). Brisbane, QLD: MERGA.

Center on Education Policy. (2006). A public education primer: Basic (and sometimes surprising) facts about the U.S. education system. Washington, D.C.

Center on Education Policy. (2007). Why we still need public schools: Education for the common good. Washington, D.C.

Center on Education Policy. (2009). How state and federal accountability policies have influenced curriculum and instruction in three states: Common findings from Rhode Island, Illinois, and Washington. Washington, D.C.

Charmaz, K. (2006). Constructing grounded theory: A practical guide through qualitative analysis. Los Angeles, CA: Sage.

Clandinin, D. J. (1986). Classroom practice: Teacher images in action. Philadelphia, P.A.: Falmer Press. 
Coburn, C. E., \& Russell, J. (2008). District policy and teachers'social networks. Educational Evaluation and Policy Analysis, 30(3), 203-235.

Coffey, A., \& Atkinson, P. (1996). Making sense of qualitative data: Complementary research strategies. Thousand Oaks, CA: Sage.

Creswell, J. W. (2013). Qualitative inquiry and research design: Choosing among five approaches ( $3^{\text {rd }}$ ed.). Thousand Oaks, CA: Sage.

Darling-Hammond, L. (2000a). Reforming teacher preparation and licensing: Debating the evidence. Teachers College Record, 102(1), 28-56.

Darling-Hammond, L. (2000b). Teacher quality and student achievement: A review of state policy evidence. Education Policy Analysis Archives, 8. Retrieved from http://epaa.asu.edu/v8n1/2000.

Darling-Hammond, L. (2006). Constructing twenty-first century teacher education. Journal of Teacher Education, 57(3), 300-314.

Darling-Hammond, L., \& Hammerness, K. (2007). The design of teacher education programs. In L. Darling-Hammond \& J. Bransford (Eds.), Preparing teachers for a changing world: What teachers should learn and be able to do (pp. 390-441). San Francisco, CA: Jossey-Bass.

Darling-Hammond, L., \& Youngs, P. (2002). Defining "highly qualified teachers:” What does "scientifically-based research" actually teach us? Educational Researcher, 31(9): 13-25.

Dee, A. L. (2011). Preservice teacher application of differentiated instruction. The Teacher Educator, 46, 53-70. 
Diener, C. I., \& Dweck, C. S. (1978). An analysis of learned helplessness: Continuous changes in performance, strategy, and achievement cognitions following failure. Journal of Personality and Social Psychology, 36, 451-462.

Diener, C. I., \& Dweck, C. S. (1980). An analysis of learned helplessness: (II) The processing of success. Journal of Personality and Social Psychology, 39, 940952.

Diller, D. (2003). Literacy work stations: Making centers work. Portland, ME: Stenhouse.

Dweck, C. S. (1975). The role of expectations and attributions in the alleviation of learned helplessness. Journal of Personality and Social Psychology, 31, 674-685.

Dweck, C. S. (2000). Self-theories: Their role in motivation, personality, and development. New York: Psychology Press.

Dweck, C. S. (2007). Mindset: The new psychology of success. New York: Ballantine.

Dweck, C. S. \& Leggett, E. L. (1988). A socio-cognitive approach to motivation and personality. Psychological Review, 95, 256-273.

Edwards, R. (1979). Contested terrain: The transformation of the workplace in the $20^{\text {th }}$ Century. New York, NY: Basic.

Elksmin, L. K. (2001). Implementing case method of instruction in special education teacher preparation programs. Teacher Education and Special Education, 24, 95107.

Ensor, P. (2001). From preservice mathematics teacher education to beginning teaching: A study in recontextualizing. Journal for Research in Mathematics Education, 32(3), 296-320. 
Erickson, F. (1986). Qualitative methods in research on teaching. In M. C. Wittock (Ed.), Handbook of research on teaching (pp. 119-161). New York, NY: Macmillan.

Feiman-Nemser, S. (2001). From preparation to practice: Designing a continuum to strengthen and sustain teaching. Teachers College Record, 103(6), 1013-1055.

Feiman-Nemser, S., \& Buchmann, M. (1983). Pitfalls of Experience in Teacher

Preparation. East Lansing, MI: Institute for Research on Teaching, Michigan State University.

Feiman-Nemser, S., \& Buchmann, M. (1985). The First Year of Teacher Preparation: Transition to Pedagogical Thinking? Research Series No. 156. East Lansing, MI: Institute for Research on Teaching, Michigan State University.

Feiman-Nemser, S., \& Buchmann, M. (1986). The first year of teacher preparation: Transition to pedagogical thinking? Journal of Curriculum Studies, 18(3), 239256.

Felter, M. (1999). High school staff characteristics and mathematics test results. Education Policy Analysis Archives, 7. Retrieved from http://epaa.asu.edu/epaa/v7n9.html

Fenstermacher, G. D. (1994). The knower and the known in teacher knowledge research. In L. Darling-Hammond (Ed.), Review of research in education (Vol. 20, pp. 356). Washington, D.C.: American Educational Research Association.

Ferguson, P., \& Womack, S. T. (1993). The impact of subject matter and education coursework on teaching performance. Journal of Teacher Education, 44, 55-63.

Flores, M. A. (2006). Being a novice teacher in two different settings: Struggles, continuities, and discontinuities. Teachers College Record, 108(10), 2021-2052. 
Frank, K. A. (1998). Quantitative methods for studying social context in multi-levels and through interpersonal relations. Review of Research in Education, 23, 171-216.

Frank, K. A., Muller, C., Schiller, K., Crosnoe, R., Riegle-Crumb, C., Strassman-Muller, A, \& Pearson, J. (2008). The social dynamics of mathematics coursetaking in high school. American Journal of Sociology, 113, 1645-1696.

Frank, K. A., Zhao, Y., \& Borman, K. (2004). Social capital and the diffusion of innovations within organizations: Application to the implementation of computer technology in schools. Sociology of Education, 77, 148-171.

Frank, K. A., Zhao, Y., Penuel, W. R., Ellefson, N., \& Porter, S. (2011). Focus, fiddle and friends: Experiences that transform knowledge for the implementation of innovations. Sociology of Education, 84(2), 137-156.

Fuller, F., \& Brown, O. (1975). Becoming a teacher. In K. Ryan (Ed.), Teacher education: Seventy-fourth yearbook of the National Society for the Study of Education. Chicago, IL: University of Chicago Press.

Geertz, C. (1973). Thick description: Toward an interpretive theory of culture. In C. Geertz (Ed.), The interpretation of cultures (pp. 3-30). New York, NY: Basic.

Geisler, J., Hessler, R., Gardner, R., \& Lovelace, T. (2009). Differentiated writing interventions for high-achieving urban African American elementary students. Journal of Advanced Academics, 20, 214-247.

Gess-Newsome, J., \& Lederman, N. G. (1993). Preservice biology teachers' knowledge structures as a function of professional teacher education: A year-long assessment. Science Education, 77(1), 25-45. 
Glasser, B. G, \& Strauss, A. L. (1967). The discovery of grounded theory. Chicago, IL: Aldine.

Glickman, C., \& Bey, T. (1990). Supervision. In W. R. Houston (Ed.), Handboook of research on teacher education (pp. 549-566). New York, NY: Macmillan.

Goldhaber, D. D., \& Brewer, D. J. (2000). Does teacher certification matter? High school teacher certification status and student achievement. Educational Evaluation and Policy Analysis, 22, 129-145.

Goodnough, K. (2010). Investigating pre-service science teachers' developing professional knowledge through the lens of differentiated instruction. Research in Science Education, 40, 239-265.

Greeno, J. G. (1997). On claims that answer the wrong questions. Educational Researcher, 26(1), 5-17.

Greeno, J. G. (2003). Situative research relevant to standards for school mathematics. In J. Kilpatrick, W. G. Martin, \& D. Schifter (Eds.). A research companion to Principles and Standards for School Mathematics (pp. 304-332). Reston, VA: National Council of Teachers of Mathematics.

Greeno, J. G., Collins, A. M., \& Resnick, L. B. (1996). Cognition and learning. In D. Berliner \& R. Calfee (Eds.), Handbook of educational psychology (pp. 15-46) New York: NY, Macmillan.

Griffin, M. L., \& Warden, M. R. (2006). The effects of a university public school partnership on the collaborative skills of preservice teachers. International Journal of Learning, 13(5), 187-194. 
Grossman, P. L. (1989a). A study in contrast: Sources of pedagogical content knowledge for secondary English. Journal of Teacher Education, 40(5), 24-31.

Grossman, P. L. (1989b). Learning to teach without teacher education. Teachers College Record, 91, 191-207.

Grossman, P. L. (1990). The making of a teacher: Teacher knowledge and teacher education. New York, N.Y.: Teachers College Press.

Grossman, P. L., Valencia, S. W., Evans, K., Thompson, C., Martin, S., \& Place, N. (2000). Transitions into teaching: Learning to teach writing in teacher education and beyond. Journal of Literacy Research, 32, 631-662.

Guba, E. G., \& Lincoln, Y. S. (1981). Effective evaluation. San Francisco, CA: JosseyBass.

Guyton, E., \& Farokhi, E. (1987). Relationships among academic performance, basic skills, subject matter knowledge, and teaching skills of teacher education graduates. Journal of Teacher Education, 38, 37-42.

Hatala, J., \& Fleming, P. (2007). Making transfer climate visible: Utilizing social network analysis to facilitate transfer of learning. Human Resource Development Review, 6(1), 33-63.

Hawk, P. P., Coble, C. R., \& Swanson, M. (1985). Certification: It does matter. Journal of Teacher Education, 36(3), 13-15.

Hellman, D. W. (2007). Implementing differentiated instruction in urban, Title I schools: Effects of facilitated support groups and program fidelity on student achievement. University of South Florida: Proquest Dissertations and Theses. 
Holland, D., Lachiotte, W., Skinner, D., \& Cain, C. (1998). Identity and agency in cultural worlds. Cambridge, MA: Harvard University Press.

Holloway, J. H. (2000). Preparing teachers for differentiated instruction. Educational Leadership, 58(1), 82-83.

Howard, J. (1995). You can't get there from here: The need for a new logic in education reform. Proceedings of the American Academic of Arts and Sciences, 124, 85-92.

Hunt, M. M. (1985). Profiles of social research: The scientific study of human interactions. New York: Russell Sage Foundation.

Jones, B. D., Bryant, L. H., Synder, J. D., \& Malone, D. (2012). Preservice and inservice teachers' implicit theories of intelligence. Teacher Education Quarterly, 39(2), 87-101.

Kagan, D. (1992). Professional growth among preservice and beginning teachers. Review of Educational Research, 62, 129-169.

Kaplan, R. G. (1991). Teacher beliefs and practices: A square peg in a square hole. Proceedings of the Thirteenth Annual Meeting of the North American Chapter of the International Group for the Psychology of Mathematics Education, Volume 2 (pp. 119-125). Blacksburg, VA.

Kennedy, M. M. (1999). The role of preservice teacher education. In L. DarlingHammond and G. Sykes (Eds.), Teaching as the learning profession: Handbook of teaching and policy (pp. 54-86). San Francisco, CA: Jossey-Bass.

Kerry, T., \& Kerry, C.A. (1997). Differentiation: Teachers' views of the usefulness of recommended strategies in helping the more able pupils in primary and secondary classrooms. Educational Studies, 23, 439-457. 
Koehler, V. (1985). Research on preservice teacher education. Journal of Teacher Education, 36, 23-30.

Lacey, C. (1977). The socialization of teachers. London: Methuen.

Lave, J. (1988). Cognition in practice. Cambridge: Cambridge University Press.

Lave, J., \& Wenger, E. (1991). Situated learning: Legitimate peripheral participation. Cambridge: Cambridge University Press.

LeCompte, M. D., \& Goetz, J. P. (1982). Problems of reliability and validity in ethnographic research. Review of Educational Research, 52(1), 31-60.

Leroy, N., Bressoux, P., Sarrazin, P., \& Trouilloud, D. (2007). Impact of teachers' implicit theories and perceived pressures on the establishment of an autonomy supportive classroom. European Journal of Psychology of Education, 22(4), 529545.

Lincoln, Y. S., \& Guba, E. G. (1985). Naturalistic inquiry. Newbury Park, CA: Sage. Liston, D., Whitcomb, J., \& Borko, H. Too little or too much: Teacher preparation and the first years of teaching. Journal of Teacher Education, 57(4), 351-358.

Lortie, D. C. (1975). Schoolteacher: A Sociological Study. Chicago, IL: University of Chicago Press.

Lou, Y., Abrami, P. C., Spence, J. C., Poulsen, C., Chambers, B., \& d'Appollonia, S. (1996). Within-class grouping: A meta-analysis. Review of Educational Research, $66,423-458$.

Marbach-Ad, G., \& McGinnis, J. R. (2009). Beginning mathematics teachers' beliefs of subject matter and instructional actions documented over time. School Science and Mathematics, 109(6), 338-354. 
Marks, R. (1990). Pedagogical content knowledge: From a mathematical case to a modified conception. Journal of Teacher Education, 41(3), 3-11.

Marshall, C., \& Rossman, G. B. (2011). Designing qualitative research (5 ${ }^{\text {th }}$ ed.). Thousand Oaks, CA: Sage.

Marulanda, M., Giraldo, P., \& Lopez, L. (2006). Differentiated instruction for bilingual learners. Presentation at Annual Conference of the Association for Supervision and Curriculum Development, San Francisco, CA.

Maxwell, J. A. (1996). Qualitative research design: An interactive approach. Thousand Oaks, CA: Sage.

McCray, E. D., \& McHatton, P. A. (2011). "Less afraid to have them in my classroom": Understanding pre-service general educators' preceptions about inclusion. Teacher Education Quarterly, 38(4), 135-155.

McDiarmid, G. W., Ball, D. L., \& Anderson, C. (1989). Why staying ahead one chapter just won’t work: Subject-specific pedagogy. In M. C. Reynolds (Ed.), Knowledge base for the beginning teacher (pp. 193-205). New York, N.Y.: Pergamon Press.

McDiarmid, G. W., \& Wilson, S. M. (1991). An exploration of the subject matter knowledge of alternate route teachers: Can we assume they know their subject? Journal of Teacher Education, 42, 93-103.

McDonald, F. (1980). The problems of beginning teachers: A crisis in training. Study of Induction Programs for Beginning Teachers, Vol. 1. Princeton, NJ: ETS.

McDonnough, J. T., \& Matkins, J. J. (2010). The role of field experience in elementary preservice teachers' self-efficacy and ability to connect research to practice. Social Science and Mathematics, 110(1), 13-23. 
McGinnis, J. R., Parker, C., \& Graeber, A. O. (2004). A cultural perspective of the induction of five reform-minded beginning mathematics and science teachers. Journal of Research in Science Teaching, 41(7), 720-747.

Merriam, S. B. (1988). Case study research in education: A qualitative approach. San Francisco, CA: Jossey-Bass.

Merriam, S. B. (1998). Qualitative research and case study applications in education. San Francisco, CA: Jossey-Bass.

Miles, M. B., \& Huberman, A. M. (1994). Qualitative data analysis: An expanded sourcebook (2nd ed). Thousand Oaks, CA: Sage.

Monahan, T., \& Fisher, J. A. (2010). Benefits of “observer effects": Lessons from the field. Qualitative Research, 10(3), 357-376.

Monk, D. H. (1994). Subject area preparation of secondary mathematics and science teachers and student achievement. Economics of Education Review, 13, 125-145.

Moon, T. R., Brighton, C. M., \& Callahan, C. M. (2003). State standardized testing programs: Friend or foe of gifted education? Roeper Review, 25, 49-60.

Moon, T. R., Brighton, C. M., Jarvis, J. M., \& Hall, C. J. (2007). State standardized testing programs: Their effects on teachers and students. RM07228. Storrs, CT: The National Research Center on the Gifted and Talented.

Moon, T. R., Tomlinson, C. A., \& Callahan, C. M. (1995). Academic diversity in the middle school: Results of a survey of middle school administrators and teachers. RM95124. Storrs, CT: The National Research Center on the Gifted and Talented. National Association for the Education of Young Children. (2003). Early childhood curriculum, assessment, and program evaluation: Building an effective, 
accountable system in programs for children, birth through age 8. Washington, D.C.

National Association for Teachers of English and The International Reading Association. (2000). Standards for the English language arts. Urbana, IL.

National Council of Teachers of Mathematics. (2006). Curricular focal points for prekindergarten through grade 8 mathematics: A quest for coherence. Reston, VA.

Nespor, J. (1987). The role of beliefs in the practice of teaching. Journal of Curriculum Studies, 19, 317-328.

Nolen, S. B., Ward, C. J., Horn, I. S., Childers, S., Campbell, S. S., \& Mahna, K. (2009). Motivation development in novice teachers: The development of utility filters. In M. Wosnitza, S. A. Karabenick, A. Efllides, \& P. Nenniger (Eds.), Contemporary motivation research: From global to local perspectives (pp. 265-278). Ashland, OH: Hogrefe \& Huber.

Nolen, S. B., Horn, I. S., Ward, C. J., \& Childers, S. A. (2011). Novice teacher learning and motivation across contexts: Assessment tools as boundary objects. Cognition and instruction, 29(1), 88-122.

Oakes, J. (2005). Keeping track: How schools structure inequality ( $2^{\text {nd }}$ ed.). New Haven: Yale University Press.

Oakes, J., Wells, A., Jones, M., \& Datnow, A. (1997). Detracking: The social construction of ability, cultural politics, and resistance to reform. Teachers College Record, 98(3), 482-510. 
Oakhurst (Pseudonym) County Public Schools. (2015). Schools directory website. Retrieved February 22, 2015.

Parajes, M. F. (1992). Teachers' beliefs and educational research: Cleaning up a messy construct. Review of Educational Research, 62, 207-332.

Penuel, W. R., Fishman, B. J., Yamaguchi, R., \& Gallagher, L. P. (2007). What makes professional development effective? Strategies that foster curriculum implementation. American Educational Research Journal 44(4), 921-958.

Penuel, W. R., Sun, M., Frank, K. A., \& Gallagher, H. A. (2012). Using social network analysis to study how collegial interactions can augment teacher learning from external professional development. American Journal of Education, 119, 103-136.

Peressini, D., Borko, H., Romagnano, L., Knuth, E., \& Willis, C. (2004). A conceptual framework for learning to teach secondary mathematics: A situative perspective. Educational Studies in Mathematics, 56, 67-96.

Perry, W. G. (1970). Forms of intellectual and ethical development in the college years. San Francisco, CA: Jossey-Bass.

Prescott, A., \& Cavanagh, M. (2008). A situated perspective on learning to teach secondary mathematics. In M. Goos, R. Brown, \& K. Makar (Eds.), Navigating currents and charting directions: Proceedings of the $31^{\text {st }}$ annual conference of the Mathematics Education Research Group of Australia (pp. 407-413). Brisbane, QLD: MERGA.

Putnam, R. T., \& Borko, H. (2000). What do new views of knowledge and thinking have to say about research on teacher learning? Educational Researcher, 29(1), 4-15. 
Raymond, A. M. (1997). Inconsistency between a beginning elementary school teacher's mathematics beliefs and teaching practice. Journal for Research in Mathematics Education, 28(5), 550-576.

Reis, S. M., McCoach, D. B., Little, C. A., Muller, L. M., \& Kaniskan, R. B. (2011). The effects of differentiated instruction and enrichment pedagogy on reading achievement in five elementary schools. American Educational Research Journal, 48(2), 462-501.

Resnick, L. B. (1988). Treating mathematics as an ill-structured discipline. In R. I. Charles \& E. A. Silver (Eds.), Research agenda for mathematics education: Vol. 3. The teaching and assessing of mathematical problem solving (pp. 32-60). Hillsdale, NJ: Erlbaum.

Rex, L. A., \& Nelson, M. C. (2004). How teachers' professional identities position highstakes test preparation in their classrooms. Teachers College Record, 106(6), 1288-1331.

Richardson, V. (1996). The role of attitudes and beliefs in learning to teach. In J. Silkuda (Ed.), Handbook on research on teacher education (pp. 102-110). New York, NY: MacMillan.

Rowan, B., Chiang, F. S., \& Miller, R. J. (1997). Using research on employees' performance to study the effects of teachers on students' achievements. Sociology of Education, 70, 256-284.

Sands, D. I., \& Barker, H. B. (2004). Organized chaos: Modeling differentiated instruction for preservice teachers. Teaching \& Learning, 19(1), 26-49. 
Santangelo, T., \& Tomlinson, C. A. (2012). Teacher educators' perceptions and use of differentiated instruction practices: An exploratory investigation. Action in Teacher Education, 34, 309-327.

Schoenfeld, A. H. (1998). Toward a theory of teaching-in-context. Issues in Education: Contributions from Cognitive Psychology, 4(1), 1-94.

Schumm, J., Vaughn, S., Haager, D., McDowell, J., Rothlein, L., \& Saumell, L. (1995). General education teacher planning: What can students with learning disabilities expect? Exceptional Children, 61, 335-352.

Schwandt, T. (1994). Constructivist, interpretivist persuasions for human inquiry. In N. Denzin \& Y. Lincoln (Eds.), Handbook of qualitative research (pp. 118-137). Thousand Oaks, CA: Sage.

Schwandt, T. A. (2000). Three epistemological stances for qualitative inquiry: Interpretivism, hermeneutics, and social constructionism. In N. K. Denzin \& Y. S. Lincoln (Eds.), Handbook of qualitative research (2nd ed., pp. 189-214). Thousand Oaks, CA: Sage.

Sherman, S. C. (2009). Haven't we seen this before? Sustaining a vision in teacher education for progressive teaching practice. Teacher Education Quarterly, 36(4), 41-60.

Shulman, L. S. (1986). Those who understand: Knowledge growth in teaching. Educational Researcher, 15(2), 4-14.

Shulman, L. S. (1987). Knowledge and teaching: Foundations of the new reform. Harvard Educational Review, 57, 1-22. 
Siegel, J., \& Shaughnessy, M. F. (1994). Educating for understanding: An interview with Howard Gardner. Phi Delta Kappan, 75(7), 563-66.

Simmons, P. E., Emory, A., Carter, T., Coker, T., Finnegan, B., Crockett, D., ... Labuda, K. (1999). Beginning teachers: Beliefs and classroom actions. Journal of Research in Science Teaching, 36, 930-953.

Simon, M. (1993). Prospective elementary teachers' knowledge of division. Journal for Research in Mathematics Education, 24, 232-254.

Smith, L. M. (1978). An evolving logic of participant observation, educational ethnography and other case studies. In L. Shulman (Ed.), Review of research in education (pp. 316-377). Itasca, IL: Peacock.

Southerland, S., \& Gess-Newsome, J. (1999). Preservice teachers' views of inclusive science teaching as shaped by images of teaching, learning, and knowledge. Science Education, 83(2), 131-150.

Sprinthall, N., \& Thies-Sprinthall, L. (1983). The teacher as an adult learner. In G. Griffin (Ed.), Staff development: The eighty-second yearbook of NSSE (Part II). Chicago, IL: University of Chicago Press.

Stake, R. E. (1995). The art of case study research. Thousand Oaks, CA: Sage.

Stake, R. E. (2006). Multiple case study analysis. New York, NY: Guilford.

Steele, D. F. (2001). The interfacing of preservice and inservice experiences of reformbased teaching: A longitudinal study. Journal of Mathematics Teacher Education, 4, 139-172. 
Steele, D. F., \& Widman, T. F. (1997). Practitioner's research: A study in changing preservice teachers' conceptions about mathematics and mathematics teaching and learning. School Science and Mathematics, 97(4), 184-191.

Sternberg, R.J. (1985). Beyond IQ: A triarchic theory of human intelligence. New York: Cambridge University Press.

Stoddart, T., Connell, M., Stofflett, R., \& Peck, M. (1993). Reconstructing elementary teacher candidates' understanding of mathematics and science content. Teaching and Teacher Education, 9, 229-241.

Stofflett, R., \& Stoddart, T. (1992). Patterns of assimilation and accommodation in traditional and conceptual change teacher education courses. Paper presented at the Annual Meeting of the American Educational Research Association, San Francisco.

Stodolsky, S. S., \& Grossman, P. (2000). Changing students, changing teaching. Teachers College Record, 102, 125-172.

Struyven, K., Dochy, F., \& Janssens, S. (2008). The effects of hands-on experience in students' preferences for assessment methods. Journal of Teacher Education, 59, 69-88.

Tabachnick, B., \& Zeichner, K. (1984). The impact of student teaching experience on the development of teacher perspectives. Journal of Teacher Education, 35(6), 28-36.

Thompson, J., Windschitl, M., \& Braaten, M. (2013). Developing a theory of ambitious early-career teacher practice. American Educational Research Journal. 50(3), 574-615. 
Tieso, C. (2002). The effects of grouping and curricular practices on intermediate students' math achievement. Hartford, CT: National Research Center on the Gifted and Talented, University of Connecticut.

Tom, A. R. (1997). Redesigning teacher education. Albany, NY: State University of New York Press.

Tomlinson, C.A. (1999). The differentiated classroom: Responding to the needs of all learners. Alexandria, VA: ASCD.

Tomlinson, C.A. (2003). Fulfilling the promise of the differentiated classroom: Tools and strategies for responsive teaching. Alexandria, VA: Association for Supervision and Curriculum Development.

Tomlinson, C.A. (2005). How to differentiate instruction in mixed-ability classrooms $\left(2^{\text {nd }}\right.$ ed.). Alexandria, VA: ASCD.

Tomlinson, C. A. (2014). The differentiated classroom ( $2^{\text {nd }}$ ed.). Alexandria, VA: ASCD.

Tomlinson, C., Brighton, C., Hertberg, H., Callahan, C., Moon, T., Brimijoin, K., Conover, L., \& Reynolds, T. (2003). Differentiating instruction in response to student readiness, interest, and learning profile in academically diverse classrooms: A review of literature. Journal for the Education of the Gifted, 27, 119-145.

Tomlinson, C. A., Brimijoin, K., \& Narvaez, L. (2008). The differentiated school: Making revolutionary changes to teaching and learning. Alexandria, VA: ASCD. Tomlinson, C. A., Callahan, C. M., Moon, T. R., Tomchin, E. M., Landrum, M., Imbeau, M., ... Eiss, N. (1995). Preservice teacher preparation in meeting the needs of 
gifted and other academically diverse students. RM 95134. Storrs, CT: The National Research Center on the Gifted and Talented.

Tomlinson, C. A., Callahan, C. M., Tomchin, E. M., Eiss, N., Imbeau, M., \& Landrum, M. (1997). Becoming architects of communities of learning: Addressing academic diversity in contemporary classrooms. Exceptional Children, 63(2), 269-282.

Tomlinson, C. A., \& Imbeau, M. B. (2010). Leading and managing a differentiated classroom. Alexandra, VA: ASCD.

Tomlinson, C. A. \& Javius, E. L. (2012). Teach up for excellence. Educational Leadership, 69(5), 28-33.

Tomlinson, C. A., \& McTighe, J. (2006). Integrating differentiated instruction and understanding by design: Connecting content and kids. Alexandria, VA: Association for Supervision and Curriculum Development.

Tomlinson, C. A., \& Moon, T. R. (2013). Assessment and student success in a differentiated classroom. Alexandria, VA: Association for Supervision and Curriculum Development.

Tomlinson, C. A., Tomchin, E. M., Callahan, C. M., Adams, C. M., Pizzat-Tinnin, P., Cunningham, C. M., ... Imbeau, M. (1994). Practices of preservice teachers related to gifted and other academically diverse learners. Gifted Child Quarterly, 38(3), 106-114.

Tricario, K., \& Yendol-Hoppey, D. (2012). Teacher learning through self-regulation: An exploratory study of alternatively prepared teachers' ability to plan differentiated instruction in an urban elementary school. Teacher Education Quarterly, 39(1), 139-158. 
U.S. Congress. (2002). No Child Left Behind Act of 2001. Public Law 107-110, 107 ${ }^{\text {th }}$

Congress. Washington, D.C.: Government Printing Office.

U.S. Department of Education. (2002). The Secretary's report on teacher quality.

Washington, D.C.: U.S. Department of Education.

Utley, B. L. (2006). Effects of situated learning on knowledge gain of instructional strategies by students in a graduate level course. Teacher Education and Special Education, 29, 69-82.

Valli, L., \& Agostinelli, A. (1993). Teaching before and after professional preparation: The story of a high school mathematics teacher. Journal of Teacher Education, 44, 107-118.

Veenman, S. (1984). Perceived problems of beginning teachers. Review of Educational Research, 54(2), 143-178.

Wasserman, S., \& Faust, K. (1994). Social network analysis: Methods and application. New York, NY: Cambridge University Press.

Watanabe, M. (2006). "Some people think this school is tracked and some people don't:" Using inquiry groups to unpack teachers' perspectives on detracking. Theory into Practice, 45(1) 24-31.

Weinstein, C. S. (1990). Prospective elementary teachers' beliefs about teaching: Implications for teacher education. Teaching and Teacher Education, 6(3), 279290.

Wenger, E. (1998). Communities of practice. New York: Cambridge University Press. 
Wertheim, C., \& Leyser, Y. (2002). Efficacy beliefs, background variables, and differentiated instruction of Israeli prospective teachers. The Journal of Educational Research, 96(1), 54-63.

Whitaker, S. D. (2001). Supporting beginning special education teachers. Focus on Exceptional Children, 34, 1-18.

Wideen, M., Mayer-Smith, J., \& Moon, B. (1998). A critical analysis of the research on learning to teach: Making the case for an ecological perspective on inquiry. Review of Educational Research, 68(2), 130-178.

Wiggins, G. \& McTighe, J. (2005). Understanding by design (2nd ed.). Alexandria, VA: ASCD.

Wilson, M. (1994). One preservice secondary teacher's understanding of function: The impact of a course integrating mathematical content and pedagogy. Journal for Research in Mathematics Education, 25, 346-370.

Wilson, S. M., Floden, R. E., \& Ferrini-Mundy, J. (2002). Teacher preparation research: An insider's view from the outside. Journal of Teacher Education, 53(3), 190204.

Wilson, S. M., Shulman, L. S., \& Richert, A. E. (1987). “150 different ways” of knowing: Representations of knowledge in teaching. In J. Calderhead (Ed.), Exploring teachers' thinking (pp. 104-124). London: Cassell.

Wilson, S. M., \& Wineburg, S.S. (1988). Peering at history through different lenses. Teachers College Record, 89, 525-539. 
Woods, P. (1992). Symbolic interaction: Theory and method. In M. D. LeCompte, W. L. Millroy, \& J. Preissle (Eds.), The handbook of qualitative research in education (pp. 337-404). New York, NY: Academic Press W2.

Yin, R. K. (2009). Case study research: Design and methods $\left(4^{\text {th }}\right.$ ed.). Los Angeles, CA: Sage.

Yin, R. K., \& Davis, D. (2007). Adding new dimensions to case study evaluations: The case of evaluating comprehensive reforms. In G. Julnes \& D. J. Rog (Eds.), Informing federal policies for evaluation methodology (New Directions in Program Evaluation, No. 113, pp. 75-93). San Francisco, CA: Jossey-Bass.

Youngs, P. (2007). District induction policy and new teachers' experiences: An examination of local policy implementation in Connecticut. Teachers College Record, 109, 797-837.

Youngs, P., Frank, K. A., \& Pogodzinski, B. (2012). The role of mentors and colleagues in beginning elementary and middle school teachers' language arts instruction. In S. Kelly (Ed.), Understanding teacher effects (pp. 161-181). New York, NY: Teachers College Press.

Zeichner, K. M. (1980). Myths \& realities: Field-based experiences in preservice teacher education. Journal of Teacher Education, 31, 45-47.

Zeichner, K. M., \& Gore, J. M. (1990). Teacher socialization. In W. R. Houston (Ed.), Handbook of research on teacher education (pp. 329-547). New York: Macmillian. 
Zeichner, K. M., \& Tabachnick, R. T. (1981). Are the effects of university teacher education 'washed out' by school experience? Journal of Teacher Education, 23(3), 7-11.

Zeichner, K. M., \& Tabachnick, R. T. (1985). The development of teacher perspectives: Social strategies and institutional control in the socialization of beginning teachers. Journal of Education for Teaching, 11(1), 1-25.

Zeichner, K. M., Tabachnick, R. T., \& Densmore, K. (1987). Individual, institutional, and cultural influences on the development of teachers' craft knowledge. In J. Calderhead (Ed.), Exploring teachers' thinking (pp. 21-59). London: Cassell. 
Appendix A

Dates of Differentiating Instruction Course Meetings and Topics Discussed Spring 2014

\begin{tabular}{|c|c|}
\hline DATE & TOPICS DISCUSSED \\
\hline $\begin{array}{c}\text { Week } 1 \\
\text { January } 14 \\
\end{array}$ & $\begin{array}{l}\text { - Introduction to the course } \\
\text { - } \quad \text { Differentiation's audience (who we teach) }\end{array}$ \\
\hline $\begin{array}{c}\text { Week } 2 \\
\text { January } 21\end{array}$ & $\begin{array}{ll}\text { - } & \text { Defining differentiation } \\
\text { - } & \text { Learning environment (where we teach) }\end{array}$ \\
\hline $\begin{array}{c}\text { Week } 3 \\
\text { January } 28\end{array}$ & $\begin{array}{l}\text { - Learning environment (where we teach) (cont.) } \\
\text { ○ Role of mindset in differentiation } \\
\text { - Low-preparation differentiation strategies }\end{array}$ \\
\hline $\begin{array}{c}\text { Week } 4 \\
\text { February } 4\end{array}$ & $\begin{array}{l}\text { - Effective curriculum as differentiation's starting point (what we } \\
\text { teach) } \\
\quad \text { Teaching up and respectful tasks } \\
\text { - Low-preparation differentiation strategies (cont.) }\end{array}$ \\
\hline $\begin{array}{c}\text { Week } 5 \\
\text { February } 11\end{array}$ & $\begin{array}{l}\text { - Effective curriculum as differentiation's starting point (what we } \\
\text { teach) (cont.) } \\
\text { o Writing KUDs }\end{array}$ \\
\hline $\begin{array}{l}\text { Week } 6 \\
\text { February } 18\end{array}$ & $\begin{array}{l}\text { - Effective curriculum as differentiation's starting point (what we } \\
\text { teach) (cont.) } \\
\text { - Modification of instruction by readiness }\end{array}$ \\
\hline $\begin{array}{c}\text { Week } 7 \\
\text { February } 25\end{array}$ & - Modification of instruction by readiness (cont.) \\
\hline $\begin{array}{r}\text { Week } 8 \\
\text { March } 4\end{array}$ & $\begin{array}{ll}\text { - } & \text { Modification of instruction by learning profile } \\
\text { - } & \text { Ongoing assessment }\end{array}$ \\
\hline $\begin{array}{l}\text { Week } 9 \\
\text { March } 11\end{array}$ & Spring Recess - NO CLASS \\
\hline $\begin{array}{l}\text { Week 10 } \\
\text { March } 18\end{array}$ & - Modification of instruction by learning profile (cont.) \\
\hline $\begin{array}{l}\text { Week } 11 \\
\text { March } 25\end{array}$ & $\begin{array}{ll}\text { - } & \text { Modification of instruction } \\
\text { - } & \text { Strategies for modifying instruction: RAFTs, learning contracts }\end{array}$ \\
\hline $\begin{array}{l}\text { Week } 12 \\
\text { April } 1\end{array}$ & $\begin{array}{ll}\text { - } & \text { Modification of instruction by interest } \\
\text { - } & \text { Ongoing assessment (cont.) }\end{array}$ \\
\hline $\begin{array}{c}\text { Week } 13 \\
\text { April } 8\end{array}$ & - Leading and managing a differentiated classroom \\
\hline $\begin{array}{l}\text { Week 14 } \\
\text { April } 15\end{array}$ & - Leading and managing a differentiated classroom \\
\hline $\begin{array}{l}\text { Week } 15 \\
\text { April } 22\end{array}$ & - Grading in a differentiated classroom \\
\hline
\end{tabular}


Appendix B

Differentiating Instruction Course Reflection Prompts

Spring 2014

\section{REFLECTION 1 - Due Week 2 (January 19, 2014)}

Choose 2 or 3 of the students who were introduced in class this week. Imagine yourself in the role of the teacher of a class which includes those students. (If the students presented were a different age than the age group you would teach, it's fine to consider who they were in the past or who they might be in the future for this post.)

It's the beginning of the school year. Write a reflection as their teacher about (a) what you want to happen for those 2 or 3 students this year in terms of their growth and (b) what you will do to create a learning environment that's supportive and encouraging in light of what they bring to your classroom.

\section{REFLECTION 2 - Due Week 4 (February 2, 2014)}

In class on Tuesday, we talked about the roles that mindset, connections, and community play in establishing a supportive learning environment.

- Consider your own K-12 educational experiences.

- Describe a time or two when one or more of these three elements of a supportive learning environment was not present and you think it should have been.

- What should have happened in that classroom that didn't in terms of beliefs and practices?

- Consider what your own classroom would be like if you aspired to establish a supportive learning environment through these three elements. Michael suggested that 1 out of 8 classrooms feels like an inviting place to learn, while Aaron thought the number was more like $2 \%$. Most teachers would probably say that they want their classrooms to feel welcoming and supportive, but it seems in many cases that they're missing the mark.

- Why do you think this is?

- How would some of your beliefs or practices reflecting mindset, connections, and/or community stretch you as an educator? What might the challenges be for you, and how would you overcome them?

\section{REFLECTION 3 - Due Week 6 (February 16, 2014)}

At this point of the semester, you've already completed one-third of the course. Consider how your ideas about the course content have developed over the past five weeks, and answer the following questions in a Microsoft Word document:

- Consider the "ah ha" or "hang on a sec" moments you've experienced so far in the course through class discussions, readings, workshops, and your own reflection. 
- Out of all the new ideas you've encountered, which one resonated the most with your own beliefs or teaching practices? Why?

- What's been the most surprising thing you've learned about differentiation? Why was it such a surprise?

- Out of all the new ideas you've encountered, which one created the most dissonance, challenge, confusion, or conflict in you? Why?

- Consider the class discussions, readings, and workshop on high quality curriculum we've done in the last few weeks.

- Out of the elements of planning for engagement, focusing on understanding, teaching up, and writing clear KUDs, which one or two would present the greatest challenge for you in your own teaching practice? Why? What might you do to overcome that challenge?

- (If you choose writing clear KUDs, make sure your response goes beyond saying: I'm not good at writing them yet/I need more practice!)

- What's the biggest question you still have about the principles or practices of differentiation (other than wanting to learn more concrete strategies and see more specific examples of differentiation in action)?

\section{REFLECTION 4 - Due Week 11 (March 23, 2014)}

We are now two-thirds of the way through the course. We've talked about learning environment, quality curriculum, modifying instruction by readiness and learning profile, and a bit about assessment. While we've looked at some specific strategies which support these elements of a differentiated classroom, we've also considered larger principles and philosophical issues which underlie differentiation.

As you've thought about differentiation and these specific elements, what connections have you made to learning and learners?

1. If you've taught, student taught, or observed in a field placement, what specific connections have you made between your experiences with individual kids or groups of kids in those settings and the ideas you've considered in this course? Are there aspects of a differentiated classroom which would have been especially beneficial for the learners you have in mind? Are there any aspects of a differentiated classroom, perhaps those which are causing dissonance or confusion for you, which you don't see as directly connecting to those learners?

If you haven't taught, student taught, or observed in a field placement, consider the same questions as they apply to kids you've worked with in other contexts.

(Regardless of your teaching experience, if you'd prefer to answer question 1 about a sibling, other family member, or another learner you know very well from a different context, that's fine.)

2. Consider yourself as a learner and educational experiences you've had which were not in differentiated classrooms. Are there aspects of a differentiated classroom which would 
have been especially beneficial for you? Was there a particular time in your education when you think it would have benefited you the most? Are there any aspects of a differentiated classroom, perhaps those which are causing dissonance or confusion for you, which you don't see immediately connecting to yourself as a learner?

3. What's the one question you still have about differentiation which you hope to have answered by the end of this course? Why is it such an important question for you to figure out the answer to? (Consider questions which might feasibly be answered by the end of the course, rather than "How will I have time during my first year of teaching to do everything I want to?")

\section{REFLECTION 5 - Due Week 13 (April 6, 2014)}

Review the KUDs for the course listed on pages 2-3 of the syllabus. [These KUDs appear below at the end of this Appendix.]

1. Us:

- For which U has your own depth of understanding developed the most this semester? (Please type out the full $\mathrm{U}$ in your response.)

- Describe your growth in understanding that $\mathrm{U}$.

- How would you describe your thinking about the ideas in that U before the course began?

- How did your ideas change over time?

- Was there a particular turning point or "aha!" moment at which you experienced significant growth in this $\mathrm{U}$, or was it so gradual you can't point to a particular moment? Why do you think that is?

- Which whole class or small group discussions, individual reflections, readings, or assignments contributed the most to your growth in this $\mathrm{U}$ ?

- How will your understanding of this $U$ contribute to your future practice as an educator?

- For which U do you feel you still have the most growth to do in deepening your understanding? (Please type out the full $U$ in your response.)

- How did your understanding of this U develop throughout the course (even though it hasn't developed quite as deeply as you'd like yet)?

- What is it about this $U$ that causes it to be the one you still need to grow in the most?

- What will you do in your future practice as an educator to support your continued growth in this $\mathrm{U}$ ?

2. Ks: Think about how you would have defined these Ks on the first day of the course and how you would define them now. Choose the K for which you think your definition has changed the most. 
- Describe how you would have defined it on the first day (or explain that you wouldn't have known enough to give a definition at all), and give your current definition in your own words.

- Describe your growth in coming to know or better appreciate this K:

- Was there a particular turning point or "aha!" moment at which your knowledge of this term expanded significantly, or was it so gradual you can't point to a particular moment? Why do you think that is?

- Which whole class or small group discussions, individual reflections, readings, or assignments contributed the most to your growth with this $\mathrm{K}$ ?

- Explain how your knowledge of this term will play a role in your future practice as an educator.

3. Ds:

- For which D has your proficiency developed the most this semester? (Please type out the full $\mathrm{D}$ in your response.)

- Describe your growth in being able to do the skill.

- How would you describe your proficiency with the skill before the course began?

- How did your proficiency change over time in the course?

- Was there a particular turning point or "aha!" moment at which you experienced significant growth in this skill, or was it so gradual you can't point to a particular moment? Why do you think that is?

- Which whole class or small group discussions, individual reflections, readings, or assignments contributed the most to your growth in this skill?

- How will your proficiency with this skill contribute to your future practice as an educator?

\section{DIFFERENTIATING INSTRUCTION COURSE KUD LEARNING OBJECTIVES}

At the end of this course, students should:

\section{UNDERSTAND THAT}

- Differentiation is a philosophy of responsive teaching designed to maximize the capacity of each learner.

- Differentiation professionalizes teachers and is respectful of students, individually and as a group.

- Defensible differentiation reflects five non-negotiable, interdependent elements which address environment, curriculum, assessment, instruction, and management.

- Creating an environment that actively supports students in the work of learning through a growth mindset, connections among the teacher and students, and a strong classroom community encourages intellectual risk-taking and growth.

- Absolute clarity about a powerful learning destination and teaching up to help students reach that destination promote student engagement and understanding.

- Continuously knowing where students are in relation to the learning destination through ongoing assessment informs instruction and provides students with meaningful feedback on their performance. 
- Modifying instruction in response to student readiness, interest, and learning profile helps ensure that each student arrives at the learning destination, and moves beyond it when possible.

- Effective leadership and management allow different students to work on different tasks at different times efficiently and independently within a flexible classroom.

\section{KNOW}

- key terminology of differentiation, including:

o mindset

$\bigcirc$ on-going assessment (pre-, formative, summative)

- flexible grouping

- respectful tasks

- readiness, interest, learning profile

$\circ$ teaching up

- Know-Understand-Do (KUD) learning goals.

- key instructional strategies for differentiation.

\section{BE ABLE TO}

- Develop clear KUDs.

- Align KUDs, assessments, and learning activities.

- Design lessons using key instructional strategies which modify content, process, or products based on student readiness, interest, or learning profile.

- Analyze and evaluate differentiated tasks using the non-negotiables and key terminology. Reflect on plans for leading and managing a differentiated classroom.

- Reflect on personal philosophy and practice in relation to the non-negotiables and key terminology.

- Synthesize critical ideas from selected resources on differentiation.

- Create a product for an external audience which extends understanding and insight regarding the principles and practices of differentiation. 


\author{
Appendix C \\ Phase 1 Round 1 Interview Questions \\ January 17-30, 2014 \\ (all 20 participants)
}

Teaching Aspirations \& Screening

- Tell me about your personal philosophy of teaching and learning.

○ Where do you think that philosophy came from?

- Has your philosophy changed while you've been at Curry? If so:

- How did it change?

- Tell me about why you think it changed.

- Do you hope to teach in the fall? If so:

- At what level are you hoping to teach?

○ Where are you looking at jobs?

- Tell me about your ideal teaching situation.

- What would the school setting be like?

- What grade(s) would you teach? Why?

$\circ$ What subjects would you teach? Why?

$\circ$ When you think about all the different kinds of learners you might encounter in a classroom, what kinds of learners would you teach in your ideal setting?

- What kinds of learners wouldn't be there?

Conceptions of Differentiation

- How would you explain differentiation to someone who had never heard of it?

- Tell me about a concrete example of what differentiation looks like in action.

- Tell me about a concrete example of how you might use differentiation in your future classroom.

- Do you have any hesitancies/misgivings about differentiating in a classroom? If so:

o Tell me about them.

- What knowledge, understanding, and skills do you want to learn from the course?

External Factors: Other Teacher Ed Experiences (Coursework and Field Placements)

- Where do you think your current understanding of differentiation came from?

$\circ$ Did you hear about it in any of your other courses?

- Which ones?

- What did you hear?

○ Did you learn anything about differentiation from your field placements?

- Which ones?

- What did you learn?

- Have you read anything about differentiation before?

- Where?

- What did you learn?

○ Did you learn about it from any other experiences?

- Tell me about those experiences.

- What did you learn? 


\section{Appendix D}

Phase 1 Round 2 Interview Questions

February 24-26, 2014

(11 focal participants only)

Conceptions of Differentiation In class, we've been using Adam's definition as a framework for thinking about differentiation. We've talked about Adam's definition having five parts: learning environment, curriculum, assessment, the modification of instruction, and leading and managing the classroom. So far in the course, we've talked about three of those components. We've finished talking about learning environment and curriculum, and we've started talking about modifying instruction-specifically about modifications based on student readiness.

- Learning environment

- How would you explain the idea of a classroom learning environment?

- Has the way you think about a classroom learning environment changed in this course, or has it stayed the same as before the course? If it's changed:

- Tell me about how it's changed.

- In class, we've talked about how the five components of a differentiated classroom are interdependent as they work together in a system. How would you explain the role of the learning environment in that system?

- High quality curriculum

- How would you explain the idea of high quality curriculum?

- Has the way you think about high quality curriculum changed in this course, or has it stayed the same as before the course? If it's changed:

- Tell me about how it's changed.

- If you think again about how the five components of a differentiated classroom work together in a system, how would you explain the role of high quality curriculum in that system?

- Modification of instruction based on readiness

- How would you explain the idea of student readiness?

- How would you explain the idea of modifying instruction based on readiness?

- Has the way you think about responding to student readiness changed in this course, or has it stayed the same as before the course? If it's changed:

- Tell me about how it's changed.

External Factors: Other Teacher Ed Experiences (Field Placements)

Background info

- 588 Placement [Repeat questions if participant had two 588 placements.]

$\circ$ When did you do your 588 placement?

- Which school were you in?

O Which grades and subjects did you teach?

- 488 Placements

-When did you do your 488 placements?

- Which schools were you in?

○ Which grades and subjects did you teach? 
- 388 Placements

○ When did you do your 388 placements?

- Which schools were you in?

- Which grades and subjects did you teach?

\section{Field placement learning experiences}

- Tell me about your 588 placement. [Repeat questions if participant had two 588 placements.]

○ How would you describe the kind of teacher your CI was?

- How would you describe the atmosphere of the school?

o How would you describe the atmosphere of the classroom?

- What was your role in the classroom?

○ Overall, how would you characterize the experience you had in 588?

$\circ$ Do you think your experiences in this placement influenced your thinking about differentiation? If so:

- How do you think they influenced your thinking about differentiation?

○ Did you and your university supervisor ever talk about differentiation?

- If so, what did you talk about?

- Tell me about your 488 placements.

- What was your role in the classroom for the first placement?

- What was your role in the classroom for the second placement?

○ Overall, how would you characterize the experience you had in your first 488 placement?

- How would you characterize the experience you had in your second 488 placement?

○ Do you think your experiences in these placements influenced your thinking about differentiation? If so:

- How did they influence your thinking about differentiation?

- Tell me about your 388 placements.

- What was your role in the classroom for the first placement?

- What was your role in the classroom for the second placement?

- Overall, how would you characterize the experience you had in your first 388 placement?

○ How would you characterize the experience you had in your second 388 placement?

○ Do you think your experiences in these placements influenced your thinking about differentiation? If so:

- How did they influence your thinking about differentiation?

- Have you had experiences in any other classrooms which may have influenced your thinking about differentiation?

o What were they?

○ How did they influence your thinking about differentiation? 


\author{
Appendix E \\ Phase 1 Round 3 Interview Questions \\ April 9-10, 2014 \\ (11 focal participants only)
}

External Factors: Personal Background Factors (Prior Experiences)

- Before you first got to Curry, what did you think good teaching in an elementary/ secondary classroom looked like?

- Has your answer changed since then?

- If so: Tell me about how it's changed.

- If not: Tell me why you think it hasn't changed.

- Some people have suggested that a teacher's personality or other personal characteristics influence how they teach.

- How do you think your personal traits have influenced how you teach?

- Do you think how those traits influence your teaching might change over time with more experience?

- If so, how might it change?

- Think back to your own experiences in elementary and secondary school.

○ Do you think any of your teachers differentiated instruction? If so:

- What did differentiation look like in their classrooms?

- How did it work for you as a learner?

- How do you think it worked for other learners in your classes?

- In classrooms where your teachers' instruction wasn't differentiated, how would you describe their instruction?

- How did that instruction work for you as a learner?

- How do you think it worked for other learners in that class?

- When people are working to understand a philosophy like differentiation, they sometimes feel there are certain personal experiences they've had in the past that influence their understanding. For example, I've heard people say that, as they learned more about differentiation, they kept thinking about who their younger brother was as a learner and how he would have responded to a differentiated classroom as a kid, or something that their grandmother used to say that became their family motto, or an experience they had as a learner with a particular teacher. Is there an experience you've had that you think may have influenced your think about differentiation? If so: Tell me about it.

Conceptions of Differentiation

In class, we've continued using Adam's definition as a framework for thinking about differentiation. We've talked about Adam's definition having five parts involving learning environment, curriculum, assessment, the modification of instruction, and leading and managing the classroom.

At this point in the course, we've talked about all five of those components. When we did our last interview, we'd finished talking about learning environment and curriculum, and we'd started talking about modifying instruction based on readiness. Since then, we finished talking about modifying instruction based on readiness, and then we talked about modifying instruction based on interest, modifying instruction based on 
learning profile, ongoing assessment, and leading and managing a differentiated classroom.

- Modification of instruction based on readiness

- If another teacher told you that he wanted to try modifying instruction based on readiness for the first time and wanted your advice on how to do it effectively, what would you say?

- Do you see yourself trying any of the readiness strategies we talked about in class when you're in the classroom? (If not, tell me about why you think that is.) If so:

- Which ones do you see yourself trying?

- Tell me about why you're planning to use those.

- Modification of instruction based on interest

- Has the way you think about student interest changed in this course, or has it stayed the same as before the course?

- If it's changed: Tell me about how it's changed.

- Modification of instruction based on learning profile

- How would you explain the idea of student learning profile?

- Has the way you think about student learning profile changed in this course, or has it stayed the same as before the course?

- If it's changed: Tell me about how it's changed.

- Ongoing assessment

- Has the way you think about assessment changed in this course, or has it stayed the same as before the course?

- If it's changed: Tell me about how it's changed.

- In class, we've talked about how the five components of a differentiated classroom are interdependent as they work together in a system. How would you explain the relationship between assessment and curriculum within that system?

- How would you explain the relationship between assessment and instruction within that system?

- Leading and managing a differentiated classroom

- In this class, we've talked about leading a classroom and managing a classroom as different ideas.

- How would you explain the idea of leading a differentiated classroom?

- How would you explain the idea of managing a differentiated classroom?

- Has the way you think about leading and managing a classroom changed in this course, or has it stayed the same as before the course?

- If it's changed: Tell me about how it's changed.

- If you think again about how the five components of a differentiated classroom work together in a system, how would you explain the role of leading and managing the classroom within that system? 


\author{
Appendix F \\ Phase 1 Round 4 Interview Questions \\ May 1-9, 2014 \\ (all 20 participants)
}

Conceptions of Differentiation

- During our first interview, I asked you about your personal philosophy of teaching and learning. Has that philosophy changed at all this semester, or has it stayed the same?

If it's changed, how did it change?

$\circ$ Were there particular moments when you realized those changes had occurred?

- How would you explain differentiation to someone who had never heard of it?

$\circ$ I asked you the same question during our first interview in January. How do you think your answer today is different from your answer then?

- What was your biggest misconception about differentiation at the course's start?

o When did you realize you had this misconception?

$\circ$ What's your new understanding of the issue?

- Tell me about a concrete example of what differentiation looks like in action.

○ I asked you the same question during our first interview in January. How do you think your answer today is different from your answer then?

Concept Mapping Activity These five cards show the five components of differentiation according to Adam's definition that we've been using all semester. Please use those cards and this piece of paper to draw a concept map that illustrates the relationship among these five components in a differentiated classroom.

- Tell me about your concept map.

- I only gave you cards with these five components. Is there anything else that needs to go on this map beyond the concepts on the cards?

- Sometimes when people draw a concept map like this to demonstrate relationships among ideas, they feel like the relationships between some of the ideas are stronger than the relationships between other ideas. Are some of the relationships between the components you've shown here stronger than others, or are they all the same strength?

- If I'd asked you to make this concept map on the first day of the course, how might you have done it?

Differentiation and Future Practice

- When you enter the classroom, how might differentiation be a part of your thinking and your practice?

- Some teachers build responding to anticipated student needs into their planning, and some respond more after the needs have arisen. Do you think differentiation will be a part of your planning, or do you see it happening more after some needs have arisen in your classroom?

- Tell me about why you think that is. 
- Do you think differentiation will be part of your thinking and practice in your first year of teaching?

- If so: How do you think it will be a part of your thinking and practice?

- If not: Tell me about why you think that is.

- Do you have any hesitancies about trying differentiation? If so:

○ Tell me about what they are.

- What have you learned about differentiation this semester that you don't feel clear enough on to move forward with in your own classroom?

- What else do I need to know about your understanding of differentiation at this point? 


\section{Appendix G}

Phase 2 Rounds 1-3 Interview Questions

September, October, \& November 2014

(5 focal participants)

Observation Follow-Up

- Tell me about the lesson I just saw.

- Tell me about the unit this lesson is a part of.

- How did you decide to use (the instructional strategy/assessment) in today's lesson?

- How did you group students for today's lesson?

- How did assessment play a role in today's lesson?

- Was differentiation on your mind when you planned the lesson?

If so, how?

O If not, why do you think it didn't play a role?

- Was differentiation on your mind when you taught the lesson?

$\circ$ If so, how?

If not, why do you think it didn't play a role?

Conceptions of Differentiation

- How would you explain the idea of differentiation at this point?

- Has the way you think about differentiation changed this fall?

- If so,

- How did it change?

- Was there a specific experience you had that's related to the change?

- Are there particular students in your class who're related to the change?

- If not, why did you think it hasn't changed?

- Have you gained any new knowledge about differentiation since we last spoke?

- Have your beliefs about differentiation changed this fall? (e.g., importance, feasibility)

- Have some particular principles of differentiation been on your mind since we last spoke?

If so, which ones? How have you been thinking about them?

- Which of the five components of a differentiated classroom have been at the forefront of your thinking recently?

○ In the coming weeks, do you think which components are at the forefront of your thinking will change?

- If so, how?

- If not, why do you think that is? 
Concept Mapping Activity ( $2^{\text {nd }}$ interview only)

In May, I asked you to take these five cards showing the elements of a differentiated classroom and create a concept map that that illustrated the relationships among them. I'm going to ask you to do that again for me now so that I can see your thinking about how a differentiated classroom works now that you're a first year teacher. Please think out loud as you consider how to design your map and then create it.

- Tell me about your concept map.

- I only gave you cards with these five components. Is there anything else that needs to go on this map in addition to the concepts on the cards?

- Sometimes when people draw a concept map like this to demonstrate relationships among ideas, they feel like the relationships between some of the ideas are stronger than the relationships between other ideas. Are some of the relationships between the components you've shown here stronger than others, or are they all the same strength?

Here's the concept map you created in May.

- How is your new map similar to the map you created in May?

- How would you explain the reason for those similarities?

- How is it different?

- How would you explain the reason for those differences?

Relationship between Conceptions and Practice

- Tell me about the learning environment in your classroom.

o How would you describe it?

- What did you to do to establish it at the beginning of the year?

- What role did your students have in establishing it?

- Tell me about your curriculum.

- How much of your curriculum are you creating, and how much of it is given to you?

○ How much freedom do you have in designing your curriculum?

- What resources are you using to design your curriculum?

- Who else is involved in your curriculum design process?

- Tell me about assessment in your classroom.

○ What kinds of assessments are you using in your classroom?

- What are you learning from your assessments?

- What's the relationship between your assessment and your instruction?

- Tell me about your classroom management.

- How has it compared with what you expected as a preservice teacher?

- How would you describe your style of classroom management?

- Tell me about how you've responded to different student needs in your teaching.

- Tell me about your students' needs.

- How would you describe your students' readiness with what you're teaching right now? Have differences in their readiness been a factor in your teaching so far?

- Has student interest or learning profile played a role in your teaching so far? 
- How aware are your students of the differences among their learning needs?

- Have there been any breakthroughs/obstacles in your teaching practice since the last time we talked? Tell me about them.

Contextual Factors

Mindset

- Tell me about your mindset.

- Have there been any changes in your mindset this fall compared to the spring?

- Do you see a relationship between your mindset and your teaching?

- Do you see a relationship between your students' mindset and their work?

Differentiating Instruction Course

- Which principle or practice you learned about in the class has been the most useful?

○ How has it been useful?

- Which assignment from the course was the most useful?

○ How was it useful?

- Which topic do you wish you'd learned more about now that you're in the classroom?

Culture of Practice

- How would you describe your school's/department's culture?

- How does your administration/mentor teacher/department chair play a role in your teaching practice?

- Where, when, how often do you interact with them?

o How aware are they of what and how you're teaching?

○ Have they observed you? Have they given you feedback on your teaching?

- What's the relationship between them and your growth in your teaching practice?

- How do your fellow teachers play a role in your teaching practice?

$\circ \quad$ Who do you interact with regularly?

- When, where, how often do you interact with them?

o What do you usually talk about?

- How aware are they of what you're doing in your teaching practice? How aware are you of what they're doing in their teaching practice?

$\circ$ Have you observed them teach?

○ Have they observed you teach? Have they given you feedback on your teaching?

- Do you hear differentiation talked about in your building?

$\circ$ If so, how is the term being used?

$\circ$ When, where, how often is it used?

- Do you feel differentiation is being practice by other teachers?

- How would you describe their knowledge of differentiation?

○ How would you describe their beliefs about differentiation or its principles?

- Has your faculty had any professional development on differentiation in the past? 
- Will your faculty have any professional development on it this year?

- What factors inside your classroom support your implementation of differentiation?

- What factors inside your classroom challenge your implementation of differentiation?

- What factors outside your classroom (i.e., department, school, district, national, social factors) support your implementation?

- What factors outside your classroom challenge your implementation? 


\author{
Appendix $\mathrm{H}$ \\ Phase 2 Round 4 Interview Questions \\ December 2014 \\ Karen
}

This week, I read through all of the transcripts of the interviews we did in January through May and all of the assignments you wrote for the Differentiating Instruction course. I looked for any factor you mentioned that might have been related to your conceptions of differentiation while you were taking the course. I'm using the word conceptions to encompass both your knowledge of differentiation, such as your knowledge of its principles and specific practices, and your beliefs about it, such as how important it is. I made a list of all those factors.

When I looked over the list, I noticed that the factors seemed to fall into six categories: (a) beliefs about teaching and learning you had before the course, (b) your experiences as a K-12 student, (c) your personal characteristics such as your personality traits, $(d)$ your experiences in the differentiation course, $(e)$ your experiences in the other courses you took as a preservice teacher, and $(f)$ your experiences in your field placements. [I showed a list of these six categories as I named them.] Do you have any questions about what those six categories mean?

I'm going to show you a list of all the factors I identified in which I've paraphrased your comments. You'll see that I've given each factor (or pair of factors if two went together) a header that's one of the six categories I just mentioned. You'll also see that I've included that the date on which you talked about that factor so that you can see at what point during the semester you mentioned it.

I'm going to ask you to classify each factor (or pair of factors if two went together) in one of four ways: (a) This factor enhanced my conceptions of differentiation. (b) This factor detracted from my conceptions of differentiation. (c) This factor wasn't related to my conceptions of differentiation. (d) I'm not sure whether this factor was related to my conceptions of differentiation. Remember, I'm defining conceptions to mean your knowledge and your beliefs about differentiation. So I'm asking you to think about whether each factor enhanced your knowledge or beliefs about differentiation or detracted from your knowledge or beliefs about differentiation.

Here's the list of factors I'd like you to consider. As you go down the list and classify each factor, please tell me why you're classifying it that way. [Participants had the list of four classifications options in front of them as they reviewed the factors.] Do you have any questions about what I'm asking you to do? If you read a factor and you're not sure what it means, ask me. 


\begin{tabular}{|c|}
\hline How is this factor related to your conceptions of differentiation? \\
\hline $\begin{array}{l}\text { PRIOR BELIEFS ABOUT TEACHING } 1 \\
*_{\text {must put students first in teaching } 1 / 27} \\
*_{\text {must make learning meaningful (through enjoyment, personal connections, relevance) }} \\
1 / 27\end{array}$ \\
\hline $\begin{array}{l}\text { PERSONAL CHARACTERISTICS } 1 \\
* \text { gentle, warm, fun, playful personality } 4 / 10 \\
* \text { caring - eager to connect with students as individuals } 4 / 10\end{array}$ \\
\hline $\begin{array}{l}\text { PERSONAL CHARACTERISTICS } 2 \\
*_{\text {saw father as a kind of teacher who instilled values in daughters in different ways based }} \\
\text { on age, experience, and personality } 4 / 10\end{array}$ \\
\hline $\begin{array}{l}\text { PERSONAL CHARACTERISTICS } 3 \\
\text { *"I like things very structured and very routine, and I think sometimes I do need to be } \\
\text { pushed a little bit out of my comfort zone" to give students more independence - not to } \\
\text { assume they're going to have trouble with some things like routines. } 4 / 10\end{array}$ \\
\hline $\begin{array}{l}\text { PERSONAL CHARACTERISTICS } 4 \\
\text { *has growth mindset } 5 / 01 \\
\text { *sees all students as capable } 5 / 01\end{array}$ \\
\hline $\begin{array}{l}\text { K-12 EXPERIENCES } 1 \\
* 5 \text { th grade gifted class }- \text { strong learning environment, effective instruction } 2 / 26,4 / 10\end{array}$ \\
\hline $\begin{array}{l}\text { K-12 EXPERIENCES } 2 \\
\text { *didn't experience differentiation, instead experienced one-size-fits all instruction } 4 / 10 \\
* \text { worked fine for her as a student because good at memorizing things and studied hard but } \\
\text { did not work well for peers who struggled to learn } 4 / 10\end{array}$ \\
\hline $\begin{array}{l}\text { FIELD EXPERIENCE } 1 \\
\text { *student teaching CI was "progressive," emphasized student choice and comfort, } \\
\text { differentiated by interest (exp. Wed. research project) and learning profile } 1 / 27,2 / 26, \\
4 / 10\end{array}$ \\
\hline $\begin{array}{l}\text { FIELD EXPERIENCE } 2 \\
\text { *student teaching CI wasn't always warm with kids; was sometimes blunt, harsh, stern } \\
2 / 26\end{array}$ \\
\hline $\begin{array}{l}\text { FIELD EXPERIENCE } 3 \\
\text { *seeing more examples of differentiation in field experiences would have been helpful } \\
5 / 01\end{array}$ \\
\hline $\begin{array}{l}\text { OTHER CURRY COURSES } 1 \text { - amount of discussion of DI in other courses } \\
*_{\text {* }} \text { only touched on DI very briefly in general methods, read one article on it } 1 / 27 \\
*_{\text {very minimal discussion of DI } 5 / 01}\end{array}$ \\
\hline 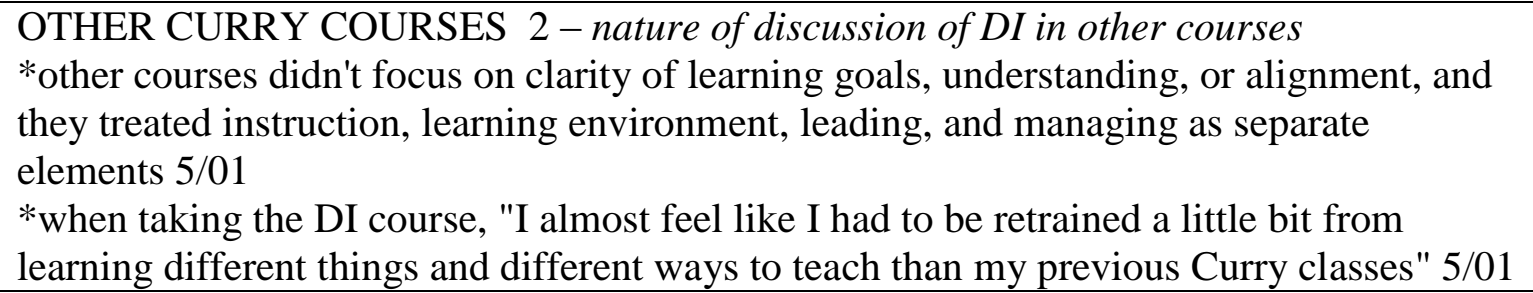 \\
\hline
\end{tabular}




\section{COURSE 1}

*developed more complete understanding of components of modification of instruction $5 / 01$

*came to recognize DI as an all-encompassing philosophy $5 / 01$

*used shopping cart metaphor reflecting interconnectedness of classroom elements 4/10

DI COURSE 2 - fact that it was taken during final semester of coursework

*"Part of me wishes I would have had this before my student teaching or my first semester here, but I'm kind of glad I had it at the end because it think it just ties it all together." 5/01

OTHER 1? Was there anything else that enhanced your conceptions of differentiation?

OTHER 2? Was there anything else that detracted from your conceptions of differentiation?

Now please look back at the factors on the list that you said enhanced your conceptions of differentiation and the factors you said detracted from your conceptions. Out of all those factors that influenced your conceptions positively or negatively, what are the top three that had the strongest relationship to your conceptions? Please rank them for me 1, 2, 3, with 1 being the factor with the strongest relationship to your conceptions, and tell me why that relationship is so strong.

This week, I also read through all of the transcripts of the interviews we did this fall, and I looked for any factor you mentioned in relation to differentiation that might have been connected to your teaching practice this fall. I made a list of all those factors.

When I looked over the list, I noticed that the factors seemed to fall into three categories: (a) the nature of the culture of practice at Newland, (b) your position as Newland's kindergarten teacher, and (c) who your students are, such as their past experiences and personal traits. [I showed a list of these three categories as I named them.] Do you have any questions about what those three categories mean?

I'm going to show you a list of all the factors I identified in which I've paraphrased your comments. You'll see that once again I've given each factor (or pair of factors if two went together) a header that's one of the three categories I just mentioned. You'll also see that I've again included that the date on which you talked about that factor so that you can see at what point during the fall you mentioned it.

I'm going to ask you to classify each factor (or pair of factors if two went together) in one of four ways: (a) This factor supported my implementation of differentiation. (b) This factor challenged my implementation of differentiation. (c) This factor wasn't related to my implementation of differentiation. (d) I'm not sure whether this factor was related to my implementation of differentiation. So I'm asking you to think about whether each factor played a role in your teaching practice related to differentiation.

Here's the list of factors I'd like you to consider. As you go down the list and classify each factor, please tell me why you're classifying it that way. [Participants had the list of four classifications options in front of them as they reviewed the factors.] Do you have any questions about what I'm asking you to do? If you read a factor and you're not sure what it means, ask me. 


\begin{tabular}{|c|}
\hline How is this factor related to your implementation of differentiation? \\
\hline $\begin{array}{l}\text { CULTURE OF PRACTICE } 1 \\
*_{\text {school puts philosophy of differentiation into practice } 9 / 01} \\
\text { *DI comes up in almost every conversation with teachers } 11 / 17 \\
\text { *administration responded to individual teacher interests and learning profiles in } \\
\text { orientation - reflecting philosophy } 9 / 01 \\
*_{\text {school expects meaningful, purposeful, challenging curriculum } 9 / 01}\end{array}$ \\
\hline 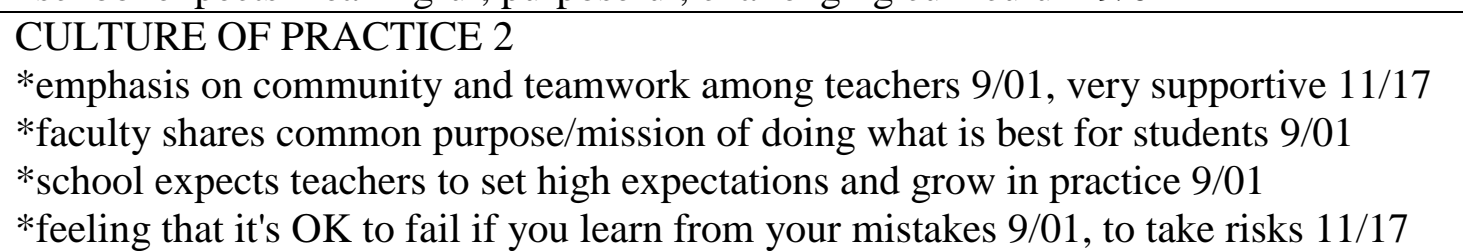 \\
\hline $\begin{array}{l}\text { NATURE OF JOB } 1 \\
* \text { only kindergarten teacher } 9 / 29\end{array}$ \\
\hline $\begin{array}{l}\text { NATURE OF JOB } 2 \\
* \text { "so much freedom" in curriculum and instruction } 9 / 29, \text { not prescribed } 11 / 17 \\
*_{\text {not required to use certain textbook series } 9 / 29}\end{array}$ \\
\hline $\begin{array}{l}\text { NATURE OF JOB } 3 \\
* \text { lack of available technology } 9 / 29\end{array}$ \\
\hline $\begin{array}{l}\text { NATURE OF JOB } 4 \\
* \text { "hasn't been as much planning and collaboration time as I had hoped" with other } \\
\text { teachers on faculty } 9 / 29, \text { wishes that interacting with other teachers was a bit more } \\
\text { helpful } 11 / 17 \\
\text { *has mentor teacher, initially anticipated meeting regularly but didn't end up doing so } \\
11 / 17 \\
\text { *administration and other teachers aren't aware of what her instruction looks like - no } \\
\text { observations } 11 / 17\end{array}$ \\
\hline $\begin{array}{l}\text { NATURE OF JOB } 5 \\
\text { *room is spacious, groups can spread out } 11 / 17\end{array}$ \\
\hline $\begin{array}{l}\text { NATURE OF JOB } 6 \\
* \text { has assistant } 11 / 17\end{array}$ \\
\hline $\begin{array}{l}\text { NATURE OF JOB } 7 \\
* \text { flexible timing - can alter daily schedule a bit when needed } 11 / 17\end{array}$ \\
\hline $\begin{array}{l}\text { WHO STUDENTS ARE } 1 \\
\text { *major readiness differences among students } \\
\text { * }_{\text {some not recognizing all letters yet, one may be reading on } 3 \text { rd grade level } 9 / 01,9 / 29} \\
11 / 17 \\
\text { *in math, some students have number concept like end of } 1 \text { st grade, others have very } \\
\text { limited number sense } 11 / 17\end{array}$ \\
\hline $\begin{array}{l}\text { WHO STUDENTS ARE } 2 \\
* \text { all students went to preschool 9/29, 11/17 }\end{array}$ \\
\hline $\begin{array}{l}\text { WHO STUDENTS ARE } 3 \\
\text { *students aren't reading yet, can't be handed a set of directions for an activity to work } \\
\text { independently } 9 / 29\end{array}$ \\
\hline
\end{tabular}


WHO STUDENTS ARE 4

*students uphold behavioral expectations when working in groups and transitioning $11 / 17$

PERSONAL CHARACTERISTICS 5

*has growth mindset, especially with steep learning curve $11 / 17$

*willing to ask for support from a variety of resources outside school 11/17

PARTICIPATION IN THIS RESEARCH STUDY

OTHER 1? Was there anything else that supported implementation?

OTHER 2? Was there anything else that challenged implementation?

[Karen added that having a teaching assistant who was not a certified teacher was a factor that challenged implementation.]

Now please look back at the factors on the list that you said supported your implementation of differentiation and the factors you said challenged your implementation. Out of all those factors that influenced your implementation positively or negatively, what are the top three that had the strongest relationship to your implementation? Please rank them for me 1, 2, 3, with 1 being the factor with the strongest relationship to your implementation, and tell me why that relationship is so strong. 


\author{
Appendix I \\ Phase 2 Round 4 Interview Questions \\ December 2014 \\ Nicole
}

This week, I read through all of the transcripts of the interviews we did in January through May and all of the assignments you wrote for the Differentiating Instruction course. I looked for any factor you mentioned that might have been related to your conceptions of differentiation while you were taking the course. I'm using the word conceptions to encompass both your knowledge of differentiation, such as your knowledge of its principles and specific practices, and your beliefs about it, such as how important it is. I made a list of all those factors.

When I looked over the list, I noticed that the factors seemed to fall into six categories: (a) beliefs about teaching and learning you had before the course, (b) your experiences as a K-12 student, (c) your personal characteristics such as your personality traits, $(d)$ your experiences in the differentiation course, $(e)$ your experiences in the other courses you took as a preservice teacher, and $(f)$ your experiences in your field placements. [I showed a list of these six categories as I named them.] Do you have any questions about what those six categories mean?

I'm going to show you a list of all the factors I identified in which I've paraphrased your comments. You'll see that I've given each factor (or pair of factors if two went together) a header that's one of the six categories I just mentioned. You'll also see that I've included that the date on which you talked about that factor so that you can see at what point during the semester you mentioned it.

I'm going to ask you to classify each factor (or pair of factors if two went together) in one of four ways: (a) This factor enhanced my conceptions of differentiation. (b) This factor detracted from my conceptions of differentiation. (c) This factor wasn't related to my conceptions of differentiation. (d) I'm not sure whether this factor was related to my conceptions of differentiation. Remember, I'm defining conceptions to mean your knowledge and your beliefs about differentiation. So I'm asking you to think about whether each factor enhanced your knowledge or beliefs about differentiation or detracted from your knowledge or beliefs about differentiation.

Here's the list of factors I'd like you to consider. As you go down the list and classify each factor, please tell me why you're classifying it that way. [Participants had the list of four classifications options in front of them as they reviewed the factors.] Do you have any questions about what I'm asking you to do? If you read a factor and you're not sure what it means, ask me. 


\begin{tabular}{|c|}
\hline How is this factor related to your conceptions of differentiation? \\
\hline $\begin{array}{l}\text { PRIOR BELIEFS ABOUT TEACHING } 1 \\
\text { * believes that every student is capable of learning, no student is unreachable if they } \\
\text { want to be reached } 1 / 27 \\
\text { *a successful day in her classroom is when everybody goes home feeling respected, } \\
\text { important, and safe, and wants to come back the next day } 4 / 10\end{array}$ \\
\hline $\begin{array}{l}\text { PERSONAL CHARACTERISTICS } 1 \\
\text { *Doesn't have a fully growth mindset, but wants to. "It's difficult to undo } 18 \text { years of } \\
\text { schooling that reinforced all day every day" a fixed mindset } 2 / 24\end{array}$ \\
\hline $\begin{array}{l}\text { PERSONAL CHARACTERISTICS } 2 \\
\text { *"I'm a person who really likes planning and lists and to be very, very organized....You } \\
\text { can't modify instruction until you see what's in front of you. I couldn't in August plan } \\
\text { my differentiation ideas for the first few units because I won't even have met my } \\
\text { students yet. It's a little bit like mental dissonance when my personality wants it to be } \\
\text { this one way, and you have to accept that it's not always going to be that way." } 2 / 24\end{array}$ \\
\hline $\begin{array}{l}\text { PERSONAL CHARACTERISTICS } 3 \\
\text { *"not super patient," has most patience with a student who is trying really hard and just } \\
\text { not succeeding, loses patience the most when a student is not trying } 4 / 10\end{array}$ \\
\hline $\begin{array}{l}\text { K-12 EXPERIENCES } 1 \\
\text { *"I am totally a product of fixed mindset classrooms." } 2 / 24 \\
\text { *"I see tracking as the ultimate manifestation of a fixed mindset" } 2 / 24 \\
\text { *believes the system encourages some kids to keep going in advanced classes to } \\
\text { continue to succeed, and believes other students won't succeed } \\
\text { *classmates saw each other as a competitors and not learning partners } 2 / 24\end{array}$ \\
\hline $\begin{array}{l}\text { K-12 EXPERIENCES } 2 \\
\text { *identified as gifted. "A big reason I was successful in school is because at a very } \\
\text { young age, enough people told me that I was smart...I definitely believed in school that } \\
\text { there were smart kids and there were dumb kids. I was in the class with the smart kids" } \\
2 / 24\end{array}$ \\
\hline $\begin{array}{l}\text { K-12 EXPERIENCES } 3 \\
\text { *generally her teachers did not differentiate } 4 / 10 \\
\text { *6th grade math teacher was "really good at using a lot of different access points to } \\
\text { information," had clear learning goals, taught conceptually } 4 / 10\end{array}$ \\
\hline $\begin{array}{l}\text { K-12 EXPERIENCES } 4 \\
\text { *learning experiences that were based on readiness didn't always work well for less } \\
\text { advanced peers. Example: fourth grade basals at different levels, competition for who } \\
\text { could get through books the fastest, awareness of who was low readiness (in purple } \\
\text { books) } 4 / 10\end{array}$ \\
\hline $\begin{array}{l}\text { OTHER CURRY COURSES } 1 \\
\text { *introduction to C\&I course - read Tomlinson text. But at time, wasn't clear on how DI } \\
\text { would work in classroom, thought it meant individualized instruction } 1 / 27 \\
\text { *DI "woven" into other Curry classes but discussed in "vague" terms } 1 / 27\end{array}$ \\
\hline
\end{tabular}


FIELD EXPERIENCE 1

*student teaching CI differentiated word study by readiness $1 / 27$

*student teaching CI was good at establishing community and routines 2/24 FIELD EXPERIENCE 2

*saw different interpretation of DI in Belfast, didn't like how it was done 1/27

FIELD EXPERIENCE 3

*one teacher in 488 modeled effective teaching practice $2 / 24$

FIELD EXPERIENCE 4

*student teaching classroom had really high ELL population 4/10

DI COURSE 1

*came to see differentiation as a philosophy rather than a set of strategies or instruction

$2 / 24$

*"folded" ideas about differentiation into existing beliefs about teaching 2/24

*DI became "lens" through which she viewed instruction, causing "all these other pieces to fall into place" $2 / 24$

*understanding of tiering changed during course to reflect teaching up and need for all students to have same KUDs 5/06

DI COURSE 2

*had frequent conversations with other elementary and elementary special ed students to "talk stuff out and tease out ideas and make sense of something" 5/06

OTHER 1? Was there anything else that enhanced your conceptions of differentiation?

OTHER 2? Was there anything else that detracted from your conceptions of differentiation?

Now please look back at the factors on the list that you said enhanced your conceptions of differentiation and the factors you said detracted from your conceptions. Out of all those factors that influenced your conceptions positively or negatively, what are the top three that had the strongest relationship to your conceptions? Please rank them for me 1, 2, 3, with 1 being the factor with the strongest relationship to your conceptions, and tell me why that relationship is so strong.

This week, I also read through all of the transcripts of the interviews we did this fall, and I looked for any factor you mentioned in relation to differentiation that might have been connected to your teaching practice this fall. I made a list of all those factors.

When I looked over the list, I noticed that the factors seemed to fall into three categories: (a) the nature of the culture of practice at Newland, (b) your position as Newland's kindergarten teacher, and (c) who your students are, such as their past experiences and personal traits. [I showed a list of these three categories as I named them.] Do you have any questions about what those three categories mean?

I'm going to show you a list of all the factors I identified in which I've paraphrased your comments. You'll see that once again I've given each factor (or pair of factors if two went together) a header that's one of the three categories I just mentioned. You'll also see that I've again included that the date on which you talked about that factor so that you can see at what point during the fall you mentioned it. 
I'm going to ask you to classify each factor (or pair of factors if two went together) in one of four ways: (a) This factor supported my implementation of differentiation. (b) This factor challenged my implementation of differentiation. (c) This factor wasn't related to my implementation of differentiation. (d) I'm not sure whether this factor was related to my implementation of differentiation. So I'm asking you to think about whether each factor played a role in your teaching practice related to differentiation.

Here's the list of factors I'd like you to consider. As you go down the list and classify each factor, please tell me why you're classifying it that way. [Participants had the list of four classifications options in front of them as they reviewed the factors.] Do you have any questions about what I'm asking you to do? If you read a factor and you're not sure what it means, ask me.

\begin{tabular}{|l|}
\hline \multicolumn{1}{|c|}{ How is this factor related to your implementation of differentiation? } \\
\hline CULTURE OF PRACTICE 1 \\
*collaborative, everyone is willing to work with others and to share $8 / 25$ \\
\hline CULTURE OF PRACTICE 2 \\
*new teachers are supported by: mentor, lead mentor, lead teacher/more experienced \\
teachers on team, instructional coaches, \& AP, who are "very approachable" $9 / 30$ \\
*"I've gotten a lot of new teacher support" $11 / 14$ \\
*meets regularly with mentor, mentor has co-taught some lessons and helped assess \\
students in math 9/30 \\
\hline CULTURE OF PRACTICE 3 \\
*curriculum is set by state standards, division pacing guide, and team-developed \\
curriculum map $8 / 25$ \\
*had to start teaching content earlier in year than would have liked due to time pressures \\
9/30 \\
*plans together with teammates for full day when district hires subs $9 / 30$ \\
*"given permission not to follow lock-step" the school-level curriculum map $9 / 30$ \\
*ended 1 st quarter behind in math pacing guide, felt the need to "pick it up" and "move a \\
little faster" through curriculum in 2 nd quarter. $11 / 14$ \\
\hline CULTURE OF PRACTICE 4 \\
*Title 1 school reflects significant diversity in SES, with half of population living in \\
poverty/needy and other half solidly middle class $8 / 25$ \\
*teachers trained in how to deal with students living in poverty, saw connection to \\
differentiation in the emphasis on student choice $8 / 25$ \\
\hline NATURE OF JOB 1 \\
*has ELL resource specialist stationed in classroom $8 / 25$ \\
\hline NATURE OF JOB 2 \\
*3rd grade team is "young," four of them are first year teachers $8 / 25$ \\
\hline NATURE OF JOB 3 \\
*standards-based report card ended up having flexibility to reflect where students are \\
with standards, growth, and effort $11 / 14$ \\
\hline
\end{tabular}




\section{NATURE OF JOB 4}

*half of students spend morning with another teacher in French immersion, don't join her class until mid-way through day 9/30

WHO STUDENTS ARE 1

*Almost all students are not native English speakers, many with low WIDA scores reading well below grade level $8 / 25$

*overall, students are very low academically $9 / 30$

*has most ESOL students of any 3rd grade teacher 9/30

WHO STUDENTS ARE 2

*student population she ended up teaching was different than anticipated student population when first hired by division and assigned to different school 8/25

WHO STUDENTS ARE 3

*classroom of 21 includes 17 Hispanic students, 1 Nepalese student, 1 AfricanAmerican student, and 2 Caucasian students.

FIELD EXPERIENCE

*student teaching school was a lot like school she ended up teaching in (some similarities in terms of lower level readers ) 9/30

PARTICIPATION IN THIS RESEARCH STUDY

OTHER 1? Was there anything else that supported implementation?

OTHER 2? Was there anything else that challenged implementation?

Now please look back at the factors on the list that you said supported your implementation of differentiation and the factors you said challenged your implementation. Out of all those factors that influenced your implementation positively or negatively, what are the top three that had the strongest relationship to your implementation? Please rank them for me 1, 2, 3, with 1 being the factor with the strongest relationship to your implementation, and tell me why that relationship is so strong. 
Appendix J
Phase 2 Interview Questions for Administrator or Mentor Teacher

Fall 2014

Beth (Karen's principal) \& Sharon (Nicole's mentor)

- How long have you worked at the school?

- How long have you been in your current position?

- How would you describe your duties in your current position?

- How would you describe the school's culture?

- How would you describe what the instruction looks like in a typical classroom here?

- Tell me about your role as a first-year teacher's administrator/mentor/department chair/colleague.

- How do you interact with a first-year teacher?

- When do those interactions usually happen?

- In what context?

- How often do they occur?

- How are those interactions structured?

- Are there regular meetings or conversations?

- Do you have a formal or informal agenda for the conversation?

- How much of the conversation is directed by you, and how much of it is directed by the first year teacher?

- What sort of support or advice do you usually give first year teachers?

- Do all first year teachers get the same kind of support, or does it differ from teacher to teacher?

- How might the support they receive change over the course of the first year?

- How might it change during their second year and beyond?

- What other sources of support does a first year teacher have in your department/school/district?

- Are those supports formal and structured or informal and unstructured?

- My study is focused on new teachers' beliefs about differentiated instruction during their first year in the classroom.

- How would you explain the idea of differentiation?

- If you thought about the classroom of a teacher whose work with differentiation was strong, how would you describe what differentiation looked like in that classroom?

- How did you learn about differentiation?

- Does it have much of a role at your school? How so?

- Have most of the faculty members here participated in professional development on differentiated instruction?

- Who provided it?

- What did it involve?

○ What do you think the average teacher in your department/school knows about differentiation? 
- What do you think the average teacher in your department/school believes about differentiation in terms of its importance and feasibility?

- What do you think the role of differentiation should be in a first-year teacher's classroom? 